\title{
Integration of Micro-cantilevers with Photonic Structures for Mechano-optical Wavelength Selective Devices
}

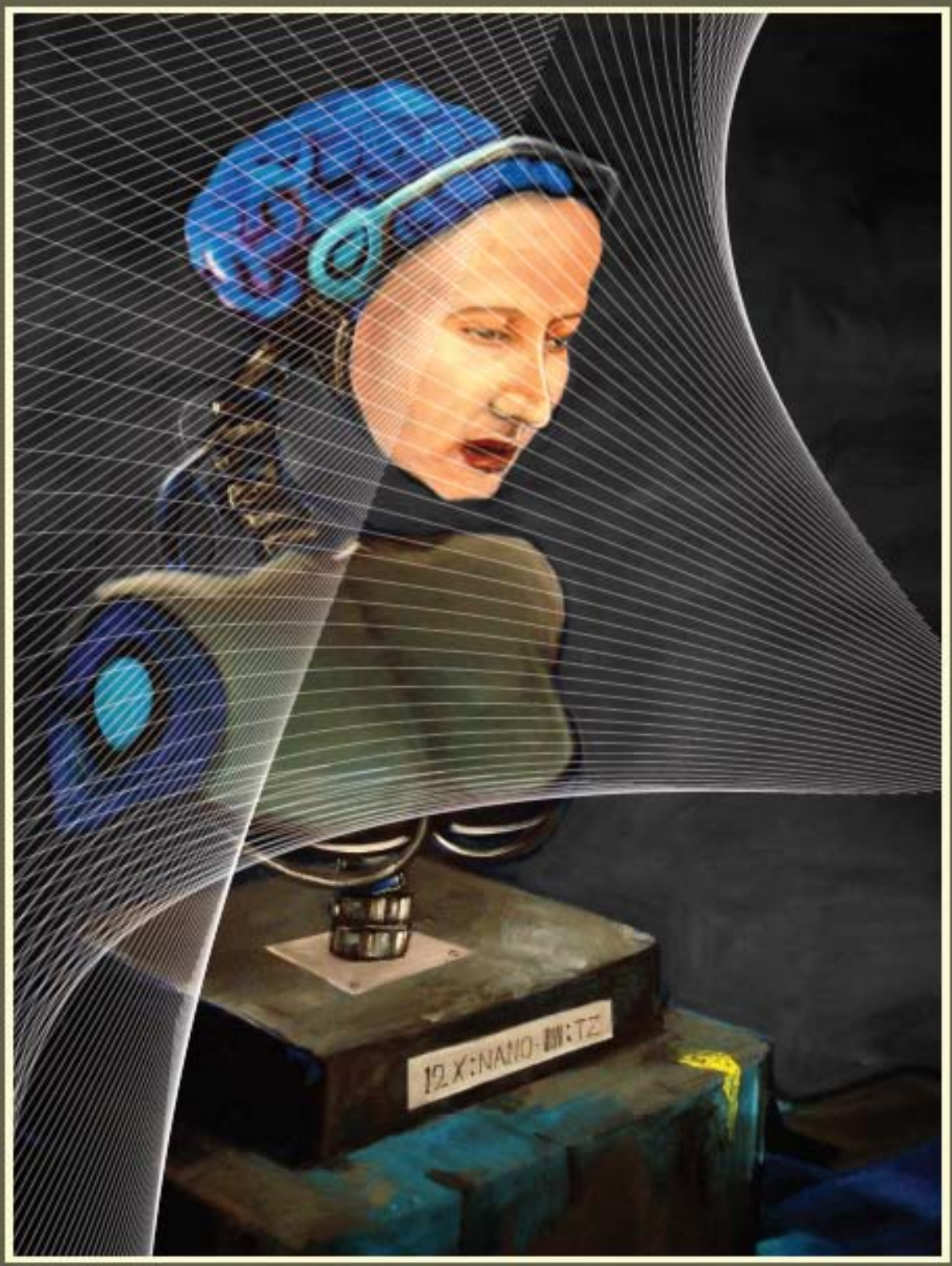

Shahina Mumthaz Chakkalakkal Abdulla 


\section{INTEGRATION OF MICRO- CANTILEVERS WITH PHOTONIC STRUCTURES FOR MECHANO- OPTICAL WAVELENGTH SELECTIVE DEVICES}

Shahina Mumthaz Chakkalakkal Abdulla 


\section{Graduation committee:}

Chairman and secretary:

Prof. dr. ir. A. J. Mouthaan University of Twente

Promotor:

Prof. dr. ir. G. J. M. Krijnen University of Twente

Members:

Prof. dr. J.G.E. Gardeniers University of Twente

Prof. dr. K.J. Boller University of Twente

Prof. dr. H.W.M. Salemink Delft University of Technology

Dr. ir. R.M. de Ridder University of Twente

Dr. ir. H. Tilmans IMEC, Belgium

Dr. M. Hammer University of Twente

\section{nano ned unvessirt of tweerte. MESA+}

The research described in this thesis was carried out at the Transducers

Science and Technology Group of the $\mathrm{MESA}^{+}$research institute, University of Twente, The Netherlands. It has been financially supported by the nanotechnology investment programme, NanoNed of the Dutch ministry of economic affairs, under the project name NEMS: Optical switching by NEMS-actuated resonator arrays" (TOE 7144).

Cover design by Shafi Quraishy (http://www.shafiquraishy.com/)

Printed by Wöhrmann Print Service, Zutphen, The Netherlands

Copyright (C) 2011 by Shahina Mumthaz Chakkalakkal Abdulla, Enschede, The Netherlands. All rights reserved.

DOI: $10.3990 . / 1.9789036531764$

ISBN: 978-90-365-3176-4 


\title{
INTEGRATION OF MICRO- CANTILEVERS WITH PHOTONIC STRUCTURES FOR MECHANO- OPTICAL WAVELENGTH SELECTIVE DEVICES
}

\author{
DISSERTATION
}

to obtain

the degree of doctor at the University of Twente, on the authority of the rector magnificus, prof. dr. H. Brinksma, on account of the decision of the graduation committee, to be publicly defended on Wednesday, 06 April 2011 at 16:45 hrs.

by

Shahina Mumthaz Chakkalakkal Abdulla born on 09 May 1981 in Karupadanna, India 
This dissertation has been approved by:

The promotor: Prof. dr. ir. G. J. M. Krijnen 
...to Aditya

for all the moments

we missed each other. 



\section{Table of Contents}

\section{$1 \quad$ INTRODUCTION AND OUTLINE 1}

$\begin{array}{lll}1.1 & \text { INTRODUCTION }\end{array}$

1.2 OPTICAL DEVICES INVESTIGATED 4

1.2.1 MICRO-RING RESONATOR 4

1.2.2 PHOTONIC CRYSTAL 5

$\begin{array}{lll}1.3 & \text { NEMS-PROJECT } & 6\end{array}$

1.3.1 EPIXFAB: SILICON PHOTONICS FABRICATION PLATFORM

$\begin{array}{ll}1.4 & \text { OUTLINE OF THE THESIS } \\ 1.5 & 8\end{array}$

1.5 REFERENCES 9

$\underline{2} \quad$ MECHANO-OPTICAL MODULATORS

2.1 VARIOUS OPTICAL MODULATION SCHEMES: PREVIOUS WORKS

2.1.1 THERMO-OPTIC EFFECT 12

$\begin{array}{lll}2.1 .2 & 12\end{array}$

$\begin{array}{lll}\text { 2.1.3 PLASMA-DISPERSION EFFECT } & 13\end{array}$

$\begin{array}{ll}2.1 .4 & 13\end{array}$

$\begin{array}{lll}\text { 2.1.5 MECHANO-OPTICAL INTERACTION } & 14\end{array}$

$\begin{array}{lll}2.2 & \text { OUR APPROACH } & \mathbf{2 0}\end{array}$

$\begin{array}{lll}2.3 & \text { CONCLUSIONS } & 21\end{array}$

2.4 REFERENCES

$\underline{3} \quad$ MICRO-BIMORPH CANTILEVERS

$\begin{array}{lll}3.1 & \text { INTRODUCTION } & \mathbf{2 6}\end{array}$

$\begin{array}{lll}3.2 & \text { MODEL } & 28\end{array}$

$\begin{array}{lll}3.2 .1 & \text { STATIC DEFLECTION } & 28\end{array}$

3.2.2 MECHANICAL RESONANCE FREQUENCY 31

$\begin{array}{lll}3.2 .3 & \text { ELECTRO-MECHANICAL COUPLING } & 31\end{array}$

3.3 OPTIMISATION 35

$\begin{array}{lll}3.4 & \text { FABRICATION } & 37\end{array}$

3.5 MEASUREMENTS 39 
$\begin{array}{lll}\text { 3.5.1 RESIDUAL STRESS } & 40\end{array}$

$\begin{array}{lll}3.5 .2 & \text { OFF-STATE DEFLECTION }\end{array}$

$\begin{array}{lll}3.5 .3 & \text { RESONANCE FREQUENCY }\end{array}$

3.5.4 ELECTRO STATIC SPRING SOFTENING EFFECT 42

$\begin{array}{lll}3.6 & \mathbf{4 4}\end{array}$

3.6.1 RESONANCE FREQUENCY 44

3.6.2 PULL-IN VOLTAGE AND ESS EFFECT 46

$\begin{array}{lll}3.7 & \text { DISCUSSIONS } & \mathbf{4 8}\end{array}$

$\begin{array}{lll}3.7 .1 & \text { PULl-IN VOLTAGE }\end{array}$

$\begin{array}{lll}3.7 .2 & \text { OFF-STATE DEFLECTION }\end{array}$

$3.8 \quad$ CONCLUSIONS

3.9 REFERENCES

4 MICRO-CANTILEVER INTEGRATED MICRO-RING RESONATOR:

FABRICATION

$\begin{array}{lll}4.1 & \text { INTRODUCTION } & 56\end{array}$

$\begin{array}{llr}4.2 & \text { BASIC PRINCIPLE } & 57\end{array}$

$\begin{array}{lll}4.3 & \text { FABRICATION } & \mathbf{5 8}\end{array}$

$\begin{array}{lll}\text { 4.3.1 OPTICAL DEVICE FABRICATION } & 58\end{array}$

4.3.2 MICRO-CANTILEVER INTEGRATION

$\begin{array}{lll}4.3 .3 & \text { MATERIALS SELECTION } & 61\end{array}$

4.3.4 MICRO-CANTILEVER GEOMETRY 62

4.3.5 PROTRUSIONS ON THE CANTILEVER 63

4.4 FABRICATION RELATED ISSUES: STRINGER FORMATION AND REMOVAL

$\begin{array}{lll}\text { 4.4.1 STRINGER FORMATION } & 65\end{array}$

$\begin{array}{lll}\text { 4.4.2 STRINGER ELIMINATION METHODS } & 67\end{array}$

4.5 CONCLUSIONS

$\begin{array}{lll}\text { 4.6 REFERENCES } & \mathbf{7 7}\end{array}$

5 MICRO-CANTILEVER INTEGRATED MICRO-RING RESONATOR:

MEASUREMENTS

$\begin{array}{llr}5.1 & \text { INTRODUCTION } & \mathbf{8 0}\end{array}$

5.2 MECHANICAL CHARACTERISATION 8

$\begin{array}{lll}\text { 5.2.1 OFF-STATE DEFLECTION } & 81\end{array}$ 
$\begin{array}{lll}\text { 5.2.2 RESONANCE FREQUENCY } & 82\end{array}$

$\begin{array}{lll}\text { 5.2.3 QUALITY FACTOR } & 85\end{array}$

$\begin{array}{lll}\text { 5.2.4 RESPONSE TIME } & 87\end{array}$

$\begin{array}{lll}\text { 5.2.5 PULL-IN VOLTAGE } & 88\end{array}$

$\begin{array}{lll}5.3 & \text { OPTICAL CHARACTERISATION } & 94\end{array}$

5.3.1 MEASUREMENT SET UP $\quad 94$

$\begin{array}{lll}\text { 5.3.2 OPTICAL LOSSES } & 96\end{array}$

$\begin{array}{lll}\text { 5.3.3 WAVELENGTH TUNING } & 97\end{array}$

$\begin{array}{lll}\text { 5.3.4 DYNAMIC SWITCHING } & 99\end{array}$

$\begin{array}{lll}5.4 & \text { DISCUSSION } & 100\end{array}$

$\begin{array}{lll}5.5 & \text { CONCLUSIONS } & 101\end{array}$

$\begin{array}{lll}5.6 & \text { REFERENCES } & 101\end{array}$

6 PHOTONIC CRYSTAL SLABS WITH

$\begin{array}{ll}\text { INTEGRATED MICRO-CANTILEVERS } & 103\end{array}$

PART A: MiCRO-CANTILEVER INTEGRATED 2-D PHOTONIC CRYSTAL SLAB WAVEGUIDE FOR ENHANCED DISPERSION TUNING $\quad 104$

$\begin{array}{lll}6.1 & \text { INTRODUCTION } & 104\end{array}$

$\begin{array}{lll}\text { 6.2 } & \text { FABRICATION } & 106\end{array}$

$\begin{array}{lll}\text { 6.2.1 OPTICAL DEVICE FABRICATION } & 106\end{array}$

$\begin{array}{lll}\text { 6.2.2 MICRO-CANTILEVER INTEGRATION } & 107\end{array}$

$\begin{array}{lll}6.3 & \text { DISCUSSIONS ON FABRICATION } & 110\end{array}$

$\begin{array}{lll}\text { 6.3.1 MATERIAL SELECTION } & 110\end{array}$

$\begin{array}{lll}\text { 6.3.2 } & \text { EFFECTS ON CONFORMAL DEPOSITION } & 112\end{array}$

$\begin{array}{lll}\text { 6.3.3 PHOTOCHEMICAL ETCHING OF SILICON IN BHF } & 119\end{array}$

6.4 MECHANICAL CHARACTERISATION 119

$\begin{array}{lll}6.5 & \text { OPTICAL CHARACTERISATION } & 121\end{array}$

$\begin{array}{lll}\text { 6.6 CONCLUSIONS } & 123\end{array}$

PART B: MICRO-CANTILEVER INTEGRATED 2-D PHOTONIC CRYSTAL MICROCAVITY BASED DEVICE FOR WAVELENGTH TUNING 124

$\begin{array}{lll}6.7 & \text { INTRODUCTION } & 124\end{array}$

$\begin{array}{llr}6.8 & \text { FABRICATION } & 125\end{array}$

6.9 MECHANICAL CHARACTERISATION

6.10 OPTICAL CHARACTERISATION 130

$\begin{array}{lll}\text { 6.11 CONCLUSIONS } & 133\end{array}$

6.12 REFERENCES 133 
$\begin{array}{lll}7.1 & \text { CONCLUSIONS } & 136\end{array}$

$\begin{array}{lll}7.2 & \text { OUTLOOK } & 138\end{array}$

$\begin{array}{lll}7.3 & \text { REFERENCE } & 140\end{array}$

$\begin{array}{ll}\text { SUMMARY } & 141\end{array}$

$\begin{array}{ll}\text { SAMENVATTING } & 143\end{array}$

$\begin{array}{ll}\text { APPENDICES } & 145\end{array}$

$\begin{array}{ll}\text { I. FREEZE DRYING RELEASE } & 146\end{array}$

$\begin{array}{lll}\text { II. } & \text { Process DOCUMENTS } & 148\end{array}$

$\begin{array}{ll}\text { PUBLICATIONS } & 165\end{array}$

$\begin{array}{ll}\text { ACKNOWLEDGEMENT } & 167\end{array}$

$\begin{array}{ll}\text { BIOGRAPHY } & \mathbf{1 7 0}\end{array}$ 


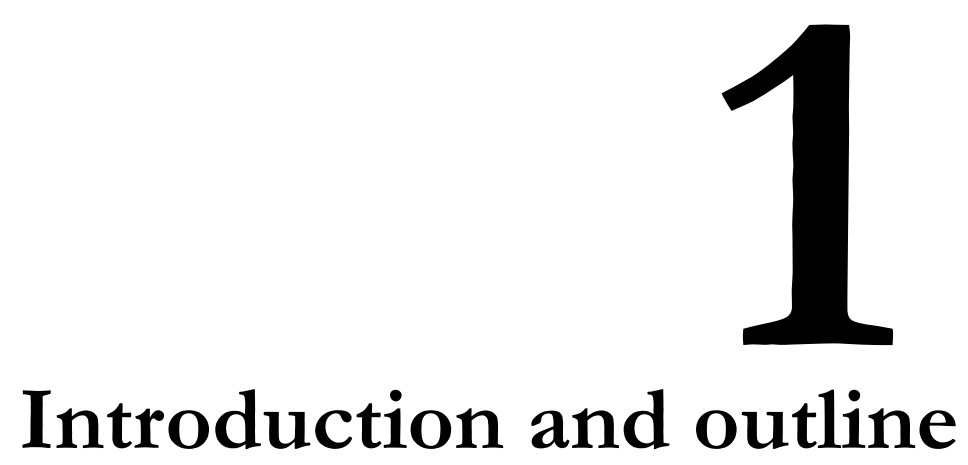

This chapter presents a general introduction to the subjects described in the thesis and to the research project. A brief introduction to three types of optical devices is presented: a microring resonator, a photonic crystal line-defect waveguide and a photonic crystal micro-cavity based device. The chapter ends with an outline of the thesis. 
Chapter 1. Introduction and Outline

\subsection{Introduction}

High speed data communication is today's expectation of virtually every single user connected to the internet. At present, long distance data communication is mostly realised employing, at least in part, optical fibre networks [1-2]. The data transmission through these fibres is in the form of modulated optical signals at carefully chosen wavelengths. At the network nodes, the optical signals need to be switched from one route to another which, at present, is achieved by electronic means. However, higher bandwidth can be achieved by replacing the electronic switches by appropriate optical devices. Keeping this goal in mind, scientists are working towards the realisation of optical components which eventually can be combined to form "photonic chips" [3]. Figure 1.1 shows an artists" impression of "The photonic chip" [3] in which many optical building blocks such as light sources, waveguides, switches, modulators and detectors are integrated on a single planar platform.

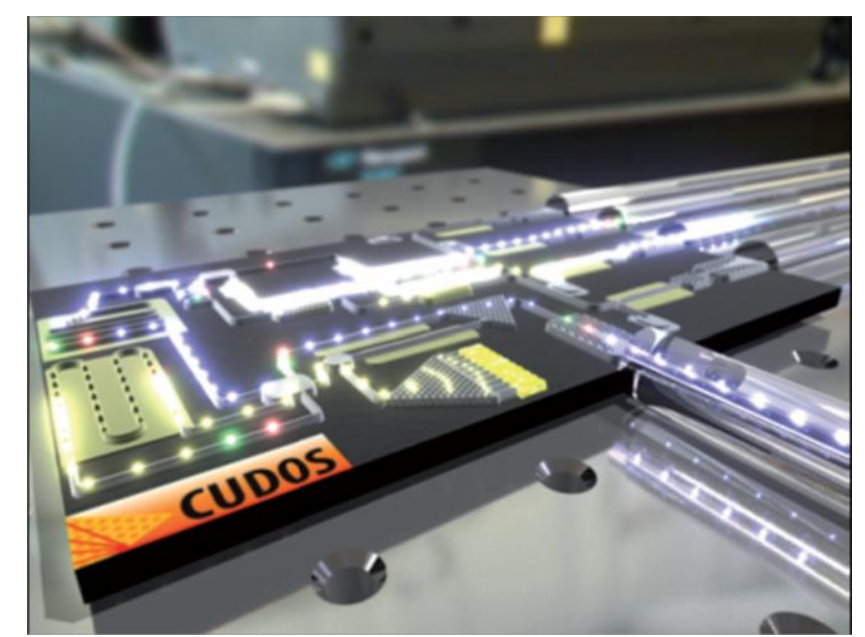

Figure 1.1. An artists' impression of a 'photonic chip' containing various integrated optical components in a planar platform. (Image source: [3]).

Apart from this, optical switches also can find application in short-distant on-chip interconnects. By realising electronic-photonic integration, the power loss produced by electrical interconnects can potentially be reduced. However this requires the development of complementary metal-oxide-semiconductor (CMOS) compatible technologies for electronic-photonic integration. Other than the aforementioned applications, optical switches can also be highly beneficial for (complex) sensing networks [4].

Silicon being the dominant material in the microelectronic industry, a highly matured technology has been developed based on it. This technology also can be fruitfully exploited for the realisation of silicon based optical devices. The additional 
benefit would be that silicon based optical devices can be relatively easily integrated with electronics, at least technology-wise. However, the impact on overall costs of such integration needs to be assessed, probably even on a case by case basis. Next to silicon, other materials such as lithium niobate $\left(\mathrm{LiNbO}_{3}\right)$ [5], gallium arsenide $(\mathrm{GaAs})$ and indium phosphide (InP), are studied. These materials lack the technological advantage of relative easy integration with CMOS fabrication, but offer their own strong 'selling points' in their material based suitability to fabricate light sources and detectors [6], second harmonic generation [7] and electro-optic [8] and electro-absorptive modulators [9], amongst others [10]. The key attractive features of silicon are its optical transparency in the near infra-red telecommunication wavelengths, possibility for mass production, low fabrication cost and ease in electronic-photonic integration. Keeping this in mind, the focus of this thesis is given to one technology, but with various device-concepts and implementations: 'siliconbased mechano-optical modulators.' ${ }^{1}$

One method to introduce modulation in a guided wave optical device is by changing the refractive index of one or a few of the materials through which the light propagates[11], which in turn changes the effective index and the field-distribution of the modes propagating in these waveguides. The refractive index of the materials of which the waveguides are composed can be changed by a variety of methods (which will be explained in section 2.1), one of which is by mechanically shifting pieces of material through the modal field distribution of the guided modes i.e. a mechano-optical effect. The focus of this thesis is given to technology for integration of such a mechanical element, a cantilever, with three different types of optical devices, as well as the subsequent characterisation of the integrated mechano-optical devices. The following section briefly discusses these three optical devices, which are a micro-ring resonator (MRR), a membrane-type 2-D photonic crystal slab line-defect waveguide (PCS-WG) and a membrane type 2-D photonic crystal slab micro-cavity based device (PCS-MC). Further, the purpose of this research is discussed in section 1.3 and an outline of the thesis is presented in section 1.4 .

1 The word 'modulator' shall be used in this thesis in the broader sense for any device that alters one or more properties of a propagating light beam (e.g. amplitude, phase, direction, polarization, frequency, propagation constant) and on any time scale versus the stricter use of the word as a device meant exclusively to impress information on a light beam and mostly referring to operation at signal bandwidth (sometimes as high as 10 - 100 's of $\mathrm{GHz})$. 
Chapter 1. Introduction and Outline

\subsection{Optical devices investigated}

\subsubsection{Micro-ring resonator (MRR)}

Micro-ring resonators are compact wavelength selective devices [12]. Over the past decade, they are extensively studied for realisation of various optical building blocks such as filters, multiplexers, modulators and switches [13]. Depending on the application, they can have a variety of geometries. A commonly used type is the so-called 'race-track' which is formed by increasing the coupling region between the a ring resonator and the waveguide by a straight piece. Figure 1.2A shows an SEM image of a three port, racetrack ring resonator with its access waveguides and (B) shows a schematic of the same. The working principle of an MRR is as follows [14]: when the optical signal passes through the In port, a part of the optical signal is evanescently coupled into the cavity whereas the rest of the signal appears in the Through port. The signal coupled into the cavity propagates around the ring and interferes with a later arriving part of the incoming signal from the In port. Destructive interference (of the cavity field and the field of the incoming signal) results in passing most of the light to the Through port. Constructive interference will result in most of the input power to circle the ring and eventually appear in the Drop port, again through evanescent coupling. In other words, light around resonant wavelengths appears as peaks in the Drop port and as dips in the Through port. Even though in an ideal case, at resonance, the achieved transfer efficiency can be $100 \%$, it is generally reduced in practice due to radiation losses inside the ring. The resonance wavelengths of the ring resonator $\left(\lambda_{\mathrm{m}}\right)$ are given by [15],

$$
\lambda_{\mathrm{m}}=\frac{L_{\text {ring }}}{m} n_{\text {eff }}
$$

Where $L_{\text {ring }}$ is the round trip length of the ring, $n_{\text {eff }}$ is the effective refractive index and $m$ is the cavity mode order. By mechano-optically perturbing, a change $\delta n_{\text {eff }}$ in the effective refractive index over a perturbation length $L_{\text {pert }}$ shifts the resonance wavelength by an amount $\delta \lambda_{\mathrm{m}}$ given by [15],

$$
\delta \lambda_{\mathrm{m}} \cong \frac{L_{\mathrm{pert}}}{m} \delta n_{\mathrm{eff}}
$$

Hence, the use of a mechano-optical modulator allows to adaptively and selectively change the value of the wavelengths that are removed from the Through port and transferred to the Drop port. 


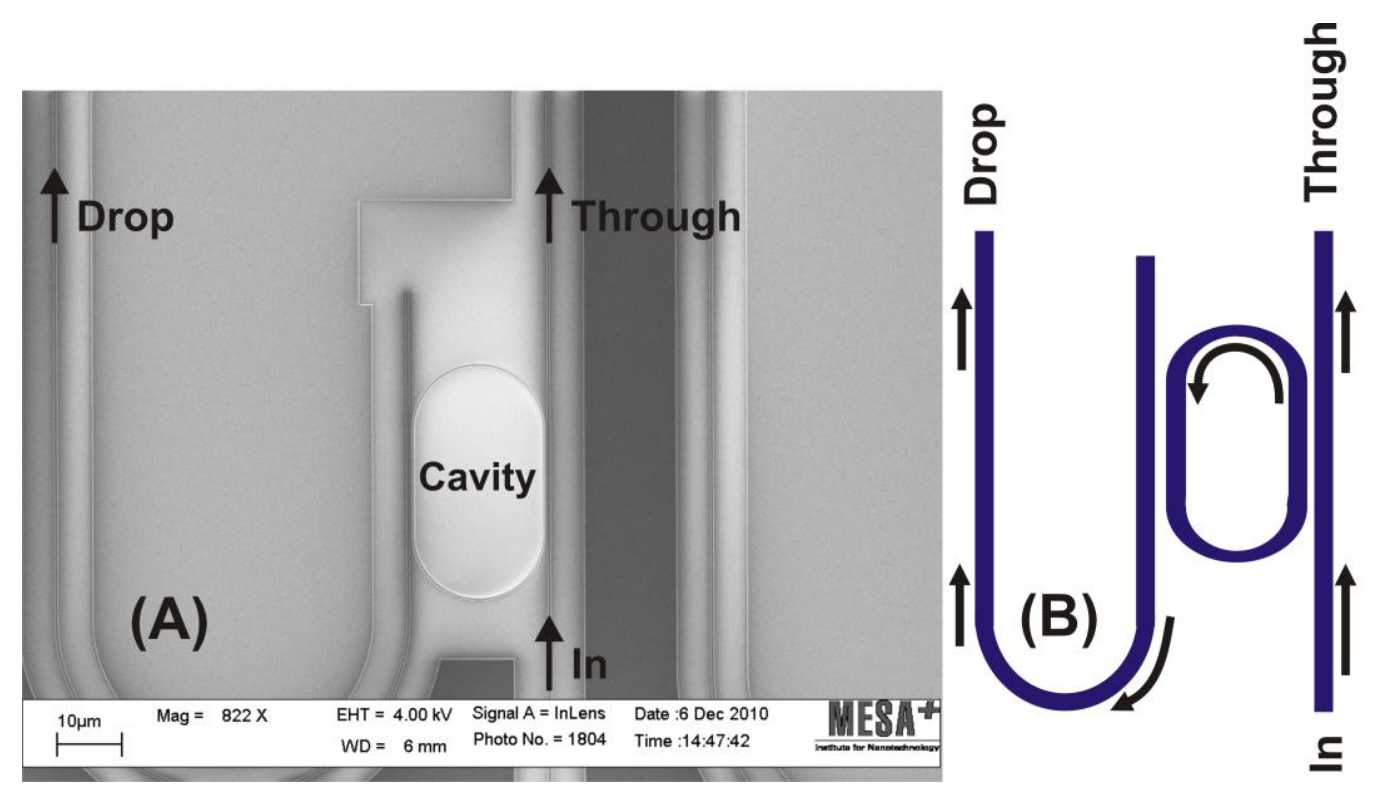

Figure 1.2. (A) SEM image of a race-track type micro-ring resonator with its access waveguides and (B) a schematic of the same.

\subsubsection{Photonic crystal (PC)}

Photonic crystals are engineered structures with a unique property for light transmission manifesting itself by the occurrence of a so-called 'photonic band gap'; a frequency range for which light propagation is inhibited [16-17]. Defect engineering in photonic crystal slabs (PCS) has paved the way to realise compact waveguides [18] and photonic circuitry, i.e. channel-drop filters (CDF) [19]. A so-called W1-type line-defect waveguide is realised by removing a row of holes such that light propagation is achieved by the combined effects of the photonic band gap (guidance in the plane of the waveguide) direction and by total internal reflection (guidance in the direction perpendicular to the slab). Figure 1.3A shows an SEM image of a 2-D silicon on insulator (SOI) based W1 photonic crystal slab waveguide (PCS-WG) having a triangular lattice of air-filled holes. A photonic crystal slab micro cavity (PCS-MC) based device is made using an optical resonator system, e.g. as shown in Figure $1.3 \mathrm{~B}$ by removal of 9 holes in between two symmetrically arranged modified W1-type waveguides. The micro-cavity provides some states in the photonic band gap of the W1 waveguides such that light of specific wavelength can be coupled to it and through to the adjacent waveguide. On proper design and fabrication, these devices can be used as wavelength dropping filters. A detailed description on the optical design and characterisation of these devices are given in [15]. 


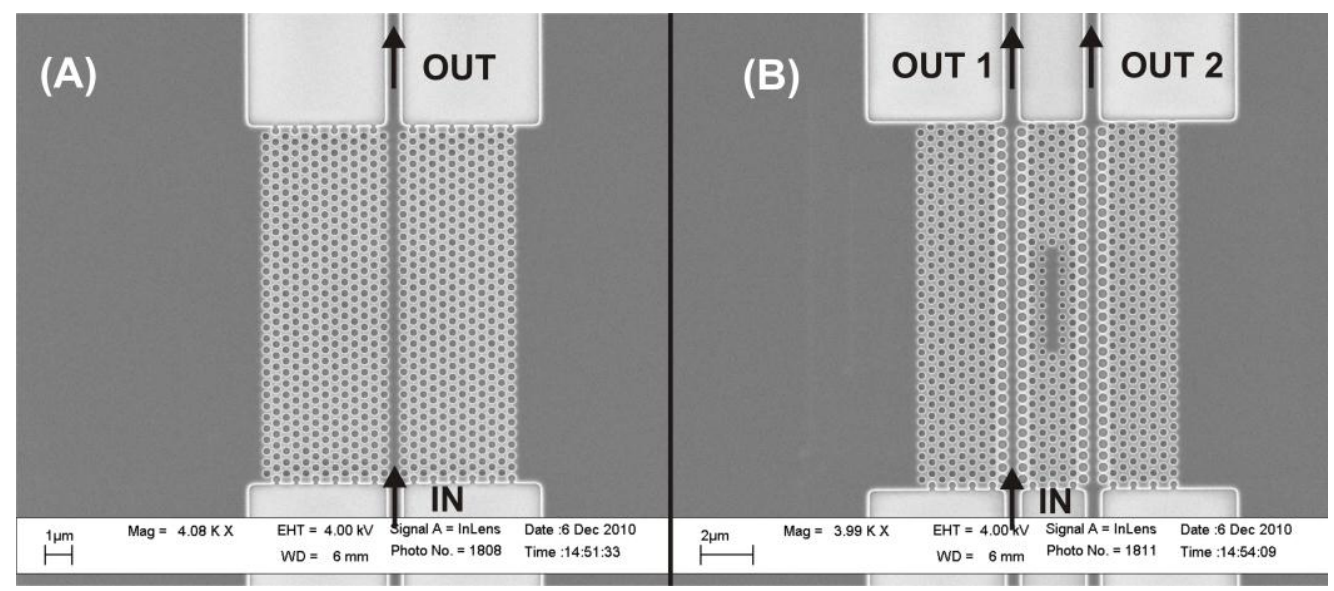

Figure 1.3. SEM images of $(A)$ a PCS line-defect waveguide caused by removing a single raw of holes (W1-type) and (B) a PCS micro-cavity based device formed by two symmetrically arranged modified W1type waveguides. These W1-type waveguides are separated by seven rows of holes and a cavity is formed in between them by omitting a row of nine holes. The periodicity of both the devices is $440 \mathrm{~nm}$.

\subsection{NEMS-project}

The research work reported in this thesis was funded by the nanotechnology investment programme NanoNed [20], by the Dutch ministry of economic affairs. The project entitled "Optical switching by NEMS-actuated resonator arrays" (TOE 7144: NEMS), which is part of a cluster of projects in the Nanophotonics flagship of NanoNed, aims to realise compact mechano-optical modulators for optical telecommunication networks. The other two projects are part of the cluster which were executed in the Applied Analysis and Mathematical Physics Group (TOE 7143, Modelling and Simulation tools which is concerned with the theoretical aspects of externally perturbed optical microcavities, and with the development of computational modelling and simulation tools that are suitable for the practical design of NEMS actuated resonator structures) and in the Integrated Optical Micro Systems Group (TOE 7145, Optics which aims at the realisation of compact optical resonator structures that have a good mechanooptical sensitivity and that are suitable for integration with NEMS actuators) of the University of Twente. The aim of the specific project TOE.7144 is to investigate the potential of MEMS-actuated wavelength-selective high-contrast optical devices such as micro ring resonators, photonic crystal line-defect waveguides and photonic crystal micro-cavity based devices. The relevance of the field of this study is exemplified by the number of publications produced over the past two decades (see Figure 1.4). The design and the characterisation of the optical devices were performed by a $\mathrm{PhD}$ researcher from the IOMS group whose work has recently be finalised in the form of his $\mathrm{PhD}$ thesis [15]. 


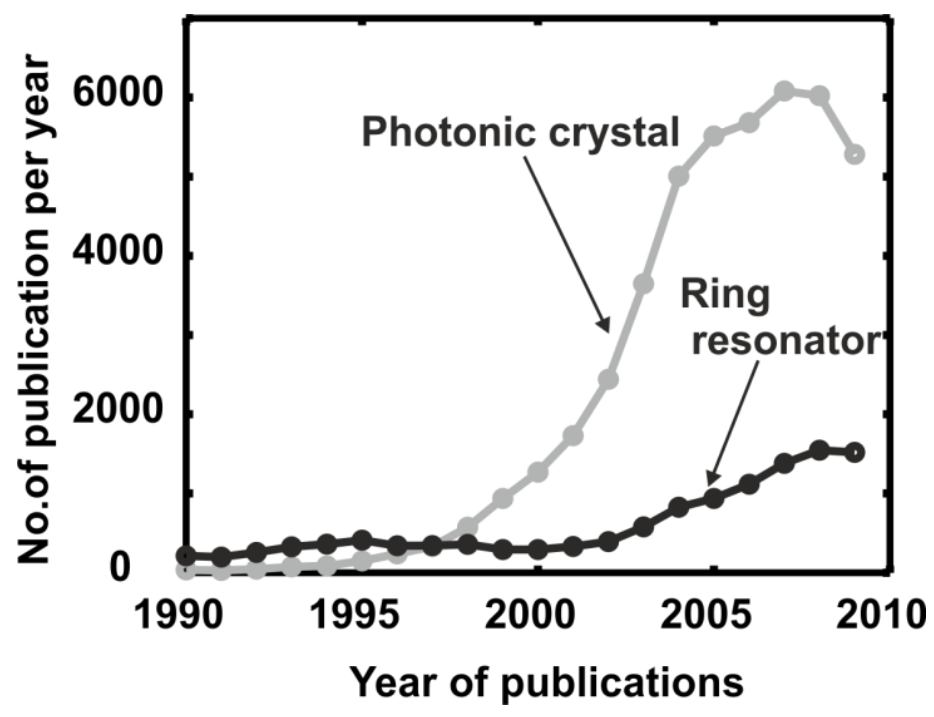

Figure 1.4. Number of publications per year related to 'photonic crystals' and 'ring resonators' (Extracted from Scopus [21] using the key words 'photonic crystals' and 'ring resonators')

The purpose of this research is to exploit nano-scale displacements of a dielectric mechanical element close to an optical device and, taking advantage of the small displacements, to aim at relative high modulation frequencies in the $\mathrm{MHz}$ range while still needing only modest actuation voltages. Micro-cantilevers are chosen as the mechanical elements. Owing to its low power consumption and relative ease of fabrication, electrostatic actuation was chosen to drive the cantilever. The optical devices were fabricated in a foundry process called ePIXfab (see section 1.3.1) using a silicon on insulator based high index contrast technology. Further processing only took place after the optical circuits were first characterised. In a custom process carried out in the MESA+ clean room, the micro-cantilevers were integrated with micro-ring resonators and PCS'. The latter were converted at selected areas into air bridge membranes, to retain the symmetry of the TE mode, by sacrificial etching of the buried oxide. Normally, integration with air bridge type PCS' requires complex fabrication schemes. But in this process we have successfully realised this by selectively removing the lower $\mathrm{SiO}_{2}$ cladding layer during one of the final etching steps. In PCS', apart from the evanescent field perturbation, direct interaction of the mechanical element with the optical field is achieved by micro-cantilevers equipped with tips that are self-aligned with respect to the holes of the PCS.

\subsection{1 ePIXfab: silicon photonics fabrication platform}

The optical devices described in this thesis were fabricated using the state-of-theart lithographic tools of the silicon photonics platform called ePIXfab [22] established at IMEC, Leuven. In this mask sharing activity, a designer is given a standard die area in the mask for the intended devices. Designs from several researchers are combined into an 
area (typically $12 \mathrm{~mm} \times 7.5 \mathrm{~mm}$ ) to form a master die. Sharing of the masks is motivated by the associated cost reductions. A $193 \mathrm{~nm}$ deep-UV lithographic process was used to realise the devices on 200-mm silicon-on-insulator wafers supplied by SOITEC [23]. The SOI wafer has a $220 \mathrm{~nm}$ thick silicon device layer, $2 \mu \mathrm{m}$ thick $\mathrm{SiO}_{2}$ lower cladding layer and a $700 \mu \mathrm{m}$ thick silicon handle wafer. The optical devices were fabricated by etching $220 \mathrm{~nm}$ deep into the silicon device layer [24]. A photograph of a processed 200-mm SOI wafer, before MEMS integration, is shown in Figure 1.5A. The wafer has a total of 186 dies. Figure 1.5B shows two dies of the wafer after MEMS integration. After removal of native oxide from the back side of the wafer, a piece of the SOI wafer was pasted on a copper plate with silver glue to have better electrical conductivity.
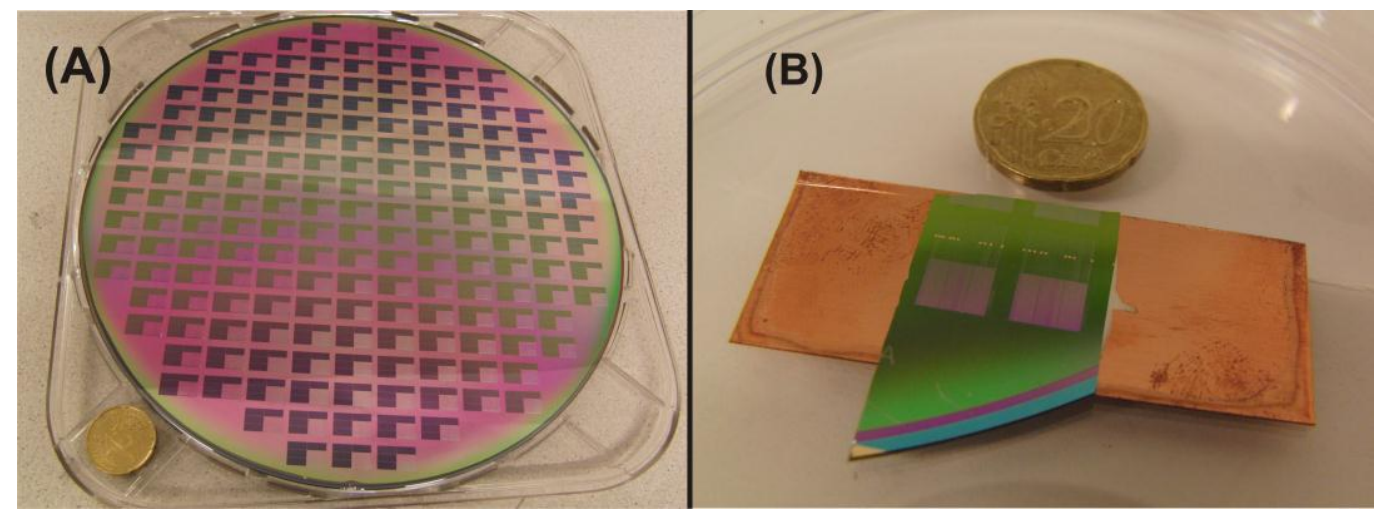

Figure 1.5. Photographs of (A) processed 200-mm SOI wafer before MEMS integration and (B) two dies on a piece of wafer after MEMS integration.

\subsection{Outline of the thesis}

The research carried out is presented in seven chapters in this thesis. As the title suggests, the thesis focuses on realisation of compact optical modulators based on monolithically integrated micro-cantilevers.

Chapter two presents a literature review of the various optical modulation methods related to micro-ring resonators, photonic crystal line defect waveguides and photonic crystal micro-cavity based devices. The relative advantage of mechano-optical interaction over other existing methods is highlighted in this chapter.

In chapter three, a systematic study of modelling, fabrication and characterisation of micro-curled bimorph cantilevers is described. Galerkin based static and dynamic models are used to predict various properties of the cantilever devices, such as off-state deflection, pull-in voltage, resonance frequency and electrostatic spring softening effects. Finally, the chapter describes design rules to optimise the performance of the cantilevers for mechano-optic modulation. 
Chapter four describes the fabrication technology for integrating bimorph cantilevers with micro-ring resonators. Various problems encountered during fabrication are discussed, in which a major one is related with the formation of etch residues at surface steps, called 'stringers'. A review of various existing stringer elimination methods in micromachining was carried out and is elucidated in this chapter. Further, the chapter proposes two alternative methods for stringer removal.

Chapter five describes the mechanical and optical characterisation of the microring resonator with integrated micro-cantilever. Various mechanical parameters of the micro-cantilever such as off-state deflection, pull-in voltage, resonance frequency, quality factor etc are measured and analysed. Further wavelength tunability and dynamic optical switching of the integrated devices are demonstrated.

Chapter six illustrates the technology for fabricating self-aligned tips with respect to holes of a photonic crystal slab waveguide and a photonic crystal micro-cavity based device. Various problems encountered during fabrication are discussed. A detailed analysis on the mechanical and optical performance of the integrated devices is carried out.

Finally, chapter seven presents the general conclusions of the thesis and provides recommendations for future research.

\subsection{References}

[1] J. Crisp and B. J. Elliott, Introduction to fiber optics. UK, 2005.

[2] J. M. Senior and M. Y. Jamro, Optical fiber communications: principles and practice, third edition ed. England: Pearson Education Limited, 2009.

[3] C. Grillet, et al., "Reconfigurable photonic crystal circuits," Laser \& Photonics Reviews, vol. 4, pp. 192-204, 2010.

[4] S. Janz, et al., "Chapter7-Silicon-based microphotonics for biosensing applications," in Optical waveguide sensing and imaging, ed: Springer, 2006, pp. 167-194.

[5] E. L. Wooten, et al., "A review of Lithium Niobate modulators for fiber-optic communications systems," IEEE Journal of Selected Topics in Quantum Electronics, vol. 6, pp. 69-82, 2000.

[6] M. S. Kumar, "Chapter5:Optical Sources and detectors," in Fundamentals of Optical Fiber Communication, ed New Delhi: Prentice-Hall of India Private limited, 2006, pp. 83-116.

[7] L. D. Malmstrom, et al., "Internal second-harmonic generation in Gallium Arsenide lasers," Journal of Applied Physics, vol. 35, pp. 248-249, 1964.

[8] R. Madabhushi, "Part 2: Optical Modulators,Chapter 6 - Lithium Niobate Optical Modulators," in WDM Technologies: Active Optical Components. vol. 1, ed: Elsevier 2002, pp. 207-248.

[9] T. G. B. Mason, "Part 2: Optical Modulators, Chapter 7 - Electroabsorption Modulators," in WDM Technologies: Active Optical Components. vol. 1, ed United States of America, 2002, pp. 249-314.

[10] J. E. Roth, et al., "Optical modulator on silicon employing germanium quantum wells," Optics Express, vol. 15, pp. 5851-5859, 2007.

[11] G. T. Reed, et al., "Silicon optical modulators," Nature Photonics, vol. 4, pp. 518-526, 2010. 
Chapter 1. Introduction and Outline

[12] B. E. Little, et al., "Microring resonator channel dropping filters," Journal of Lightwave Technology, vol. 15, pp. 998-1005, 1997.

[13] E. J. Klein, et al., "Densely integrated microring resonator based photonic devices for use in access networks," Optics Express, vol. 15, pp. 10346-10355, 2007.

[14] K. R. Hiremath, "Coupled mode theory based modelling and analysis of circular optical microresonators," PhD Thesis, University of Twente, 2005.

[15] L. J. Kauppinen, "Compact integrated optical devices for optical sensor and switching applications," PhD Thesis, University of Twente, 2010.

[16] E. Yablonovitch, "Inhibited spontaneous emission in solid-state physics and electronics," Physical Review Letters, vol. 58, pp. 2059-2062, 1987.

[17] S.John, "Strong localization of photons in certain disordered dielectric superlattices," Physical Review Letters, vol. 58, pp. 2486-2489, 1987.

[18] S. Noda, et al., "Full three-dimensional photonic bandgap crystals at near-infrared wavelengths " Science, vol. 289, pp. 604-606, 2000.

[19] S. Fan, et al., "Channel drop filters in photonic crystals," Optics Express, vol. 3, pp. 4-11, 1998.

[20] NanoNed. Available: http://www.nanoned.nl/

[21] Scopus. Available: http://www.scopus.com/home.url

[22] P. Dumon, et al., "Towards foundry approach for silicon photonics: silicon photonics platform ePIXfab," Electronics Letters, vol. 45, pp. 581-582, 2009.

[23] SOITEC. Available: http://www.soitec.com/en/index.php

[24] W. Bogaerts, "Nanophotonic waveguides and photonic crystals in Silicon-on-Insulator," $\mathrm{PhD}$ Thesis, Ghent University 2004. 


\section{2 \\ Mechano-optical modulators}

This chapter presents a brief overview of past and existing efforts towards the tuning and switching of integrated optical devices. The merits of mechano-optical modulation schemes are highlighted. The analysis is focused on three different types of optical devices: a micro-ring resonator, a photonic crystal slab line-defect waveguide and a photonic crystal micro-cavity based device. 
Chapter 2. Mechano-optical modulators

\subsection{Various optical modulation schemes: previous works}

Refractive index variations in optical devices can be achieved in a variety of ways. Among them, most commonly employed principles are thermo-optic effects [1], electrooptic effects [2], liquid crystal rotation [3] and mechano-optical effects [4]. The following sub-sections give a brief overview of these modulation methods as used in silicon based optical devices. Highlight of mechano-optical modulation schemes are given in section 2.1.5.

\subsubsection{Thermo-optic effect}

As the refractive index of a material is dependent on temperature, a variation in temperature can be utilised to realise temperature-induced optical modulation. Owing to the high thermo-optic coefficient of silicon $\left(\sim 2.4 \times 10^{-4} \mathrm{~K}^{-1}[5]\right)$, thermo-optic modulation is often used in silicon based optical devices. However, the drawbacks of this method are the slow switching speed, (typically on the order of $0.1 \mathrm{~ms}$ in silicon nitride based optical devices [6] and $1 \mu \mathrm{s}$ in silicon based devices [7]) and the high power consumption (amongst others due to the high thermal conductivity of silicon of $\sim 150 \mathrm{WM}^{-1} \mathrm{~K}^{-1}$ [8]). Power consumption is even more severe when devices are used in arrays, moreover posing cross-talk problems between adjacent devices. Even though being reversible in nature this effect is not preferred if localised tunability is a key requirement. Figure 2.1 left shows an image of an integrated heater with a PCS micro-cavity, used for thermo-optic modulation [9].

\subsubsection{Electro-optic effect}

Some materials, e.g. the class of non-centrosymmetric materials, offer possibilities for optical modulation by exhibiting a dependence of the refractive index on (applied) electrical fields: the electro-optic effect. A well-known material used for realising electrooptical modulation is Lithium Niobate. Although the attainable refractive index changes are generally small compared to thermo-optic effects, the attractive features of electrooptic modulation is the low power consumption and nanosecond switching times [10]. But in silicon, as the linear (Pockels) electro-optic effect [11] is non-existent and the quadratic (Kerr) effect [12] is weak, electro-optic modulation is less preferred. 

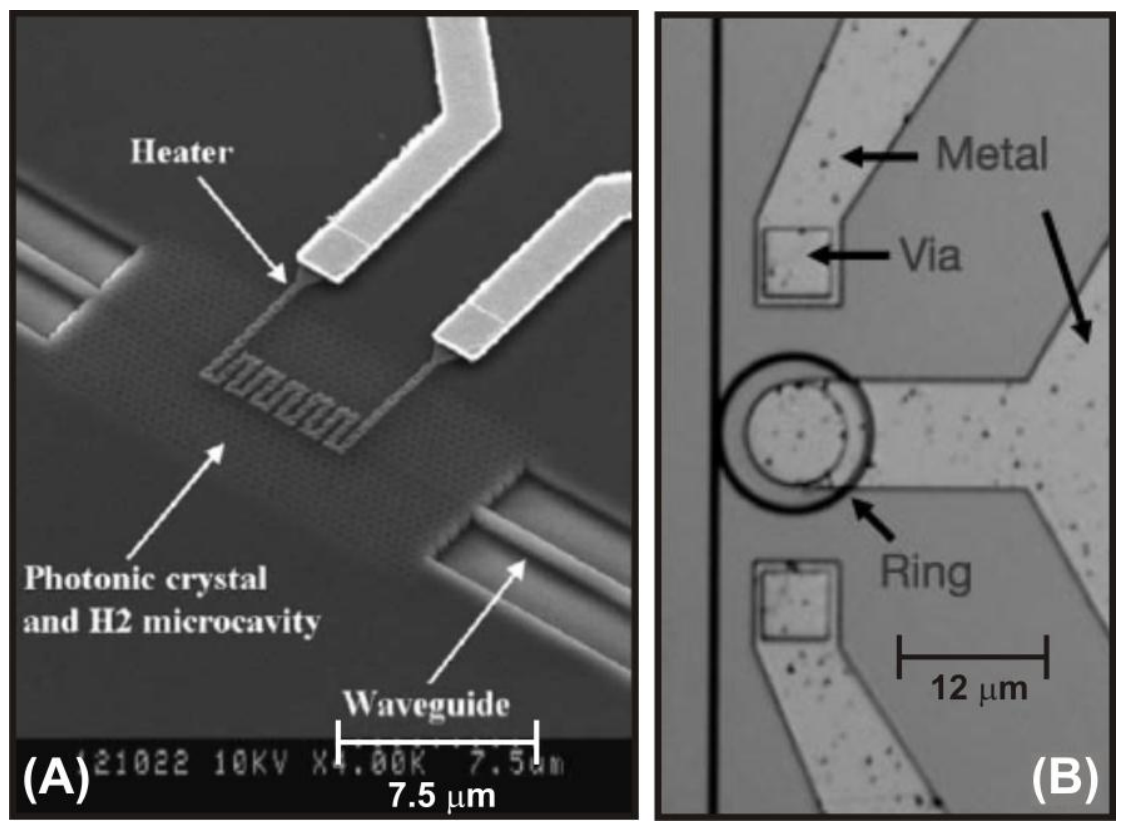

Figure 2.1. Images showing (Left) integrated heater for thermo-optic modulation in a PCS micro-cavity (Image source: [9]) and (Right) modulation by charge injection effects in a micro ring resonator (Image source: [13]).

\subsubsection{Plasma-dispersion effect}

The plasma dispersion effect is related to changing the concentration of free charge carriers in a semiconductor material, which in turn changes both the real and imaginary parts of its refractive index [14]. The carrier concentration in silicon can be modulated by a variety of methods such as injection, accumulation, depletion or inversion of carriers. Even though this method gives the possibility for localised tuning and yields fast $(>1 \mathrm{~ns})$ and low power $(\sim 10 \mu \mathrm{W})$ [13] modulation schemes, the approach is limited due to relative large optical absorption losses. Figure 2.1 right shows a micro-ring resonator, carrier injection based modulator [13].

\subsubsection{Liquid crystals infiltration}

The effective refractive index of an optical guided mode can also be varied using (materials infiltrated by) liquid crystals. Due to their specific ordering these materials are generally birefringent and their 'liquid' property allows to rotate the optical axis of the material introducing changes in the refractive index. To this respect, liquid crystals (LC) are exploited of which the refractive index can be changed by either temperature [3] or by an applied electric field [15]. But it is noteworthy that in this method, the tunability of the device is dependant on the birefringence of the LC material. Apart from this, LC orientation rotation is inherently slow, typically in the order of milliseconds but this can be downscaled to micro-seconds when used with special ferroelectric or polymer 
Chapter 2. Mechano-optical modulators

dispersed LCs [16]. Another drawback is its liquid state which can limit compatibility with other processing steps or even restrict it for use in specific applications.

\subsubsection{Mechano-optical interaction}

Introduction of a mechanical element into the optical field can affect the effective refractive index of a guided mode. In mechano-optical modulation schemes the perturbing element can either be introduced into the optical path or into the optical evanescent field [17]. The following sub-sections provide a detailed description of mechano-optical modulation achieved so far in optical devices such as MRR, PCS-WG and PCS-MC. Section 2.1.5.1 briefly describes the mechano-optical modulation scheme realised by application of mechanical stress into an optical device. Optical field interaction by external tips is presented in section 2.1.5.2 while efforts to realise compact MEMS integrated optical modulators are discussed in section 2.1.5.3. Devices comprising of selfaligned tips with respect to holes of PCS's are discussed in section 2.1.5.4.

\subsubsection{Applying mechanical stress}

Mechanical deformation of materials generated by force/stress induced by an external agent has been used to achieve tunability, for example in photonic crystals (PC). Stress induced spectral tuning, by deformation of a micro-bridge on which a 1-D PC is fabricated is demonstrated by Rajic et al. [18]. Tuning of an SOI based photonic crystal slab (PCS) micro-cavity, through changes of its dimensions by an integrated, comb drive actuated, flexure is proposed by Levy et al. [19] and the concept is later demonstrated in a polymer based PCS by Park and Lee [20-21]. Recently Shambat et al. presented a membrane GaP-PCS micro-cavity device where a shift in cavity resonance wavelength could be induced by deforming the membrane by a fibre that was simultaneously used to achieve optical coupling to the device. The shift was the result of the combined effects of cavity mode perturbation, stretching of the PC membrane and the photo elastic effect [22-23]. The drawbacks of this method are its non-localised effects and limited suitability for materials other than polymer based PCs (in which the applied stress can be translated to a large change in mechanical properties).

\subsubsection{External perturbation by AFM/SNOM/fibre tips}

Perturbation of the evanescent optical field of guided modes by moving nanometric tips has achieved some interest in recent year due to the prospect of locally modifying the guided mode properties. Resonance wavelength tuning of MRR and PC micro-cavities is demonstrated using various perturbing shapes and materials such as the tip of an atomic force microscope (AFM made up of silicon [24-26] and $\mathrm{Si}_{3} \mathrm{~N}_{4}$ [26]), fibres (silica [27]) and the tip of a scanning near-field optical microscope (SNOM made up of 
silica [28] and dielectric material coated with chromium-gold bilayer [29]). Figure 2.2A shows a schematic representation of an AFM cantilever aligned on top of cavity and (B) shows an SEM image of an AFM cantilever with its tip at the end. Rakich et al. [27] used a silica fibre probe to perturb the evanescent field of an air-clad silicon-nitride MRR. This allowed to increase the effective index of the mode resulting in a $27 \mathrm{~nm}$ wavelength shift of the initial resonance wavelength at $1565 \mathrm{~nm}$. AFM cantilevers have been used as well to tune the transmittance of a 2-D photonic crystal reflector [25] and the resonance of a PCS micro-cavity [24, 26]. Further SNOM tips have been used to alter the modal properties of PCs [28-29]. The major challenge in the implementation of this kind of modulation scheme is the required alignment between the perturbing body and the optical device. Especially in the case of photonic crystals were the hole diameters are typically on the order of a few hundreds of nanometres, precise aligning is very critical. Moreover the difficulty increases if more than one tip needs to be aligned on top of the holes. Except in demonstrating the feasibility of the principle, this method is inadequate to be used for a compact integrated optical device.

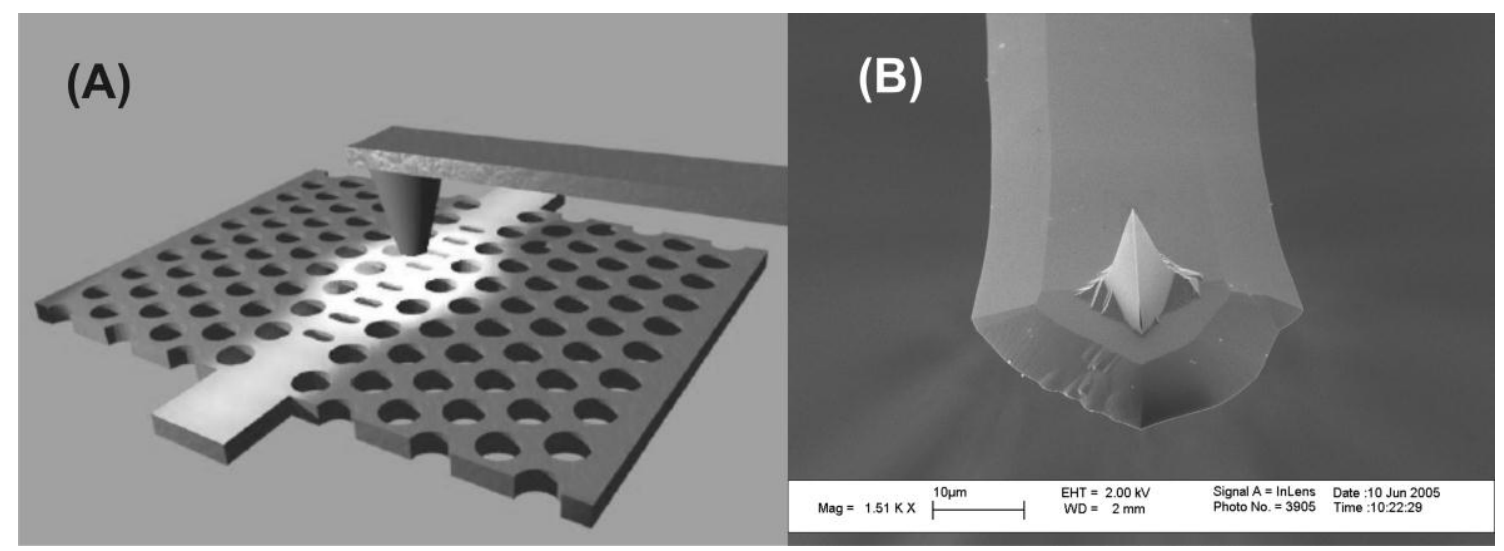

Figure 2.2. (A) Schematic showing an AFM cantilever in the close proximity of a PC micro-cavity (Image source: [24]) and (B) SEM image of an AFM cantilever with its tip (Image source: M.H. Siekman, TST Group, University of Twente, The Netherlands).

\subsubsection{Perturbation by integrated MEMS}

The concept of MEMS integrated optical devices, utilising the mechano-optic effect has been introduced a few years back [30], amongst others by Lukosz [31]. Meanwhile MEMS integration has been studied on various optical devices. The integration between an optical device and a mechanical element can be done either monolithically or by hybrid methods. In monolithic integration, the final device is realised from a single wafer (with the help of sacrificial layer etching and few other thin film technologies) whereas in hybrid integration, different components are fabricated 
separately on different wafers which are later bonded to each other. The supremacy of one method over the other is debatable.

Among the various MEMS actuation schemes, electrostatic actuation [32] has distinct advantages over other methods: low power consumption, ease of fabrication, ease of control, etc.. Hence an electrostatic actuation scheme is exploited in many of these devices. A hybrid integrated, electrostatically actuated, absorptive on/off intensity modulator is demonstrated by Veldhuis et al. [4, 33] at $632.8 \mathrm{~nm}$. The device consists of a mechanical element that due to its interaction with the optical evanescent field of a waveguide, absorbs the propagating guided mode. Various implementations, with response times of $\sim 10 \mathrm{~ms}, \sim 1 \mathrm{~s}$ and $\sim 10 \mathrm{~s}$ and between 40 and $65 \mathrm{~dB}$ extinction ratio were demonstrated. In a different approach, a monolithically integrated and electrostatically actuated comb drive is used by Bulgan et al. [34] to open/close a section of a straight waveguide. The device with an active switching region as small as $40 \mathrm{x} 60 \mu \mathrm{m}^{2}$ (Figure 2.3A) is used as an on/off switch having an extinction ratio of $15 \pm 2 \mathrm{~dB}$ for a wavelength of $1.55 \mu \mathrm{m}$. A MEMS integrated wavelength selective switching device using a micro-ring resonator is demonstrated by Nielson et al. [35]. The integrated device consists of an absorptive aluminium membrane (with etch holes as seen in Figure 2.3B) introducing $15 \mathrm{~dB}$ extinction of the light signal in its drop port. Switching rates of up to 8 $\mathrm{kHz}$ with $30 \mathrm{~V}$ square wave signals were demonstrated as well. The aforementioned devices [4, 33, 35] are based on absorption modulation schemes. These schemes are unsuitable for wavelength tuning since they effectively modulate by dissipating the incoming light.

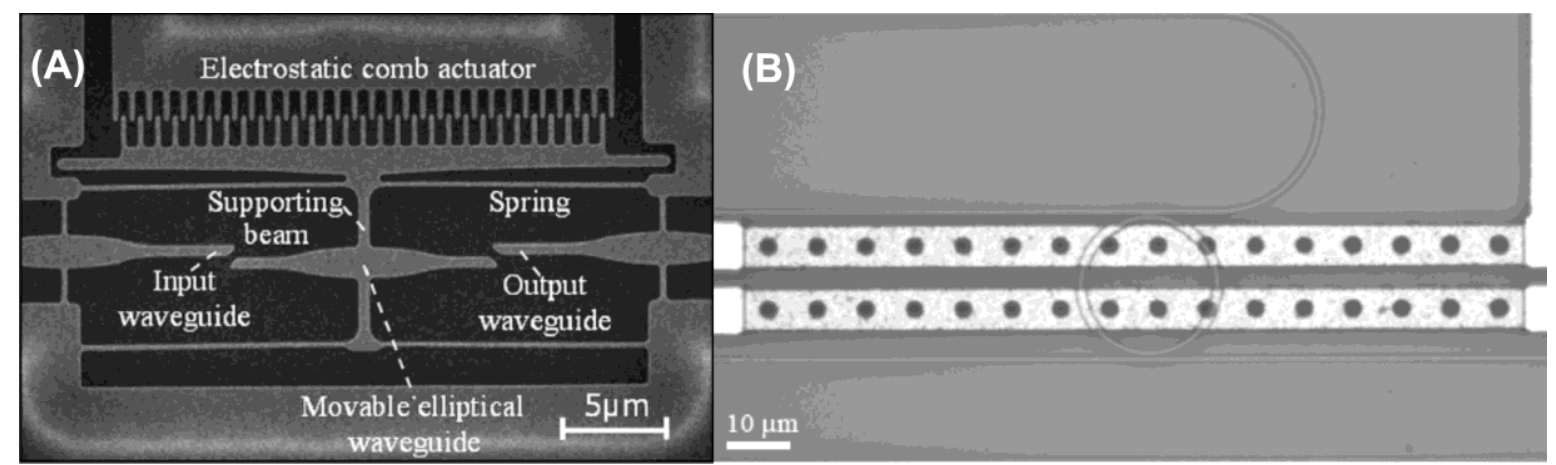

Figure 2.3. SEM images showing electro-statically actuated (A) comb drive integrated with a straight waveguide (Image source: [34]) and (B) an absorptive clamped-clamped beam integrated with a micro-ring resonator (Image source: [35]).

\section{1-D Photonic crystals}

Albeit there are many numerical studies and proposed designs regarding the integration of MEMS with PC based devices, only few works have reported successful integration and 
its experimental demonstration. The first fabrication of an integrated device for on/off switching in 1-D PCs is reported by Lee et al. [36] which shows $11 \mathrm{~dB}$ extinction ratio at $1.56 \mu \mathrm{m}$ and $0.56 \mathrm{~ms}$ time constant. Although named 1-D PC, this optical device consists of only two silicon slabs with an air gap in between them (Figure 2.4A). An electro-static comb-actuated slider is inserted into this air gap to get either a reflection or transmission state, where the device operation concept is similar to that described by Bulgan et al. in [34]. In 2010, dynamic tuning of the resonance of a comb-actuated 1-D free-standing PC nano-cavity has been demonstrated by Chew et al. [37] with a resonance wavelength tuning of the even mode by $2.6 \mathrm{~nm}$ and the odd mode by $1.75 \mathrm{~nm}$ (Figure 2.4B). Further it was shown that modulation of PC-nanocavities is highly dependent on the geometry and size of the perturbing tips, using various structures such as nano-tips, meniscus liketips etc [38].
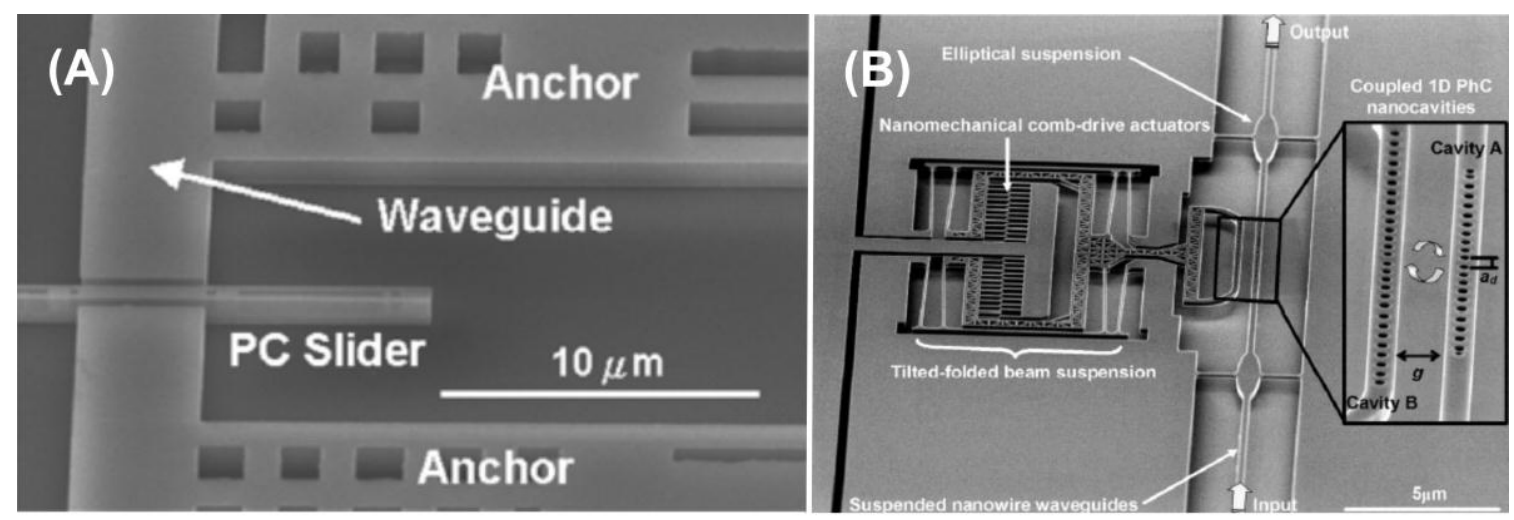

Figure 2.4. Electrostatically actuated MEMS integrated 1-D PC modulators based on (A) waveguide (Image source: [36]) and (B) nanocavity (Image source: [37]).

\section{2-D Photonic crystals}

In 2003, Suh et al. [39] theoretically showed that the transmittance and reflection coefficients of light under normal incidence to a PC slab can be varied by coupling two PC slabs parallel to each other. In this approach, strong variation in transmission coefficient is achieved by nano-scale variation in the spacing between the two PC slabs. A year later, optical switching in a line-defect waveguide was experimentally demonstrated by Iwamoto et al. [40-41] by vertically moving a dielectric plate (GaAs, 60 x $120 \mu \mathrm{m}$ ) by an 'external' piezo-electric actuator towards the waveguide. The GaAs plate, upon actuation, changed the effective index of the propagating light wave and evanescently coupled it into the plate. Even though the device was not integrated, an extinction ratio of $\sim 10 \mathrm{~dB}$ was experimentally demonstrated. A PCS based optical filter, with normal incident light, which can control the reflectivity based on evanescent coupling was demonstrated by Kanamori et al. [42]. In this work, the guided resonance of the movable 
SOI based PCS was controlled by changing the gap between the PCS and the substrate by means of integrated electro-thermal MEMS actuators.

The first experimental study on integrating micro-cantilevers with a PCS-WG is reported by Kanamori et al. [43]. The finite difference time-domain (FDTD) method was used to calculate the optimal parameters for a device in which an electrostatically actuated poly-silicon cantilever, having silicon rods at its end, is used to fill the holes of a PCS-WG in order to introduce crystal defects. However, the device lacks any optical characterization till to date. The first successful device, with actuating element integrated with a PCS, and which was optically characterised as well, is reported by Iwamoto et al. [44-45]. In this case, an electrostatically actuated poly-silicon cantilever is integrated with a SOI based PCS-WG (Figure 2.5A). A maximum extinction ratio of $10 \mathrm{~dB}$ with a voltage as high as $60 \mathrm{~V}$ and optical modulation with a $0-60 \mathrm{~V}$ square wave at $10 \mathrm{~Hz}$ is demonstrated. Since the dielectric layer (poly-silicon) of the micro-cantilever has a higher refractive index than the smallest effective refractive index of the PCS guided light, upon insertion the guided light is coupled to the dielectric layer and subsequently lost. Hence the device cannot be used for optical phase modulation.

A different type of MEMS integrated 2-D PC-WG is reported by Umemori et al. [46]. This device consists of three parts: an input and output silicon PCS in which a linedefect waveguide is formed (see Figure 2.5B). The third part is a central moving silicon slab which is electrostatically actuated to act as the switching element between the input and output waveguides. The light is propagated when the slab is at the same level as the input and output waveguides and the propagation is decreased by moving the slab downward. A relatively small minimum modulation of $4 \mathrm{~dB}$ was experimentally demonstrated by applying a voltage as high as $180 \mathrm{~V}$. Light transmission was not completely extinguished when the slab was moved further downward but rather increased on increasing voltage reaching a plateau of about $-2 \mathrm{~dB}$.
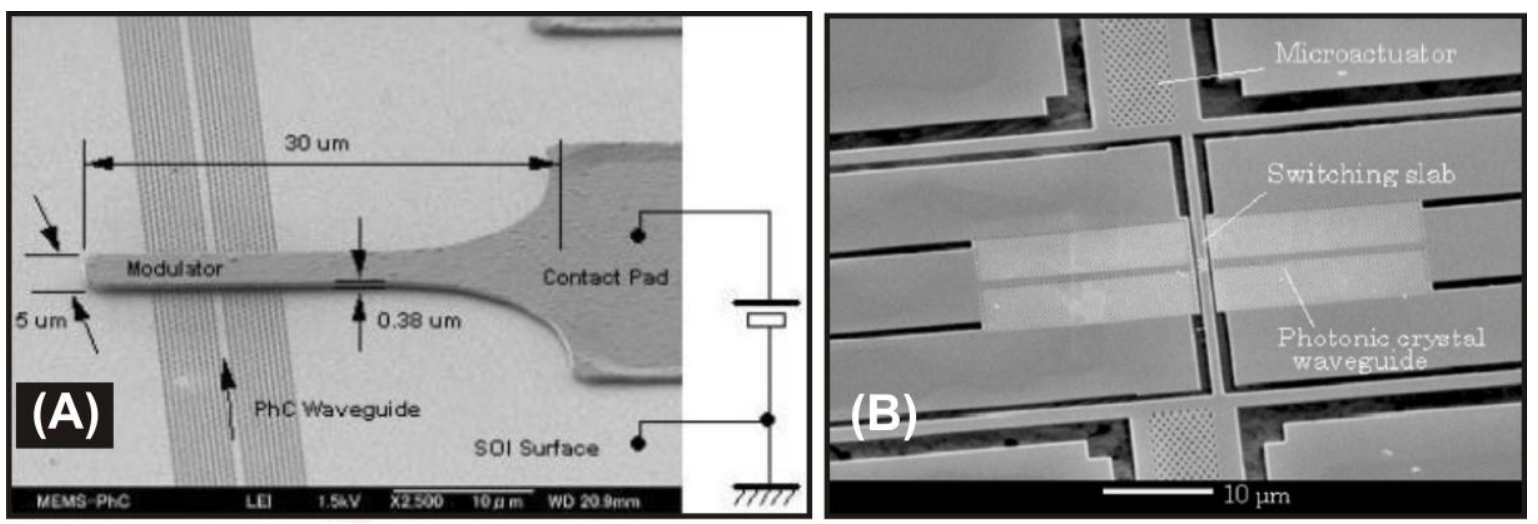

Figure 2.5. Electro-statically actuated MEMS integrated 2-D PC-WG modulators based on (A) (Image source: [44-45]) and (B) (Image source: [46]). 
In 2009, aiming at realisation of a wavelength selective channel-drop filter, mechanical tuning of a PC nanocavity by an integrated electrostatic comb actuator was demonstrated by Kanamori et al. [47]. The device consists of a PCS-WG with its waveguide at the edge of the slab and a movable PC-nanocavity. The drop efficiency between the PCS-WG and the PCS nanocavity is increased by decreasing the gap between them (by $12.5 \mathrm{~dB}$ for a gap change of $600 \mathrm{~nm}$ ).

\subsubsection{Insertion of self-aligned tips into the PC holes}

Apart from the evanescent field perturbation, there have been few works where the mechanical perturbing element is introduced directly into air holes of a PCS structure. It has been achieved by inserting self-aligned tips (made from the lower cladding material) into the PC holes. A reconfigurable PCS-WG fabricated on GaAs in which a MEMS cantilever ( $\mathrm{Al}_{\mathrm{x}} \mathrm{GaAs}$ ) generates/cancels a section of a waveguide by insertion of selfaligned tips is demonstrated by Zhou at al. [48] (see Figure 2.6A). Though the device is successfully fabricated, optical modulation measurements have not yet been reported. In a similar approach Takahata et al. [49] demonstrated that the transmittance of a membrane SOI type PCS-WG can be changed by inserting self-aligned tips into each of the PCS holes (see Figure 2.6B). By electrostatically actuating the SOI based PCS membrane, extinction ratio, of the order of $-4.4 \mathrm{~dB}$ is demonstrated in the telecommunication wavelengths. In both the approaches the PC layer and the bottom cladding layer is utilised for fabricating self-aligned tips, using electron beam (EB) lithography and selective etching. Hence the tips have two parts: the top part made of the PC layer material and the bottom part made up of bottom cladding layer. As a possible disadvantage it is remarked that the device was made by EB lithography which is laborious and expensive for large scale integration.
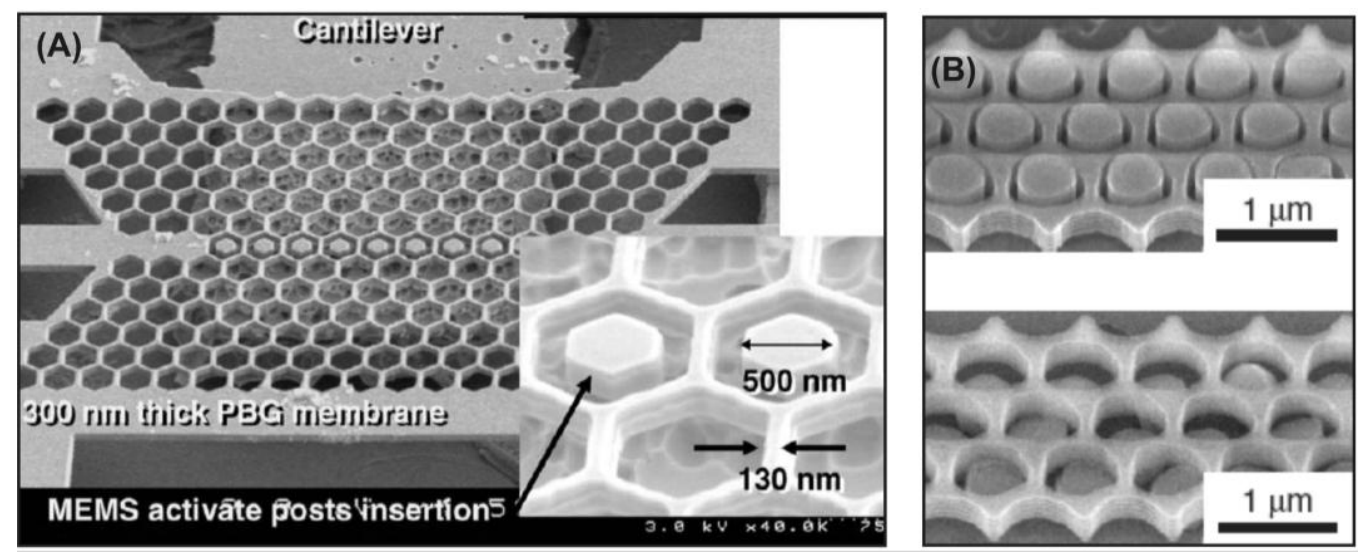

Figure 2.6. SEM images showing self-aligned tips (A) on an integrated cantilever which are inserted into the PC holes (Image source: [48]) and (B) selectively etched from the bottom cladding layer which are inserted in (top) and out (bottom) of the PC holes (Image source: [49]). 
Chapter 2. Mechano-optical modulators

\subsection{Our approach}

The research focus of this thesis is to realise compact integrated wavelength selective devices exploiting the mechano-optical modulation scheme. Due to its limited fabrication complexity and low power consumption, a vertical electrostatic actuation scheme is chosen. Although piezoelectric actuators would have many attractive features we have disregarded this possibility due to the issues related to integration of piezoelectric materials in a surface micromachining process [50]. Moreover we disqualified electrothermal actuation scheme on the basis of anticipated longer time-responses. Compared to e.g. comb drive electrostatic actuation, micro-bimorph cantilever based devices have the potential for higher mechanical resonance frequencies and device compactness. As in our design the distance between two adjacent optical devices is $<100 \mu \mathrm{m}$, and typically comb drive actuators have a large foot print compared to micro cantilevers, the former one is discarded for the current study.

So far only intensity modulation has been reported in micro-ring resonators using an integrated mechanical element. Even though the wavelength tunability has been demonstrated by an external mechanical perturbing element, no study has been carried out in realising integrated devices. To achieve wavelength tuning without incurring or changing optical losses, the mechano-optical perturbing element must be a dielectric and have a refractive index lower than the refractive index of the optical device layer. Since silicon on insulator based optical devices are our key interest of research, silicon nitride is chosen as the material for the cantilever / mechano-optical perturbing elements. Owing to its low stress compared to stoichiometric silicon nitride, silicon rich nitride is considered for the current study.

Another goal is to develop a simple technology to fabricate self-aligned tips with respect to the nanometer scale holes of a photonic crystal (PC) using conventional lithography. Since an optical evanescent field decays exponentially from the photonic device, in order to have a large mechano-optic effect, the perturbing element needs to be able to be manoeuvred into this evanescent field and as close as possible to the waveguiding structure. Compared to a flat cantilever, a cantilever with self-aligned tips can penetrate into the PC holes resulting in larger mechano-optical effects. If a regular lithography process would be used to fabricate these tips it would need a) critical smallest dimensions for the tips to fit into the holes and b) critical alignment for the tips to eventually sit above the holes. Here we demonstrate a simple fabrication scheme, using conventional lithography, to realise this while preventing the need for precise nanometric alignment steps. The idea is to deposit a sacrificial layer, which has a thickness less than the radius of the PC hole, so that the hole is not completely filled by the sacrificial layer (see Figure 6.5). Thus the subsequently deposited cantilever device layer penetrates into 
the PC hole, which on sacrificial layer etching results in the formation of tips, self-aligned with respect to the PC holes. Since a thin sacrificial layer is the primary requirement of this process, fabricating a straight cantilever would result in perturbation of the optical properties even in the off-state. To circumvent this problem, the cantilever is designed to be curled upward in its off-state which is realised by carefully optimising the stress in the cantilever layer. However, the curling should be well-controlled and limited (acceptable is $<500 \mathrm{~nm})$ as increased curling would result in higher voltage requirements to manoeuvre the beam into the optical field. Figure 2.7A shows a schematic of a straight cantilever with (too) thick sacrificial layer, which would result in a large air-gap. Worse, even though the optical evanescent field is unaffected in the cantilever off-state, the process will not yield self-aligned tips. Figure $2.7 \mathrm{~B}$ shows the case of a reduced sacrificial layer thickness $\left(t_{\mathrm{SL}}\right)$ where the cantilever is designed to have an acceptable off-state deflection. This design will not affect the optical properties in the off-state and will still result in self-aligned tips. A detailed theoretical and experimental analysis to optimise the off-state deflection and other cantilever properties is given in the next chapter.

(A)

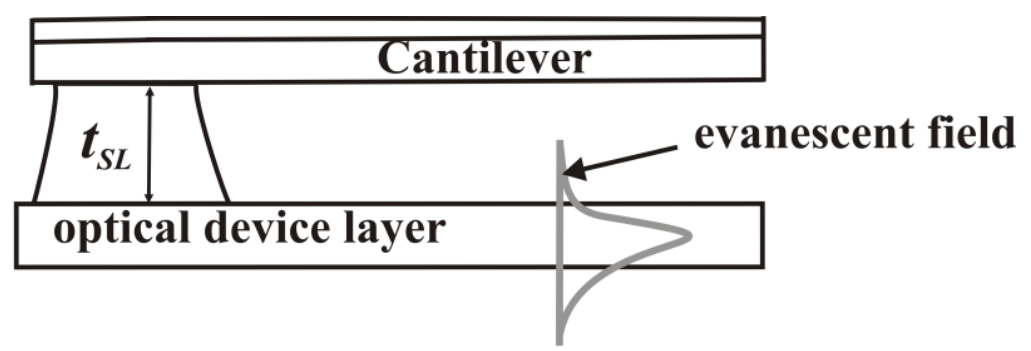

(B)

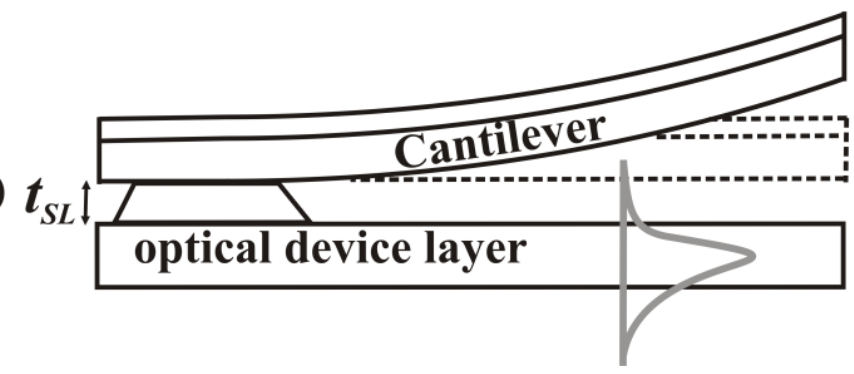

Figure 2.7. Schematic representing (A) a straight cantilever integrated with an optical device having a thick sacrificial layer which results in a large air-gap. For this case, the optical evanescent field is unaffected in the cantilever off-state but self-aligned tips cannot be made. (B) Shows the case of a thin sacrificial layer which does allow the formation of self-aligned tips. If the cantilever would be straight in this case, the optical evanescent field would be affected in the cantilever off-state; hence upward curling is preferred to increase the modulation depth.

\subsection{Conclusions}

A general introduction to various topics discussed in the thesis is presented in this chapter. A background study related with various optical modulation schemes in silicon 
Chapter 2. Mechano-optical modulators

based optical devices is presented. The relative advantages of mechano-optical modulation schemes compared to other methods are highlighted. The analysis focuses on three different types of photonic devices: a micro-ring resonator, a photonic crystal linedefect waveguide and a photonic crystal based micro cavity resonator.

\subsection{References}

[1] G. Cocorullo, et al., "Thermo-optic effect exploitation in silicon rnicrostructures," Sensors and Actuators A, vol. 71, pp. 19-26, 1998.

[2] R. Madabhushi, "Part 2: Optical Modulators,Chapter 6 - Lithium Niobate Optical Modulators," in WDM Technologies: Active Optical Components. vol. 1, ed: Elsevier 2002, pp. 207-248.

[3] D. M. Wang B, Silov AY, Nötzel R, Karouta F, He S, van der Heijden RW., "Controlling mode degeneracy in a photonic crystal nanocavity with infiltrated liquid crystal," Optics Letters, vol. 35, pp. 2603-2605, 2010.

[4] G. J. Veldhuis, et al., "Mechano-optical waveguide on-off intensity modulator," Optics Letters, vol. 23, pp. 1532-1534, 1998.

[5] M. T. Tinker and J.-B. Lee, "Thermo-optic photonic crystal light modulator," Applied Physics Letters, vol. 86, pp. 221111-1-3, 2005.

[6] E. J. Klein, "Densely integrated microring-resonator based components for fibe-to-thehome applications," PhD Thesis, University of Twente,, 2007.

[7] G. Cocorullo, et al., "Silicon Thermooptical Micromodulator with 700-kHz - 3-dB Bandwidth," IEEE Photonics Technology Letters, vol. 7, pp. 363-365, 1995.

[8] M.Kuramoto, "Chapter 3.5.1:Blue/Violet laser diodes for next generation DVD Application," in Wide bandgap semiconductors : fundamental properties and modern photonic and electronic devices, ed: Springer, 2007, pp. 97-230.

[9] H. M. H. Chong and R. M. D. L. Rue, "Tuning of photonic crystal waveguide microcavity by thermooptic effect," IEEE Photonics Technology Letters, vol. 16, pp. 1528-1530, 2004.

[10] R. Dekker, et al., "Ultrafast Kerr-induced all-optical wavelength conversion in silicon waveguides using $1.55 \mu \mathrm{m}$ femtosecond pulses," Optics Express, vol. 14, pp. 8336-8346, 2006.

[11] R. Soref and B. Bennett, "Electrooptical effects in silicon," IEEE Quantum Electronics, vol. 23, pp. 123 - 1291987.

[12] G. T. Reed and A. P. Knights, "Silicon Photonics: An Introduction," ed: Wiley, 2004, pp. 97-103.

[13] Q. Xu, et al., "Micrometre-scale silicon electro-optic modulator," Nature Letters, vol. 435, pp. 325-327, 2005.

[14] G. T. Reed and C. E. J. Png, "Silicon optical modulators," Materials today, vol. 8, pp. 4050, 2005.

[15] B. Maune, et al., "Liquid-crystal electric tuning of a photonic crystal laser," Applied Physics Letters, vol. 85, pp. 360-362, 2004.

[16] R. Karapinar, et al., "Polymer dispersed ferroelectric liquid crystal films with high electrooptic quality," Journal of Physics D: Applied Physics, vol. 35, pp. 900-905, 2002.

[17] L. J. Kauppinen, "Compact integrated optical devices for optical sensor and switching applications," PhD Thesis, University of Twente, 2010.

[18] S.Rajic, et al., "Feasibility of tunable MEMS photonic crystal devices," Ultramicroscopy, vol. 97, pp. 473-479, 2003.

[19] O. Levy, et al., "Mechanical tuning of two-dimensional photonic crystal cavity by micro electro mechanical flexures," Sensors and Actuators A: Physical, vol. 139, pp. 47-52, 2006. 
[20] W. Park and J. B. Lee, "Mechanically tunable photonic crystal structure," Applied Physics Letters, vol. 85, pp. 4845-4847, 2004.

[21] W. Park and J. B. Lee, "Mechanically tunable photonic crystals," Optics and Photonics News, vol. 20, pp. 40-45, 2009.

[22] C. Grillet, et al., "Reconfigurable photonic crystal circuits," Laser \& Photonics Reviews, vol. 4, pp. 192-204, 2010.

[23] G. Shambat, et al., "Tunable-wavelength second harmonic generation from GaP photonic crystal cavities coupled to fiber tapers," Optics Express, vol. 18, pp. 12176-12184, 2010.

[24] I. Märki, et al., "Tuning the resonance of a photonic crystal microcavity with an AFM probe," Optics Express, vol. 14, pp. 2969-2978, 2006.

[25] T. Takahata, et al., "Transmittance tuning of photonic crystal reflectors using an AFM cantilever," Sensors and Actuators A: Physical, vol. 128, pp. 197-201, 2006.

[26] W. C. L. Hopman, et al., "Nano-mechanical tuning and imaging of a photonic crystal micro-cavity resonance," Optics Express, vol. 14, pp. 8745-8752, 2006.

[27] P. T. Rakich, et al., "Ultrawide tuning of photonic microcavities via evanescent field perturbation," Optics Letters, vol. 31, pp. 1241-1243, 2006.

[28] B. Cluzell, et al., "A near-field actuated optical nanocavity," Optics Express, vol. 16, pp. 279-286, 2008.

[29] L. Lalouat, et al., "Near-field interactions between a subwavelength tip and a smallvolume photonic-crystal nanocavity," Physical Review B, vol. 76, pp. 041102-1-4, 2007.

[30] R. Dangel and W. Lukosz, "Electro-nanomechanically actuated integrated-optical interferometric intensity modulators and $2 \times 2$ space switches " Optics Communications, vol. 156, pp. 63-76, 1998.

[31] W. Lukosz, "Integrated optical nanomechanical devices as modulators, switches, and tunable frequency filters, and as acoustical sensors," in Proc. SPIE, Boston, MA, USA 1993, pp. 214-234.

[32] S. D. Senturia, "Chapter6:Energy-Conserving Transducers," in Microsystem Design, ed Massachusetts, United States: Kluwer Academic Publishers, 2003.

[33] G. J. Veldhuis, et al., "Electrostatically actuated mechanooptical waveguide ON-OFF switch showing high extinction at a low actuation-voltage," IEEE Journal of Selected Topics in Quantum Electronics, vol. 5, pp. 60-66, 1999.

[34] E. Bulgan, et al., "Submicron silicon waveguide optical switch driven by microelectromechanical actuator," Applied Physics Letters, vol. 92, pp. 101110-1-3, 2008.

[35] G. N. Nielson, et al., "Integrated wavelength-selective optical MEMS switching using ring resonator filters," IEEE Photonics Technology Letters, vol. 17, pp. 1190-1192, 2005.

[36] M. C. M. Lee, et al., "MEMS-actuated photonic crystal switches," IEEE Photonics Technology Letters, vol. 18, pp. 358-360, 2006.

[37] X. Chew, et al., "Dynamic tuning of an optical resonator through MEMS-driven coupled photonic crystal nanocavities," Optics Letters, vol. 35, pp. 2517-2519, 2010.

[38] X. Chew, et al., "An in-plane nano-mechanics approach to achieve reversible resonance control of photonic crystal nanocavities," Optics Express, vol. 18, pp. 22232-22244, 2010.

[39] W. Suh, et al., "Displacement-sensitive photonic crystal structures based on guided resonance in photonic crystal slabs," Applied Physics Letters, vol. 82, pp. 1999-2001, 2003.

[40] S. Iwamoto, et al., "Control of light propagation and localization in a photonic crystal slab by using a micromechanical actuator," Proceedings of the SPIE, vol. 5360, pp. 165-174, 2004.

[41] S. Iwamoto and Y. Arakawa, "Advances in photonic crystals with MEMS and with semiconductor quantum dots " Modern Trends in Laser Physics, vol. 16, pp. 223-231, 2006.

[42] Y. Kanamori, et al., "Control of guided resonance in a photonic crystal slab using microelectromechanical actuators," Applied Physics Letters, vol. 90, pp. 031911-1-3, 2007. 
Chapter 2. Mechano-optical modulators

[43] Y. Kanamori, et al., "Photonic crystal switch by inserting nano-crystal defects using MEMS actuator," in Proc. of. IEEE/LEOS International Conference on Optical MEMS, 2003, pp. 107-108.

[44] A. Higo, et al., "Development of high-yield fabrication technique for MEMS-PhC device," IEICE Electronics Express, vol. 3, pp. 39-43, 2006.

[45] S. Iwamoto, et al., "Observation of micromechanically controlled tuning of photonic crystal line-defect waveguide," Applied Physics Letters, vol. 88, p. 011104, 2006.

[46] K. I. Umemori, et al., "Photonic crystal waveguide switch with a microelectromechanical actuator," Applied Physics Letters, vol. 89, 2006.

[47] Y. Kanamori, et al., "An ultrasmall wavelength-selective channel drop switch using a nanomechanical photonic crystal nanocavity," Applied Physics Letters, vol. 95, pp. 1719111-3, 2009.

[48] W. Zhou, et al., "Novel reconfigurable semiconductor photonic crystal-MEMS device," Solid-State Electronics, vol. 50, pp. 908-913, 2006.

[49] T. Takahata, et al., "A wide wavelength range optical switch using a flexible photonic crystal waveguide and silicon rods," Journal of Micromechanics and Microengineering vol. 20, pp. 075009-1-6, 2010.

[50] S.Saravanan, et al., "A novel surface micromachining process to fabricate AlN unimorph suspensions and its application for RF resonators," Sensors and Actuators A: Physical, vol. 130-131, pp. 340-345, 2006. 


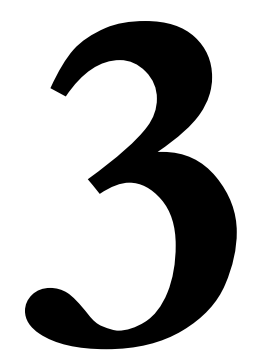

\section{Micro-bimorph cantilevers}

This chapter presents a systematic study on the modelling, fabrication and measurements of micro-bimorph cantilevers. The device, having stress induced upward curvature in the electrical offstate, functions as a vertical electrostatic actuator for nanometre displacements. A detailed analysis of the resonance frequency of the cantilever as a function of its length, deflection and thickness of the upper electrode layer, including the effect of undercut is carried out. A Galerkin based static model is developed to predict the pull-in voltages which are validated by measurements. A dynamic model is used to investigate the shift in resonance frequency by the electrostatic spring softening effect, which is evaluated against experimental data. The measured shift in resonance frequency is further extrapolated to non-destructively predict the pull-in voltages.

This chapter is submitted for publication as S.M.C. Abdulla, H. Yagubizade and G.J.M. Krijnen, "Analysis of resonance frequency and pull-in voltages of curled micro-bimorph cantilevers". 


\subsection{Introduction}

Micro-bimorph cantilevers constitute an important class of microelectromechanical systems (MEMS) having a wide range of application. As an actuator they are integrated with microscanners [1], microprobes [2], microelastic joints [3], microgrippers [4], atomic force microscopes [5], negative index materials [6], microvalves [7] and microrelays [8]. They are also used to sense environmental changes [9], to study thermal expansion coefficients [10] and to measure residual stress [11]. Routinely bimorph cantilevers are realised by coating a metal layer of higher thermal expansion coefficient on top of a dielectric layer. One way to excite bimorphs is by electrostatic actuation [12] where a voltage is applied between the upper metal electrode and the fixed substrate. Upon actuation, they can be operated in static or dynamic modes. The static mode exploits the variation in the deflection of the bimorph whereas the dynamic mode takes advantage of the changes in resonance frequency.

One of the results of metal- and dielectric material deposition is, the often undesired, curling of the bimorph due to either the stress gradient or the thermal missmatch induced stress in the different layers. This creates a variation in the gap along the length of the bimorph, e.g. having a small gap near the anchor point compared to that at the tip of the bimorph. On actuation, the electrostatic force generates a displacement of the tip of the bimorph. By utilising this effect, bimorph cantilevers can be integrated with either optical [13] or microwave [14] elements to serve as modulators, switches and wavelength tunable devices [15-16]. This chapter presents a detailed analysis on the static and dynamic behaviour of bimorph cantilevers to be used for modulation and tunability of integrated optical devices. Out of plane movement of the bimorph cantilevers in the evanescent field of various optical waveguiding structures like resonators and photonic crystals, can produce variations in modal propagation properties, i.e. changes in modal field shape, propagation constant or modal damping, allowing for mechano-optical modulation schemes [17]. Due to the limited distances over which the evanescent fields decay, only small displacements of mechanical members are required to interact profoundly with the propagating modes. These small distances on their turn allow for relative stiff cantilevers with resonance frequencies in the $\mathrm{MHz}$ range to interact with the optical element with distances typically less than $500 \mathrm{~nm}$. By optimising the thickness of various layers, the length and the voltage applied for the deflection, the frequency of vibration of the bimorph cantilever can be optimised for the required application.

Even though much research has been carried out in characterising and modelling various characteristics of micro-bimorph cantilevers, there are significant gaps remaining in understanding their properties such as the effect of curling on pull-in voltage and the 
dependence of resonance frequency on applied voltage. In characterizing electrostatic actuators, determination of the pull-in voltage $\left(\mathrm{U}_{\mathrm{p} i}\right)$ poses problems since most often after pull-in devices get stuck and have to be disregarded for further testing. Moreover determination of the pull-in voltage with high accuracy is tedious and time consuming since the quadratic nature of the dependence of electrostatic devices on actuation voltage requires measurements with small voltage increments. To make things even more complicated, pull-in under dynamic conditions will generally result in values lower than those found for quasi-static pull-in experiments. Early research [18-20] has used finite element method (FEM) simulations to study the pull-in instability in micro-cantilevers. Although powerful and helpful, it is less appropriate for providing a clear understanding of the physical characteristics of the device even after massive computations. References [21-23] considered the effect of curling of the beam by the stress gradient, developed models and compared it with measured data of Gupta [20]. The model developed by Saha et al. [18] has about 30\% error compared to measured values of Gupta [20]. The effects of fringing fields have been considered by $\mathrm{Hu}$ et al. [22], still arriving at analytical formulae. However, finally solving these equations, one proceeds most conveniently using a numerical iteration method. The model does not consider the elastic boundary effect of the anchor point but this effect has been included in a modified model in Chuang et al. [23]. Aforementioned studies are performed for static pull-in voltage calculation of monomorph cantilevers and their work does not extend to either the dynamic behaviour or to curled bimorph micro-cantilevers. Dynamic analysis of curled gold cantilevers is carried out by Som et al. [24] where residual stress is experimentally and theoretically studied from resonance frequency shift data. Although the measured pull-in voltages are close to their FEM analysis predictions, the linearized analytical solution predicts values twice as large as the measured ones.

This chapter presents a systematic investigation on the static and dynamic behaviour of micro-curled bimorph cantilevers, both analytically and experimentally. The effect of the upper electrode thickness on the off-state deflection, pull-in voltage and electrostatic spring softening effect is investigated. Moreover, the effect of undercut during the sacrificial layer etching which changes the length to its effective value is included in the model. For the analytic model, the position of the neutral axis, the stress in each layer and the stress induced moment of the structure are determined. A Galerkin based static model is developed to predict the pull-in voltage which is validated with the measured data. A dynamic model is developed to predict the frequency shift by the electrostatic spring softening (ESS) effect. This analytic model predicts the pull-in voltages and the shift in resonance frequencies with a relative mean error of about $6 \%$. In analogy with [25] where pull-in voltages of a clamped-clamped beams are predicted by 
extrapolating the plots of the measured frequency dependence on DC polarisation voltage, here this method is used to predict the pull-in voltage of curled micro-bimorphs. The method is analysed for bimorphs of varying length and upper electrode thicknesses and compared with actual pull-in measurements. This experimentally verifies a nondestructive method to accurately predict pull-in voltages, based on the ESS effect. Therefore, the theoretical and experimental studies carried out in this chapter gives considerable insight for designing micro-curled bimorph cantilevers.

The chapter is organised as described below. In the next section the models to predict the static and dynamic behaviour of the micro-curled bimorph cantilevers are presented. The design optimisation of micro cantilevers is presented in section 3.3. Section 3.4 describes the technology to fabricate the bimorphs. Section 3.5 deals with the various measurements carried out to study the bimorph. Analyses of the measurements and the model predictions are given in section 3.6. Finally, section 3.7 provides a discussion and 3.8 provide conclusions of the chapter.

\subsection{Model}

The bimorph structures studied here consist of two layers of dissimilar materials which deform (in the linearly elastic region) by the bimorph effect as well as by applied electrostatic forces. In this study a thin layer of chromium $(\mathrm{Cr})$ is used as upper electrode material. Since the bimorphs are being targeted for use in optical modulators through interaction with the evanescent field of guided modes, the dielectric layer is selected to be sufficiently thick in order to prevent optical loss. Approximately $1 \mu \mathrm{m}$ thick silicon-rich nitride $\left(\mathrm{SiN}_{x}\right)$ is selected as the dielectric material. The bimorphs are fabricated in a surface micromachining technology with silicon dioxide $\left(\mathrm{SiO}_{2}\right)$ as sacrificial layer.

\subsubsection{Static deflection}

In this section a detailed analysis is carried out to study the appropriate thickness of the $\mathrm{Cr}$ and $\mathrm{SiN}_{x}$ layers such that the first order mode resonance frequency of the bimorph is maximum for given minimum required off-state deflection (at zero voltage). To this end, an analytical expression for the resonance frequency of the first order mode is discussed first. Figure 3.1 (a) schematically shows the bimorph cantilever being analysed and (b) its coordinate systems. Table 3-I presents the material parameters and Table 3-II presents the geometrical parameters considered for the device. 

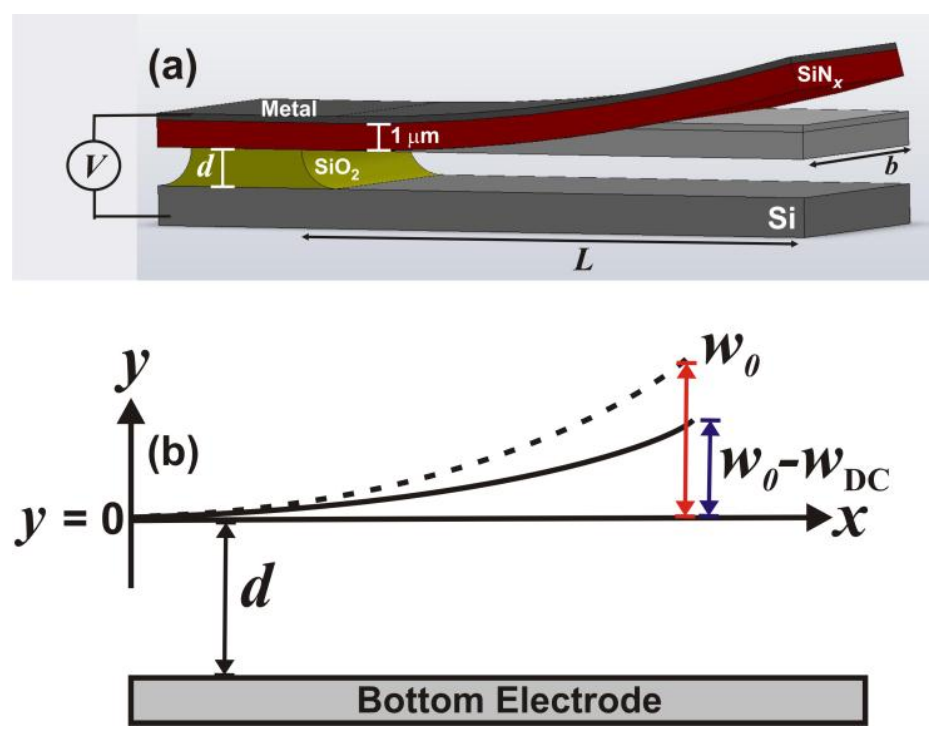

Figure 3.1. (a) Schematic illustration (Image source: E. Berenschot, TST Group, University of Twente, The Netherlands) and (b) the coordinate system of the micro-curled bimorph cantilever.

Table 3-I. The material parameters [26] of the micro-curled bimorph.

\begin{tabular}{|l|c|}
\hline \multicolumn{1}{|c|}{ Variables } & Values \\
Young's Modulus of $\mathrm{SiN}_{x}$ layer, $E_{\mathrm{SiN}_{x}}(\mathrm{MPa})$ & 276 \\
Young's Modulus of $\mathrm{Cr}$ layer, $E_{\mathrm{Cr}}(\mathrm{MPa})$ & 140 \\
Permittivity of free space, $\varepsilon_{0}(\mathrm{~F} / \mathrm{m})$ & $8.85 \times 10^{-12}$ \\
Relative dielectric constant for $\mathrm{SiN}_{\mathrm{x}}, \varepsilon_{\mathrm{SiN}_{x}}$ & 7.5 \\
\hline Density of SiN $\mathrm{x}$ layer, $\rho_{\mathrm{SiN}_{x}}\left(\mathrm{Kg} / \mathrm{m}^{3}\right)$ & 2887 \\
\hline Density of $\mathrm{Cr}$ layer, $\rho_{\mathrm{Cr}}\left(\mathrm{Kg} / \mathrm{m}^{3}\right)$ & 7190 \\
Thermal expansion coefficient for the $\mathrm{Cr}_{\text {layer, } \alpha_{\mathrm{Cr}}\left(\mathrm{K}^{-1}\right)}$ & $4.9 \times 10^{-6}$ \\
\hline Thermal expansion coefficient for the $\mathrm{SiN}_{\mathrm{x}}$ layer, $\alpha_{\mathrm{SiN}_{x}}\left(\mathrm{~K}^{-1}\right)$ & $2.8 \times 10^{-6}$ \\
\hline
\end{tabular}

Table 3-II. The geometrical parameters of the micro-curled bimorph.

\begin{tabular}{|c|c|}
\hline Variables & Values \\
Bimorph width, $b(\mu \mathrm{m})$ & 10 \\
Measured thickness of sacrificial layer, $d(\mu \mathrm{m})$ & 1.33 \\
Measured thickness of $\operatorname{SiN}_{x}$ layer, $t_{\mathrm{SiN}_{x}}(\mu \mathrm{m})$ & 1.09 \\
\hline
\end{tabular}

The position of the neutral axis after the deposition of the Cr layer is obtained by taking the condition that the resultant axial force acting on a cross section through the bimorph is zero. It is given relative to the lower side of the $\mathrm{SiN}_{x}$ layer as [27], 
Chapter 3. Micro-bimorph cantilevers

$$
y_{0}=\frac{E_{\mathrm{SiN}_{x}} t_{\mathrm{SiN}_{x}}^{2}+E_{\mathrm{Cr}_{\mathrm{Cr}}} t_{\mathrm{Cr}^{2}}^{2}+2 E_{\mathrm{Cr}} t_{\mathrm{SiN}_{x}} t_{\mathrm{Cr}}}{2\left(E_{\mathrm{SiN}_{x}} t_{\mathrm{SiN}_{x}}+E_{\mathrm{Cr}_{\mathrm{Cr}}} t_{\mathrm{Cr}}\right)}
$$

where $t_{\mathrm{Cr}}$ is the thickness of the $\mathrm{Cr}$ layer. After releasing, the $\mathrm{SiN}_{x}$ layer has a compressive stress with respect to the $\mathrm{Cr}$ layer. The tensile stress in the $\mathrm{Cr}$ layer tends to shorten while the compressive stress in lower $\mathrm{SiN}_{x}$ layer tends to elongate which eventually makes the bimorph curl upwards. For initial design purposes it is assumed that the bimorph is curved upward by thermal-mismatch induced stresses only. Hence any deposition and morphologically induced stresses are neglected in the model. The average thermal stress in each layer is given by

$$
\begin{gathered}
\sigma_{\mathrm{SiN}_{x}}=-E_{\mathrm{SiN}_{x}}\left(\frac{E_{\mathrm{Cr}} A_{\mathrm{Cr}}\left(\alpha_{\mathrm{Cr}}-\alpha_{\mathrm{SiN}_{x}}\right) \Delta T}{E_{\mathrm{Cr}} A_{\mathrm{Cr}}+E_{\mathrm{SiN}_{x}} A_{\mathrm{SiN}_{x}}}\right) \\
\sigma_{\mathrm{Cr}}=E_{\mathrm{Cr}}\left(\frac{E_{\mathrm{SiN}_{x}} A_{\mathrm{SiN}_{x}}\left(\alpha_{\mathrm{Cr}}-\alpha_{\mathrm{SiN}_{x}}\right) \Delta T}{E_{\mathrm{Cr}} A_{\mathrm{Cr}}+E_{\mathrm{SiN}_{x}} A_{\mathrm{SiN}_{x}}}\right)
\end{gathered}
$$

where $\Delta T\left(50^{\circ} \mathrm{C}\right)$ is the temperature difference between the $\mathrm{Cr}$ deposition temperature and room temperature, $A_{\mathrm{Cr}}$ is the cross sectional area of the $\mathrm{Cr}$ layer, $A_{\mathrm{SiN}_{x}}$ is the cross sectional area of the $\mathrm{SiN}_{x}$ layer respectively. The moment $(M)$ resulting from the stresses acting at the cross section is independent of $x$ and is given as,

$$
M=-b \sigma_{\mathrm{Cr}}\left(\frac{t_{\mathrm{Cr}}^{2}}{2}+t_{\mathrm{SiN}_{x}} t_{\mathrm{Cr}}-y_{0} t_{\mathrm{Cr}}\right)-b \sigma_{\mathrm{SiN}_{x}}\left(\frac{t_{\mathrm{SiN}_{x}}^{2}}{2}-y_{0} t_{\mathrm{SiN}_{x}}\right)
$$

This moment is counteracted by the bending moment in the bimorph. The (compound) flexural rigidity ( $E I)$ having the neutral axis at $y_{0}$ is

$$
\begin{aligned}
& E I=E_{\mathrm{SiN}_{x}} b\left(\frac{t_{\mathrm{SiN}_{x}}^{3}}{3}-y_{0} t_{\mathrm{SiN}_{x}}^{2}+y_{0}^{2} t_{\mathrm{SiN}_{x}}\right)+ \\
& E_{\mathrm{Cr}} b\left(\frac{t_{\mathrm{Cr}}^{3}}{3}+t_{\mathrm{SiN}_{x}} t_{\mathrm{Cr}}^{2}+t_{\mathrm{SiN}_{x}}^{2} t_{\mathrm{Cr}}-y_{0} t_{\mathrm{Cr}}^{2}+y_{0}^{2} t_{\mathrm{Cr}}-2 y_{0} t_{\mathrm{Cr}_{\mathrm{SiN}_{x}}}\right)
\end{aligned}
$$

In the differential equation of the static deflection curve of the beam, the boundary conditions [28] that at the clamped edge $(x=0)$ the deflection $w=0 ; w^{\prime}=0$ and at the free edge $(x=L) ; w^{\prime \prime}=0$ and $w^{\prime \prime \prime}=0$, are applied to get the deflection at the free end as,

$$
w(L)=\frac{M}{2 E I} L^{2}
$$

where $L$ is the length of the bimorph.

Thus, the radius of the initial stress induced curvature, for small deflection, can be approximated by, 


$$
R \approx \frac{L^{2}}{2 w_{L}}=\frac{E I}{M}
$$

where $w_{L}$ is the tip deflection of the bimorph.

\subsubsection{Mechanical resonance frequency}

This section analyses the dynamic behaviour of a micro-cantilever. An analytical expression for the resonance frequency of the micro-cantilever is derived solving the Euler-Bernoulli equation for the transverse free vibration of a beam [29],

$$
E I \frac{\partial^{4} w(x, t)}{\partial x^{4}}=-\rho A \frac{\partial^{2} w(x, t)}{\partial t^{2}} .
$$

Applying the boundary conditions [28] such that at the clamped edge $(x=0)$ the deflection $w=0 ; w^{\prime}=0$ and at the free edge $(x=L) ; w^{\prime \prime}=0$ and $w^{\prime \prime \prime}=0$, yield the characteristic equation for the structure as [24]

$$
\cos \left(k_{n} L\right) \cosh \left(k_{n} L\right)=-1
$$

where,

$$
k_{n}^{4}=\frac{\left(f_{n} / 2 \pi\right)^{2} \rho A}{E I} .
$$

In the above expression, $\rho A$ is the linear mass density, $f_{n}$ is the frequency of the $n^{\text {th }}$ order vibration mode and $w(x, t)$ is the deflection of the bimorph from its rest position. The normalised $n^{\text {th }}$ mode shape of the bimorph is,

$$
\Phi_{n}(x)=\left(\cosh \left(k_{n} x\right)-\cos \left(k_{n} x\right)\right)-\Theta\left(\sinh \left(k_{n} x\right)-\sin \left(k_{n} x\right)\right)
$$

Where

$$
\Theta=\frac{\sinh \left(k_{n} L\right)-\sin \left(k_{n} L\right)}{\cosh \left(k_{n} L\right)+\cos \left(k_{n} L\right)} ;
$$

$k_{\mathrm{n}}$ being satisfying equation (0.9) and $\Phi_{n}(x)$ satisfying equation [30],

$$
\int_{0}^{L} \Phi_{n}(x) \Phi_{m}(x) d x=\begin{aligned}
& 0 \text { for } m \neq n \\
& 1 \text { for } m=n
\end{aligned}
$$

From equation $(0.10)$ the $n^{\text {th }}$ mode resonance frequency at zero voltage is,

$$
f_{n}=\frac{1}{2 \pi}\left(\frac{C_{n}}{L}\right)^{2} \sqrt{\frac{E I}{b\left(t_{\mathrm{Cr}} \rho_{\mathrm{Cr}}+t_{\mathrm{SiN}_{x}} \rho_{\mathrm{SiN}_{x}}\right)}} .
$$

For the first mode $(n=1)$ of vibration, $k_{1} L=C_{1}=1.8751$ [31].

\subsubsection{Electro-mechanical coupling}

This section analyses static and dynamic behaviour of the electrostatically actuated curled micro-bimorph cantilevers. The electrostatic actuation voltage $(V)$ is given by a combination of a relatively large DC voltage $\left(V_{\mathrm{DC}}\right)$ and a relatively small AC voltage $\left(V_{\mathrm{AC}}\right)$,

$$
V=V_{\mathrm{DC}}+V_{\mathrm{AC}}(t)
$$


Hence the bimorph will have a deflection consisting of a static part $w_{0}(x)$ due to the stress-induced bending, a static part due to the DC term of the electrostatic force $w_{\mathrm{DC}}(x)$ and a time-dependent deflection denoted $u(x, t)$, given as

$$
w(x, t)=w_{0}(x)+w_{\mathrm{DC}}(x)+u(x, t)
$$

The effective dielectric gap between substrate and $\mathrm{Cr}$ electrode, including the dielectric contribution from the $\mathrm{SiN}_{x}$ layer, is given by

$$
g(x, t)=d+\left(t_{\mathrm{SiN}_{x}} / \varepsilon_{\mathrm{SiN}_{x}}\right)+w(x, t)=d^{\prime}+w(x, t)
$$

where $d^{\prime}=d+\left(t_{\mathrm{SiN}_{x}} / \varepsilon_{\mathrm{SiN}_{x}}\right)$. Assuming the Euler-Bernoulli beam model, the nonlinear equation of motion governing the dynamic behaviour of an electrostatic bimorph actuator including the fringing field effect [32] is,

$$
E I \frac{\partial^{4} w(x, t)}{\partial x^{4}}+\rho A \frac{\partial^{2} w(x, t)}{\partial t^{2}}=\frac{-\varepsilon_{0} b V^{2}}{2(g(x, t))^{2}}\left(1+0.65\left(\frac{g(x, t)}{b}\right)\right) .
$$

Next an analyze is carried out on the static and dynamic behaviour of the cantilever from equation (0.16).

\subsubsection{Static analysis to predict the pull-in voltage}

The pull-in phenomenon is one of the inherent instability situations for operating micro-cantilevers in a voltage controlled manner. It is crucial to accurately determine this instability point in order to properly design the sensitivity and dynamics of devices. The nonlinearity of the electrostatic force and the mechanical-electrical coupling effects complicate the accurate prediction of this instability point. Here the static behaviour of the bimorph under DC electrostatic actuation is analysed and a Galerkin based static model to predict the pull-in voltage is developed. The equation governing the DC induced static deflection is obtained from equation (0.16) by setting the time dependent terms (including the time dependent term in equation (0.14)) to zero;

$$
E I \frac{\partial^{4}\left(w_{0}+w_{\mathrm{DC}}\right)}{\partial x^{4}}=\frac{-\varepsilon_{0} b V_{\mathrm{DC}}^{2}}{2\left(d^{\prime}+w_{0}+w_{\mathrm{DC}}\right)^{2}}\left(1+0.65\left(\frac{d^{\prime}+w_{0}+w_{\mathrm{DC}}}{b}\right)\right)
$$

In order to linearize the above nonlinear static equation, the step by step linearization method (SSLM) developed by Rezazadeh et al. [33] is used. For this, it is considered that $w_{D C}^{i}$ is the displacement for the bimorph due to the application of the voltage $V_{\mathrm{DC}}^{i}$. A small increase in the applied voltage $\Delta V$ increases the displacement to [33]

$$
w_{\mathrm{DC}}^{i+1}=w_{\mathrm{DC}}^{i}+\psi(x) \text { when } V_{\mathrm{DC}}^{i+1}=V_{\mathrm{DC}}^{i}+\Delta V
$$

This converts equation (0.17) to the following equations,

$$
E I \frac{\partial^{4}\left(w_{0}+w_{\mathrm{DC}}^{i}\right)}{\partial x^{4}}=\frac{-\varepsilon_{0} b\left(V_{\mathrm{DC}}^{i}\right)^{2}}{2\left(d^{\prime}+w_{0}+w_{\mathrm{DC}}^{i}\right)^{2}}\left(1+0.65\left(\frac{d^{\prime}+w_{0}+w_{\mathrm{DC}}^{i}}{b}\right)\right)
$$




$$
E I \frac{\partial^{4}\left(w_{0}+w_{\mathrm{DC}}^{i+1}\right)}{\partial x^{4}}=\frac{-\varepsilon_{0} b\left(V_{\mathrm{DC}}^{i+1}\right)^{2}}{2\left(d^{\prime}+w_{0}+w_{\mathrm{DC}}^{i+1}\right)^{2}}\left(1+0.65\left(\frac{d^{\prime}+w_{0}+w_{\mathrm{DC}}^{i+1}}{b}\right)\right)
$$

Modifying equation (0.19) by substituting $w_{\mathrm{DC}}^{i+1}=w_{\mathrm{DC}}^{i}+\psi(x)$, subtracting equation (0.18) from it, doing a Taylor's expansion around $w_{\mathrm{DC}}^{i}$ and truncating it to the first order, the linearized equation to calculate $\psi(x)$ is obtained as

$$
\begin{aligned}
& E I \frac{\partial^{4} \psi}{\partial x^{4}}-\frac{\varepsilon_{0} b\left(V_{\mathrm{DC}}^{i+1}\right)^{2} \psi}{\left(d^{\prime}+w_{0}+w_{\mathrm{DC}}^{i}\right)^{3}}\left\{1+0.65\left(\frac{d^{\prime}+w_{0}+w_{\mathrm{DC}}^{i}}{2 b}\right)\right\} \\
& =\frac{-\varepsilon_{0} b\left(\left(V_{\mathrm{DC}}^{i+1}\right)^{2}-\left(V_{\mathrm{DC}}^{i}\right)^{2}\right)}{2\left(d^{\prime}+w_{0}+w_{\mathrm{DC}}^{i}\right)^{2}}\left\{1+0.65\left(\frac{d^{\prime}+w_{0}+w_{\mathrm{DC}}^{i}}{b}\right)\right\}
\end{aligned}
$$

Equation (0.20) is discretized (into 1000 nodes) and then solved to obtain $\psi$ at each step of the applied voltage. The applied voltage is incremented in steps of $0.1 \mathrm{~V}$. To this end $\psi(x)$ is approximated by a linear combination of eigen modes given by

$$
\psi(x)=\sum_{n=1}^{N} a_{n} \Phi_{n}(x)
$$

where $\Phi_{n}(x) \quad(n=1 \ldots . N)$ are the normalized mode shapes of a straight cantilever beam as given in equation (0.11). To improve the convergence, the Galerkin method [34] is used to specify the unknown constants for model amplitudes $a_{n}(n=1 \ldots . N)$. In this analysis, up to the third mode is included for calculating the pull-in voltage.

\subsubsection{Dynamic analysis to predict the ESS effect}

Many electrostatic actuators display a dynamic behaviour that includes easily observable resonances. The frequency of these resonances depends on ratios of mass and stiffness (or torsional stiffness and moment of inertia, or mass-density and [flexural] rigidity, etc.). Using voltage controlled actuation many of these structures have in common that they display an increase in electrical and decrease in effective stiffness often referred to as electrostatic spring softening (ESS) [25]. Due to ESS, the resonance frequency of electrostatic actuators can be effectively lowered on the application of a DC bias voltage. Additionally a small AC voltage added to the bias voltage can be used to excite the structures in order to measure the resonance frequencies. By linearizing equation (0.16) around the static deflection and neglecting the minor contribution of $u(x, t)$ to the fringing field correction (in practice $\left.u(x, t) / b \sim 10^{-3}\right)$ we get,

$$
\begin{gathered}
E I \frac{\partial^{4} w(x, t)}{\partial x^{4}}+\rho A \frac{\partial^{2} w(x, t)}{\partial t^{2}}=\frac{-\varepsilon_{0} b V^{2}}{2\left(d^{\prime}+w_{0}+w_{\mathrm{DC}}\right)^{2}}\left(1+0.65\left(\frac{d^{\prime}+w_{0}+w_{\mathrm{DC}}}{b}\right)\right)+ \\
\frac{\varepsilon_{0} b V^{2}}{\left(d^{\prime}+w_{0}+w_{\mathrm{DC}}\right)^{3}}\left(1+0.65\left(\frac{d^{\prime}+w_{0}+w_{\mathrm{DC}}}{2 b}\right)\right)(u(x, t))
\end{gathered}
$$


Chapter 3. Micro-bimorph cantilevers

In this analysis, the case where AC voltage is very less than the DC voltage $\left(V_{\mathrm{AC}}<<V_{\mathrm{DC}}\right)$ is considered to get the $V_{\mathrm{DC}}$ dependent eigen mode frequency. Substituting $(0.13)$ and $(0.14)$ into $(0.21)$ yields,

$$
\begin{aligned}
& E I \frac{\partial^{4}}{\partial x^{4}}\left(w_{0}+w_{\mathrm{DC}}+u(x, t)\right)+\rho A \frac{\partial^{2}}{\partial t^{2}}\left(w_{0}+w_{\mathrm{DC}}+u(x, t)\right) \\
& =\frac{-\varepsilon_{0} b V_{\mathrm{DC}}^{2}}{2\left(d^{\prime}+w_{0}+w_{\mathrm{DC}}\right)^{2}}\left(1+0.65\left(\frac{d^{\prime}+w_{0}+w_{\mathrm{DC}}}{b}\right)\right) \\
& +\frac{\varepsilon_{0} b V_{\mathrm{DC}}^{2}}{\left(d^{\prime}+w_{0}+w_{\mathrm{DC}}\right)^{3}}\left(1+0.65\left(\frac{d^{\prime}+w_{0}+w_{\mathrm{DC}}}{2 b}\right)\right)(u(x, t))
\end{aligned}
$$

Subtracting the stationary solution (0.17) from (0.22), gives

$$
E I \frac{\partial^{4} u(x, t)}{\partial x^{4}}+\rho A \frac{\partial^{2} u(x, t)}{\partial t^{2}}=\frac{\varepsilon_{0} b V_{\mathrm{DC}}^{2}}{\left(d^{\prime}+w_{0}+w_{\mathrm{DC}}\right)^{3}}\left(1+0.65\left(\frac{d^{\prime}+w_{0}+w_{\mathrm{DC}}}{2 b}\right)\right)(u(x, t))
$$

The solution of equation (0.23) is written by means of separation of variables $\left(A_{\mathrm{m}}\right.$ being the amplitude) as,

$$
u(x, t)=A_{\mathrm{m}} \Phi(x) \Psi(t)
$$

The first natural mode $\Phi(x)$ is taken as the assumed deflection shape function, which from equation $(0.11)$ is

$$
\Phi(x)=\cosh \left(k_{1} x\right)-\cos \left(k_{1} x\right)-\Theta\left(\sinh \left(k_{1} x\right)-\sin \left(k_{1} x\right)\right)
$$

where $k_{1} L=C_{1}=1.875$. Note that the method can be applied to higher modes as well by choosing $\Phi(x)$ accordingly. Substituting (0.24) into (0.23), multiplying the resulting equation by $\Phi(x)$ and integrating the outcome from 0 to $L$ yields

$$
\begin{aligned}
& E I \Psi(t)\left(\frac{C_{1}}{L}\right)^{4} \int_{0}^{L} A_{\mathrm{m}} \Phi^{2}(x) d x+\rho A \frac{\partial^{2} \Psi(t)}{\partial t^{2}} \int_{0}^{L} A_{\mathrm{m}} \Phi^{2}(x) d x= \\
& +\int_{0}^{L} \frac{\varepsilon_{0} b V_{\mathrm{DC}}^{2}}{\left(d^{\prime}+w_{0}+w_{\mathrm{DC}}\right)^{3}}\left(1+0.65\left(\frac{d^{\prime}+w_{0}+w_{\mathrm{DC}}}{2 b}\right)\right) A_{\mathrm{m}} \Psi(t) \Phi^{2}(x) d x
\end{aligned}
$$

The static solution obtained by solving equation (0.20) is substituted for $w_{\mathrm{DC}}$ in $(0.25)$ in order to determine the eigen values at given DC voltages. Comparing equation (0.25) with the general differential force $(F)$ equation for an object of mass ' $m$ ' and spring constant ' $K$ ', experiencing single harmonic motion, $m\left(\partial^{2} \Psi / \partial t^{2}\right)+K \Psi=F$, the first mode resonance frequency is obtained as,

$$
f_{1}=\frac{1}{2 \pi} \sqrt{\frac{E I\left(\frac{C_{1}}{L}\right)^{4} \int_{0}^{L} \Phi^{2}(x) d x-K^{\text {electrical }}}{\rho A \int_{0}^{L} \Phi^{2}(x) d x}}
$$

Where 


$$
K^{\text {electrical }}=\int_{0}^{L}\left[\frac{\varepsilon_{0} b V_{\mathrm{DC}}^{2}}{\left(d^{\prime}+w_{0}+w_{\mathrm{DC}}\right)^{3}}\left(1+0.65 \frac{\left(d^{\prime}+w_{0}+w_{\mathrm{DC}}\right)}{2 b}\right)\right] \Phi^{2}(x) d x
$$

Equation (0.26) is of the form

$$
f_{1}=\frac{1}{2 \pi} \sqrt{\frac{K^{\text {mechanical }}-K^{\text {electrical }}}{M}} .
$$

When the DC bias voltage term is zero, the term $K^{\text {electrical }}$ tends to zero which yields the simple analytical expression for the resonance frequency of the bimorph as in equation (0.12). Hence, the change of resonance frequency by the applied voltage (ESS) is retained in the term $K^{\text {electrical }}$.

\subsection{Optimisation}

The design is optimised taking $t_{\mathrm{Cr}}$ as a variable. Thickness of $\mathrm{SiN}_{x}$ is fixed at a value of $1.0 \mu \mathrm{m}$. The off-state displacement $\left(w_{L}\right)$ was chosen as an input parameter and the designs were evaluated for $w_{L}<300 \mathrm{~nm}$. Hence, six values of $w_{L}$ is considered from $50 \mathrm{~nm}$ to $300 \mathrm{~nm}$ in steps of $50 \mathrm{~nm}$. The approach is graphically illustrated in Figure 3.2.

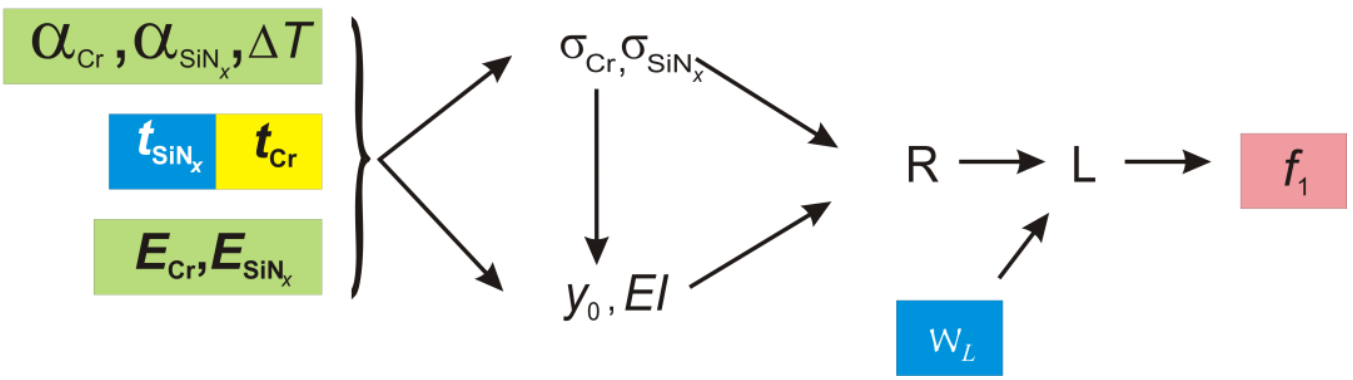

Figure 3.2. Calculation strategy: Green boxed values are fixed material properties. Blue boxed values were chosen. Cr thickness was varied to find an optimum design.

Using the material parameters, layer thicknesses and equations (0.2) and(0.3), the stresses $\sigma_{\mathrm{Cr}}$ and $\sigma_{\mathrm{SiN}_{x}}$ in the bimorph cantilever are calculated. From the same input plus the stress, the neutral axis (0.1) and the flexural rigidity (0.5) are calculated. From these results, the radius of curvature is determined according to (0.7). Making use of (0.6) and (0.7) and taking a required off-state deflection as input, the bimorph length is determined. Finally, from (0.12) the resonance frequency is derived as functions of $t_{\mathrm{Cr}}$. Starting from $t_{\mathrm{Cr}}=0$, there is an increasing stress in the bimorph. However, the flexural rigidity is not significantly changing up to $\mathrm{Cr}$ thickness comparable to the $\mathrm{SiN}_{x}$ thickness. Hence, for $t_{\mathrm{Cr}}$ much smaller than $t_{\mathrm{SiN}_{x}}$, the radius of curvature is almost proportional to $1 / t_{\mathrm{Cr}}$ (see Figure 3.3). When the $\mathrm{Cr}$ thickness is comparable to the $\mathrm{SiN}_{x}$ thickness, the stress induced moment becomes maximum and hence the radius of curvature is minimum at about 2 $\mathrm{mm}$. Here, the shortest required bimorph lengths are obtained as well. 
Chapter 3. Micro-bimorph cantilevers

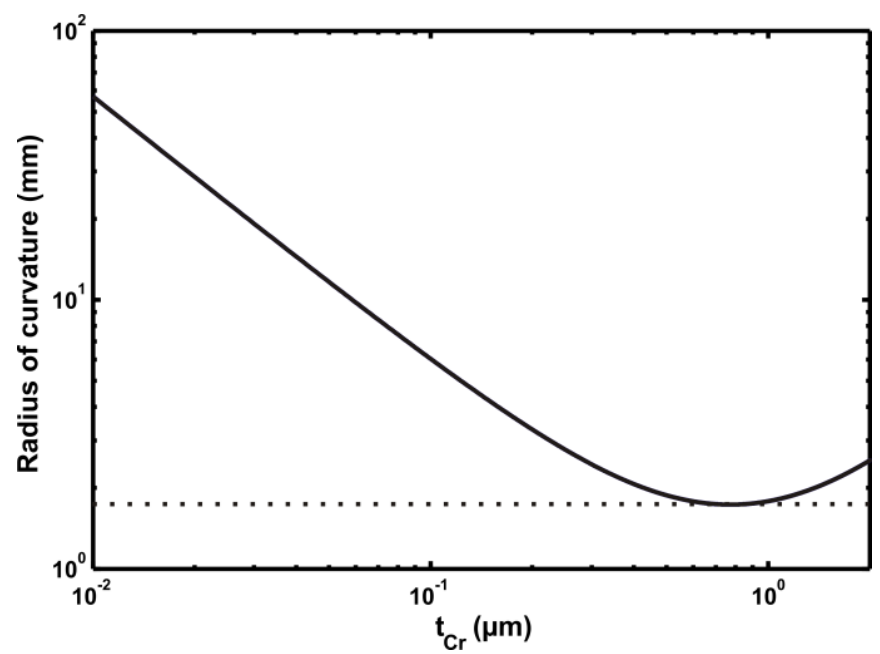

Figure 3.3. Radius of the stress induced curvature of the bimorph as a function of the Cr thickness.

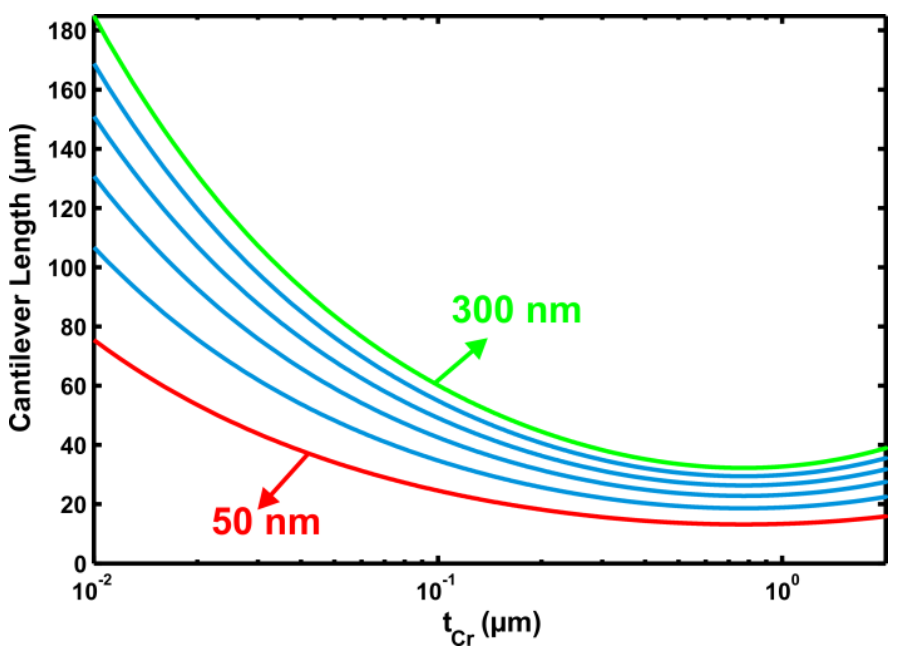

Figure 3.4. Required length of the bimorph as a function of the Cr thickness for $w_{\mathrm{L}}=50 \mathrm{~nm}$ to $w_{\mathrm{L}}=300 \mathrm{~nm}$.

The length of the bimorph structure not only depends on the radius of curvature but also on the requirements of the off-state upward deflection $w_{\mathrm{L}}$. Figure 3.4 shows the required length as a function of $t_{\mathrm{Cr}}$ with $w_{\mathrm{L}}$ as the parameter. Clearly, for larger $w_{\mathrm{L}}$ the required bimorph length increases, but this increase is modest since the displacement depends quadratically on the length (equation(0.6)). Minimum required length of the bimorph structures is obtained between 800 and $900 \mathrm{~nm} \mathrm{Cr}$ thicknesses. At very thin $\mathrm{Cr}$ layer thickness, the radius of curvature is large and hence the required bimorph length to get a set off-state deflection becomes large as well. However, if the length of the cantilever is increased beyond a certain length then during the release of the bimorph using wet sacrificial etching, the cantilever will have the tendency to stick to the substrate and stay there [35]. So, the length of the cantilever has to be less than its critical length of sticking. Using dry or vapour etching methods, these restrictions may be relaxed. 


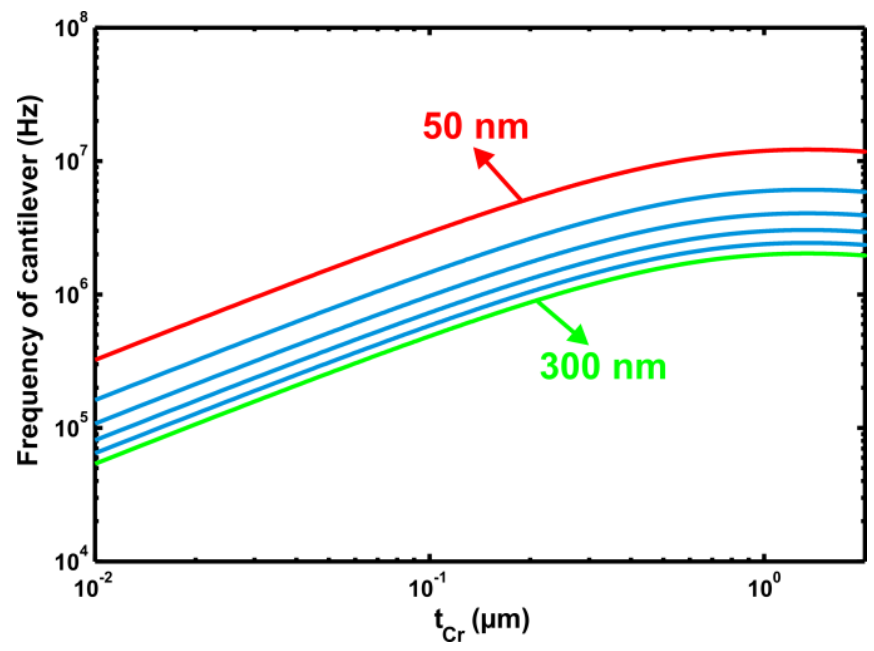

Figure 3.5. Achievable first order mode resonance frequency as a function of the Cr thickness for $w_{L}=50$ $n m$ to $w_{L}=300 \mathrm{~nm}$.

In Figure 3.5, the frequency of the cantilever in its first mode of vibration is plotted as a function of the thickness of the Cr layer, keeping the thickness of $\operatorname{SiN}_{x}$ as 1.0 $\mu \mathrm{m}$. The plots are for different off-state deflections ranging from $50 \mathrm{~nm}$ to $300 \mathrm{~nm}$. It is seen that the frequency of vibration is high for a lower deflection and vice versa. As the thickness of $\mathrm{Cr}$ increases up to about $1 \mu \mathrm{m}$, the frequency of vibration increases where the cantilever length is a minimum. Figure 5 shows that the resonance frequencies as high as $10 \mathrm{MHz}$ are feasible for $50 \mathrm{~nm}$ off-state deflection, dropping to about $1 \mathrm{MHz}$ for 300 nm off-state deflection. However it has to be noted that high resonance frequencies come at the price of large switching voltage.

\subsection{Fabrication}

The bimorph cantilevers are fabricated by surface micromachining techniques on a $100 \mathrm{~mm}$ diameter $\mathrm{p}$-type silicon wafer (orientation $<100>$ ). The silicon wafer has a thickness of $525 \mu \mathrm{m}( \pm 25 \mu \mathrm{m})$ and a resistivity of $5-10 \Omega \mathrm{cm}$. The fabrication process is a simple one mask process, using a positive photoresist (Olin 907-17) for the UV lithography. The patterned top electrode layer also acts as a masking layer for etching of the $1.0 \mu \mathrm{m}$ thick dielectric layer. Timed etching of the sacrificial layer releases the cantilevers and defines the anchor point $(300 \times 300 \mu \mathrm{m})$ for the bond pads of the bimorph. The cantilever design length is varied from $20 \mu \mathrm{m}$ to $100 \mu \mathrm{m}$ and the width is fixed at $10 \mu \mathrm{m}$ in all cases. The fabrication flow for the device is shown in Figure 3.6. 
Chapter 3. Micro-bimorph cantilevers

(a)

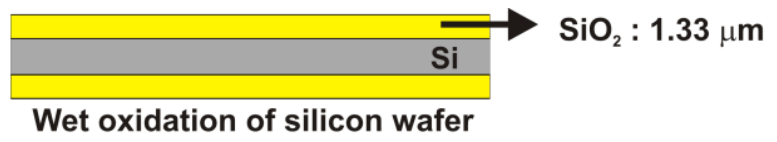

(b)

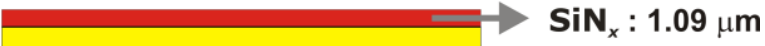

(c)

Deposition of $\mathrm{SiN}_{x}$ layer

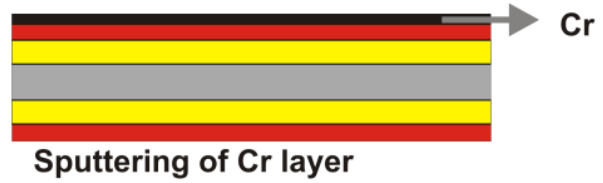

(d)

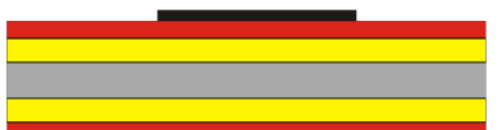

Patterning and wet etching of $\mathrm{Cr}$ layer

(e)

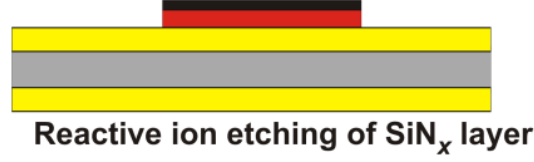

(f)

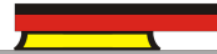

Sacrificial layer etching

Figure 3.6. The fabrication flow for the micro-bimorph cantilever.

The silicon wafers are (a) first oxidized at $1150^{\circ} \mathrm{C}$ by wet oxidation to a (measured) thickness of $1.33 \mu \mathrm{m}$. Next (b) a layer of low stress $\operatorname{SiN}_{x}$ is deposited by low pressure chemical vapour deposition (LPCVD) [36] for a (measured) thickness of $1.09 \mu \mathrm{m}$. (c) The upper $\mathrm{Cr}$ layer is sputtered with varying thicknesses whose measured values are $59 \mathrm{~nm}, 97$ $\mathrm{nm}, 145 \mathrm{~nm}, 204 \mathrm{~nm}$ respectively. (d) The Cr layer is patterned using the mask Olin 90735 (thickness of resist $=3.5 \mu \mathrm{m}$ ). Followed by this, $\mathrm{Cr}$ is wet etched in chromium etchant (MERCK 111547.2500). Next, (e) the $\mathrm{SiN}_{x}$ layer is etched from front and back sides by reactive ion etching (RIE, Elektrotech PF 340, at $10^{\circ} \mathrm{C}, 75 \mathrm{~W}$ power, $10 \mathrm{~m}$ Torr pressure) using a $\mathrm{CHF}_{3} / \mathrm{O}_{2}(25 \mathrm{sccm} / 5 \mathrm{sccm})$ gas mixture. After this, the resist is stripped in $\mathrm{HNO}_{3}(99 \%)$ for 20 minutes. Finally (f) the sacrificial silicon oxide layer is etched in buffered hydrofluoric acid $\left(\mathrm{BHF}, \mathrm{NH}_{4} \mathrm{~F}: \mathrm{HF}=7: 1\right)$ followed by the freeze drying release step [37] (See also Appendix I). A detailed fabrication flow of this process is presented in Appendix II-Table I. A scanning electron microscope (SEM) image of a fabricated cantilever showing an upward curvature is shown in Figure 3.7. 


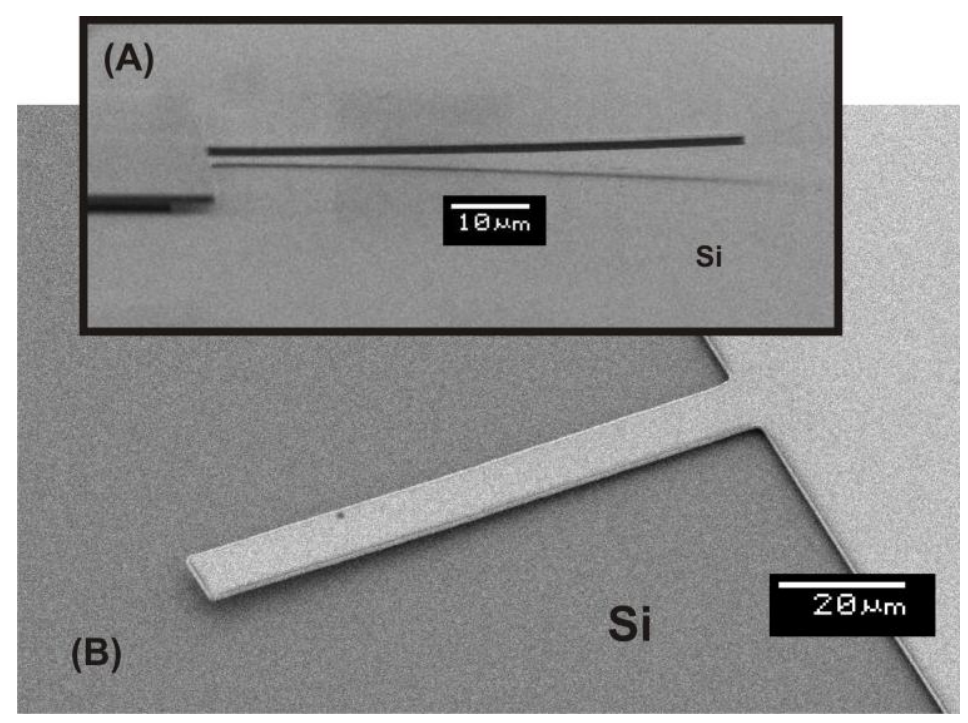

Figure 3.7. SEM images showing different views of the micro-cantilevers clearly showing the upward curvature.

\subsection{Measurements}

In order to characterise the system and its performance, various experiments were carried out during the fabrication and after releasing the bimorphs. All the measurements described in this chapter were carried out at room temperature and at atmospheric pressure. The exact thickness of each layer deposited was measured using ellipsometery, using dummy wafers. Figure 3.8 shows the SEM image of the bondpad of the cantilever clearly showing the undercut introduced during sacrificial layer etching. Since the cantilevers with varying $\mathrm{Cr}$ thicknesses were realised on various wafers, which were individually processed in the SLE step, their undercut was slightly varying as well. The undercut as measured with an optical microscope, for various electrode thicknesses are given in Table 3-III.

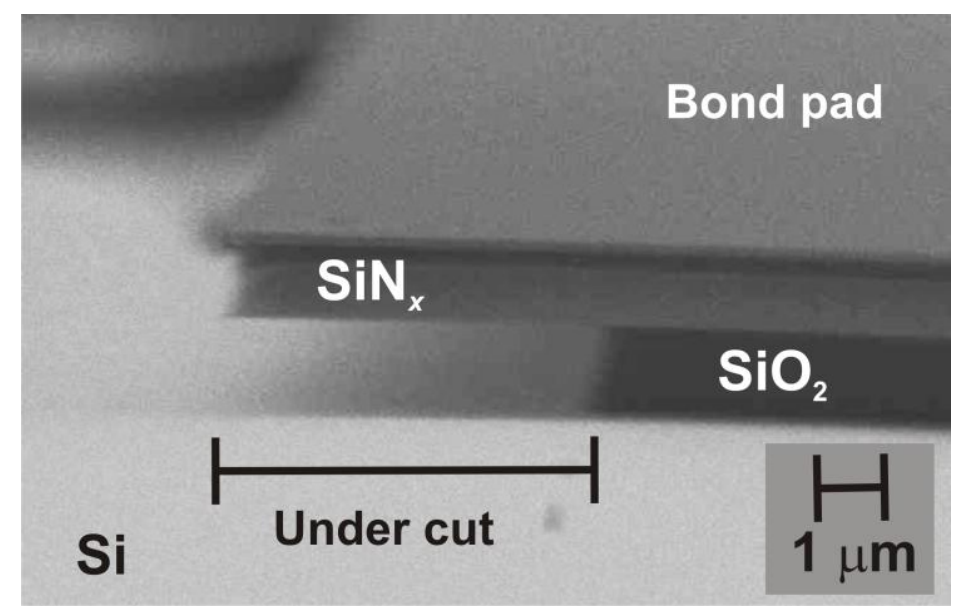

Figure 3.8. SEM image showing undercut in the bondpad of a micro-cantilever. 
Table 3-III. Measured undercut for various Cr layer thicknesses.

\begin{tabular}{|c|c|}
\hline$t_{C r}(\mathrm{~nm})$ & Measured undercut $(\mu \mathrm{m})$ \\
97 & 5.6 \\
145 & 5.5 \\
204 & 5.4 \\
\hline
\end{tabular}

\subsubsection{Residual stress}

The stress in the sputtered Cr layer is an important parameter, which causes the off-state deflection of the bimorph making it of importance to monitor it. Before and after sputtering of the Cr layers, wafer curvature measurements were carried out over the wafer diameter through 0 and 90 degrees using a Dektak-V.8 surface profiler. From the mean deflection of these layers the stress in the layer is calculated from Stoney's formula [38],

$$
\sigma_{0}=\frac{4}{3} \frac{E^{\prime}}{(1-v)} \frac{t^{2} v}{d_{\text {thin }} D^{2}}
$$

where $\sigma_{0}$ is the residual film stress, $E^{\prime}$ is the Young's modulus of the substrate material, $t$ is the thickness of the wafer, $d_{\text {thin }}$ is the thickness of the thin film deposited, $v$ is the Poisson's ratio of the substrate material, $v$ is the deflection at the centre of the surface scan and $D$ is the scan length over the wafer. Table $3-I V$ shows the calculated stress values for various $\mathrm{Cr}$ layer thicknesses from the wafer curvature measurements.

Table 3-IV. Calculated stress values for various Cr layers from the wafer curvature measurements.

\begin{tabular}{|c|c|}
\hline$t_{C r}(\mathrm{~nm})$ & Mean stress (MPa) \\
59 & 621.7 \\
97 & 868.8 \\
145 & 371.5 \\
204 & 314.2 \\
\hline
\end{tabular}

\subsubsection{Off-state deflection}

The off-state deflection of the bimorphs is measured by white light interference microscopy (WLIM, Polytec MSA400) and a typical measurement is as shown in Figure 3.9. The off-state tip deflection is measured for various lengths and $\mathrm{Cr}$ thicknesses as shown in Figure 3.10. As expected the tip deflection increase as a function of the length and the $\mathrm{Cr}$ layer thickness. However the measured curvature is much more than anticipated. 


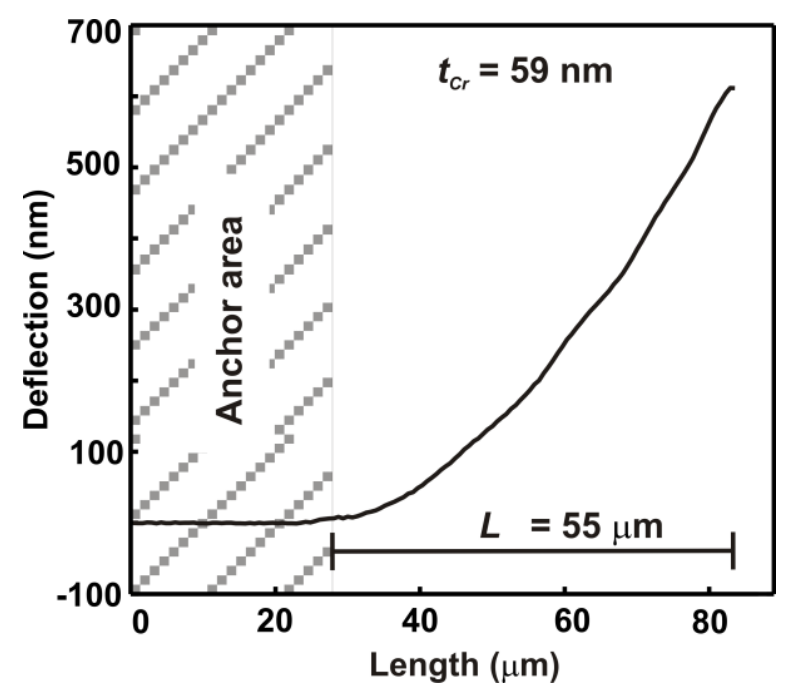

Figure 3.9. White light interferometry measurement showing the off-state upward deflection of a $55 \mu \mathrm{m}$ long cantilever with $59 \mathrm{~nm}$ thick Cr layer.

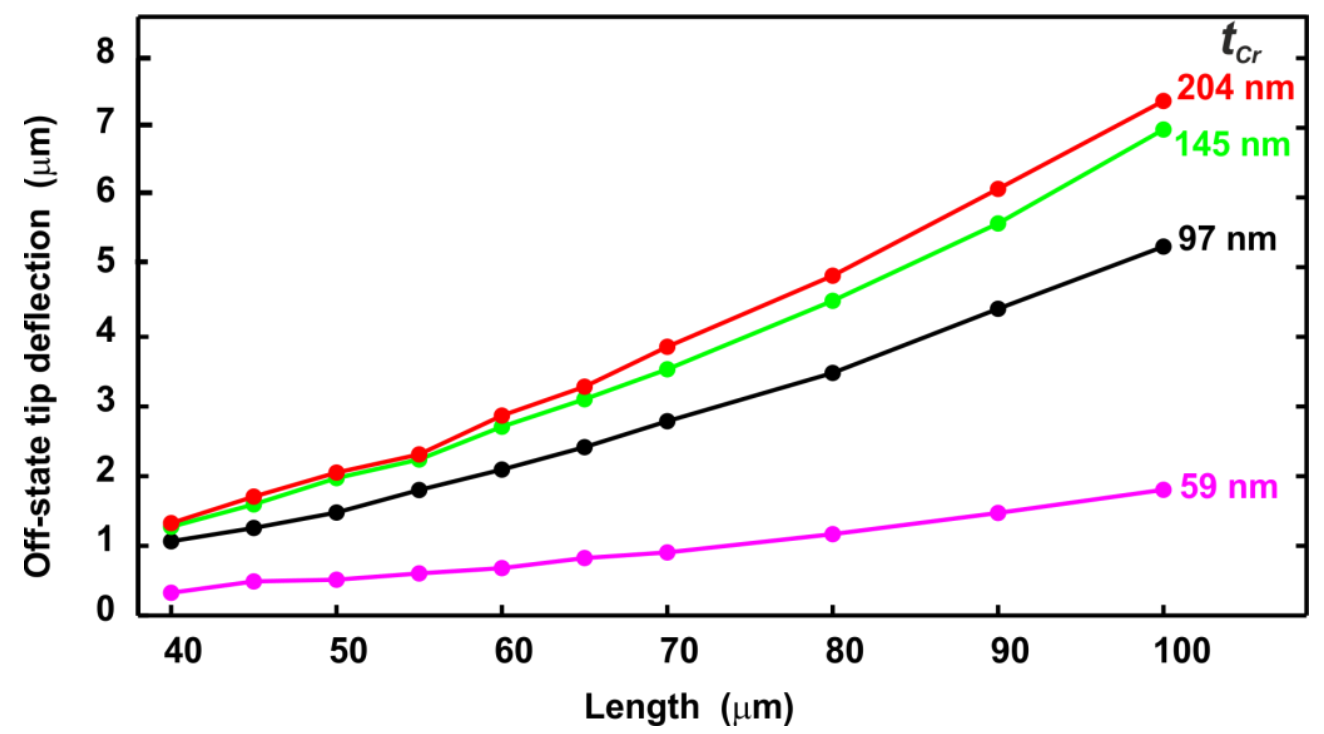

Figure 3.10. Off-state tip deflections of bimorphs of various lengths and $\mathrm{Cr}$ layer thicknesses, measured by WLIM.

\subsubsection{Resonance frequency}

The resonance frequency is analysed as a function of the length for various $\mathrm{Cr}$ layer thicknesses. Measurements on resonance frequency, amplitude of vibration, phase shift, quality factor and pull-in voltage are carried out by a Laser Doppler Vibrometer (LDV, Polytec MSA400). A typical measurement from the LDV showing the first three resonant modes and the corresponding phase shifts is shown in Figure 3.11. 
Chapter 3. Micro-bimorph cantilevers

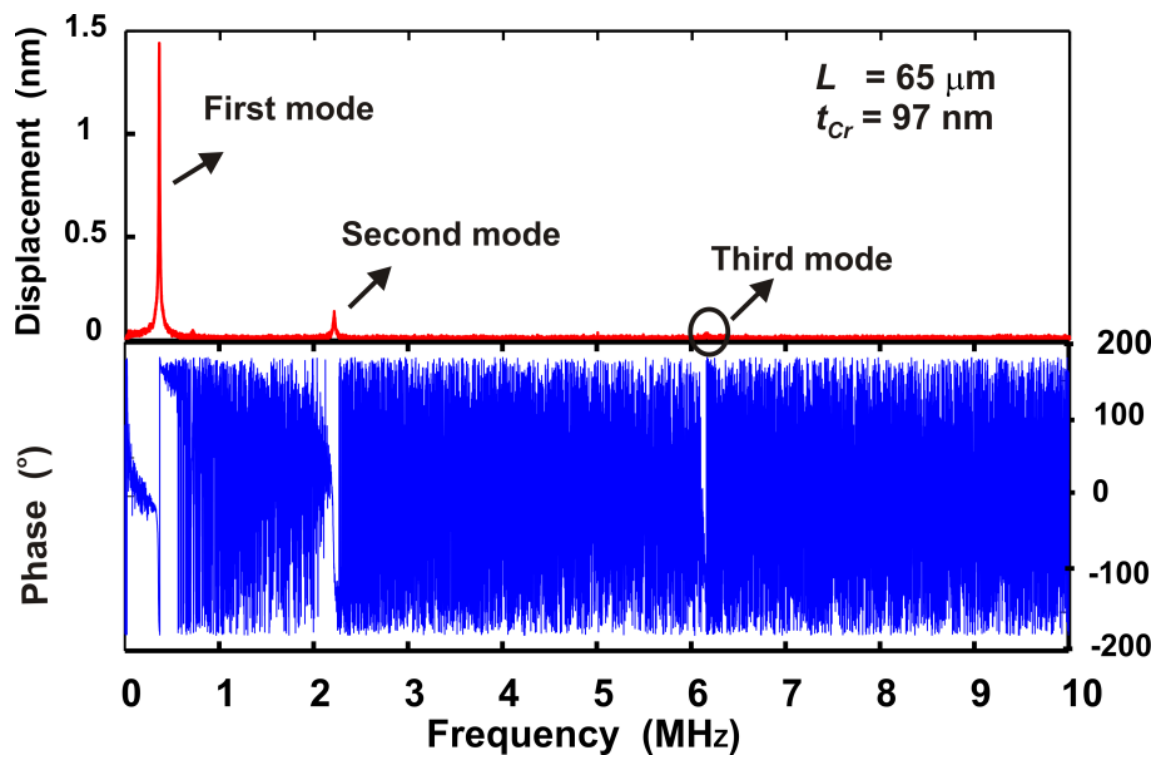

Figure 3.11. Frequency response as measured by $L D V$ showing the first three resonance frequencies and corresponding phase shifts, for a $65 \mu \mathrm{m}$ long bimorph.

\subsubsection{Electro static spring softening effect}

The shifts in resonance frequency due to the application of a DC voltage were measured for bimorph lengths varying from $40 \mu \mathrm{m}$ to $100 \mu \mathrm{m}$. For these measurements $V_{\text {DC }}$ is varied from $5 \mathrm{~V}$ to near the pull-in values. A small AC voltage of $0.5 \mathrm{~V}$ was applied to excite the structures in order to measure the resonance frequencies. These measurements were used to extrapolate the resonance frequency to $0 \mathrm{~V}\left(f_{0}\right)$ and the pullin voltages.

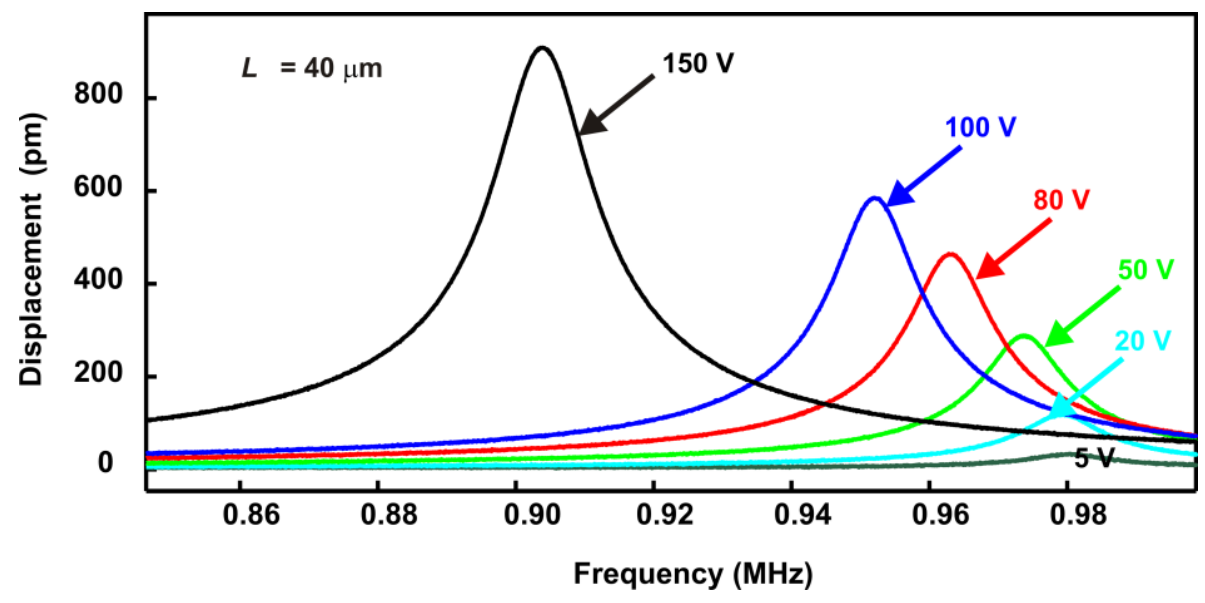

Figure 3.12. Measured first mode resonance frequency shift due to ESS effect for various $V_{\mathrm{DC}}$.

The resonance frequency shift for a bimorph of design length of $40 \mu \mathrm{m}$ at different $V_{\mathrm{DC}}(5 \mathrm{~V}, 20 \mathrm{~V}, 80 \mathrm{~V}, 100 \mathrm{~V}$ and $150 \mathrm{~V})$, measured directly from the LDV is shown in Figure 3.12. It is clearly observed that an increase in DC bias voltage increases the amplitude of vibration but decreases the resonance frequency. Figure 3.13 shows the 
normalised first order mode resonance frequency versus normalised DC voltage for various bimorph lengths and for the two Cr layer thicknesses. It is observed that the normalised curves for all these bimorphs virtually coincide.

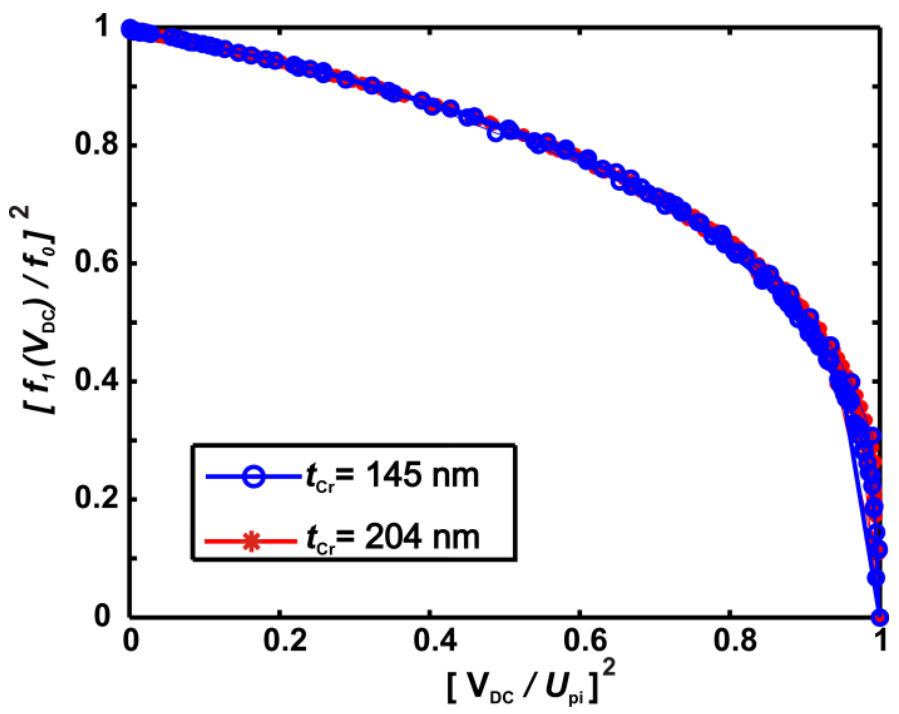

Figure 3.13. Normalised first mode resonance frequency versus normalised polarisation voltage for various bimorph lengths of two different $\mathrm{Cr}$ thicknesses.

In order to investigate the effect of possibly trapped charges in the $\mathrm{SiN}_{x}$ dielectric layer, the resonance frequencies were also measured by applying a negative bias voltage between the two electrodes. Figure 3.14 shows the results of positive and negative bias voltages on the first order mode resonance of a bimorph. From the symmetric shape of the graph it is concluded that the effect of charge trapping is negligibly small for this cantilever.

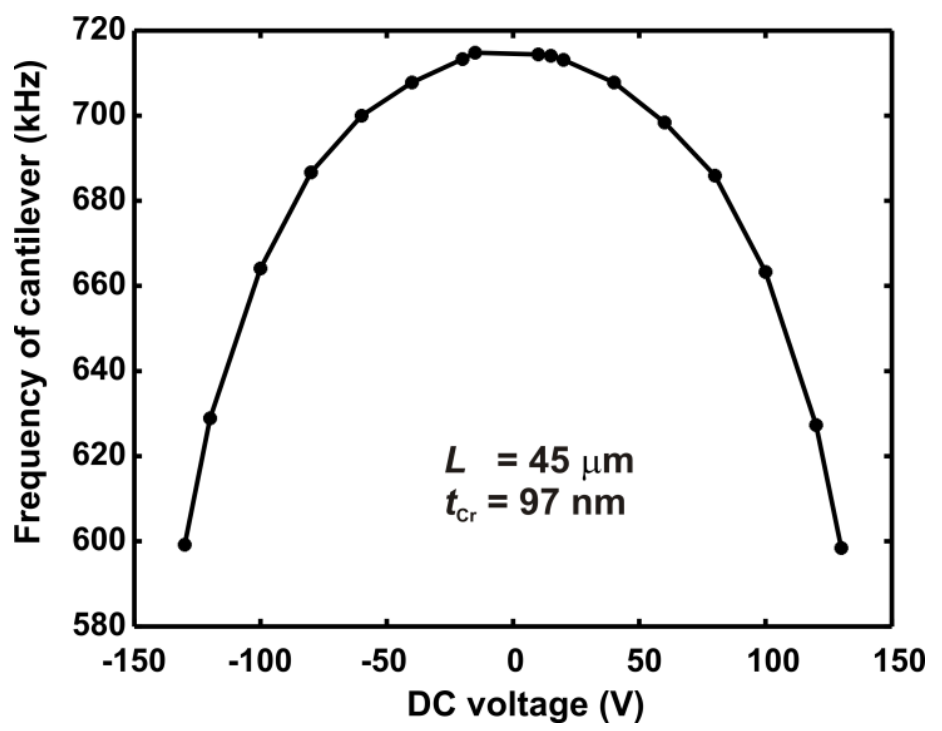

Figure 3.14. Effect of positive and negative bias voltages on the first mode resonance frequency of a bimorph. 
Chapter 3. Micro-bimorph cantilevers

\subsection{Analysis}

\subsubsection{Resonance Frequency}

The first and second resonance frequency measured for various lengths of the bimorphs are compared with predictions of equation (0.12). It is found that the predicted values show a deviation in resonance frequency especially for cantilevers of shorter length. This holds for both the first and second order modes of resonance. This is associated with the undercut of the bond pad during the SLE, which causes an increase in effective length of the cantilever compared to designed values. This effective length and the effective flexural rigidity of the cantilever in turn cause a deviation in the resonance frequency that is a function of $L$ and $E I$. To account for this effect from equation $(0.12)$ we have,

$$
\frac{\Delta f_{1}}{f_{1}}=-2 \Delta L\left(\frac{1}{L}\right)+\left(\frac{\Delta E I}{2 E I}\right)
$$

where $\Delta f_{1}$ is the difference between the experimental and predicted values of resonance frequencies, $\Delta L$ is the effective deviation in length and $\Delta E I$ is the effective deviation in flexural rigidity of the cantilever. Ideally equation (0.28) describes a straight line for $\left(\Delta f_{1} / f_{1}\right)$ versus $L^{-1}$ with slope $-2 \Delta L$ and with value $\Delta E I / 2 E I$ as the ordinate for $L^{-1}=0$. This implies that both effects can be determined independently. Using a least square method $\Delta L$ and $\Delta E I / E I$ are determined. After including these effects, model predictions of the resonance frequencies match well with the measured values. The analysis is performed for both the first and second order resonance frequencies and for three Cr layer thicknesses. The results are shown in Figure 3.15-Figure 3.17.

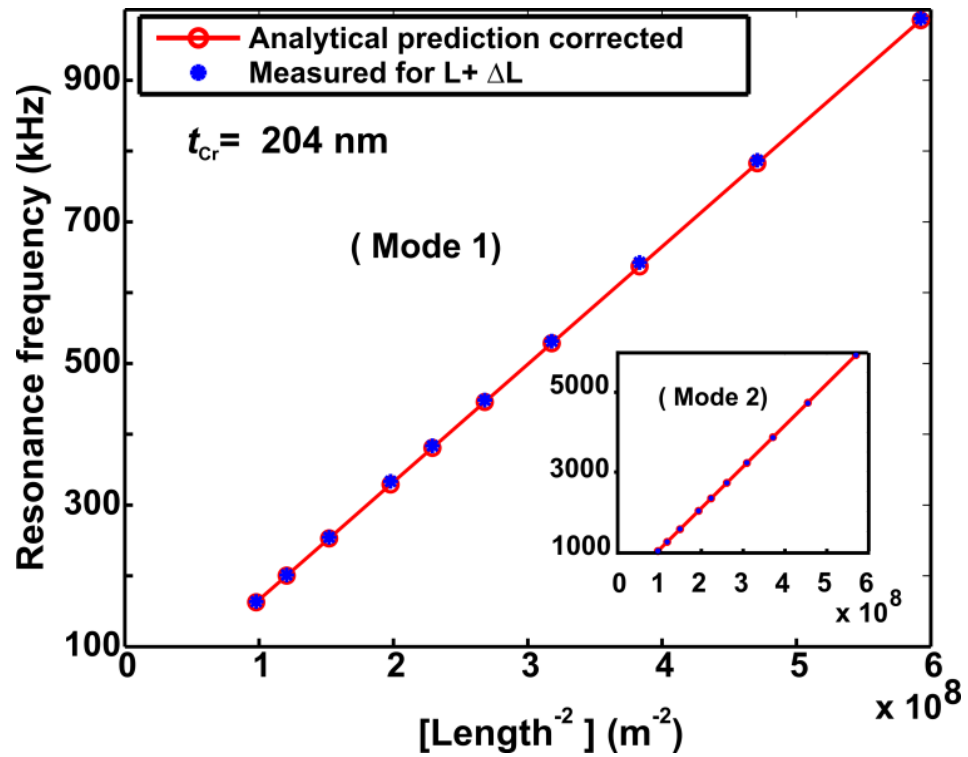

Figure 3.15. First and second (inset) order mode resonance frequency versus the corrected length of the bimorphs for an electrode thickness of $204 \mathrm{~nm}$. 
Figure 3.15-Figure 3.17 show that the resonance frequencies for all the bimorphs can be fairly well predicted as a function of length when an effective length is considered that takes into account the added flexibility due to the undercut at the anchor point. For the thicker $\mathrm{Cr}$ layers the effective addition to the design length decreases which can be understood from an increased stiffness of the layer-stack. However, for different modes different effective additional lengths are found; higher order modes showing larger effective additional length. Although this makes the method less convenient from a design-point of view it can be understood from the larger deflections amplitudes of the higher order modes near the anchor points. As such it is to be expected that the method can be extended to predict effective additional lengths in dependence of the order of the mode of vibration. Alternatively one may attempt to calculate the finite stiffness of the anchors and incorporate this in an elastic boundary based modal analysis [39].

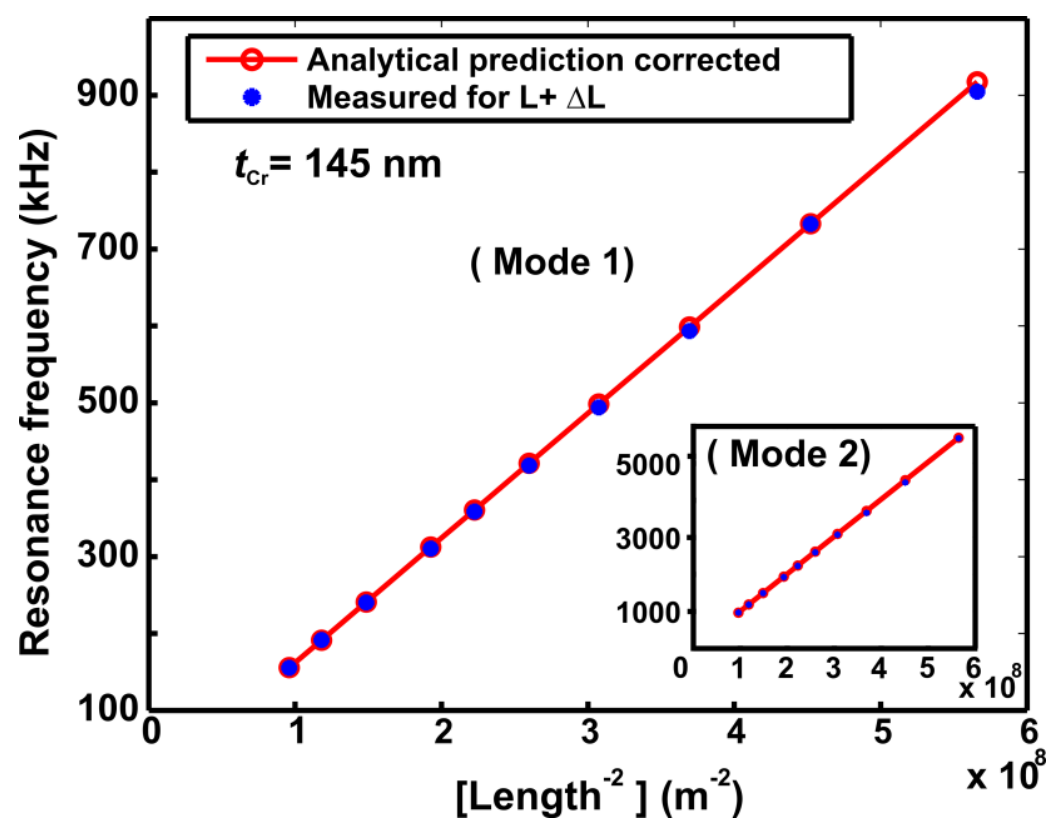

Figure 3.16. As Figure 3.15 for Cr thickness of $145 \mathrm{~nm}$. 
Chapter 3. Micro-bimorph cantilevers

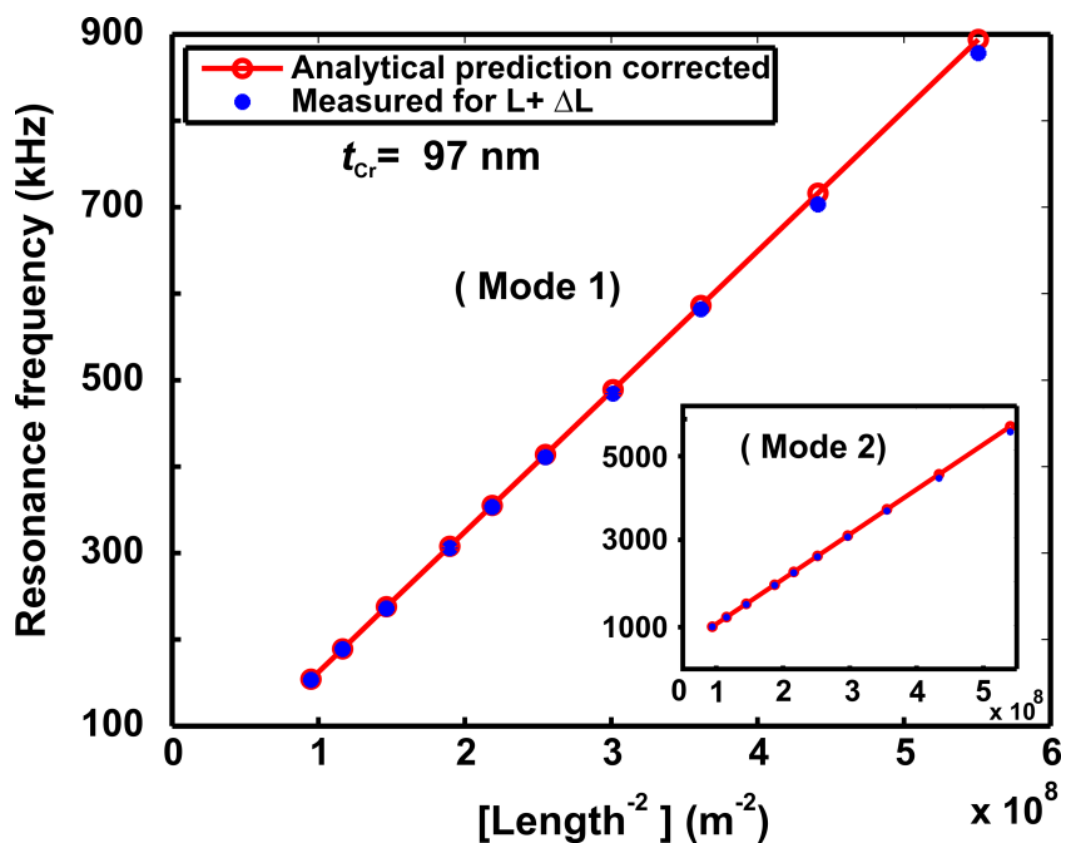

Figure 3.17. As Figure 3.15 for Cr thickness of $97 \mathrm{~nm}$.

Table $3-\mathrm{V}$ shows the calculated values for $\Delta L$ and $E I_{\text {corrected }}$ for different $\mathrm{Cr}$ layer thicknesses for first and second order flexural modes.

Table 3-V. Effect of undercut on different Cr layer thicknesses for first and second resonance modes.

\begin{tabular}{|l|c|c|c|c|c|c|}
\hline \multirow{2}{*}{$t_{C r}(\mathrm{~nm})$} & \multicolumn{3}{|c|}{ Mode 1 } & \multicolumn{3}{|c|}{ Mode 2 } \\
& $\Delta L_{1}(\mu \mathrm{m})$ & $\Delta(E I)_{1}$ & $\begin{array}{c}E I_{\text {corrected }} \\
\left(10^{-13}\right)\end{array}$ & $\Delta L_{2}(\mu \mathrm{m})$ & $\Delta(E I)_{2}$ & $E I_{\text {corrected }}$ \\
& 2.607 & 0.064 & 3.242 & 3.021 & 0.051 & 3.288 \\
97 & 2.031 & 0.057 & 3.526 & 2.083 & 0.071 & 3.473 \\
145 & 1.082 & -0.007 & 4.085 & 1.902 & -0.004 & 4.099 \\
\hline
\end{tabular}

\subsubsection{Pull-in voltage and ESS effect}

To increase the accuracy in predicting the pull-in voltages, in the static analysis (section 3.2.3.1), the dependence of the calculated pull-in voltages on the number of included modes is analysed. The analysis is done up to the fourth mode of vibration and it is found that the relative mean error is equal as for the case of inclusion of up to the third mode of vibration. A comparison between the relative mean errors for inclusion of various modes is shown in Table 3-VI. In the final calculation of the pull-in voltages of all the bimorph cantilevers, the first three modes are used. 
Table 3-VI. Dependence of pull-in voltage predictions on the number of modes included in the static analysis.

\begin{tabular}{|c|c|c|c|c|}
\hline Mode & $n=1$ & $n=2$ & $n=3$ & $n=4$ \\
Mean relative error \% & 6.961 & 6.439 & 6.431 & 6.431 \\
\hline
\end{tabular}

To calculate the pull-in voltages by the static analysis, the applied bias voltage is increased in small step sizes, and the numerical predictions are assessed. Figure 3.18 shows an example plot where tip deflection is plotted against applied $V_{\mathrm{DC}}$, showing the numerical runaway at $108.4 \mathrm{~V}$ for a bimorph of design length $100 \mu \mathrm{m}$ and a $t_{\mathrm{Cr}}$ of 145 $\mathrm{nm}$, clearly indicating it as the pull-in voltage. Similar analysis is being carried out for bimorphs of various lengths and Cr layer thicknesses and a discussion regarding it, is presented in section 3.7.1.

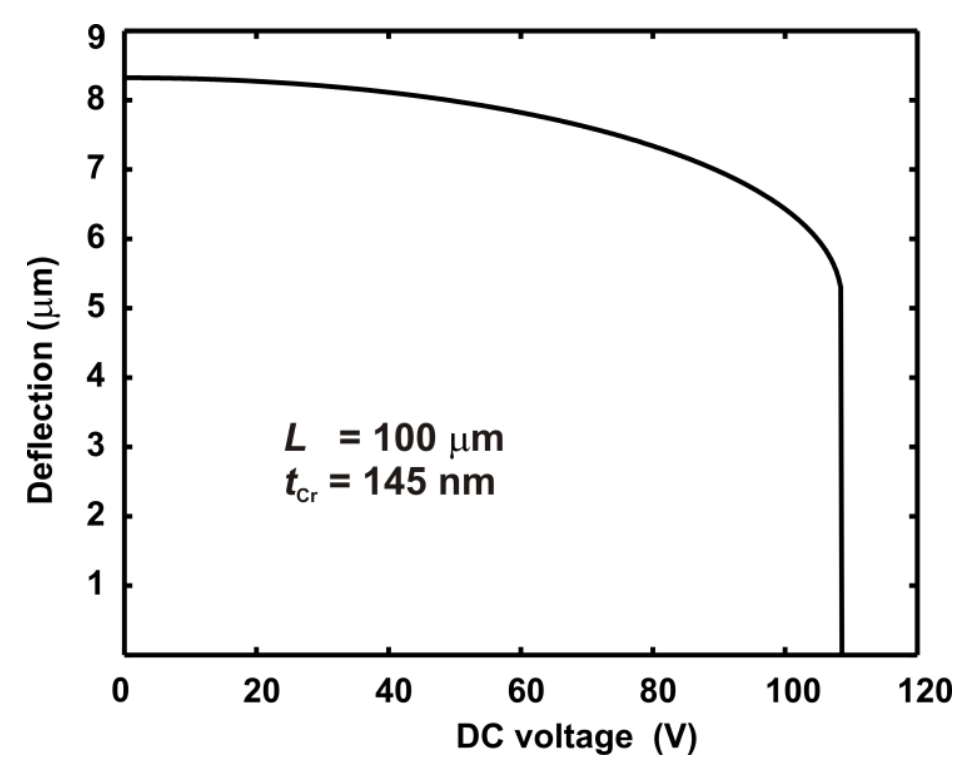

Figure 3.18. Static model analysis to calculate the pull-in voltage showing a pull-in voltage at $108.4 \mathrm{~V}$.

The measured resonance frequency shift by the ESS effect is compared with the dynamic model (section 3.2.3.2), for two different $t_{\mathrm{Cr}}(204 \mathrm{~nm}$ and $145 \mathrm{~nm}$ ) as shown in Figure 3.19 and in Figure 3.20. It can be seen that increasing the DC voltage decreases the resonance frequency and this variation is higher near the pull-in voltage. The solid line in the graphs represents the values calculated from the dynamic model and the starred points are from the measured data. The intersection of the curves with the horizontal axis indicates the pull-in voltage. As $V_{\mathrm{DC}}$ approaches $U_{\mathrm{pi}}$, the resonance frequency tends to zero since at this voltage the effective stiffness is zero. 
Chapter 3. Micro-bimorph cantilevers

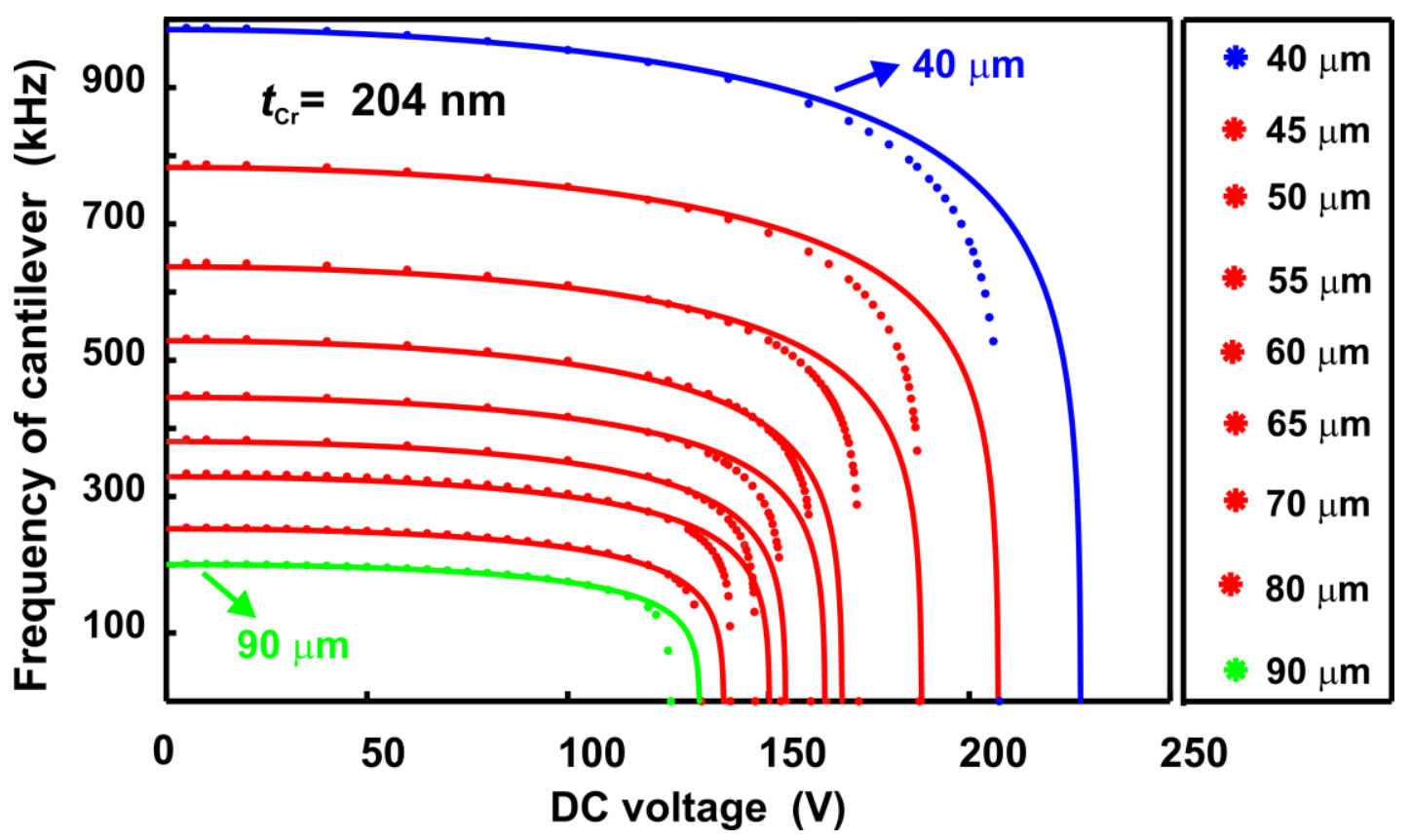

Figure 3.19. First mode resonance frequency shift due to ESS for the bimorphs of various (effective) lengths. (Cr layer thickness is $204 \mathrm{~nm}$ )

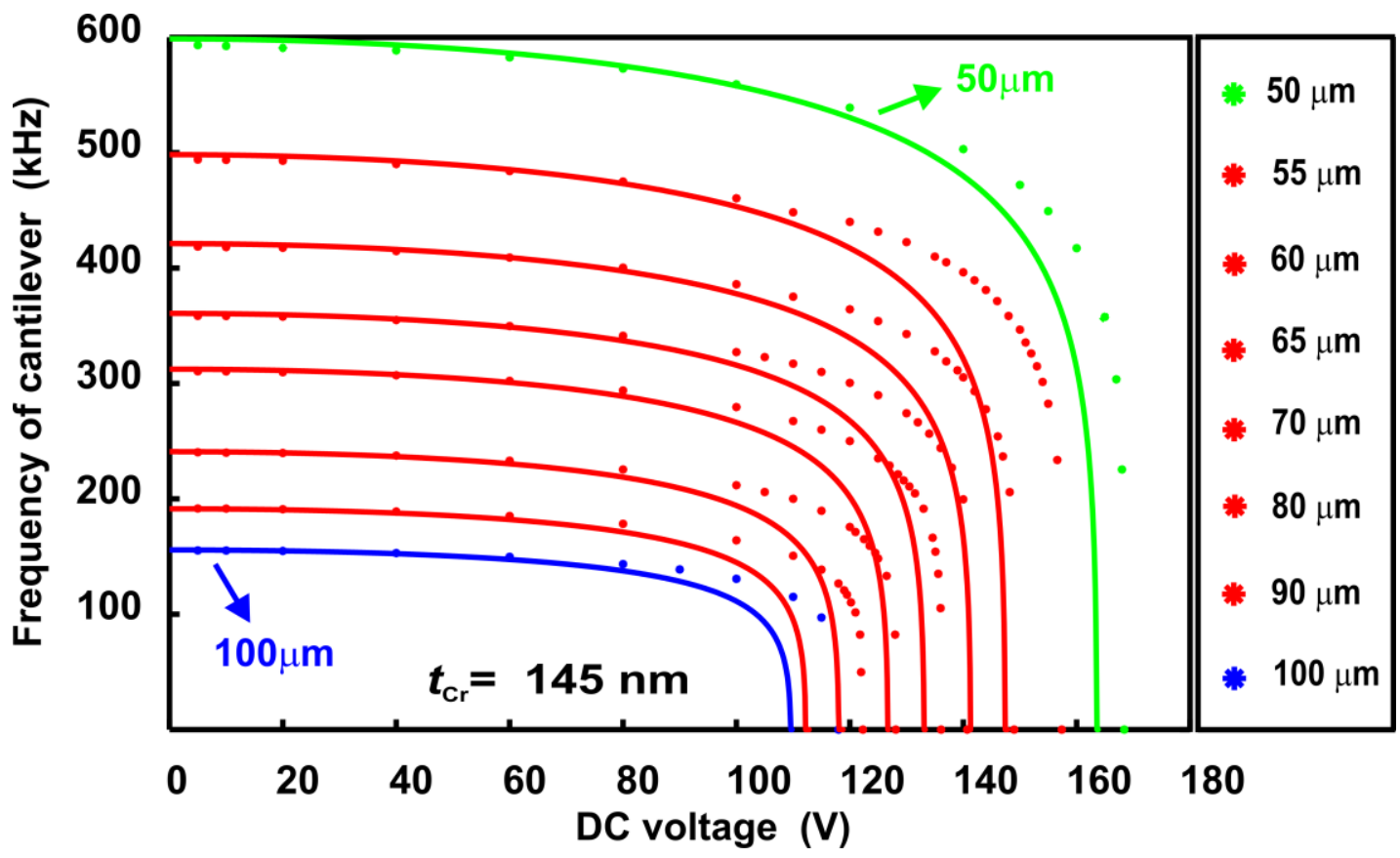

Figure 3.20. As Figure 3.19 for a Cr layer thickness of $145 \mathrm{~nm}$.

\subsection{Discussions}

\subsubsection{Pull-in voltage}

Pull-in voltages predicted by the static model are compared with the actual measured pull-in values. The model under predicted the pull-in voltage for $t_{\mathrm{Cr}} 97 \mathrm{~nm}$ and for $145 \mathrm{~nm}$, but over predicted for a $t_{\mathrm{Cr}}$ of $204 \mathrm{~nm}$ (Figure 3.21). 


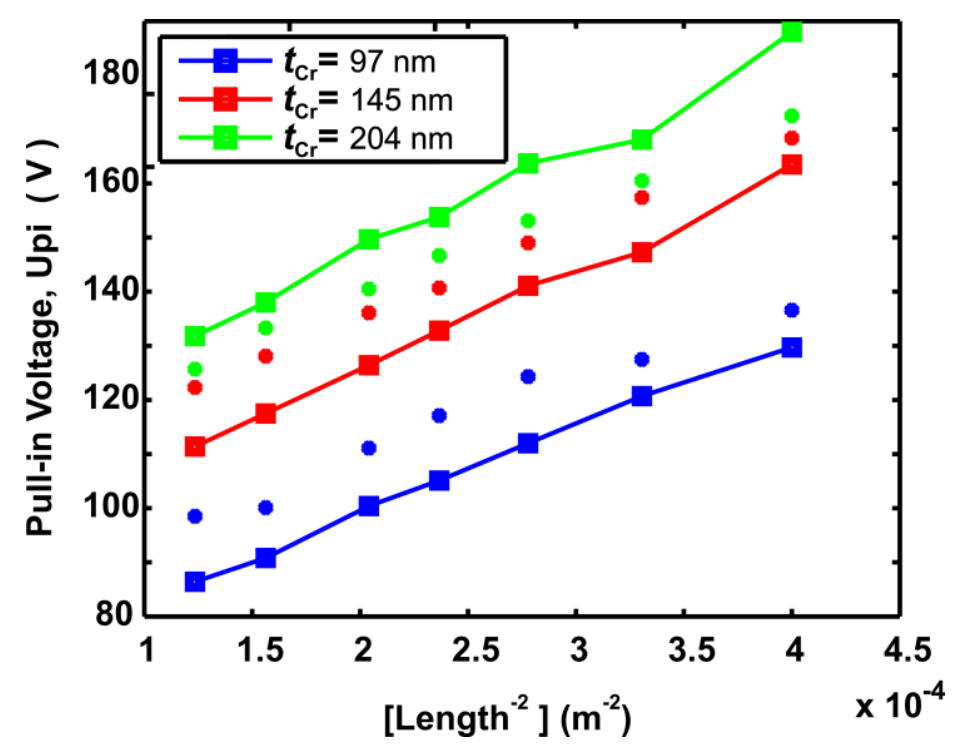

Figure 3.21. Comparison between measured pull-in voltages and those calculated from the static model for various Cr layer thicknesses. The starred values represent the measured data. The solid line is presented as a guide line to the eye for values predicted from the model.

The relative mean error between the measured values and the predicted values from the static analysis is calculated for different bimorphs as shown in Figure 3.22. The relative mean error lies around $6 \%$ for most of the beams. The error is smaller for shorter beams compared to longer beams.

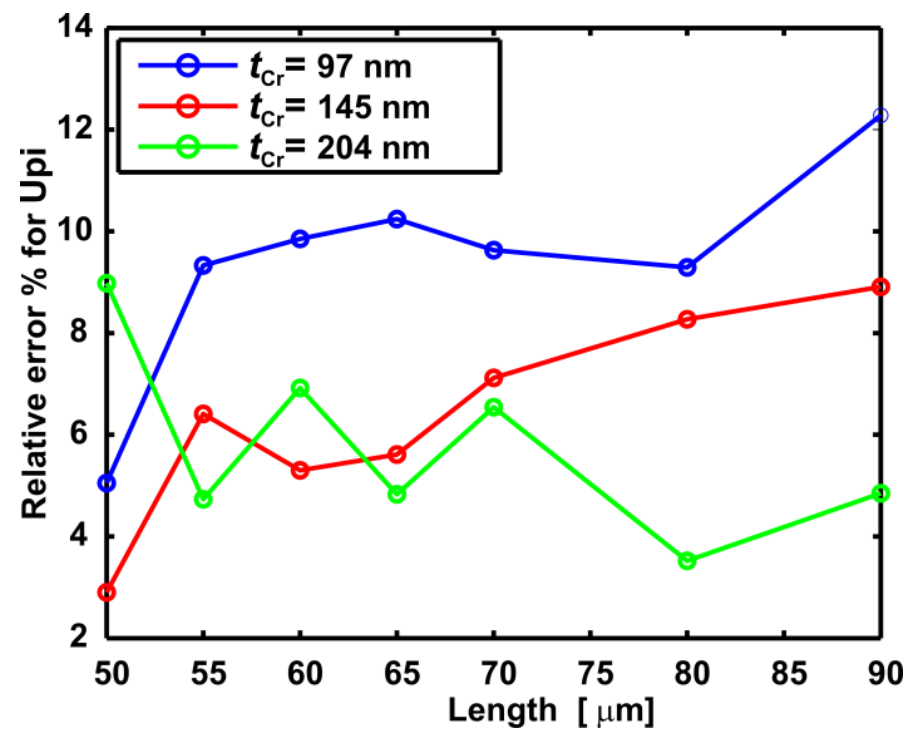

Figure 3.22. Relative errors in percentage for calculating the pull-in voltages from the static model and the measurements for various lengths and $\mathrm{Cr}$ layer thicknesses.

The dynamic model predicts the frequency shift accurately until $70 \%$ of its pull-in voltage. After $70 \%$, it either under predicted (for $t_{\mathrm{Cr}}=204 \mathrm{~nm}$ ) or over predicted (for $\left.t_{\mathrm{Cr}}=145 \mathrm{~nm}\right)$ the resonance frequencies. 
Chapter 3. Micro-bimorph cantilevers

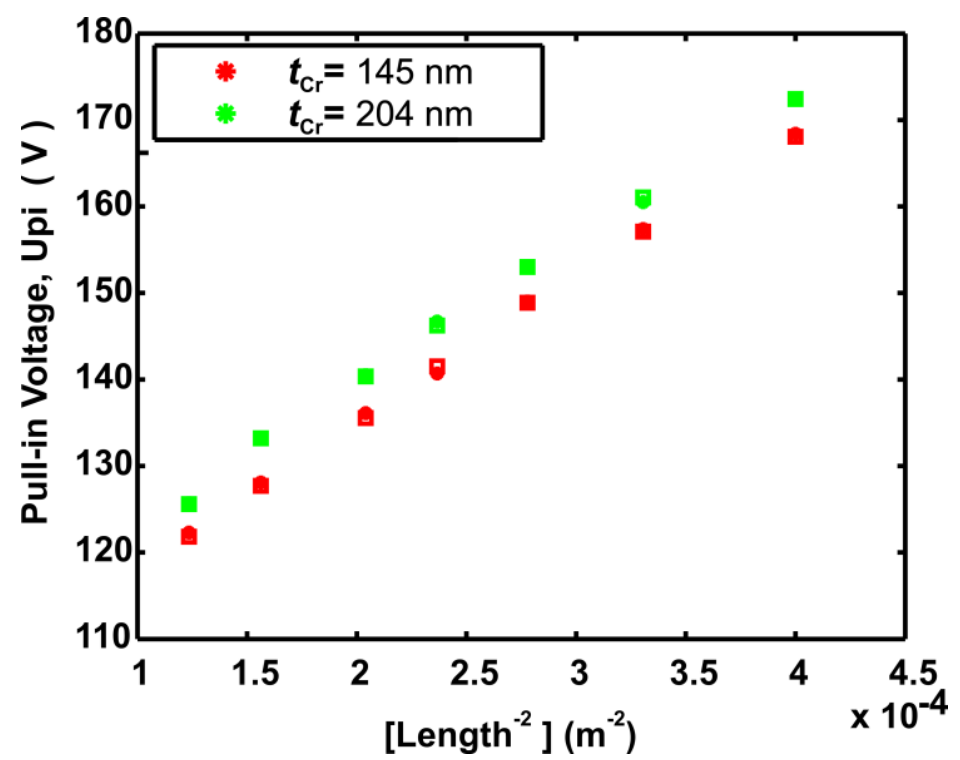

Figure 3.23. Comparison between measured pull-in voltages (starred values) and those determined from the ESS measurements (squared values) for two different Cr layer thicknesses.

It is seen that the values predicted from extrapolating the ESS measurements match well with the measured pull-in values (Figure 3.23). Moreover the normalised ESS measurements (Figure 3.13) show a very regular pattern, the lines virtually being on top of each other. Hence $f_{1}\left(V_{\mathrm{DC}}\right)$ obtained from the LDV measurements allows for an easy determination of $U_{\mathrm{pi}}$. This method becomes non-destructive when either a model or an appropriate curve fitting method can be used to predict the $V_{\mathrm{DC}}$ value for which $f_{1}\left(V_{\mathrm{DC}}\right)$ is zero. For example, looking at the normalised curves as shown in Figure 3.13 it is tempting to predict $U_{\mathrm{pi}} \approx \tilde{U} / \sqrt{0.9}$ where $\tilde{U}$ is the DC voltage for which the resonance frequency has dropped to $f_{1}(\tilde{U})=f_{1}(0) / \sqrt{2}$.

\subsubsection{Off-state deflection}

The off-state deflection of chromium coated cantilevers is much higher than it is required for the cantilevers which have to be used for mechano-optical modulation. An upward bending above $500 \mathrm{~nm}$ is undesirable as it moves the beam too far away from the optical evanescent field to be perturbed resulting in higher voltage requirements. One way to reduce the stress in the electrode layer and thereby the off-state deflection of the cantilever is to change electrode material to one having a low Young's Modulus. For this reason, an experimental analysis by changing the upper electrode from chromium $\left(E_{\mathrm{Cr}}=\right.$ $140 \mathrm{GPa}$ ) to gold $\left(E_{\mathrm{Au}}=78 \mathrm{GPa}\right)$ is carried out. However the gold still requires a thin chromium adhesive layer, otherwise it gets peeled-off from the $\mathrm{SiN}_{x}$ cantilever layer. Hence, the thickness of the adhesive layer is selected as small as possible. A combination of $\mathrm{Cr}(8 \mathrm{~nm})$ and $\mathrm{Au}(100 \mathrm{~nm})$ layers yielded an acceptable bending of the cantilever. A comparison between $\mathrm{Cr}$ and $\mathrm{Au}$ coated cantilevers is presented in Figure 3.24, for a 
cantilever length of $100 \mu \mathrm{m}$ and upper electrode thickness of $100 \mathrm{~nm}$. Further the study is extended to cantilevers of various lengths and it is concluded that gold coated cantilevers yielded much less off-state deflection than chromium coated cantilevers of similar length and similar electrode thickness (Figure 3.25). Hence the study in the following chapters is focussed on gold as the upper electrode material.

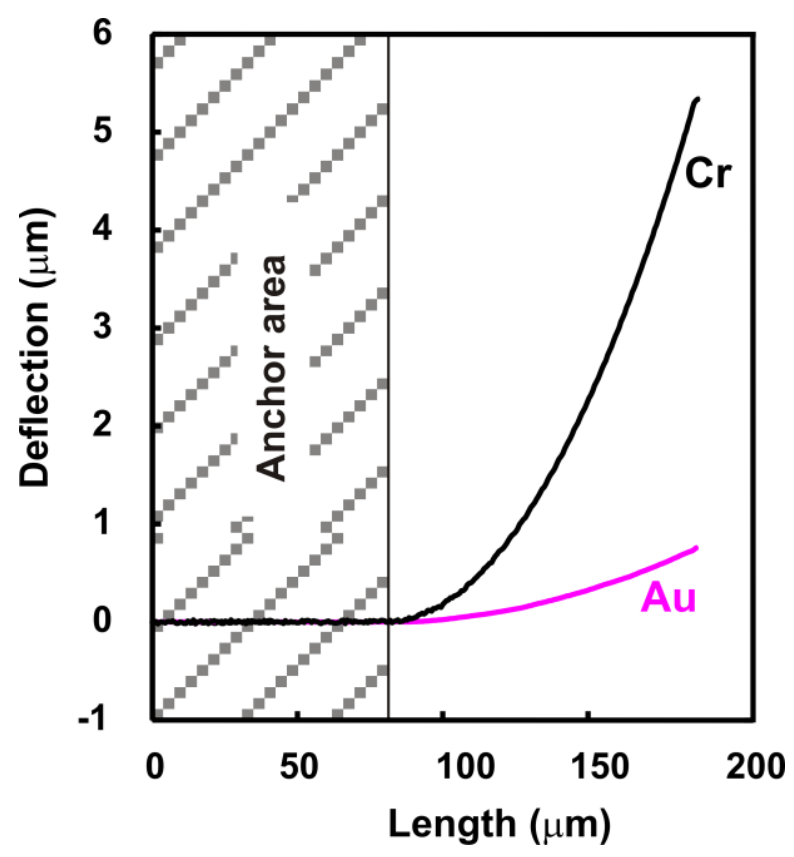

Figure 3.24. Comparison between off-state deflection of chromium and gold coated cantilevers having a design length of $100 \mu \mathrm{m}$ and $t_{\mathrm{Cr}}=100 \mathrm{~nm}$.

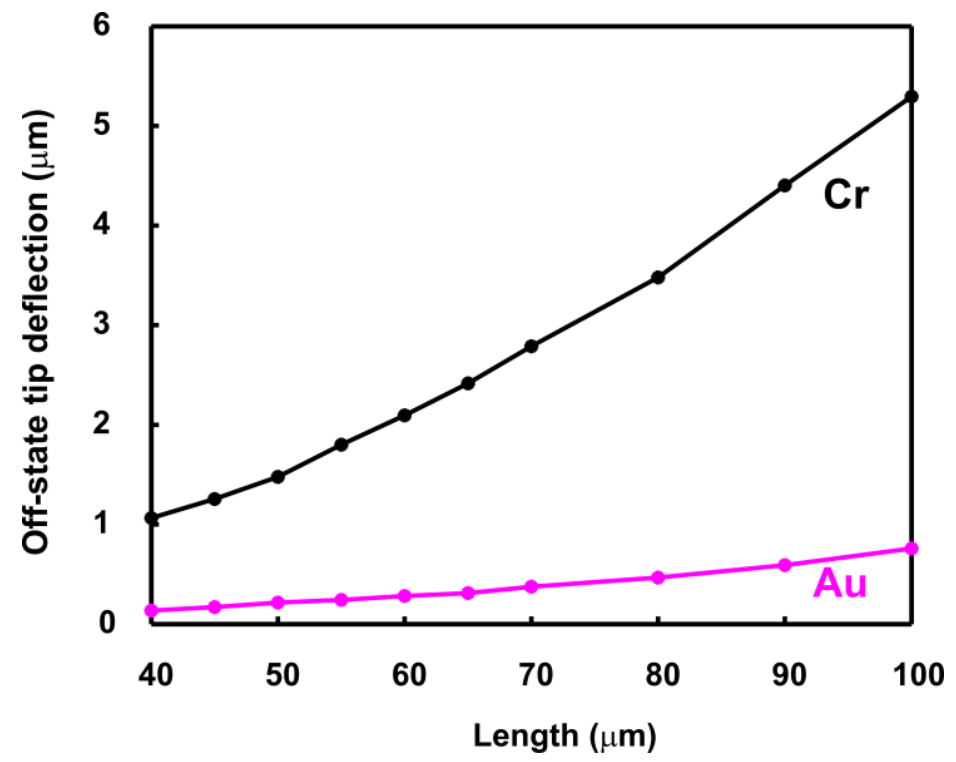

Figure 3.25. Comparison between off-state deflection of chromium and gold coated cantilevers for various lengths and $t_{\mathrm{Cr}}=100 \mathrm{~nm}$. 
Chapter 3. Micro-bimorph cantilevers

\subsection{Conclusions}

A detailed analysis on the resonance frequency as a function of the length and the upper electrode thickness of micro-curled bimorph cantilevers is presented. A Galerkin based step by step linearization method is developed to predict the static pull-in instability point. Further a dynamic model is developed to predict the shift in resonance frequencies due to an increase in DC bias voltage. The models are validated against data measured from bimorphs fabricated by surface micromachining techniques. The effect of undercut due to sacrificial layer etching is taken into account in the models. The measured shift in resonance frequencies by the electrostatic spring softening effect are used to extrapolate the resonance frequencies to zero which accurately predicts the pull-in instability point. Finally effect of upper electrode material on the off-state deflection of the cantilever is experimentally analysed and it is found that gold coated cantilevers yielded an acceptable off-state deflection than chromium coated cantilevers of same thickness.

\subsection{References}

[1] S. Schweizer, et al., "Thermally actuated optical microscanner with large angle and low consumption " Sensors and Actuators A: Physical, vol. 76, pp. 470-477, 1999.

[2] Y. Zhang, et al., "Thermally actuated microprobes for a new wafer probe card," IEEE Journal of microelectromechanical systems, vol. 8, pp. 43-49, 1999.

[3] T. Yasuda, et al., "Electrostatically driven micro elastic joints," in Proceedings of the IEEE/RSJ International Conference on Intelligent Robots and Systems, Pittsburgh, PA, 1995, pp. 242-245.

[4] G. Greitmann and R. A. Buser, "Tactile microgripper for automated handling of microparts " Sensors and Actuators A: Physical, vol. 53, pp. 410-415, 1996.

[5] T. Akiyama, et al., "Integrated atomic force microscopy array probe with metal-oxidesemiconductor field effect transistor stress sensor, thermal bimorph actuator, and on-chip complementary metal-oxide-semiconductor electronics," J. Vac. Sci. Technol. B vol. 18, pp. 2669-2675, 2000.

[6] V. Logeeswaran, et al., "Self-assembled microfabrication technology for 3D isotropic negative index material," in Proc.of SPIE 6393, 2006, pp. 639305-639310.

[7] J. H. Babaei, et al., "Integrable active microvalve with surface micromachined curled-up actuator," in Proc. 9th Int. Conf. Solid-State Sensors Actuators Chicago, 1997 pp. 833-836.

[8] D. C. Miller, et al., "Microrelay packaging technology using flip-chip assembly," in Proceedings of IEEE MEMS, 2000, pp. 265-270.

[9] C. Y. Lee and G. B. Lee, "Micromachine-based humidity sensors with integrated temperature sensors for signal drift compensation," J. Micromech. Microeng, vol. 13, pp. 620-627, 2003.

[10] W. Fang, et al., "Determining thermal expansion coefficients of thin films using micromachined cantilevers," Sensors and Actuators vol. 77, pp. 21-27, 1999.

[11] Y. H. Min and Y. K. Kim, "In situ measurement of residual stress in micromachined thin films using a specimen with composite-layered cantilevers," J. Micromech. Microeng, vol. 10, pp. 314-321, 2000.

[12] S. D. Senturia, "Chapter6:Energy-Conserving Transducers," in Microsystem Design, ed Massachusetts, United States: Kluwer Academic Publishers, 2003.

[13] R. T. Chen, et al., "A low voltage micromachined optical switch by stress-induced bending," in Proc.IEEE MEMS, 1999, pp. 424-428.

[14] C. Chang and P. Chang, "Innovative micromachined microwave switch with very low insertion loss," Sensors and Actuators A, pp. 71-75, 2000. 
[15] L. J. Kauppinen, "Compact integrated optical devices for optical sensor and switching applications," PhD Thesis, University of Twente, 2010.

[16] S. M. ChakkalakkalAbdulla, et al., "Mechanical tuning of optical race-track ring resonators," in Proc.Eurosensors XXIV, Linz, Austria, 2010.

[17] S. Iwamoto, et al., "Observation of micromechanically controlled tuning of photonic crystal line-defect waveguide," Applied Physics Letters, vol. 88, p. 011104, 2006.

[18] J. Cheng, et al., "Analytical and finite element model pull-in study of rigid and deformable electrostatic microactuators " J. Micromech. Microeng, vol. 14, pp. 57-68, 2004.

[19] S. C. Saha, et al., "Modelling of spring constant and pull-down voltage of non-uniform RF MEMS cantilever incorporating stress gradient," Sensors \& Transducers, vol. 98, pp. 54-68, 2008.

[20] R. K. Gupta, "Electrostatic pull-in test structure design for in-situ mechanical property measurements of microelectromechanical systems ", PhD Thesis, Massachusetts Institute of Technology, 1997.

[21] L. C. Wei, et al., "Analytical modeling for determination of pull-in voltage for an electrostatic actuated MEMS cantilever beam," in Proc. of the IEEE Int. Conf. on Semiconductor Electronics, Penang, Malaysia, 2002, pp. 233-238.

[22] Y. C. Hu and C. S. Wei, "An analytical model considering the fringing fields for calculating the pull-in voltage of micro curled cantilever beams," J. Micromech. Microeng, vol. 17, pp. 61-67, 2007.

[23] W. C. Chuang, et al., "Electromechanical behavior of the curled cantilever beam," J. Micro/Nanolith. MEMS MOEMS, vol. 8, pp. 0330201-8, 2009.

[24] A. Som 'and A. Ballestra, "Residual stress measurement method in MEMS microbeams using frequency shift data," J. Micromech. Microeng, vol. 19, pp. 0950231-16, 2009.

[25] H. A. C. Tilmans and R. Legtenberg, "Electrostatically driven vacuum-encapsulated polysilicon resonators Part II. Theory and performance," Sensors and Actuators A, vol. 45, pp. 67-84, 1994.

[26] MEMSnet. Available: http://www.memsnet.org/material/

[27] J. M. Gere, Mechanics of materials. Toronto, Canada: Thomson, 2006.

[28] M. Elwenspoek and R. Wiegerink, Mechanical Microsensors Springer Verlag, Germany, 2001.

[29] G. Genta, "Chapter12:Vibration of beams," in Vibration dynamics and control, ed New York: Springer, 2009.

[30] S.Timoshenko, Theory of plates and shells, Second ed.: McRraw Hill Book company, 1987.

[31] E. Volterra and E. C. Zachmanoglou, Dynamics of Vibrations Ohio, Charles E. Merrill Books, Inc.,Columbus, 1965.

[32] E. M. Abdel-Rahman, et al., "Characterization of the mechanical behavior of an electrically actuated microbeam " J. Micromech. Microeng, vol. 12, pp. 759-766, 2002.

[33] G. Rezazadeh, et al., "Application of piezoelectric layers in electrostatic MEM actuators: controlling of pull-in voltage," Microsystem Technologies, vol. 12, pp. 1163-1170, 2006.

[34] N. A. Bobylev, et al., "Chapter3:Convergence of numerical procedures," in Approximation procedures in nonlinear oscillation theory, ed Berlin: Walter de Gruyter\&Co, 1994.

[35] N. Tas, et al., "Stiction in surface micromachining," Journal of Micromechanics and Microengineering, vol. 6, pp. 385-397, 1996.

[36] J. G. E. Gardeniers, et al., "LPCVD silicon-rich silicon nitride films for applications in micromechanics, studied with statistical experimental design," Journal of V acuum Science and Technology A: Vacuum, Surfaces and Films, vol. 14, pp. 2879-2892, 1996.

[37] R. Legtenberg and H. A. C. Tilmans, "Electrostatically driven vacuum-encapsulated polysilicon resonators Part I. Design and fabrication," Sensors and Actuators A, vol. 45, pp. 57-66, 1994.

[38] G. G. Stoney, "The tension of metallic films deposited by electrolysis," Proc. R. Soc. Lond. $A$, vol. 82, pp. 172-175, 1909.

[39] W. C. Chuang, et al., "Review on the modeling of electrostatic MEMS," Sensors, vol. 10, pp. 6149-6171, 2010. 
Chapter 3. Micro-bimorph cantilevers 


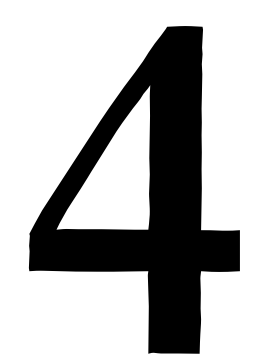

\section{Micro-cantilever integrated micro-ring resonator: Fabrication}

This chapter presents fabrication details of integrating a microcantilever with a micro-ring resonator. A detailed discussion on various fabrication-related issues is carried out: on which a major one is related with the formation of 'stringers' which are etch residues at surface steps. Two novel stringer elimination methods are presented and compared with various existing methods known in semiconductor manufacturing.

Part of this chapter is submitted for publication as S.M.C. Abdulla, H.V. Jansen, E. Berenschot, N.R. Tas, M. Dijkstra, M.J. de Boer, Y. Zhao and G.J.M. Krijnen, "Stringer elimination methods in surface nano and micromachining". 
Chapter 4. Micro-cantilever integrated micro-ring resonator: Fabrication

\subsection{Introduction}

Microring resonators are highly promising building blocks in the field of integrated optical systems, having a wide range of applications including lasers [1], optical switches [2], tunable wavelength filters [3], add/drop multiplexers [4] and biosensors [5]. They have been attracting attention in recent years due to their compactness, high quality factor, ease of fabrication, and ability for dense integration. Tuning of a microring resonator makes it possible to use it as an active element, which can have ample applications in future optical communication networks. Furthermore, tuning allows for compensation of small fabrication-induced variations of geometrical parameters, changes in material properties by temperature variations and so on. One of the tuning methods is to change the refractive index of the materials of the ring resonator and thereby the ring's resonance wavelength. The resonator can be tuned by a variety of means e.g thermooptic, electro-optic, plasma dispersion, or electromechano-optic [6-7]. Among these methods, opto-mechanical tuning by electrostatic actuation has the characteristics of potentially providing a broad wavelength tuning range and requiring very little energy for operation.

In general both the real and imaginary parts of the effective index of a guided mode in a dielectric waveguide will be affected by the presence of an object -such as a cantilever- in its evanescent field. If the waveguide is part of a ring resonator, a change of the real part of the effective index causes a shift of the optical resonance wavelength, whereas a change of the imaginary part translates into a change of the optical quality factor. Micromechanically-actuated wavelength-selective on/off-switching of a siliconnitride micro-ring resonator has been demonstrated before, by moving an aluminum membrane into and out of the evanescent field of a ring waveguide, using a $2 \mathrm{kHz}, 0-30 \mathrm{~V}$ square-wave driving signal [2]. Since the lossy aluminum affects mainly the imaginary part of the effective index, this device can only be used as an on/off switch but cannot be used for wavelength tuning. In a different approach, $27 \mathrm{~nm}$ tuning at $1565 \mathrm{~nm}$ (or 1.7\% wavelength tuning) of a silicon-nitride ring resonator has been demonstrated by perturbing the evanescent field with an external silica fiber probe [3]. Obviously, the drawback of this approach is the lack of monolithic integration.

The aim of the current study is to fabricate, characterize, and demonstrate the feasibility of tuning or switching of optical signals using a silicon racetrack ring resonator integrated with a movable dielectric micro-cantilever. On application of a voltage between the cantilever and the substrate, the former is pulled into the evanescent field. This increases the effective index of the resonator waveguide, resulting in a shift of the resonance wavelength. This CMOS-compatible device is designed for operation in the 
optical communications C-band (1530-1565 nm), and the waveguides of the ring resonator are designed to be single mode for TE-polarized light at a wavelength of 1550 nm.

The organization of this chapter is as follows. The next section gives a brief description of the basic principle of the device. Section 4.3 discusses the fabrication technology for integrating dielectric cantilevers with optical racetrack ring resonators. Then, a discussion about one of the fabrication-related issues - the stringer formation and its elimination - is presented in section 4.4. Finally section 4.5 provides a summary and conclusions of the chapter. Throughout this chapter, off-state of the device refers to the situation in which there is no electrical signal, ie. the cantilever is in the upper position, which in turn is an optical on-state, in which the resonator is virtually unperturbed by the cantilever.

\subsection{Basic principle}

The modal field of an optical waveguide has an exponentially decaying evanescent field, which typically extends only a few hundred nanometers into the (air) cladding. The cantilever (length between 40 and $100 \mu \mathrm{m}$ and $10 \mu \mathrm{m}$ in width) is a bimorph consisting of an upper layer acting as the electrode and a lower layer acting as the dielectric. In the offstate, as shown in Figure 4.1C, the thermal- and deposition-induced stresses during fabrication make it to bend upward (see section 3.5.2 also); the stress is optimized such that the tip of the bimorph is hardly affecting the modal propagation properties, i.e., the waveguide is unperturbed. On application of a voltage between the top electrode and the substrate, the cantilever is pulled towards the resonator and the air is partially replaced by the dielectric material of higher refractive index. This changes the modal propagation and hence tunes the resonator. The needed deflection of the bimorph is designed to be less than $500 \mathrm{~nm}$ to reduce the voltage required to pull the cantilever into the evanescent field. Figure 4.1 shows a schematic illustration of the integrated device, with (A) top view and (B), (C) different cross-sectional views and Figure 4.2 shows a close-up of Figure 4.1C. 
Chapter 4. Micro-cantilever integrated micro-ring resonator: Fabrication
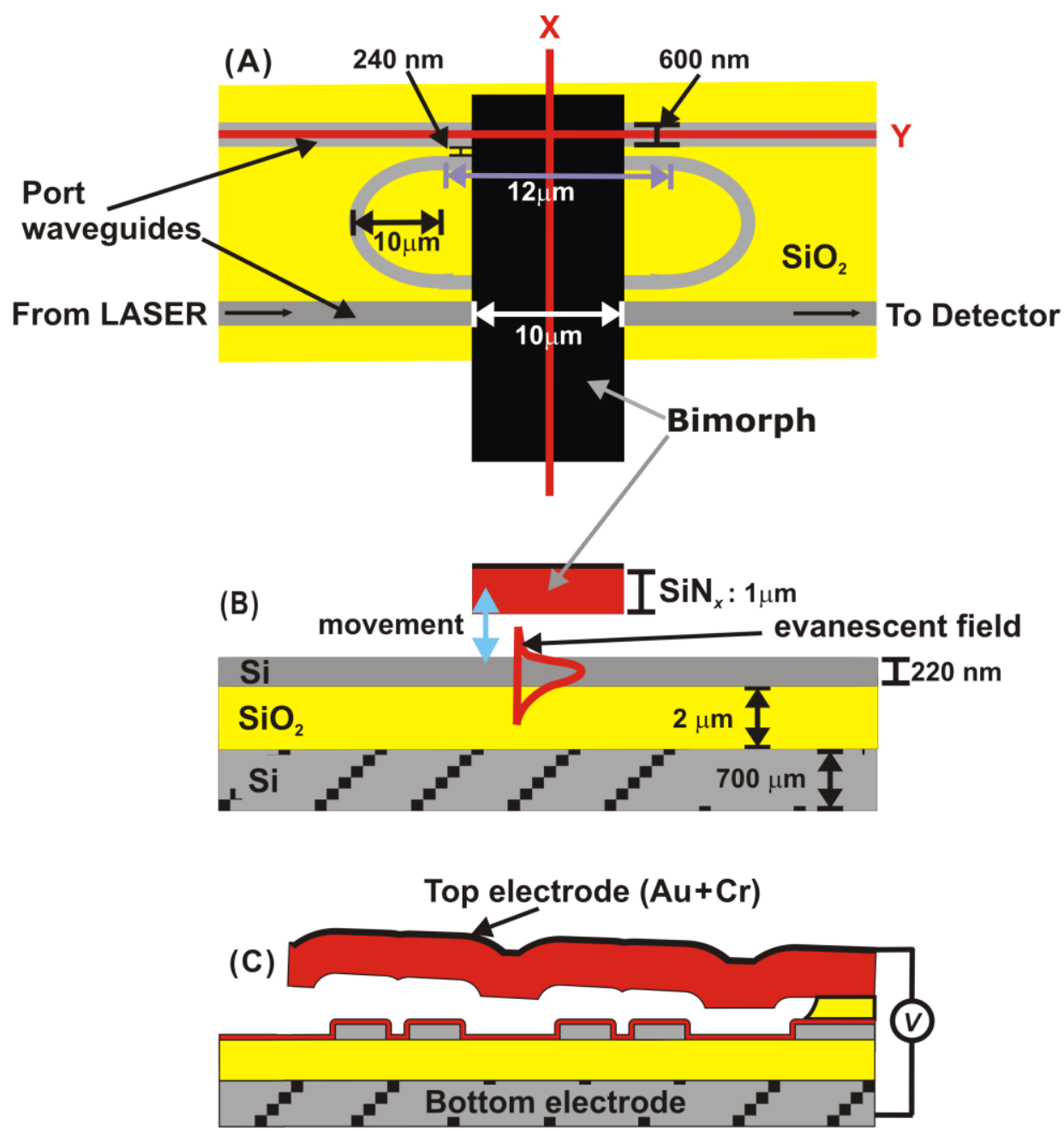

Figure 4.1. Schematic illustration of the integrated device. (A) top view; (B) cross-sectional view through $Y$; and (C) cross-sectional view through $X$.

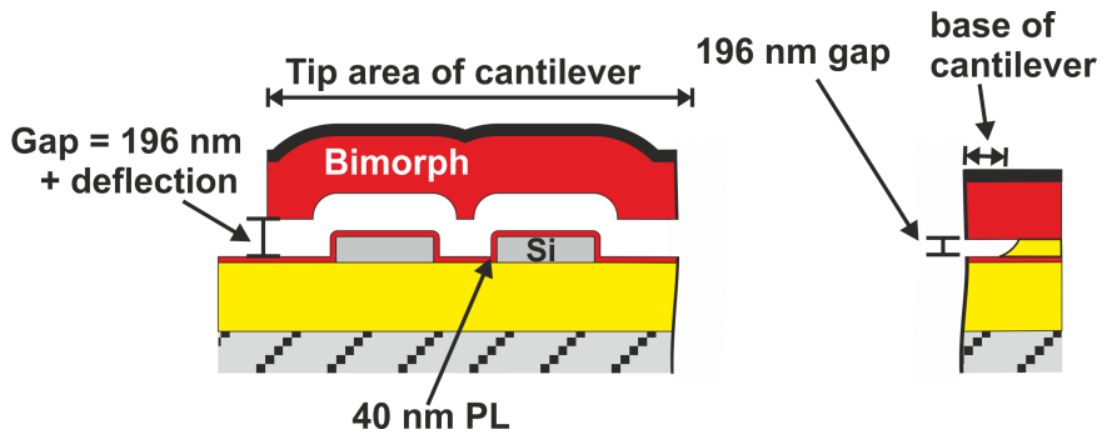

Figure 4.2. Close-up of Figure 4.1C.

\subsection{Fabrication}

\subsubsection{Optical device fabrication}

Racetrack ring resonators were designed with a bend radius of $10 \mu \mathrm{m}$ and a straight section length of $12 \mu \mathrm{m}$ [7]. As shown in Figure 4.1, the gap between the port (or 
access) waveguide and the straight section of the ring is $240 \mathrm{~nm}$. The length of the access waveguides is $6000 \mu \mathrm{m}$ with gratings at both end to couple light vertically [8]. The width of the ring and its access waveguides is $600 \mathrm{~nm}$. However, $2200 \mu \mathrm{m}$ away from the centre of the ring, in both directions, the width of the waveguides increases gradually and reaches $10 \mu \mathrm{m}$ at the location of the grating couplers. The resonators and waveguides have been fabricated on a $200 \mathrm{~mm}$ silicon-on-insulator (SOI) wafer using a $193 \mathrm{~nm}$ deepUV lithography process by the silicon photonics platform ePIXfab [9]. The thickness of the silicon device layer is $220 \mathrm{~nm}$ and that of the silicon handle wafer is $700 \mu \mathrm{m}$. The ring resonators were formed by patterning and etching the Si device layer and stopping on the $2 \mu \mathrm{m}$ thick buried oxide layer, which serves as the bottom cladding of the waveguides.

\subsubsection{Micro-cantilever integration}

Figure 4.3 shows the fabrication flow for wafer-scale integration of bimorph cantilevers with a racetrack ring resonators. It is a two-mask process using positive photoresist for the UV lithography. (a) Starting with a SOI wafer, in which the optical resonators have been previously fabricated [9], (b) $40 \mathrm{~nm}$ low-stress silicon-rich nitride $\left(\mathrm{SiN}_{x}\right)$ is deposited using low-pressure chemical vapour deposition (LPCVD) method [10]. It serves as a protective layer (PL) for the underlying oxide cladding layer and the waveguides. This $\mathrm{SiN}_{x}$ layer is removed from the backside of the wafer by reactive ion etching (RIE) to later on have access to the bottom electrode. Next, a $200 \mathrm{~nm}$ LPCVD tetraethyl orthosilicate (TEOS) oxide sacrificial layer (SL) is deposited conformally, at $700^{\circ} \mathrm{C}$. The thickness is chosen such that the formation of etch residues, known as stringers (see section 4.4) can be reduced or even prevented. Then $1.1 \mu \mathrm{m} \mathrm{LPCVD} \mathrm{SiN}_{x}$, which will later be structured into the micro-cantilever, is deposited, at $850^{\circ} \mathrm{C}$. On the front side of the wafer, $50 \mathrm{~nm}$ gold is sputtered as the top electrode using $8 \mathrm{~nm}$ chromium as adhesive. (c) Subsequently, the first resist mask (OiR 907-17, Fujifilm) is used to pattern the metal layers; Au is etched in gold etch solution at $30^{\circ} \mathrm{C}(\mathrm{KI}=132 \mathrm{~g}$, $\mathrm{I} 2=18 \mathrm{~g}$, DI $=1200 \mathrm{ml}$, in which glycerin $(600 \mathrm{ml})$ is added to reduce the excessive undercut), and $\mathrm{Cr}$ is wet etched in chromium etchant (MERCK 111547.2500). (d) The second resist mask (OiR 908-35, Fujifilm) is used for patterning the $\mathrm{SiN}_{x}$ into the cantilevers by RIE [11] (Elektrotech PF340 at $10^{\circ} \mathrm{C}, 75 \mathrm{~W}, 10$ mTorr, $25 \mathrm{sccm} \mathrm{CHF} 3$ and $5 \mathrm{sccm} \mathrm{O}$ ). The resist is first plasma etched for 1 minute (TEPLA 300E, $200 \mathrm{sccm} \mathrm{O}$, $500 \mathrm{~W}, 1.2 \mathrm{mbar}$ ) to remove the fluorocarbon contamination produced by RIE [12] which, if remaining, might hamper the subsequent 20 minutes $\mathrm{HNO}_{3}(99 \%)$ resist stripping. (e) Finally, sacrificial layer etching (SLE), using buffered hydrofluric acid (BHF, $\mathrm{NH}_{4} \mathrm{~F}: \mathrm{HF}=7: 1$ ) followed by freeze drying [13] (see also Appendix I), is applied to release the cantilevers. The SLE is divided into three steps in which the second one consists of 
an ultrasonic cleaning step, as described in section 4.4.2.4, to remove the $\mathrm{SiN}_{x}$ stringers. A detailed fabrication flow of this process is presented in Appendix II-Table II. An SEM image of a successfully integrated device is shown in Figure 4.4.

(a)

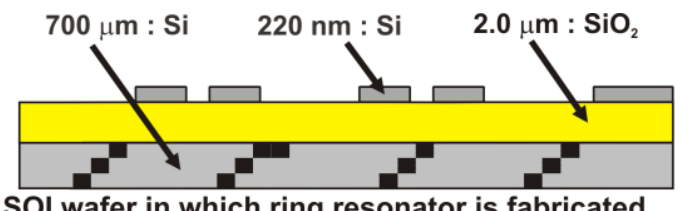

SOI wafer in which ring resonator is fabricated

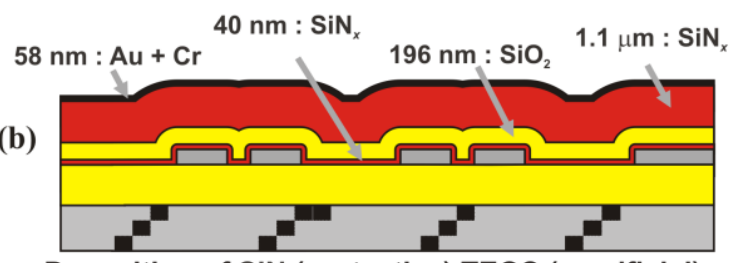

Deposition of $\mathrm{SiN}_{x}$ (protective), TEOS (sacrificial), $\mathrm{SiN}_{x}$ (cantilever) and metal layers

(c)

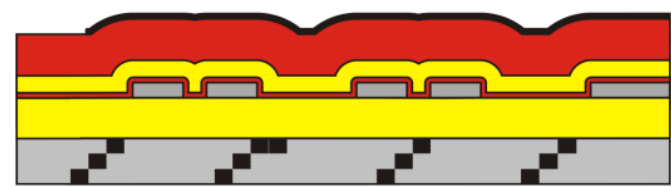

Patterning and wet etching of metal layers (mask 1)

(d)

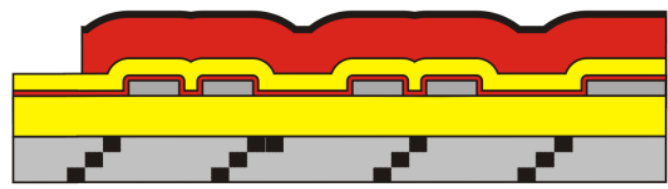

Patterning and dry etching of $\mathrm{SiN}_{x}$ layer (mask 2)

(e)

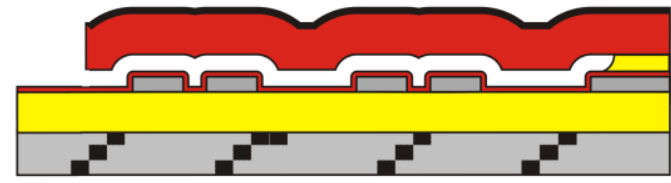

Sacrificial layer etching to release bimorphs

Figure 4.3. Fabrication flow of a silicon racetrack resonator integrated with a tuning cantilever. 


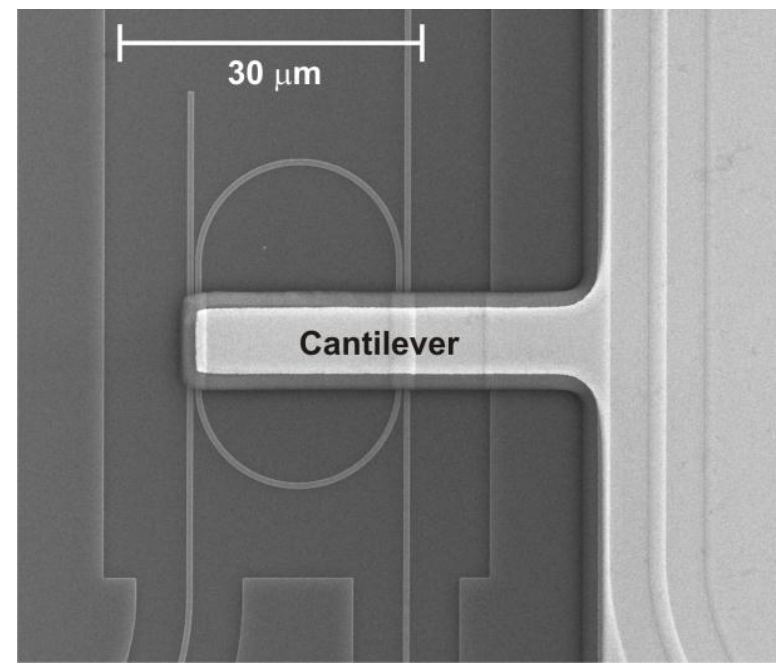

Figure 4.4. SEM image of a $40 \mu \mathrm{m}$ long cantilever successfully integrated with the racetrack ring resonator.

\subsubsection{Materials selection}

Conformal layers with high wafer uniformity are essential for successful device fabrication with high yield. Therefore, the composition and thickness of each layer was chosen carefully. Table 4-I gives the measured variation in thickness across the wafer and the refractive index for various conformal layers used for the fabrication. By virtue of its high uniformity, LPCVD TEOS oxide was chosen as the SL. Furthermore, it is important to protect the thermal oxide lower cladding layer (i.e. the $2 \mu \mathrm{m}$ buried oxide) during SLE. If unprotected, it will be attacked by BHF which will result in under-cutting the waveguides, thereby destroying the devices (see section 4.4.2.1). The PL was selected to be $\mathrm{SiN}_{x}$ because of its high resistance (etch rate $0.5 \mathrm{~nm} / \mathrm{min}$ ) to the $\mathrm{BHF}$ etchant used for SLE of TEOS oxide $(180 \mathrm{~nm} / \mathrm{min})$. During the subsequent $\mathrm{SiN}_{x}$ deposition at $850^{\circ} \mathrm{C}$, the TEOS oxide SL is annealed which reduces the etch rate to an estimated value of 150 $\mathrm{nm} / \mathrm{min}$. The required SL etch time of a cantilever of $10 \mu \mathrm{m}$ wide $(5000 / 150 \sim 33 \mathrm{~min})$ defines the minimum thickness of the PL to be approximately $17 \mathrm{~nm}(33 \times 0.5)$. However, an overetching of 19 minutes is performed to ensure that the $\mathrm{SiO}_{2}$ beneath the cantilever is completely removed. Including this extra time and a safety time to counteract delays caused by layer non-uniformity, a PL thickness of $40 \mathrm{~nm}$ is selected. Gold (Au) is chosen as the top electrode by virtue of its relatively low residual stress, which in turn reduces the upward bending of the cantilever. But it needs an additional layer to promote sufficient adhesion with the nitride underneath. The SLE step in BHF excludes to select metals like titanium, tantalum, aluminum etc as either the top electrode or as the adhesive layer. A reasonable candidate is chromium $(\mathrm{Cr})$, although this material is known to have large built-in stress. Therefore, it has to be as thin as possible. It is found that a combined thickness of $58 \mathrm{~nm}(50 \mathrm{~nm} \mathrm{Au}$ and $8 \mathrm{~nm} \mathrm{Cr}$ ) gives an acceptable off-state deflection. 
Chapter 4. Micro-cantilever integrated micro-ring resonator: Fabrication

Table 4-I. Measured thickness variation across the wafer and the refractive index of different conformal layers.

\begin{tabular}{|c|c|c|c|}
\hline Layer (function) & Thickness (nm) & $\begin{array}{c}\text { Standard 3 } \sigma \text { deviation } \\
\mathbf{( n m})\end{array}$ & $\begin{array}{c}\text { Measured refractive } \\
\text { index (n) at } \mathbf{6 3 3} \mathbf{~ n m}\end{array}$ \\
$\operatorname{SiN}_{x}$ (Protective) & 40 & 2 & 2.20 \\
$\operatorname{TEOS}_{\text {(Sacrificial) }}$ & 196 & 2 & 1.45 \\
$\operatorname{SiN}_{x}($ Cantilever) & 1097 & 50 & 2.25 \\
\hline
\end{tabular}

\subsubsection{Micro-cantilever geometry}

The micro-cantilevers are designed to be rectangular in cross-section having a fixed width of $10 \mu \mathrm{m}$. However due to the retrieval of photoresist during RIE [14], the sides of the cantilevers are not perfectly rectangular, but in trapezoidal shape. Figure 4.5 shows the different views of the cantilever, integrated (A) with a micro-ring resonator and (B) with straight waveguides, showing the tapering of the sides. Due to this, the width of the cantilever is varying from its top to bottom, with the measured variation in one side being $\sim 1.3 \mu \mathrm{m}$.

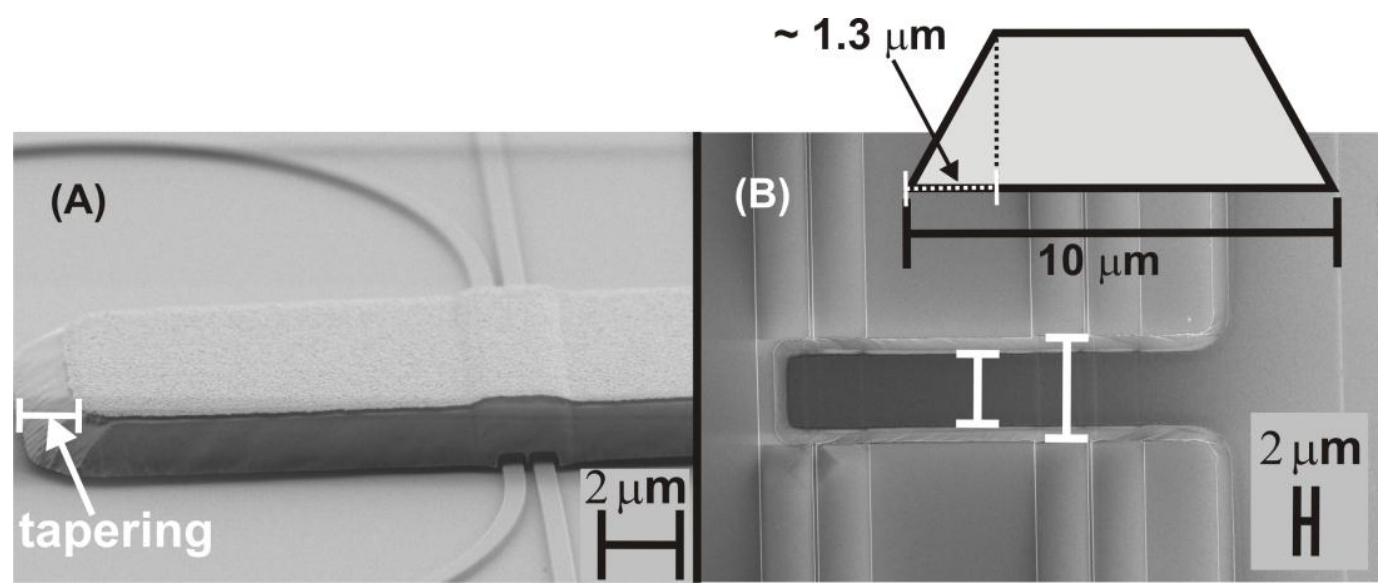

Figure 4.5. SEM images showing the tapering on the sides of the cantilever due to photoresist retrieval during RIE.

Apart from this, the actual length of the fabricated cantilever is increased from the designed length of the mask due to the undercut at the bond pads caused by the SLE (see section 3.6.1). The undercut, which was measured with an optical microscope to be 5.5 $\mu \mathrm{m}$, will result in an increase of the effective cantilever length with associated lower resonance frequency and pull-in voltage. 


\subsubsection{Protrusions on the cantilever}

Conformal layer deposition results in the formation of protrusions ("ridges") on the cantilever that penetrate into the gap between the ring resonator and the port waveguides. Figure 4.6 shows a ridge protruding into the gap between the ring and the waveguide in the cantilever off-state. The sharpness of the ridges will reduce with increasing SL thickness. Since the coupling between the ring and the straight waveguide is strongly affected by the variation in distance between them [15], the effect of these ridges on the coupling strength is a topic which needs further investigation.

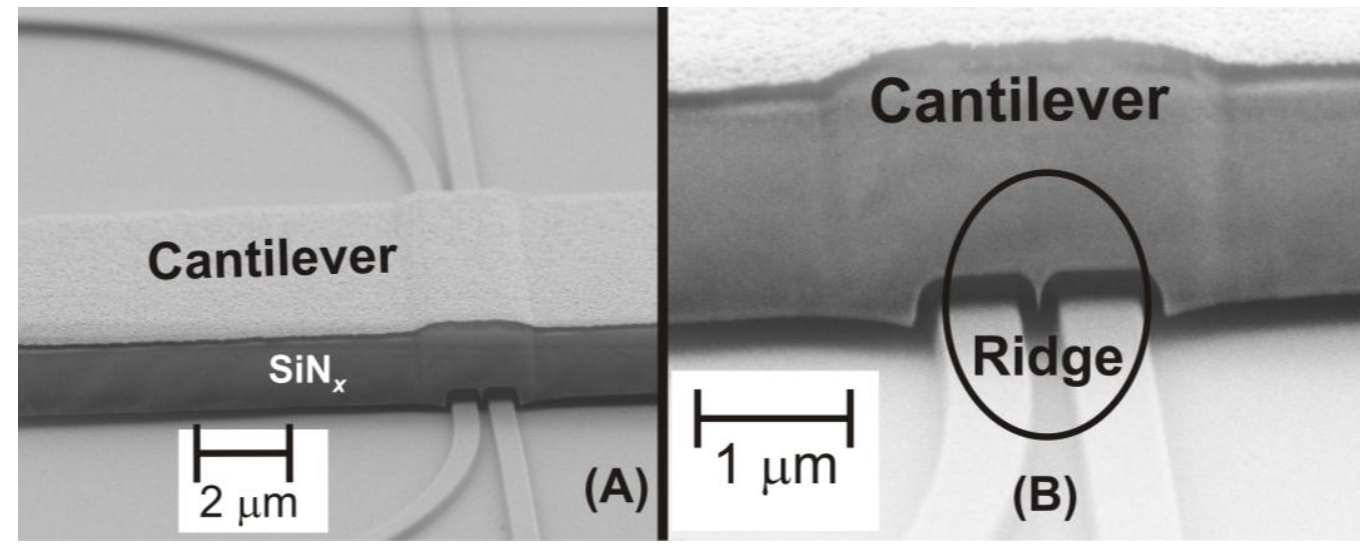

Figure 4.6. (A) SEM image showing the released cantilever in the off-state with its pin (B) protruding into the gap between a port waveguide and a straight resonator waveguide section.

\subsection{Fabrication related issues: Stringer formation and removal}

Conformal deposition on a patterned surface is widely used in micro and nanomachining. As the size of the steps caused by the pattern shrink into the nano scale, some of the usually minor problems related with etching may become major. One such a problem is the formation of stringers, which are unwanted by-products running parallel to a stepped surface [16-19]. If unresolved, stringers can seriously fail the performance of devices. In semiconductor or capacitive MEMS devices they can short circuit conducting structures; in optical devices they can create substantial losses, whereas in mechanical devices they can seriously obstruct movements. This makes it of utmost importance to develop efficient methods to eliminate stringers, especially on the nanoscale where they are more likely to cause failure. In this section, a detailed analysis is carried out on stringer formation for the process described in section 4.3 for fabricating the integrated optical microring resonator.

When a voltage is applied between the metallic layer and the substrate, the movable cantilever is forced into the evanescence field of a waveguide and will modulate the optical signals therein. So, the metallic layer has to be in close proximity of the substrate to allow for sufficient displacement at a relative small voltage. On the other 
hand, the dielectric $\mathrm{SiN}_{x}$ layer has to be thick enough to prevent optical loss caused by the interaction of the evanescent field with the metal electrode. Furthermore, to achieve a significant optical effect by a small displacement, the vertical gap between the cantilever and the micro-ring resonator has to be on the order of the evanescent field decay length. In the used fabrication process flow, this thin gap is defined by the thickness of TEOS oxide sacrificial layer which will be locally sacrificed in a final process step. These design rules lead to an etching step in the process, where a thick $\mathrm{SiN}_{x}$ layer has to be etched selectively with respect to a thin TEOS oxide SL. It is important that this SL survives the $\mathrm{SiN}_{x}$ etch, as it protects the underlying layers, such as the waveguides, too.

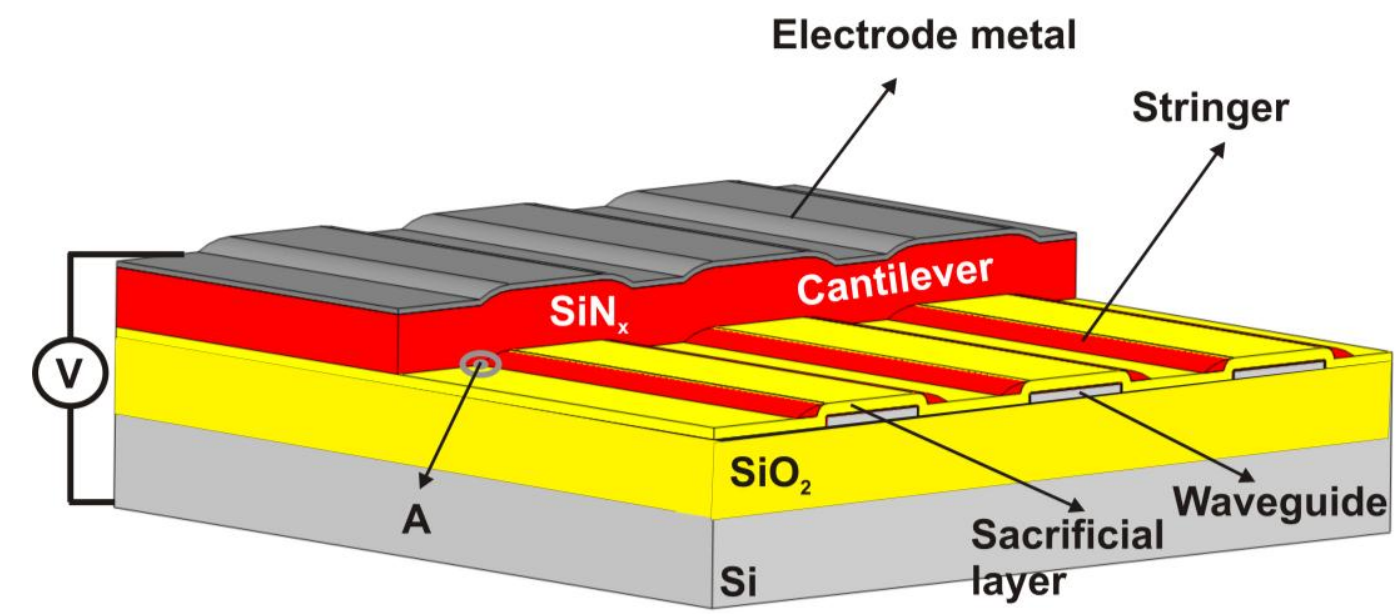

Figure 4.7. Stringers running parallel to waveguides and attached to the side of a $\operatorname{SiN}_{\mathbf{x}}$ cantilever (at position 'A') (Image source: E. Berenschot, TST Group, University of Twente, The Netherlands).

One of the problems related to insufficient selectivity in this etching procedure is the formation of unwanted stringers. They run parallel to the waveguides and are attached to the cantilever, as marked 'A' in Figure 4.7. Upon SLE they are released from the sides of the waveguide, but are still attached to the cantilever at position 'A'. This makes the stringers to be loosely bound to the cantilever close to the optical device to be modulated, posing serious problems to the mechanical, electrical and optical performance of the integrated device: Figure 4.8A shows SEM image after unsuccessful device release, displaying these undesirable stringers. Hence proper methods have to be implemented to eliminate stringers for a successful realisation of the final device: Figure $4.8 \mathrm{~B}$ shows a "clean" device obtained using the sonification technique described in section 4.4.2.4. 


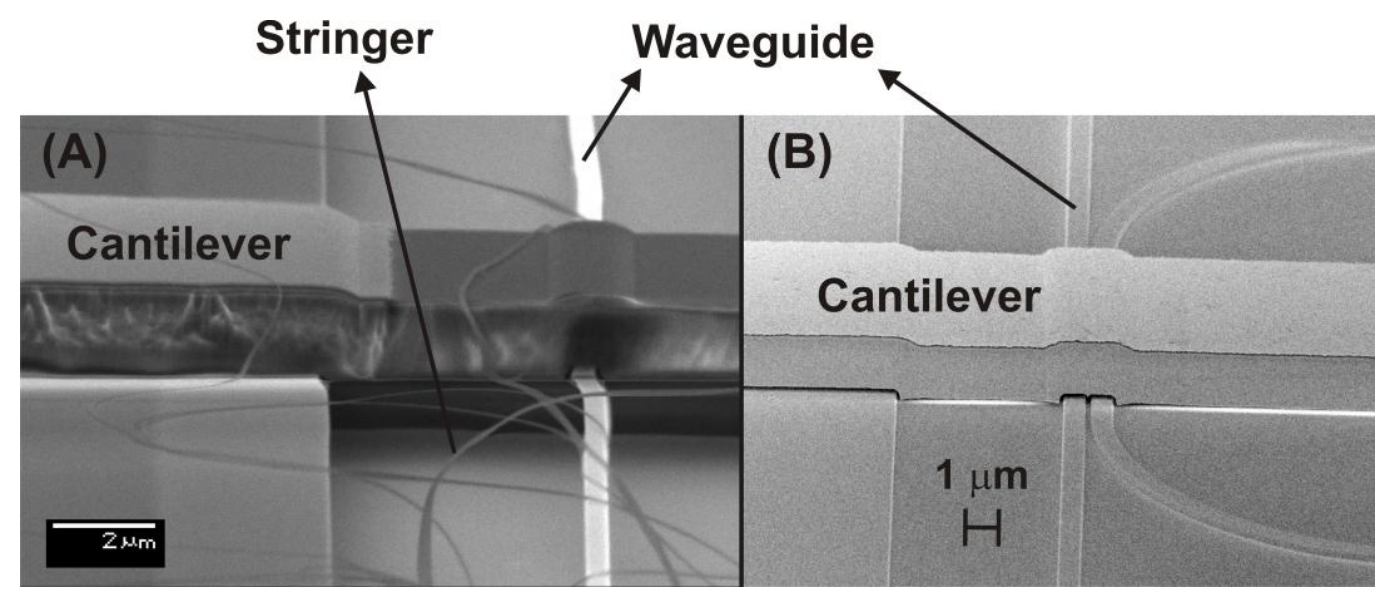

Figure 4.8. An integrated device (A) with unwanted stringers around the device, and (B) without stringers using the sonification technique described in section 4.4.2.4.

\subsubsection{Stringer formation}

In order to study the formation of stringers, Si nanoridges are fabricated using the technology described in [20]. The ridges are fabricated on a $100 \mathrm{~mm}$, p-type Si <110> wafer by contact print lithography. The height of the ridge is $150 \mathrm{~nm}$ and its width is 4 $\mu \mathrm{m}$. Figure 4.9A shows a top view of the nanoridges and Figure $4.9 \mathrm{~B}$ shows an oblique view of one of the ridges. The ridges have vertical sidewalls.

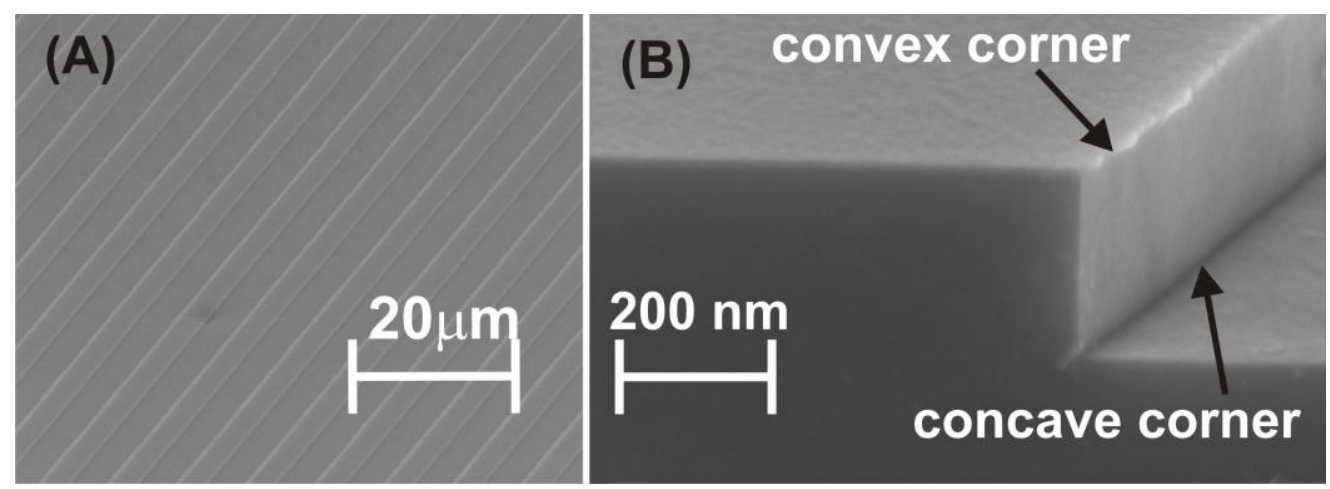

Figure 4.9. Top view (A) of silicon nanoridges and (B) oblique view of one of the nanoridges.

Subsequently, a $1.0 \mu \mathrm{m}$ thick $\mathrm{SiN}_{x}$ layer is conformally deposited on top of the ridges using LPCVD method. One of the important features of this deposition is the circular broadening $(\rho)$ of the deposited layer around a sharp convex corner while the concave corner remains sharp [21]. When the thickness of the $\mathrm{SiN}_{x}$ layer is less than or equal to the step height of the ridge, the concave corner angle $(\alpha)$ between the planar and curved surface of the deposited layer is $90^{\circ}$, but when it is more, the angle becomes obtuse. Figure 4.10A shows how the angle $\alpha$ changes from $90^{\circ}$ into obtuse with increasing thickness of the deposited layer and Figure 4.10B shows an SEM image of a $1.0 \mu \mathrm{m}$ conformal layer deposited on the nanoridge resembling layer ' 5 ' in Figure 4.10A. 
Chapter 4. Micro-cantilever integrated micro-ring resonator: Fabrication

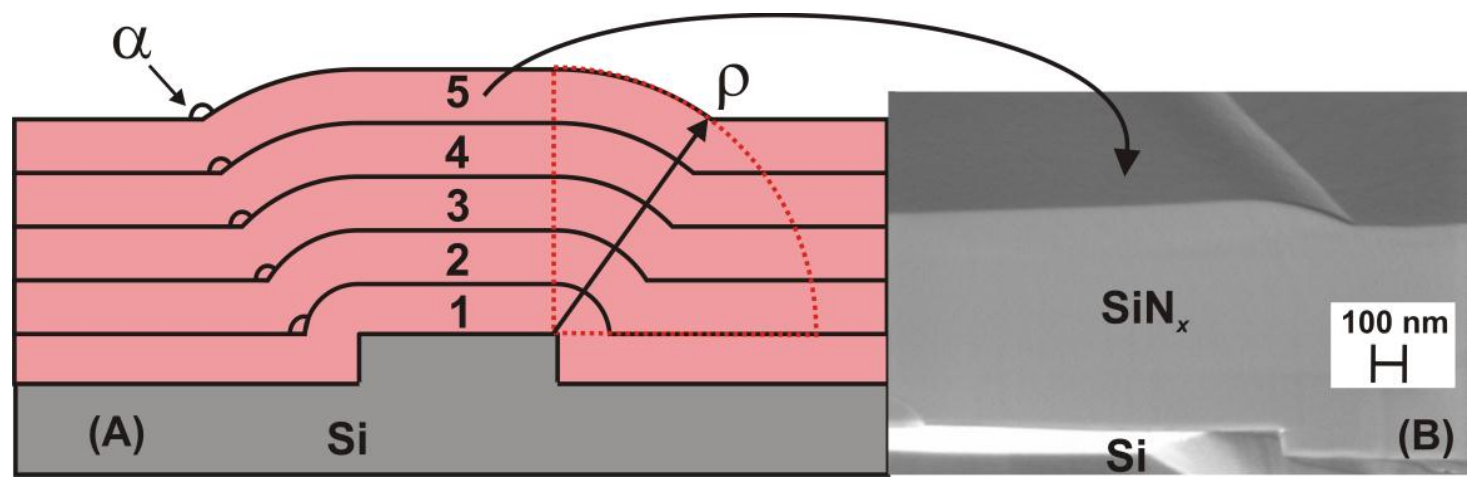

Figure 4.10. (A) Dependence of angle ' $\alpha$ ' to the thickness of the deposited layer and (B) SEM image of 1.0 um conformal layer deposited on top of a $150 \mathrm{~nm}$ step.

Subsequently, directional $\operatorname{SiN}_{x}$ etching is done by RIE (Elektrotech PF 340 at

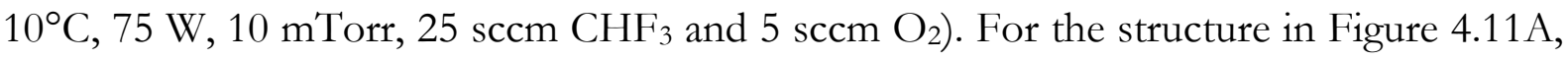
performing a directional $\mathrm{SiN}_{x}$ etch, which exactly stops at the planar $\mathrm{Si}$ interface, leaves stringers at the corners of the Si ridges (Figure 4.11B) and when $\alpha$ is $90^{\circ}$, the aspect ratio (height to width) of the formed stringers is more than 1. Edge lithography [22], also known as spacer technology [23], utilises the stringers having such aspect ratios for fabrication of nano structures. As the thickness of the $\mathrm{SiN}_{x}$ layer increases compared to the step height, $\alpha$ becomes obtuse and the aspect ratio tends to be smaller than 1 . Thus the aspect ratio of the remaining stringer in a directional etching step is primarily dependent on the thickness of the deposited (conformal) layer with respect to the step height.

Figure $4.10 \mathrm{~B}$
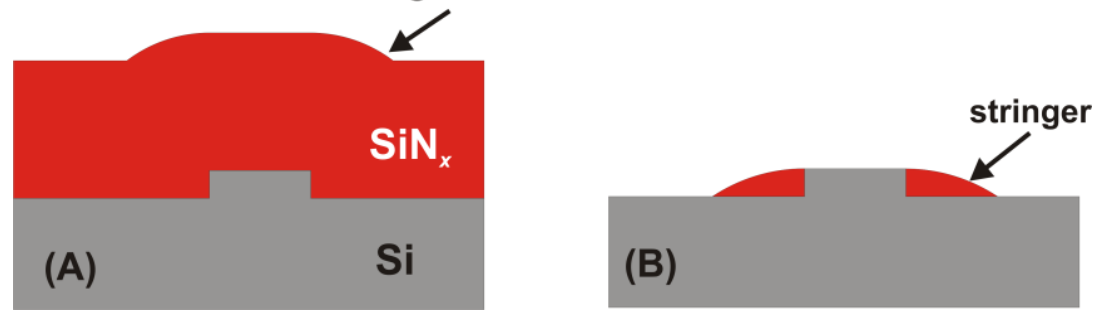

Figure 4.11 Analysing the shape of stringers for $(A)$ conformal coating on a step with a vertical sidewall and (B) a subsequent directional etching.

In order to analyse the shape of the stringers after a directional etching step (Figure 4.11B), the wafers are first etched in 1\% HF to remove the native oxide and in $25 \% \mathrm{KOH}$ solution at $75^{\circ} \mathrm{C}$ for 1 minute to etch some Si. The etch rate for $\mathrm{Si}<110>$ in $25 \% \mathrm{KOH}$ at $75^{\circ} \mathrm{C}$ is $1.4 \mu \mathrm{m} / \mathrm{min}$, but $\mathrm{SiN}_{x}$ is highly resistant to this solution $(1 \mathrm{~nm} / \mathrm{hr})$ and stays practically intact. The $\mathrm{KOH}$ etching clearly reveals the stringer to be in partialquadrant shape having a lateral width of $367 \mathrm{~nm}$ and a height of $135 \mathrm{~nm}$ (Figure 4.12). 


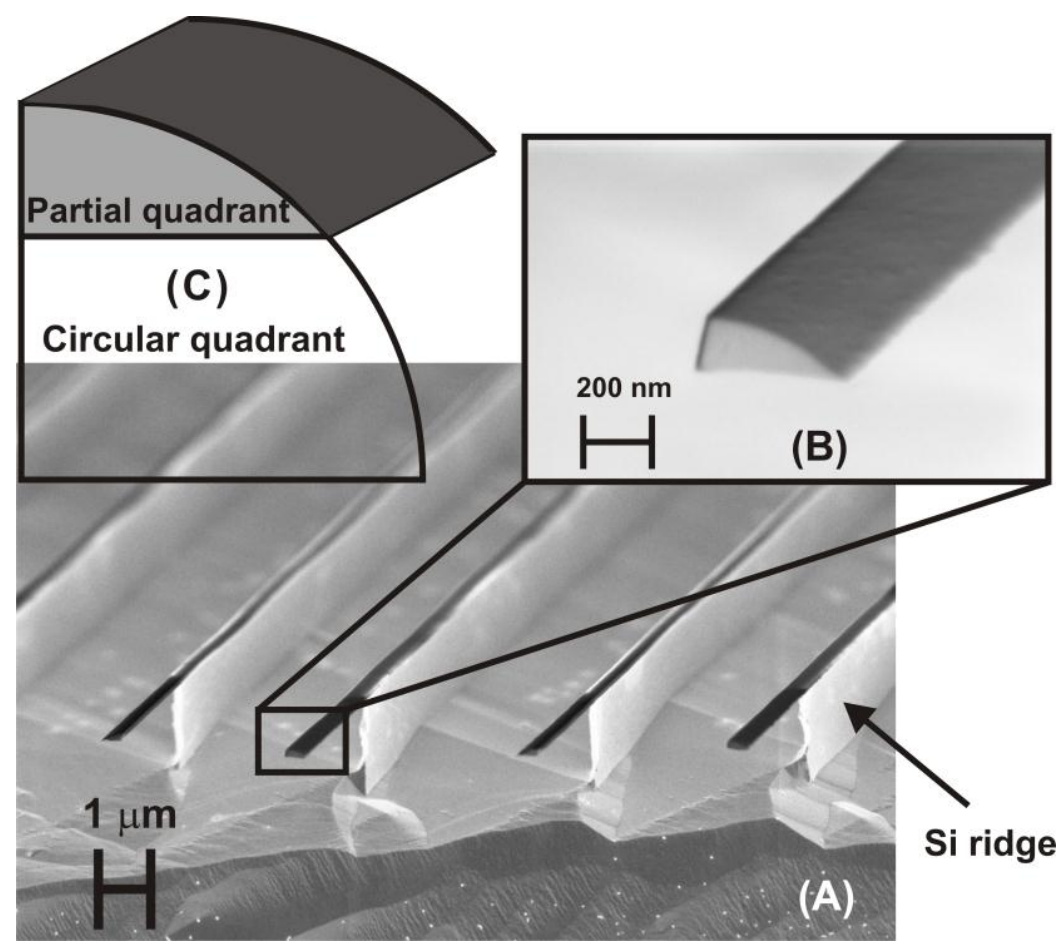

Figure 4.12. Stringers on top of $<110>$ silicon nanoridges after $\mathrm{KOH}$ etching.

Besides directional plasma etching, isotropic wet etching also leaves stringers at the corners, although the shape differs (Figure 4.13). Corner lithography [24] utilises this effect, to constitute the structural material or the masking material for the fabrication of various intriguing three-dimensional nano devices.

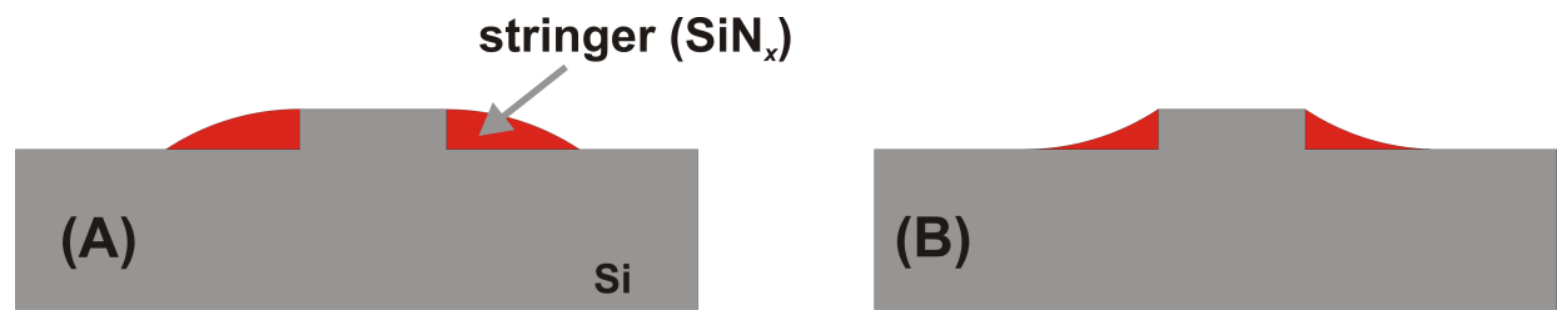

Figure 4.13. Stringers after (A) directional plasma etch (B) isotropic wet etch.

\subsubsection{Stringer elimination methods}

Even though stringers are utilised for fabricating nanostructures in edge and corner lithography, they are highly undesired in devices fabricated for a plethora of other applications. Hence stringer elimination methods play a vital role in the successful fabrication of many devices. For a better understanding of the stringer related problems, first a review on the various existing stringer elimination methods is described (Table 4-II). The methods consist of performing overetching, designing tapered sidewalls, and reshaping layers including the first new method. 
Chapter 4. Micro-cantilever integrated micro-ring resonator: Fabrication

Table 4-II. Advantages and disadvantages of various stringer elimination methods in micro and nanomachining.

\begin{tabular}{|c|c|c|}
\hline $\begin{array}{l}\text { Stringer elimination } \\
\text { methods }\end{array}$ & Advantages & Disadvantages \\
\hline $\begin{array}{l}\text { Directional } \\
\text { overetching }\end{array}$ & $\begin{array}{l}\text { No additional etch step. No } \\
\text { design change }\end{array}$ & Poor selectivity might harm underlying layers \\
\hline Isotropic overetching & $\begin{array}{l}\text { No additional etch step. No } \\
\text { design change }\end{array}$ & $\begin{array}{l}\text { Limited availability of etchants to perform the } \\
\text { selective task }\end{array}$ \\
\hline Tapered sidewall & No additional etch step & $\begin{array}{l}\text { Lowers the resolution and needs redesign of } \\
\text { the waveguide }\end{array}$ \\
\hline Double lithography & Local removal of stringers & $\begin{array}{c}\text { Extra lithography step required and possible } \\
\text { misalignment }\end{array}$ \\
\hline Planarization & No design change & $\begin{array}{l}\text { Selective stop close to the PL-SL interface } \\
\text { might be problematic }\end{array}$ \\
\hline
\end{tabular}

\subsubsection{Overetching procedure}

For processes having a high selectivity between the etched and the etch-stop layers, one method to eliminate stringers is by performing overetching. This applies to both isotropic and anisotropic etching. But, for the fabrication process described in section 4.3.2, if the etch selectivity is insufficient, performing an overetching might attack the underlying protective layer. Subsequently, it etches the waveguides along with the stringers. This can result in completely destroying the silicon waveguides or introducing roughness to their surface. Furthermore, a destroyed PL causes the lower oxide cladding layer to be exposed to BHF in the SLE step, resulting in the collapse of the waveguides and ring resonators onto the handle wafer.

Figure 4.14 shows the effect of performing overetching during the selective etching of a non-uniform $\mathrm{SiN}_{x}$ layer on top of a thin TEOS oxide layer (i.e., during step 'd' of Figure 4.3). The measured ' $3 \sigma$ ' for the cantilever device layer $\left(\mathrm{SiN}_{x}\right)$ is $50 \mathrm{~nm}$. Due to this, at some points of the wafer, the $\mathrm{SiN}_{x}$ thickness is less than the designed value whereas on other points it is above the designed value. This means that RIE overetching has to be done to completely remove $1.1 \mu \mathrm{m} \mathrm{SiN}$ from all over the wafer. The poor RIE selectivity limits the overetching to be less than 5 minutes for a SL thickness (tSL) of 196 $\mathrm{nm}(196 / 35 \sim 5.5)$. If a longer overetching time is used, the PL layer is under attack which finally results in destroying the ring resonator and its access waveguides. The collapse of waveguide structures is schematically presented in Figure 4.14-1d and an SEM 
image of it is shown in Figure 4.15A. Furthermore, the $\mathrm{SiN}_{x}$ cantilever layer has to be chosen sufficiently thick to prevent optical loss caused by the electrode. And so, the absolute non-uniformity is quite big ( $\pm 50 \mathrm{~nm}$ ), which is causing an etch delay across the wafer of several minutes. By carefully checking the thickness and non-uniformity of both layers with dummy wafers and by analyzing the loading effect, it was possible to successfully etch through a $1.1 \mu \mathrm{m} \mathrm{SiN}_{x}$ layer on top of a $196 \mathrm{~nm}$ SL. However, in this case the stringers were not completely removed by sufficient overetching (see Figure 4.15B), but $\operatorname{SiN}_{x}$ (cantilever) etching has been stopped when it has reached the planar TEOS-SiN $x$ (cantilever) interface, all over the wafer. The stringers, that remained due to etch delay caused by layer non-uniformities, were successfully removed by an ultrasonic cleaning technique as described in section 4.4.2.4.

(1)

(2)

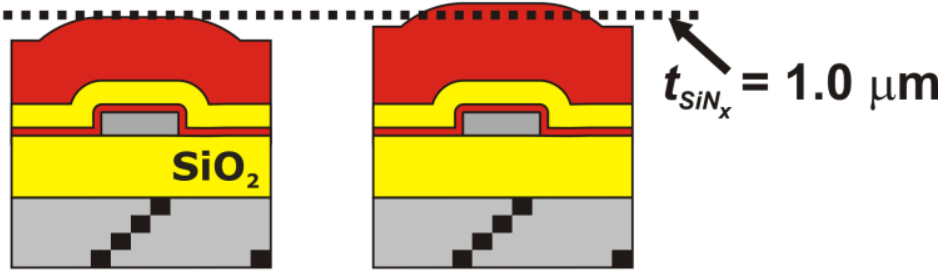

Various layers deposited on the SOI wafer

(step 'b' of Figure 4.3)

(b)
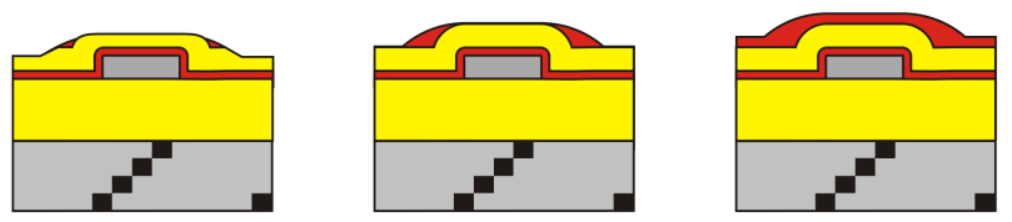

Etching of $\mathrm{SiN}_{x}$ cantilever layer

(c)
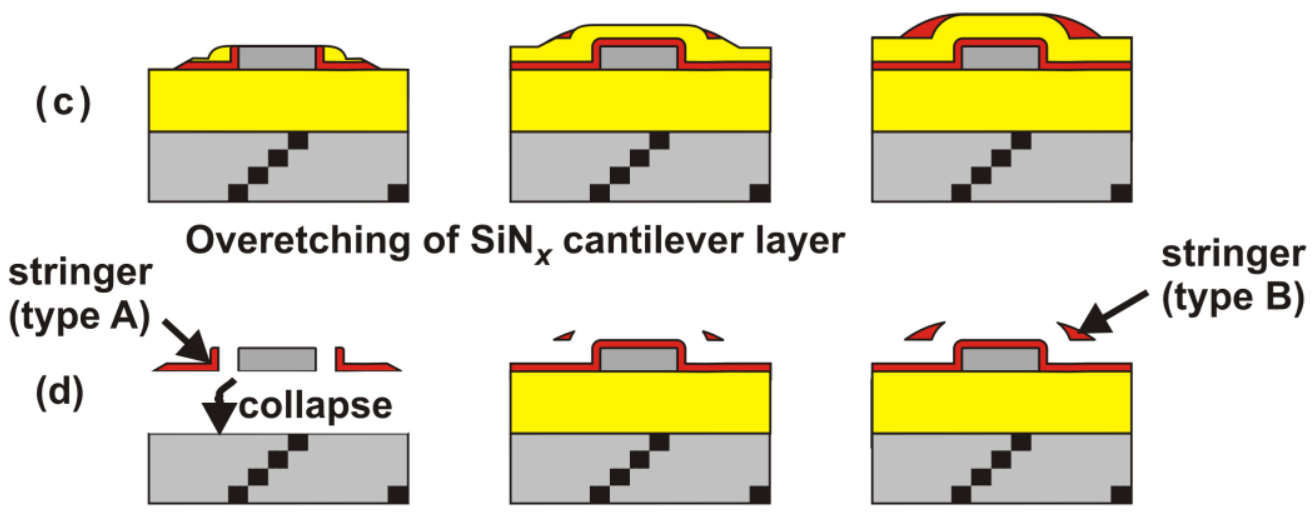

After sacrificial layer etching

Figure 4.14. Thickness variation of $S i N_{x}$ layer on the etching of a thick $S i N_{x}$ from a thin TEOS layer for (1) $t_{\mathrm{SiN} x}=0.95 \mu \mathrm{m}$, (2) $t_{\mathrm{SiN} x}=1.0 \mu \mathrm{m}$ and (3) $t_{\mathrm{SiN} x}=1.05 \mu \mathrm{m}$. 
Chapter 4. Micro-cantilever integrated micro-ring resonator: Fabrication

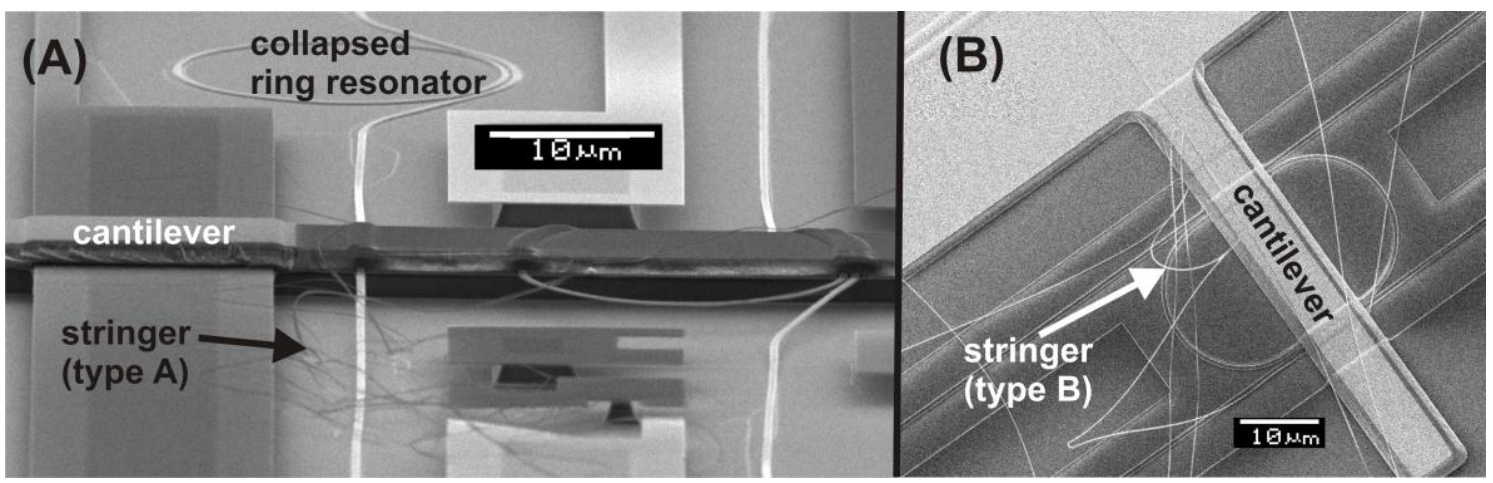

Figure 4.15. SEM images showing (A) the ring resonator and access waveguides collapsed by overetching of the SL and PL layer during the RIE of SiNx cantilever layer and (B) the stringers released after SLE, but still attached to the cantilever, when the overetching is not performed.

Using SF$_{6}$ based RIE (Adixen AMS100SE) [12], which has a higher etch selectivity of $\mathrm{SiN}_{x}$ over TEOS, is investigated as well. But this resulted in broken waveguides all over the wafer. It is believed that the silicon-rich particles in $\mathrm{SiN}_{x}$ are etched faster than silicon-poor areas, introducing pinholes into the $\mathrm{SiN}_{x}$ layer. Through these pinholes, the sacrificial and protective layers are etched by $\mathrm{SF}_{6}$ plasma, resulting in an attack of the underlying Si waveguides. During the subsequent SLE step, BHF passes through these pinholes and introduces an undercut in the oxide cladding layer around the pinholes. Figure 4.16A shows an optical microscopic (OM) image and Figure 4.16B an SEM image of the pinholes and broken Si waveguides introduced by $\mathrm{SF}_{6}$ plasma etching. Instead of the silicon-rich $\mathrm{SiN}_{x}$ layer, selecting a stoichiometric $\mathrm{Si}_{3} \mathrm{~N}_{4}$ layer for the cantilever might resolve this problem, but in that case stress in $\mathrm{Si}_{3} \mathrm{~N}_{4}$ is an issue to be considered. In total, the above possible techniques were disregarded to resolve the stringer formation and it was opted to use an ultrasonic technique instead.

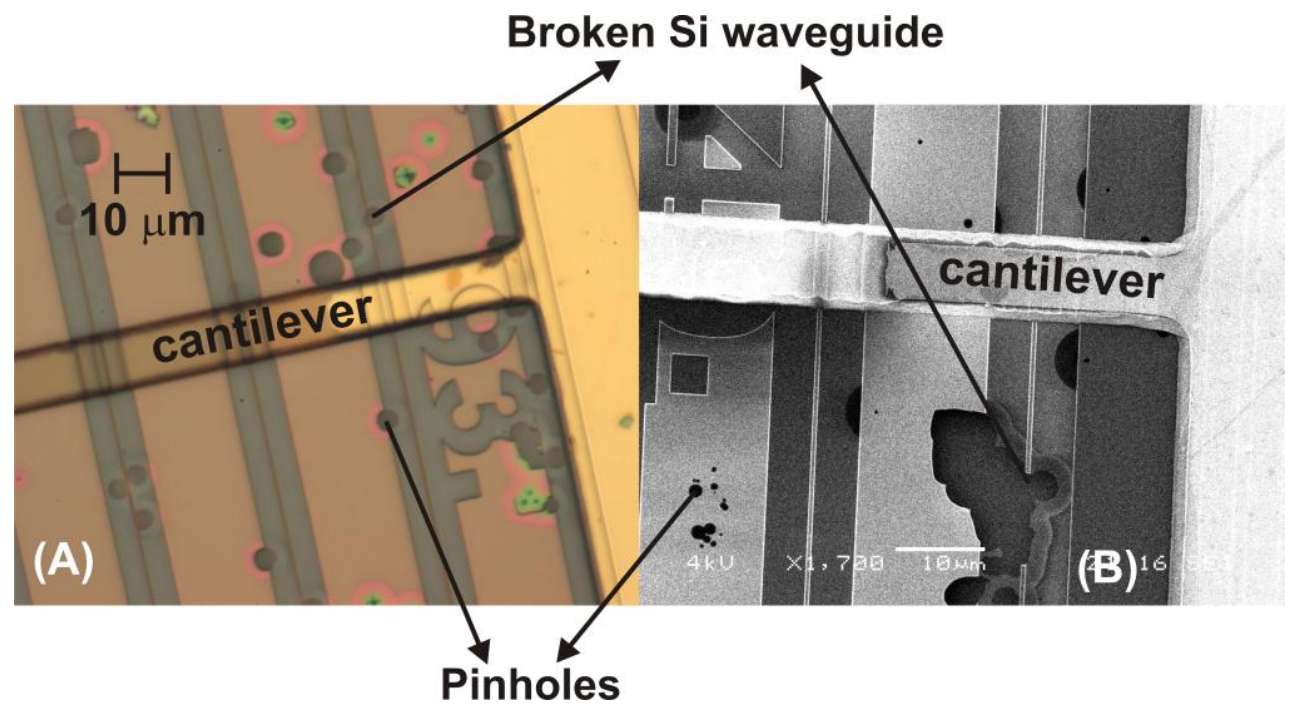

Figure 4.16. (A) OM and (B) SEM images of the pinholes and broken Si waveguides after SF 6 plasma etching. 


\subsubsection{Tapered sidewall procedure}

In some cases the stepped surface needs not to be perfectly at right angles to the wafer. In such cases, designing a tapered sidewall will remove the stringer comparatively faster than in the case of a vertical sidewall [17]. Figure 4.17 shows the concept of the tapered sidewall procedure. However, in the case of optical designs, selecting tapered waveguide sidewalls will change the properties and performance of the waveguide requiring a re-design, which was found to be undesired.

(A')

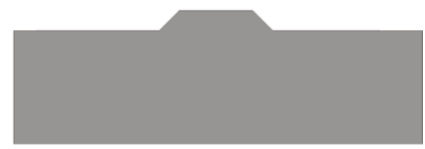

(B')

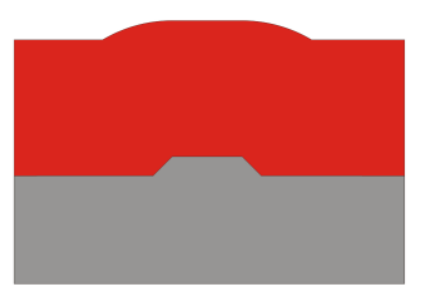

stringer

(C')
(1)

(A)

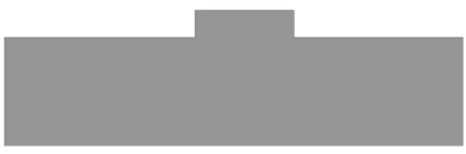

(B)

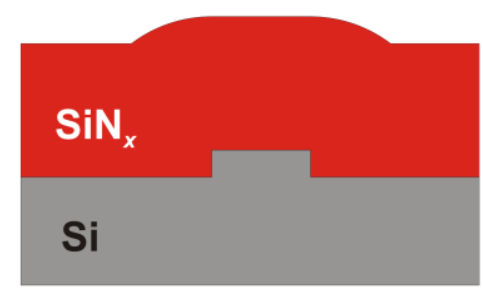

(2)

(C)

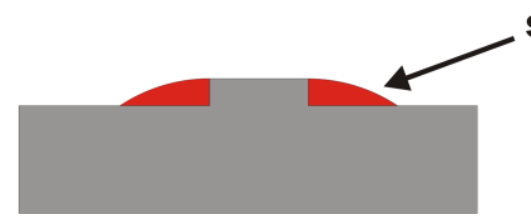

Figure 4.17. Effect of (1) vertical and (2) tapered sidewall on the stringer formation.

\subsubsection{Reshaping procedure}

Planarization of layers by chemical mechanical polishing (CMP) has been a technique widely used for eliminating the difficulties arising due to non-planar topography. This technique is effectively used to eliminate stringers in [18] where the SL is planarized prior to the subsequent deposition. The drawback of this method is, though, that selective stopping during the planarization (of a much thicker sacrificial layer) close to the PL-SL interface to create a well-defined gap is problematic and, therefore, it has been abandoned.

To circumvent the inconvenience of altering the optical performance of waveguides due to tapered sidewalls, an alternative approach is treated next. The idea is built on the use of residue in corners or edges of features (Figure 4.12) to reshape the SL. It is presented next with the aid of the various schemes displayed in Figure 4.18. 
Chapter 4. Micro-cantilever integrated micro-ring resonator: Fabrication

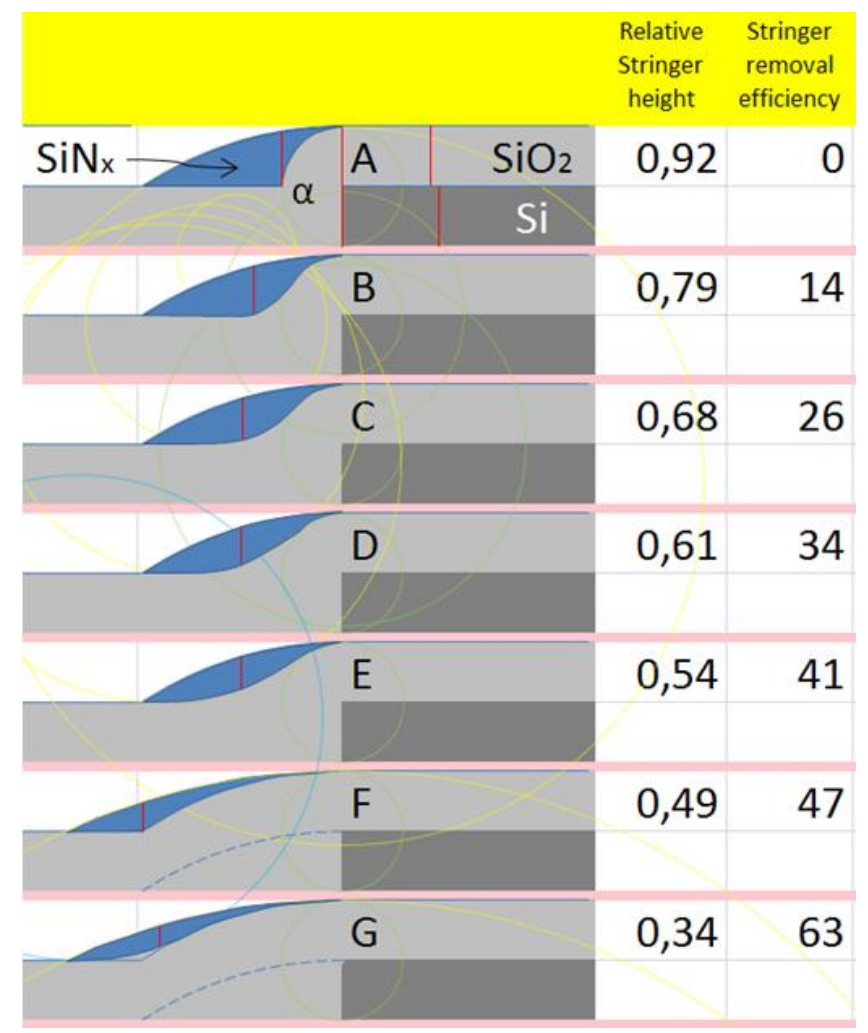

Figure 4.18. Stringer shape optimisation for improved removal efficiency.

Now, it is concentrated on Figure 4.18. In all schemes, a few conditions are preserved. In the first place, the (silicon) step height is fixed at $200 \mathrm{~nm}$. On top of this, the (silicon oxide) SL is deposited and reshaped. Then, the $1000 \mathrm{~nm}$ (silicon nitride) structural layer is uniformly deposited and directionally etched back using RIE. So, all steps are fixed except the shaping of the SL is varied in order to minimize the stringer residue. Scheme "A" shows the usual "shark fin-shape" after a simple conformal deposition. The stringer has three convex corners, from which " $\alpha$ " is the most disturbing one, because at this position the stringer height is at its maximum value $(0.92$ times the $\mathrm{Si}$ step height). Remember that the stringer is caused by a uniform coating with LPCVD nitride and a subsequent directional RIE step. To get rid of this sharp corner, in "B", first $400 \mathrm{~nm}$ SL is deposited and then isotropically etched back, leaving the requested $200 \mathrm{~nm}$ SL thickness. However, due to this extra back etch, the concave corner " $\alpha$ " is rounded, which reduces the step height of the stringer locally down to 0.79 times the Si step height. This will relax the time needed to remove the complete stringer by $14 \%$; the stringer removal efficiency. The schemes "C" till " $\mathrm{E}$ ", further improves the removal efficiency from:

26\% (C: $600 \mathrm{~nm}$ deposition and $400 \mathrm{~nm}$ etch), to

34\% (D: $800 \mathrm{~nm}$ deposition and $600 \mathrm{~nm}$ etch), to

41\% (E: $1000 \mathrm{~nm}$ deposition and $800 \mathrm{~nm}$ etch). 
Clearly, the stringer removal efficiency improves with thicker deposited layers and more etch back, but suffers from increasing inaccuracy in the SL thickness after these combined conformal deposition and isotropic etching steps. It looks like the "cat eyeshaped" profile is causing this limitation. For this reason, the back etch is changed from isotropic into directional. Scheme F represents this situation, where $1000 \mathrm{~nm} \mathrm{SL}$ is deposited first and then $800 \mathrm{~nm}$ is etched back directionally, leaving again $200 \mathrm{~nm}$. If now the $1000 \mathrm{~nm}$ nitride layer is deposited and etched with RIE, the removal efficiency goes seemingly to $47 \%$. However, as shown in Figure 4.19, RIE of material conformally grown over sharp concave corners result in rounded shapes and this is clearly favourable since no additional conformal deposition with isotropic etch back to round off the corner $\alpha$ is needed (scheme G).

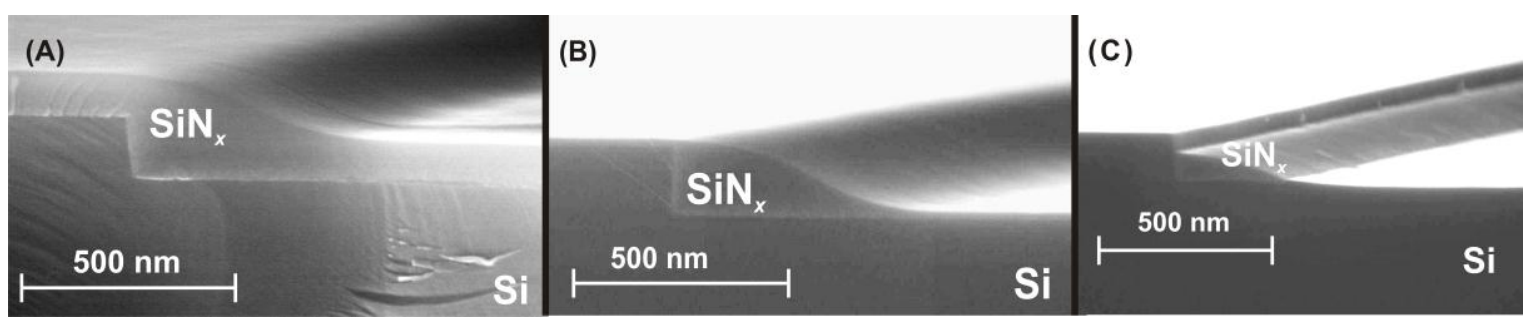

Figure 4.19. Material reshaping at the concave corners in directional RIE before reaching the Si.

This corner-rounding during RIE could be due to nitride redeposition or ion deflection from surface charging $[11,25]$. This insight would mean that it is allowed to adjust the original procedure of a single deposition step into a scheme, where $1000 \mathrm{~nm}$ TEOS is deposited first and then remove $800 \mathrm{~nm}$ of it using RIE. This scheme might be very effective in preventing stringer residue and reduces the stringer removal time by $63 \%$.

Even though the previous reshape procedure seems to be very promising and easily to integrate with any existing micro/nano technology, in the coming section the pros and cons of another technique is shown, which has resulted in a successful functioning of stringer-free optical devices.

\subsubsection{Stringer elimination using surface micromachining and sonification}

In many cases the aforementioned methods are sufficient to remove the unwanted stringers, but the methods are inappropriate in the design we have followed mainly due to the lack of etch selectivity. Therefore, a simple alternative procedure to effectively remove stringers is presented next, i.e., the wafer is immersed in an ultrasonic bath. The method neither requires extra lithography steps nor changes the design of the process. However, as the technique relies on released stringers, a SLE of the stringer prior to ultrasonic 
Chapter 4. Micro-cantilever integrated micro-ring resonator: Fabrication

treatment is required. Next, a brief description of the effect of ultrasound on stringers is presented.

Ultrasound - or sonification - is a method to remove particulate contamination on surfaces [26-27] for micromachining of both hard and brittle materials [28-29]. The technique is commonly used in chemistry laboratories and semiconductor industry, and many effects have been considered to explain its efficiency and limitations. Sonification is the process in which vibration in a liquid causes acoustic cavitation; the formation, growth and implosion of bubbles near topographic surfaces. This generates shockwaves within the liquid, dislodging contaminants from a surface (UC: ultrasonic cleaning), localised erosion of the material due to impact of abrasive particles (USM: ultrasonic machining) or even fracture of slender structures. Evidently there exists an optimum; if the physical force applied for dislodging the particles is less than the adhesive force, the particles remain at the surface, but if the force is greater than what the structure can sustain, it leads to damage. Sonification is performed in (de-ionised) water or other lowviscous liquids in tanks with a piezoelectric transducer placed at the bottom. The transducer typically vibrates $>20 \mathrm{kHz}$ causing pressure waves to propagate through the tank. The process of ultrasonic cleaning is nicely described by Yin and Komvopoulos in 2010 [30]: "Each point along a wave oscillates between a pressure maximum (compression) and a pressure minimum (rarefaction). Cavitation bubbles form at sites where the pressure drops below the vapour pressure of the fluid, approaching the pressure minimum. These bubbles subsequently collapse as those sites transition from pressure minimum to the pressure maximum. Rapid flow of surrounding fluid to fill the void created by a collapsed bubble produces an intense shock wave. The cavitation energy transferred to the surface due to the collapse of many bubbles close to the substrate surface activates an intense scrubbing process, which is effective in removing contamination from the surface of devices". The sonification effect is mainly depending on the number of bubble implosions, which is typically enlarged by increasing parameters like temperature, transducer input power, or its frequency. Furthermore, the type of liquid (a low viscosity will encourage collapse) and the amount and type of dissolved gases in it (filling the bubble with its partial pressure and thus preventing a violent collapse) will have effect on the cavitation erosion [31]. It has been found that bubbles preferentially nucleate around strong irregularities - like the stringers - and the collapse may be even stronger in confined spaces - like the one underneath the stringer [27]. However, the selective abrasive effect of sonification on suspended slender structures is largely unknown and it should be understood that a careful study needs both sufficient experimental evidence and an in-depth fundamental model. Although important, it falls beyond the scope of the current research to study and verify this fundamental model. The dependence of damage on structure size has been studied in megasonic cleaning (sonification around $1 \mathrm{MHz}$ ) [31-32]. It was found that 
damage in nano-sized ridges is caused mainly by asymmetric collapse of bubbles during which a micrometer-sized jet impinges on the wafer, generating a high shear stress on the wafer surface. This asymmetry in the bubble is created when it comes close to a surface. More important, the damage of different line widths was studied in [31-32] as well and it was found that smaller line widths incurred substantial more damage. For example, structures having a width of $60 \mathrm{~nm}$ were 10 times easier to fracture than those having a width of $100 \mathrm{~nm}$ [32]. Moreover, the surveys also indicated that the manufacturing conditions of the structures could significantly impact the level of damage. This suggests that megasonic cleaning - and most likely ultrasonic cleaning in general - results from damaging weak spots in structures (e.g. variations in line-width, grain boundaries, or particles) that have seen high-energy cavitation events. They further found evidence that, while the number of damage events can be changed, the damage per event remains the same [33].

Now an analysis is carried out on the effect of stringers in the process described in section 4.3.2, having a SLE step to release the bimorph cantilevers. During SLE the stringers are released from the sides of the optical waveguide too and are loosely distributed all over the wafer. Since the stringers are still attached to the cantilevers they are not washed away during SLE. Figure 4.20A shows an OM image of a cantilever integrated with a photonic crystal (PC) waveguide and Figure 4.20B shows an SEM image of a cantilever integrated with a microring resonator. Both images show stringers distributed on top of the device practically destroying its mechanical as well as optical performance.
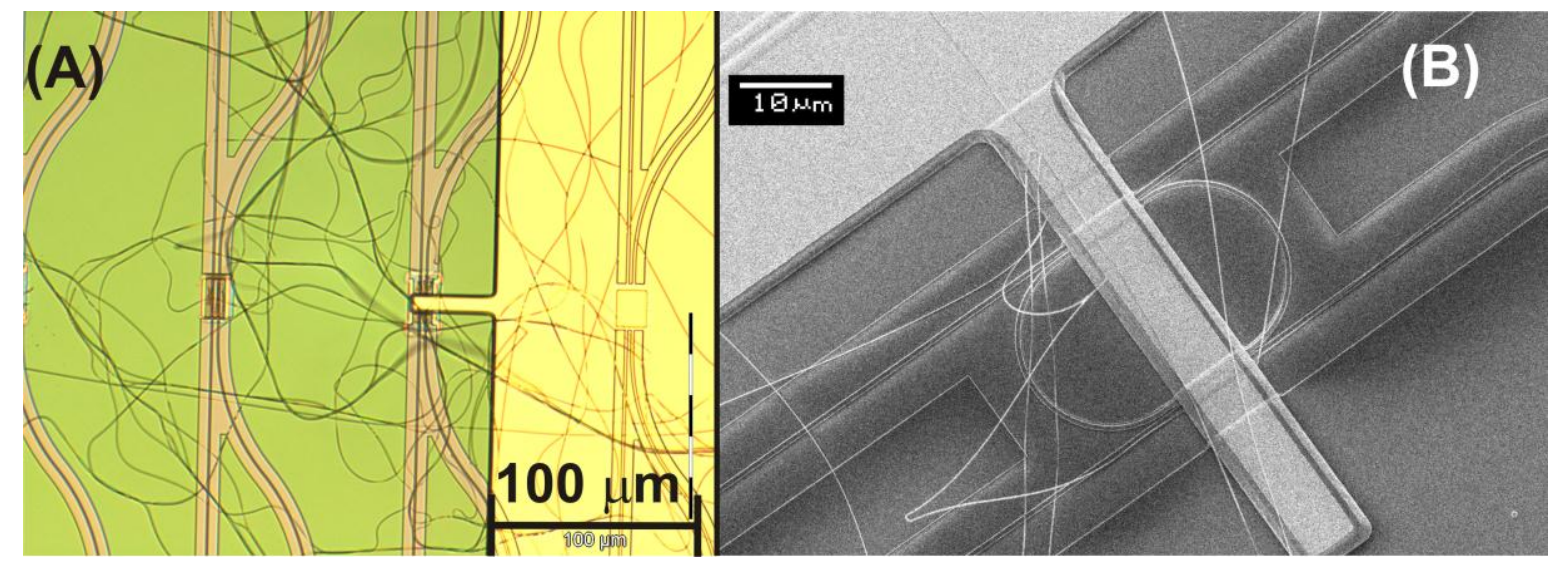

Figure 4.20. A cantilever integrated with (A) a photonic crystal waveguide and (B) a ring resonator, without ultrasonic cleaning. Stringers are loosely distributed all over.

The suggested method used for removing stringers utilises the acoustic waves produced in ultrasonic cleaning [26-27] to mechanically agitate the released stringers and thereby to break them off from the cantilevers. For this, the SLE is divided into three 
steps viz, SLE-initiation, ultrasonic stringer removal and SLE-completion. The stringers produced in this process are approximately $6 \mathrm{~mm}$ long, $500 \mathrm{~nm}$ wide and $100 \mathrm{~nm}$ high and the width of the cantilevers is $10 \mu \mathrm{m}$. In the short SLE-initiation step only the stringers are released but the cantilevers are still mechanically fixed to the wafer to prevent damage. Followed by this an ultrasonic wafer cleaning is performed (VWRUSC600D; $132 \mathrm{kHz}, 25^{\circ} \mathrm{C}, 15 \mathrm{~min}$ at $120 \mathrm{~W}$ ). The cleaner has a tank capacity of 5.4 litres and is filled with 4.7 litres of deionised water. The wafer was placed in a metallic beaker containing deionised water and the beaker was placed inside the tank. In this step the released stringers are mechanically agitated by the ultrasonic waves and detached from the cantilevers. Care must be taken that in between the SLE-initiation step and the ultrasonic stringer removal step, any wafer drying step is avoided. This would cause the released stringers to stick onto the wafer, making ultrasonic cleaning ineffective. After the ultrasonic stringer removal step, the processing is continued with the SLE-completion step followed by a freeze drying step to release the cantilevers. Again, care should be taken to prevent any wafer drying before freeze drying as it will render the cantilevers stuck to the underlying surface. Figure 4.21A shows an OM image and Figure 4.21B shows an SEM image of the integrated device after including the intermediate ultrasonic stringer removal step. The stringers are completely removed from the wafer and the cantilevers are released from the substrate.

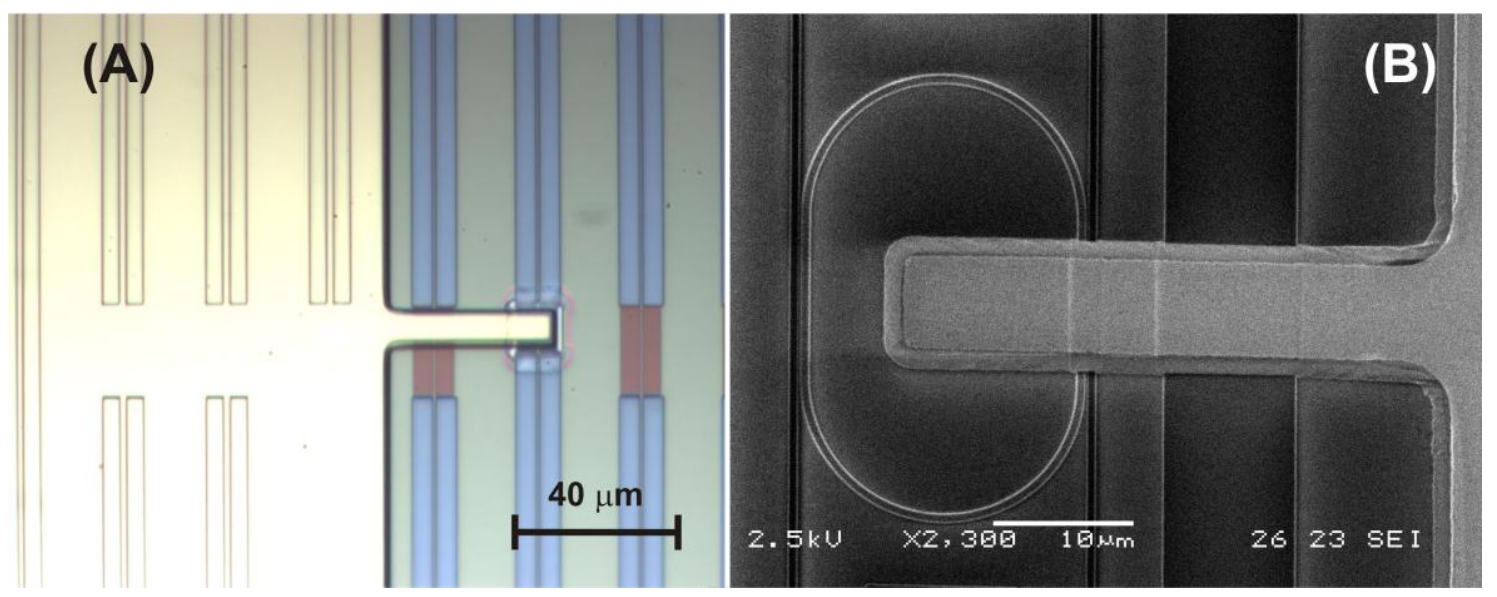

Figure 4.21. A cantilever integrated with (A) a PC waveguide and (B) a microring resonator. Ultrasonic cleaning is included during SLE to remove the stringers.

\subsection{Conclusions}

A technology for integrating MEMS with silicon integrated optical devices has been reported using surface micromachining techniques. A micro-cantilever integrated racetrack ring resonator has been fabricated successfully on SOI wafer. Various fabrication-related issues are discussed on which an important one is related with the 
formation of 'stringers'. A detailed analysis on stringer formation and a review on various methods used in micromachining for stringer elimination are presented. Two novel techniques for successful stringer removal are presented. The first method introduces additional steps to reshape an intermediate layer such that the stringer is much easier to be removed up to a maximum removal efficiency of $60 \%$. A second efficient alternative method to completely remove stringers (i.e. 100\% removal efficiency) by mechanical agitation by ultrasonic cleaning is developed, but the procedure might fracture slender devices as well and one should tune the ultrasonic conditions properly.

\subsection{References}

[1] D. G. Rabus, et al., "Ring resonator lasers using passive waveguides and integrated semiconductor optical amplifiers," IEEE Journal on Selected Topics in Quantum Electronics, vol. 13, pp. 1249-1256, 2007.

[2] G. N. Nielson, et al., "Integrated wavelength-selective optical MEMS switching using ring resonator filters," IEEE Photonics Technology Letters, vol. 17, pp. 1190-1192, 2005.

[3] P. T. Rakich, et al., "Ultrawide tuning of photonic microcavities via evanescent field perturbation," Optics Letters, vol. 31, pp. 1241-1243, 2006.

[4] S. T. Chu, et al., "Cascaded microring resonators for crosstalk reduction and spectrum cleanup in Add-Drop filters," IEEE Photonics Technology Letters, vol. 11, pp. 1423-1425, 1999.

[5] A. Yalcin, et al., "Optical sensing of biomolecules using microring resonators," IEEE Journal on Selected Topics in Quantum Electronics, vol. 12, pp. 148-154, 2006.

[6] E. J. Klein, "Densely integrated microringresonator based components for fiber-to-the home applications," PhD thesis, University of Twente, 2007.

[7] L. J. Kauppinen, "Compact integrated optical devices for optical sensor and switching applications," PhD Thesis, University of Twente, 2010.

[8] D. Taillaert, et al., "Grating couplers for coupling between optical fibers and nanophotonic waveguides," The Japan Society of Applied Physics, vol. 45, pp. 6071-6077, 2006.

[9] P. Dumon, et al., "Towards foundry approach for silicon photonics: silicon photonics platform ePIXfab," Electronics Letters vol. 45, pp. 581-582, 2009.

[10] J. G. E. Gardeniers, et al., "LPCVD silicon-rich silicon nitride films for applications in micromechanics, studied with statistical experimental design," Journal of V acuum Science and Technology A: Vacuum, Surfaces and Films, vol. 14, pp. 2879-2892, 1996.

[11] H. Jansen, et al., "A survey on the reactive ion etching of silicon in microtechnology," $J$. Micromech. Microeng., vol. 6, pp. 14-28, 1996.

[12] H. V. Jansen, et al., "Black silicon method X: A review on high speed and selective plasma etching of silicon with profile control: An in-depth comparison between Bosch and cryostat DRIE processes as a roadmap to next generation equipment," J. Micromech. Microeng., vol. 19, p. 033001, 2009.

[13] R. Legtenberg and H. A. C. Tilmans, "Electrostatically driven vacuum-encapsulated polysilicon resonators Part I. Design and fabrication," Sensors and Actuators A, vol. 45, pp. 57-66, 1994.

[14] G. E. Gimpelson, "Photoresist tapering process," United States Patent, 1987.

[15] T. Holmgaard, et al., "Dielectric-loaded plasmonic waveguide-ring resonators," Optics Express, vol. 17, pp. 2968-2975 2009.

[16] A. D. López, et al., "Two-dimensional MEMS array for maskless lithography and wavefront modulation," in Proc. SPIE, Smart Sensors, Actuators, and MEMS III Maspalomas, Gran Canaria, Spain 2007, pp. 65890S1-8. 
[17] A. S. Zoolfakar, et al., "The elimination of poly stringer in double poly AMS technology development," in ICSICT-2006: 8th International Conference on Solid-State and Integrated Circuit Technology, Shanghai, 2006.

[18] R. D. Nasby, et al., "Application of chemical-mechanical polishing to planarization of surface-micromachined devices," in Proc. IEEE Solid-State Sensor and Actuator Workshop, Hilton Head, SC, 1996, pp. 48-50.

[19] P. Jacob, "Poly-si extensions and etching residues as a reliability risk " Microsystem Technologies vol. 15, pp. 169-174, 2009.

[20] J. Haneveld, et al., "Nano-ridge fabrication by local oxidation of silicon edges with silicon nitride as a mask," J. Micromech. Microeng., vol. 16, pp. S24-S28, 2006.

[21] J. Schlote, et al., "Topological effects regarding trench structures covered with LPCVD and PECVD thin films," Surface and Coatings Technology, vol. 59, pp. 316-320, 1993.

[22] Y. Zhao, et al., "Sub-10 nm silicon ridge nanofabrication by advanced edge lithography for NIL applications," Microelectronic Engineering, vol. 86, pp. 832-835, 2009.

[23] D. C. Flanders and N. N. Efremow, "Generation of $<50 \mathrm{~nm}$ period gratings using edge defined techniques," Journal of vacuum Science and Technology B, vol. 1, pp. 1105-1108, 1983.

[24] J. W. Berenschot, et al., "3D-Nanomachining using corner lithography," in IEEE International Conference on Nano/Micro Engineered and Molecular Systems, NEMS, Sanya, China, 2008.

[25] T. C. Mele, et al., "Selective and anisotropic reactive ion etch of LPCVD silicon nitride with CHF3 based gases," Journal of vacuum Science and Technology B, vol. 2, pp. 684-687, 1984.

[26] A. A. Busnaina and F. Dai, "The Removal of Submicron Particles in Liquid-Based Cleaning," The Journal of Adhesion vol. 67, pp. 181-193, 1998.

[27] E. Maisonhaute, et al., "Surface acoustic cavitation understood via nanosecond electrochemistry. Part III: Shear stress in ultrasonic cleaning," Ultrasonics Sonochemistry, vol. 9, pp. 297-303, 2002.

[28] C. Zhang, et al., "Advances in micro ultrasonic assisted lapping of microstructures in hard-brittle materials: A brief review and outlook " International Journal of Machine Tools and Manufacture, vol. 45, pp. 881-890, 2005.

[29] T. B. Thoe, et al., " Review on ultrasonic machining," Internation Journal of Machine Tools Manufacturing, vol. 38, pp. 239-255, 1998.

[30] X. Yin and K. Komvopoulos, "Dynamic finite element analysis of failure in alternating phase-shift masks caused by megasonic cleaning," IEEE Transactions on components and packaging technologies, vol. 33, pp. 46 - 55, 2010.

[31] S. Helbig, et al., "Impact of MegaSonic process conditions on PRE and Sub-resolution assist feature damage," SPIE proceedings, vol. 7379, pp. 73790D1-10, 2008.

[32] F. Barbagini, et al., "Particle Removal Efficiency and Damage Analysis on Silicon Wafers after Megasonic Cleaning in Solvents," Journal of Adhesion Science and Technology, vol. 23, pp. 1709-1721, 2009.

[33] C. D. Marco, et al., "Damage clustering and damage-size distributions after megasonic cleaning," ECS Transactions, vol. 11, pp. 87-93, 2007. 


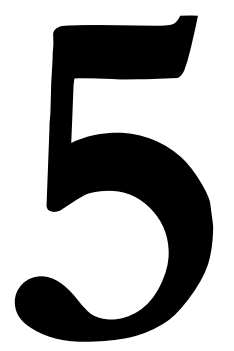

\section{Micro-cantilever integrated micro-ring resonator: Measurements}

This chapter presents the mechano-optical characterisation of micro-ring resonators with integrated micro-cantilevers. Using electrostatic actuation, moving the cantilevers deeper into this evanescent field, the propagation properties of the ring waveguide are modulated. A $122 \mathrm{pm}$ tuning range of the resonance wavelength of the optical ring resonator (in the optical C-band) without change in the optical quality factor, on application of $9 \mathrm{~V}$ to a $40 \mu \mathrm{m}$ long cantilever is demonstrated. Dynamic operation of the device with a $0-4 V$ square wave drive signal, showing a signal rise time of $16 \mu$ s and fall time of $4 \mu$ s is presented.

*Part of this chapter has been published as: L.J. Kauppinen, S.M.C. Abdulla, M. Dijkstra, M.J. de Boer, E. Berenschot, G.J.M. Krijnen, M. Pollnau and R.M. de Ridder, "Micromechanically tuned ring resonator in silicon on insulator", Optics Letters, Vol. 36, pp. 1047-1049, (2011).

*Part of this chapter is submitted for publication as: S.M.C. Abdulla, L.J. Kauppinen, M. Dijkstra, M.J. de Boer, E. Berenschot, H.V. Jansen, R.M. de Ridder and G.J.M. Krijnen, “Tuning a racetrack ring resonator by an integrated dielectric MEMS cantilever". 
Chapter 5. Micro-cantilever integrated micro-ring resonator: Measurements

\subsection{Introduction}

This chapter presents the mechano-optical characterisation of the micro-ring resonators with integrated micro-cantilevers. In order to fully understand and quantify the performance of the devices both static and dynamic mechanical characteristics of the electrostatically actuated micro cantilever are investigated and static wavelength tuning and dynamic optical switching of the integrated device are determined. Figure 5.1A shows a 3D schematic of the integrated device with its vertical fibre couplers and electrical contact probes and Figure $5.1 \mathrm{~B}$ shows the various ports of the micro-ring resonator (MRR). For the exact dimensions of the device see section 4.2.

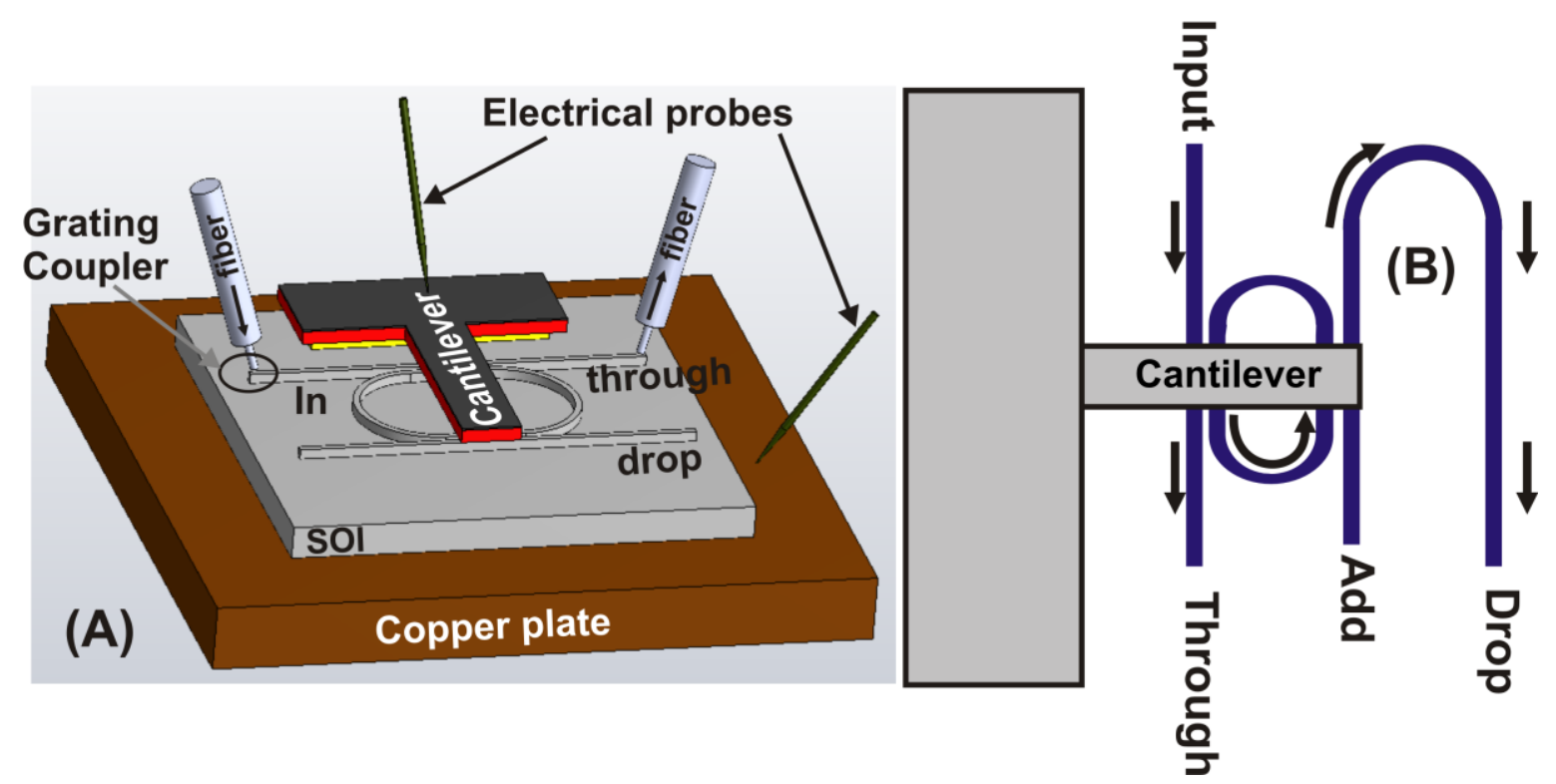

Figure 5.1. (A) Shows the 3-D schematic of the integrated system with its vertical fiber couplers and electrical contact probes (Image source: E. Berenschot, TST Group, University of Twente, The Netherlands) and (B) schematic of the various ports of the micro-ring resonator.

\subsection{Mechanical characterisation}

Static mechanical measurements are carried out at room temperature by white light interference microscopy (WLIM, Polytec MSA400) and dynamic measurements are done using the Laser Doppler Vibrometery (LDV, Polytec MSA400). Parameters like off-state deflection, pull-in voltage, cantilever resonance frequency, mechanical quality factor, reduction in resonance frequency by electrostatic spring softening and the time responses of the cantilevers are investigated. The off-state of the device refers to the situation in which there is no electrical signal, ie. cantilever is in the upper position, which in turn means the ring resonator is minimally affected by the cantilever. 


\subsubsection{Off-state deflection}

Off-state deflection of the micro-cantilevers (the vertical distance between the tip and the base) arising due to residual stress is an important parameter which has to be well controlled to optimize the performance of the device, as described in section 3.5.2. Residual stress in the deposited layers arises from various causes like dissimilar thermal expansion coefficients and deposition induced intrinsic stress [1]. After sacrificial etching and releasing, the bimorph micro-cantilevers curl upward, due to the tensile stress in the $\mathrm{Cr}$ and $\mathrm{Au}$ layers with respect to $\mathrm{SiN}_{x}$ layer. Figure 5.2A represents the off-state curvature of a $40 \mu \mathrm{m}$ long cantilever showing an upward tip-deflection of $94 \mathrm{~nm}$. Figure 5.2.B shows an optical microscopy image (OM) indicating the scan area used in this measurement. As discussed previously in section 4.3.4, the cantilever has a tapered geometry as a result of the processing specifics. Additionally the metal electrode is absent in the tapered areas of the cantilever. Hence the WLIM scanning area is restricted (e.g. at the position marked ' 3 ' in Figure 5.2B) to disregard areas where scattering effects by the nitride leads to ambiguous measurements. Thus, even though the cantilever covers both ring resonator coupling sections, the measurement in Figure 5.2A does not show the position of cantilever on top of the second coupling section (as marked ' 3 ' in Figure 5.2).

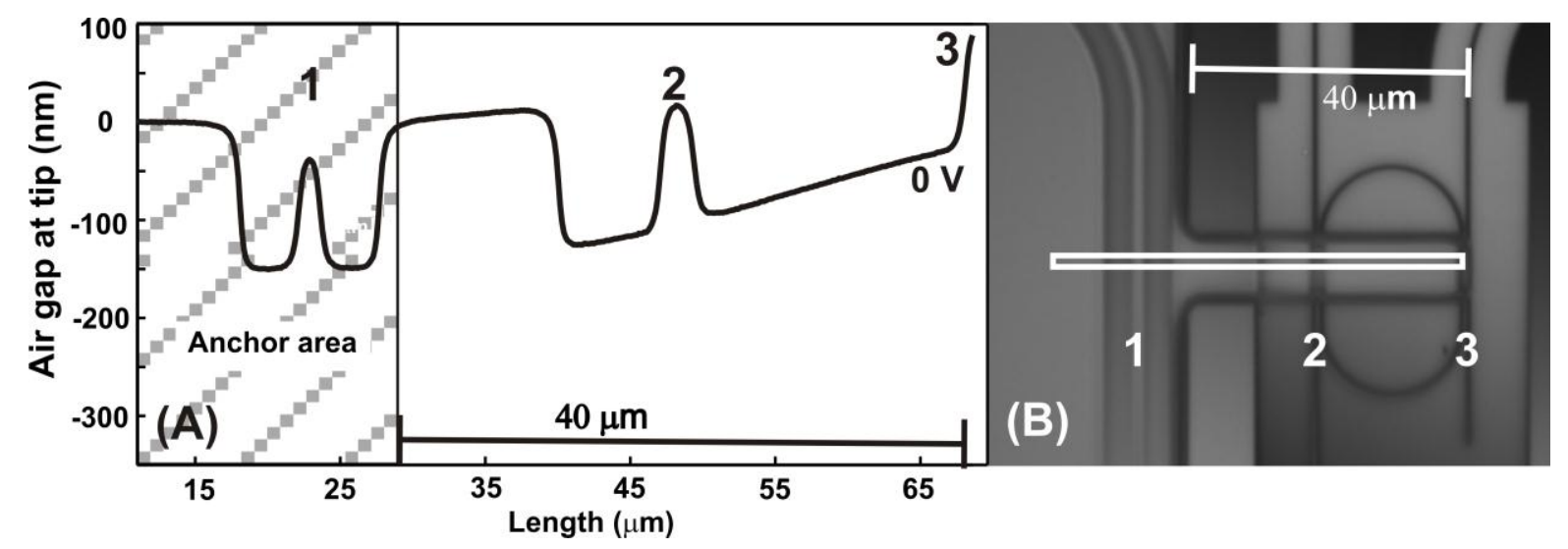

Figure 5.2. (A) Static deflection of a $40 \mu \mathrm{m}$ long cantilever at off-state $(O \mathrm{~V})$, measured by WLIM and (B) OM image of the integrated device with the white rectangle representing the WLIM scanning area.

Due to the curling of the cantilever, the air-gap between the cantilever and the ring resonator varies along the length. At the base, for cantilevers of all lengths, it is $196 \mathrm{~nm}$, which is the thickness of the sacrificial layer. At the tip of the bimorph it is given by the addition of the off-state tip deflection to the $196 \mathrm{~nm}$ sacrificial layer thickness. Thus a 40 $\mu \mathrm{m}$ long cantilever has approximately $290 \mathrm{~nm}(196+94)$ air-gap at its tip. As the microring resonators are fabricated by etching the silicon device layer down to the $\mathrm{SiO}_{2}$ cladding, the resulting stepped geometry is transferred into the subsequently conformally deposited layers. The hills and valleys observed in Figure 5.2A are due to the fact that the 
cantilever is deposited on top of the MRR. Figure 5.3 shows the measured cantilever offstate tip deflections plotted versus corresponding to the mask design length. Clearly, the deflection increases with the length of the beam.

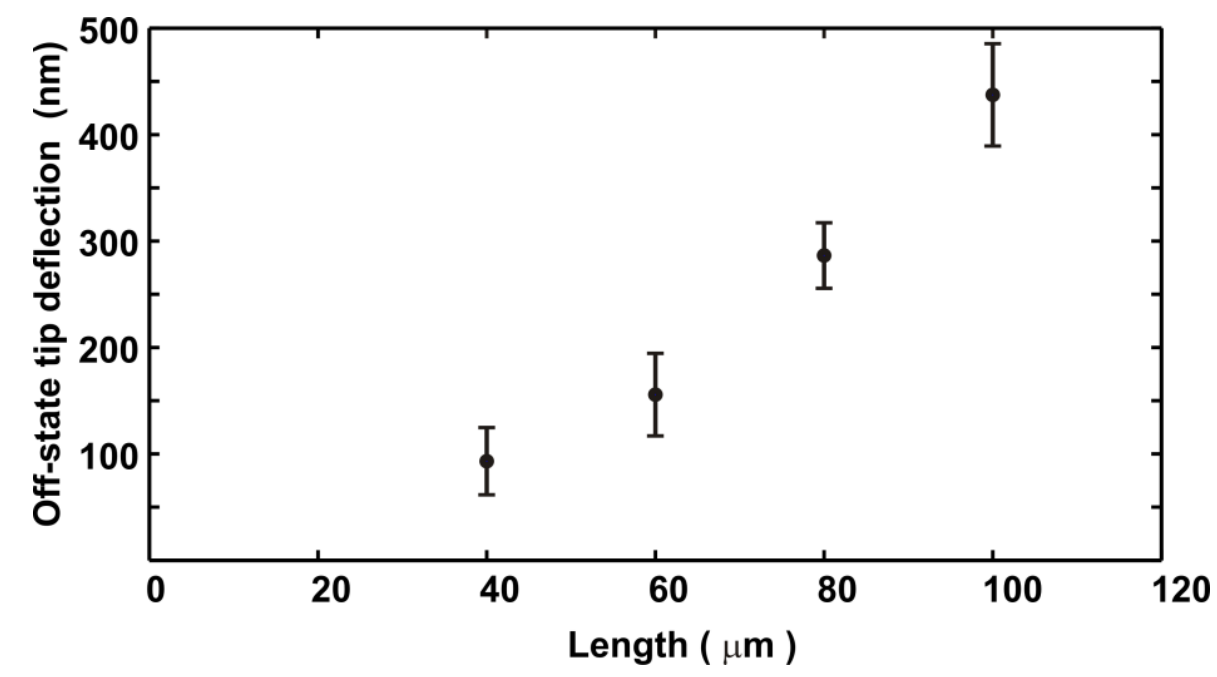

Figure 5.3. Off-state tip deflections of cantilevers of various lengths, measured by WLIM.

Large off-state deflections mean the beams are far away from the MRR compared to the evanescent field decay distance. Considering optical performance this is good, but it also implies that higher (pull-in) voltages are required to achieve appreciable optical modulation. Lower operating voltages can be achieved by increasing the length of the cantilever even though the off-state deflection increases with the length too. However, the length of the beams cannot be increased beyond a critical length as it will result in stiction during the release process or during operation as a result of various adhesion forces [2]. Moreover increasing the length decreases the resonance frequency of the cantilevers, thus reducing their maximum speed of operation.

\subsubsection{Resonance frequency}

Apart from static deflection, micro-cantilevers can be dynamically actuated, e.g. to find their resonance frequencies. The first mode resonance frequency of a microcantilever is an important parameter indicative of its potential time response, thus making it worthwhile to be monitored. Figure 5.4 shows the resonance frequency of a $100 \mu \mathrm{m}$ long cantilever measured with LDV and Figure 5.5 shows the resonance frequencies (left axis) and quality factors (right axis) measured for three cantilever lengths at a constant pressure. As damping at atmospheric pressure makes it difficult to determine resonance frequencies, measurements are carried out at reduced pressure in a vacuum chamber having a proper optical window. 


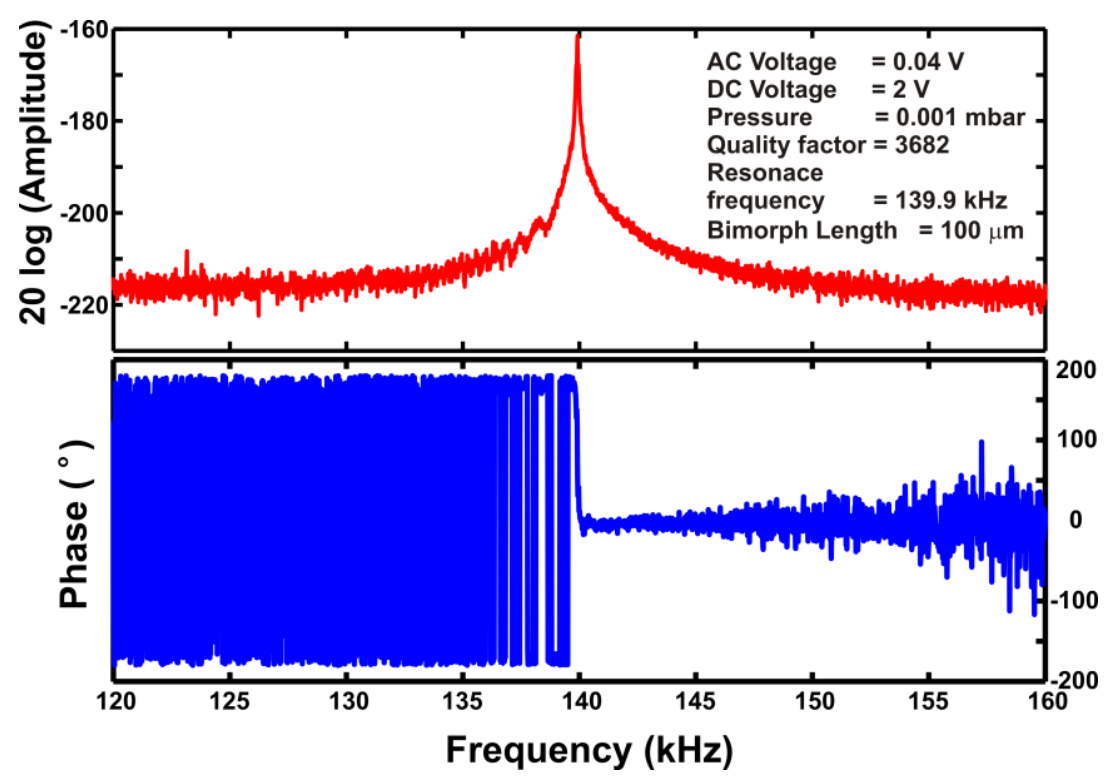

Figure 5.4. Frequency response and corresponding phase shift of a $100 \mu \mathrm{m}$ long cantilever measured at a pressure of 0.001 mbar.

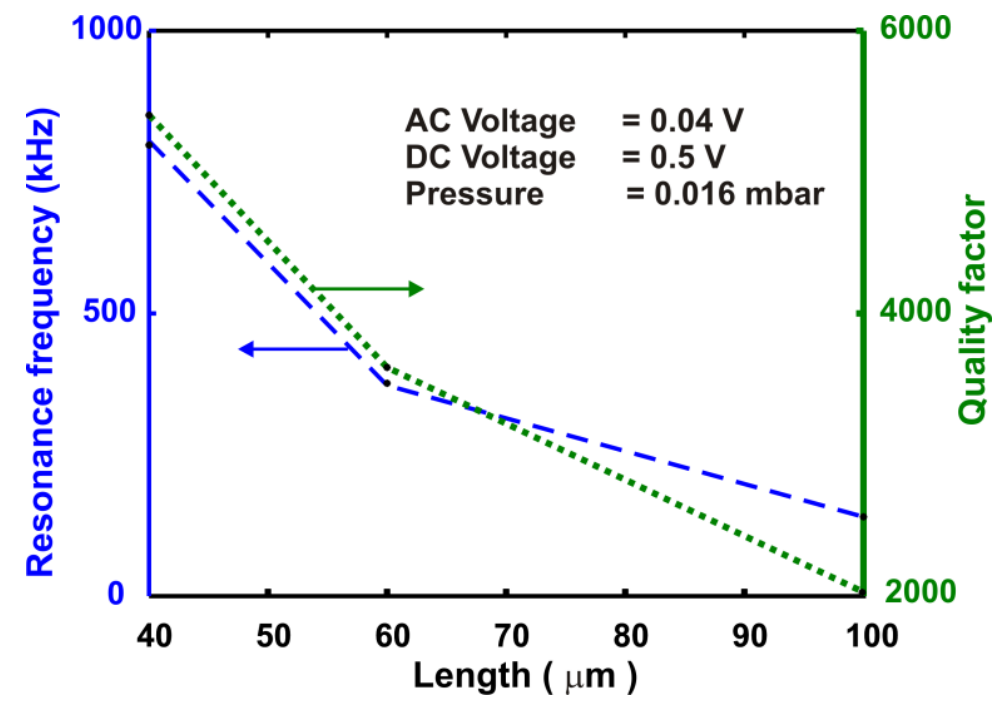

Figure 5.5. Resonance frequency (left axis) and quality factor (right axis) of cantilevers of various lengths measured at a pressure of 0.016 mbar.

To linearly drive an electrostatically actuated cantilever it is customary to use a combination of a relatively large DC and AC voltage. However, the DC component also gives rise to reduction of the effective beam stiffness, an effect known as electrostatic spring softening (see section 3.5.4). So during voltage controlled actuation the effective stiffness of the bimorph is reduced causing a reduction in resonance frequency and quality factor of the cantilevers. Due to these effects it is beneficial to dynamically characterise the cantilevers under varying DC driving conditions.

For these measurements a small AC voltage $\left(V_{\mathrm{AC}}\right)$ of $0.04 \mathrm{~V}$ is applied to excite the bimorphs and the DC voltage is increased to near pull-in values. Reduction in the first 
mode resonance frequency by application of a DC voltage, $f_{1}\left(V_{\mathrm{DC}}\right)$, for bimorphs of three lengths is shown in Figure 5.6.

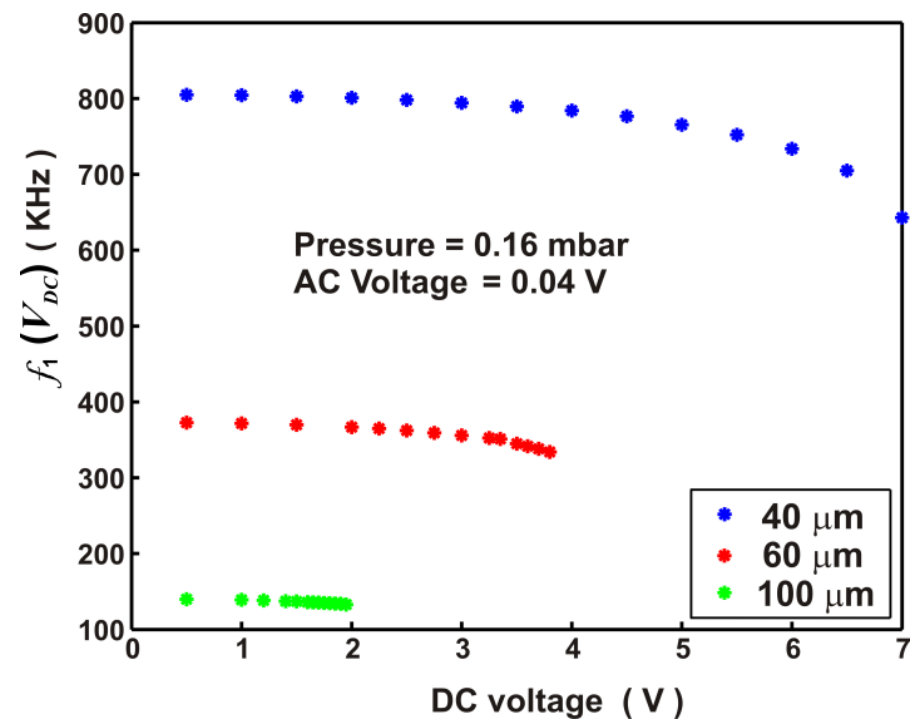

Figure 5.6. Frequency shift of the first mode resonance due to electrostatic spring softening for three bimorph lengths.

Figure 5.7 shows the normalised first mode resonance frequency, i.e. $\left(f_{1}\left(V_{\mathrm{DC}}\right) / f_{1}(0)\right)^{2}$, versus normalised DC voltage squared, i.e. $\left(V_{\mathrm{DC}} / V_{\text {pull-in }}\right)^{2}$ for the same three bimorphs as displayed in Figure 5.6. It is observed that the normalised curves of the three bimorphs largely coincide. This fact can be used beneficially to predict pull-in voltage values from electrostatic spring softening curves like those of Figure 5.6 and as mentioned in section 3.6.2.

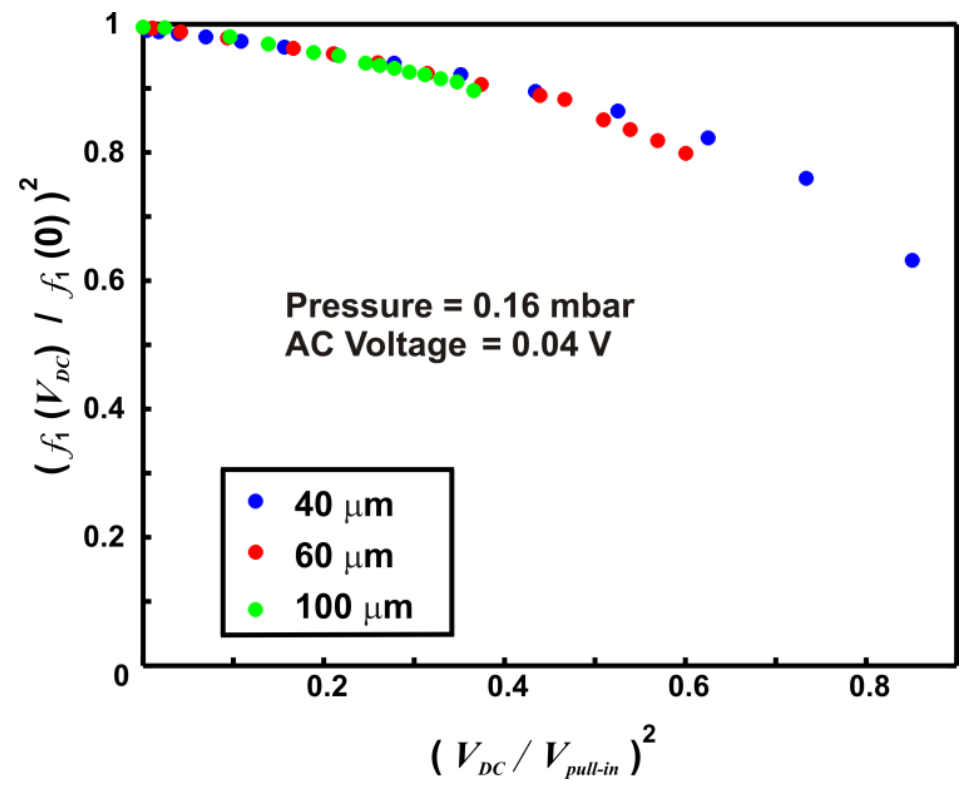

Figure 5.7. Normalised first mode resonance frequency versus normalised actuation voltage for three bimorph lengths. 


\subsubsection{Quality factor}

The quality factor $(Q)$ of a vibrating micro-cantilever is a measure for the amount of energy that can be stored in the cantilever relative to the amount of energy that is lost by damping in each cycle. It depends on various parameters such as geometry, material and the viscosity of the surrounding medium [3]. For large quality factors, it can be determined from the ratio of the resonance frequency $\left(f_{1}\right)$ to resonance peak width $\left(\Delta f_{1}\right)$, the full-width at half maximum (FWHM) of the power curve, and is:

$$
Q=\frac{f_{1}}{\left(\Delta f_{1}\right)_{\text {power }}}
$$

An important step to understand the response of a second order system is to determine whether the system is underdamped, overdamped or critically damped [4]. When $Q<0.5$, the system is said to be over-damped. Over-damped systems are characterised by a lower bandwidth than anticipated from their first mode resonance frequency making it less preferred for switching applications. On the other hand when the $Q>0.5$, the system is called under-damped. Even though the under-damped systems can yield higher bandwidth, the response is accompanied by signal distortion and overshoot, which in turn results in increased settling times. A system is known to be critically damped when $Q=0.5$. Critically damped systems are considered to be fastest among the three, as they comprise the minimum damping needed to avoid any overshoot in their response.

For systems having an air-gap in the sub-micrometer range, the damping is dominated by squeeze film forces at atmospheric pressure [5]. In this device, squeeze film damping arises due to the air trapped between the micro-cantilever and the optical device. In order to understand the effect of damping on the dynamic response of the microcantilever, the pressure dependence of the mechanical quality factor is measured as shown in Figure 5.8. At low pressures the quality factor saturates, where 'low' depends on specifics of the cantilevers. Conversely, for higher pressures the quality factor depends on pressure to the power -0.5 (for a $100 \mu \mathrm{m}$ long beam) and to -1 (for a $40 \mu \mathrm{m}$ long beam). By extrapolating the curves, it is observed that at atmospheric pressures, the $40 \mu \mathrm{m}$ long cantilever has a $Q \sim 1.7$ and the $100 \mu \mathrm{m}$ cantilever has a $Q \sim 1.8$. Hence the cantilevers are observed to be near-critically damped at atmospheric pressure allowing them to be operated nearly as fast as possible for their given resonance frequencies. 
Chapter 5. Micro-cantilever integrated micro-ring resonator: Measurements

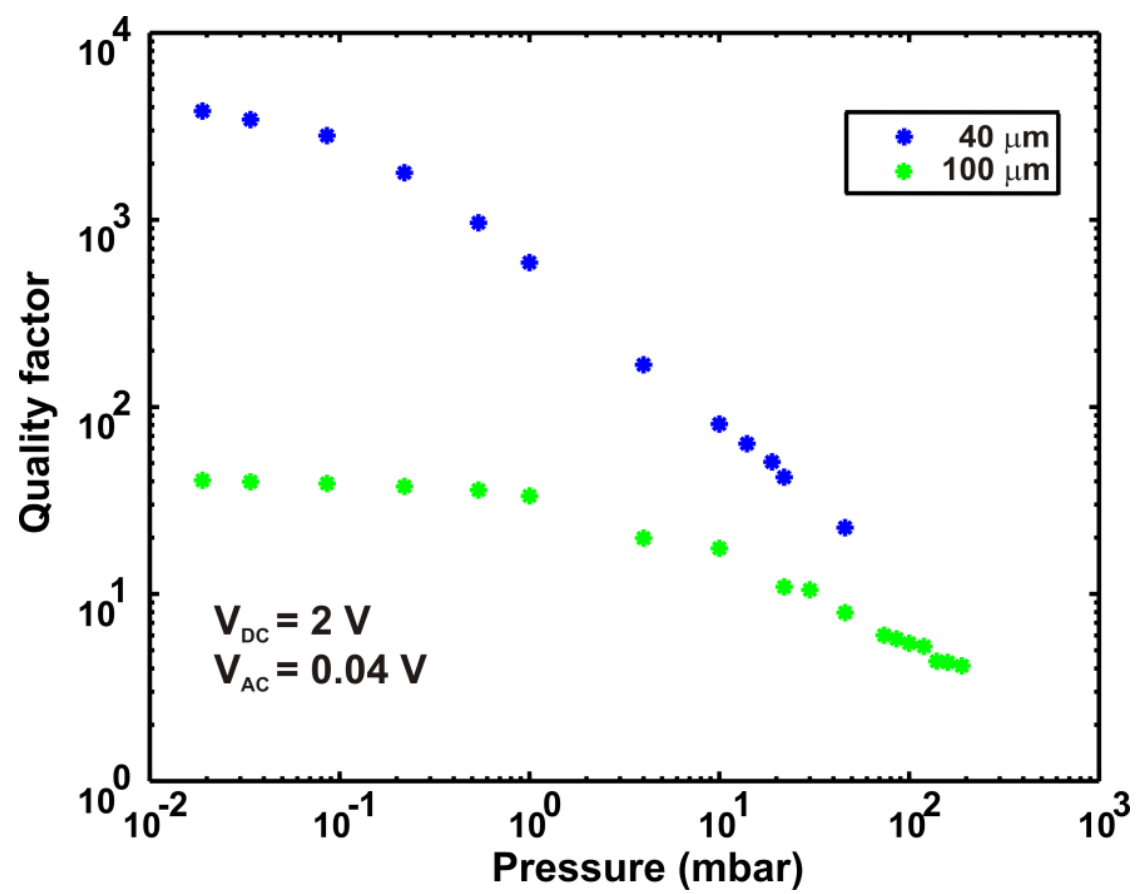

Figure 5.8. Quality factor as a function of pressure for micro-cantilevers of length $40 \mu \mathrm{m}$ and $100 \mu \mathrm{m}$.

At a given pressure ' $P$ ', reduction in air-gap by increasing the applied DC voltage to the micro-cantilever also results in increasing the damping effects. This results in a decrease of the quality factor as shown in Figure 5.9.

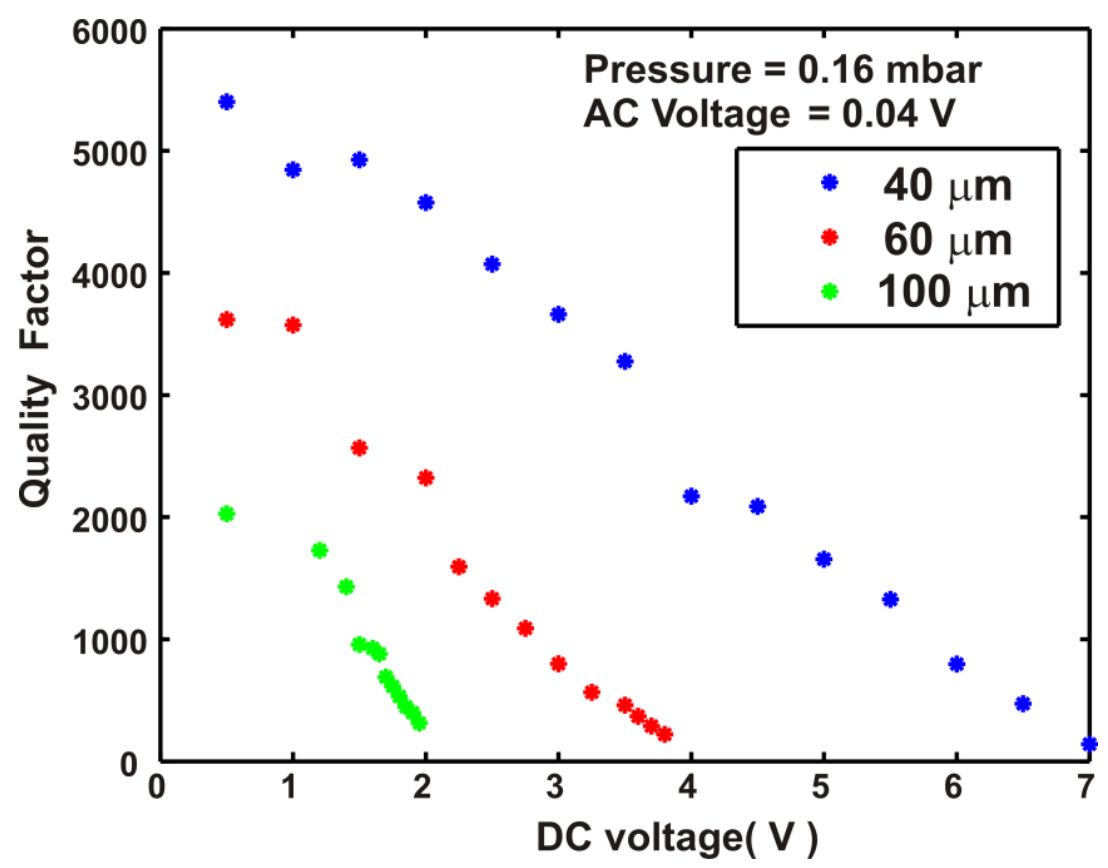

Figure 5.9. Change in quality factor due to ESS effect for three bimorph lengths.

Further, an investigation on the pressure dependence of first mode resonance frequency is carried out. Figure 5.10 demonstrates the change in first mode resonance frequency for a $40 \mu \mathrm{m}$ cantilever with respect to pressure. The resonance frequency is 
normalised with respect to the resonance frequency obtained at the lowest vacuum $\left(f_{\mathrm{vac}}\right.$ at 0.019 mbar). It is observed that an increase in pressure results in an increase in resonance frequency especially in between a pressure region of 1-100 mbar. Similar observation has been also reported in [6] on silicon nitride cantilevers. They have concluded that, at this particular pressure regime (which is in between molecular and viscous region [6]) an increase in pressure has a stiffening effect on the cantilever possibly due to the gas diffusion on the cantilever surface.

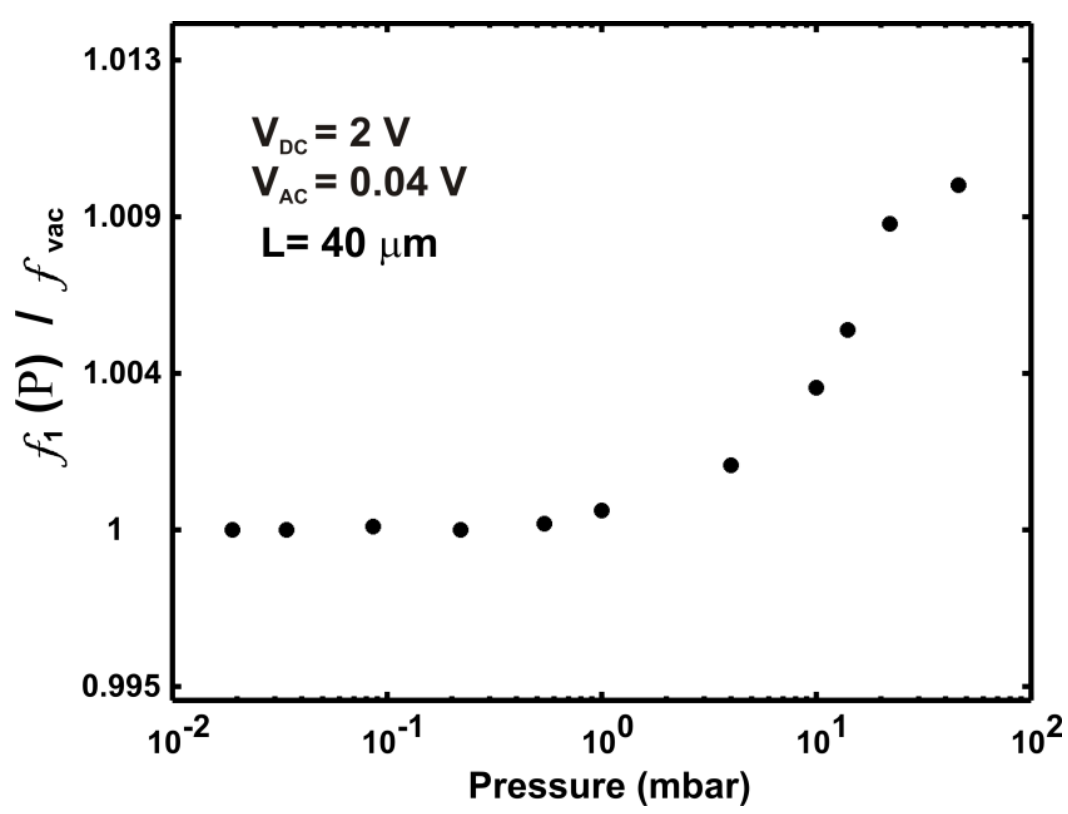

Figure 5.10. Change in first mode resonance frequency with respect to pressure for a $40 \mu \mathrm{m}$ cantilever.

\subsubsection{Response time}

The resonance frequency of the cantilever also gives information about the mechanical bandwidth and rise time. The rise time $\left(t_{95}\right)$-which is the time required for reaching $95 \%$ of its maximum value- is dependent on the mechanical resonance frequency and the quality factor. Figure 5.11 shows a schematic which illustrates the rise time considered. For a second order system $t_{95}$ is given by,

$$
\begin{aligned}
& t_{95}=\frac{6 Q}{\omega_{1}} \quad \text { for } \quad Q>0.5 \\
& t_{95} \approx \frac{10 Q}{\omega_{1}} \quad \text { for } \quad Q=0.5 \\
& t_{95}=\frac{3 Q}{\omega_{1}} \quad \text { for } \quad Q<0.5
\end{aligned}
$$

Where $\omega_{1}=2 \pi f_{1}$. 
Chapter 5. Micro-cantilever integrated micro-ring resonator: Measurements

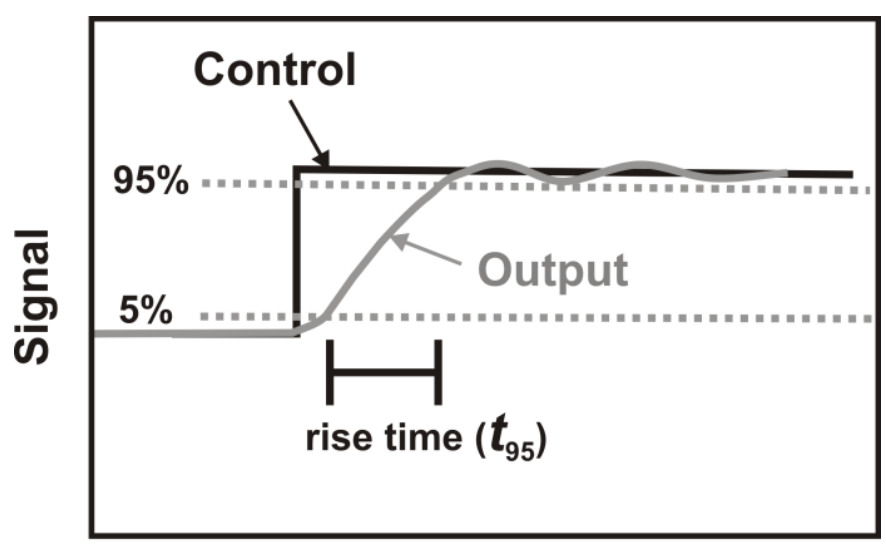

Time (a.u)

Figure 5.11. Schematic showing the rise time and the delay time of the system.

The time response of a $40 \mu \mathrm{m}$ long micro-cantilever is measured by applying a 2 $\mathrm{kHz}$ square wave drive signal $(0-2 \mathrm{~V})$. The measurement is carried out by LDV at atmospheric pressure and the response is shown in Figure 5.12. The measured rise time for this cantilever is $\sim 2.5 \mu \mathrm{s}$, comparable to the value calculated from the quality factor and the resonance frequency as given in Table 5-IV. Additionally the measured response seems to show some slight ringing which would be indicative of a quality factor larger than $1 / 2$ in agreement with the extrapolated value of the quality factor from the pressure dependent measurements.

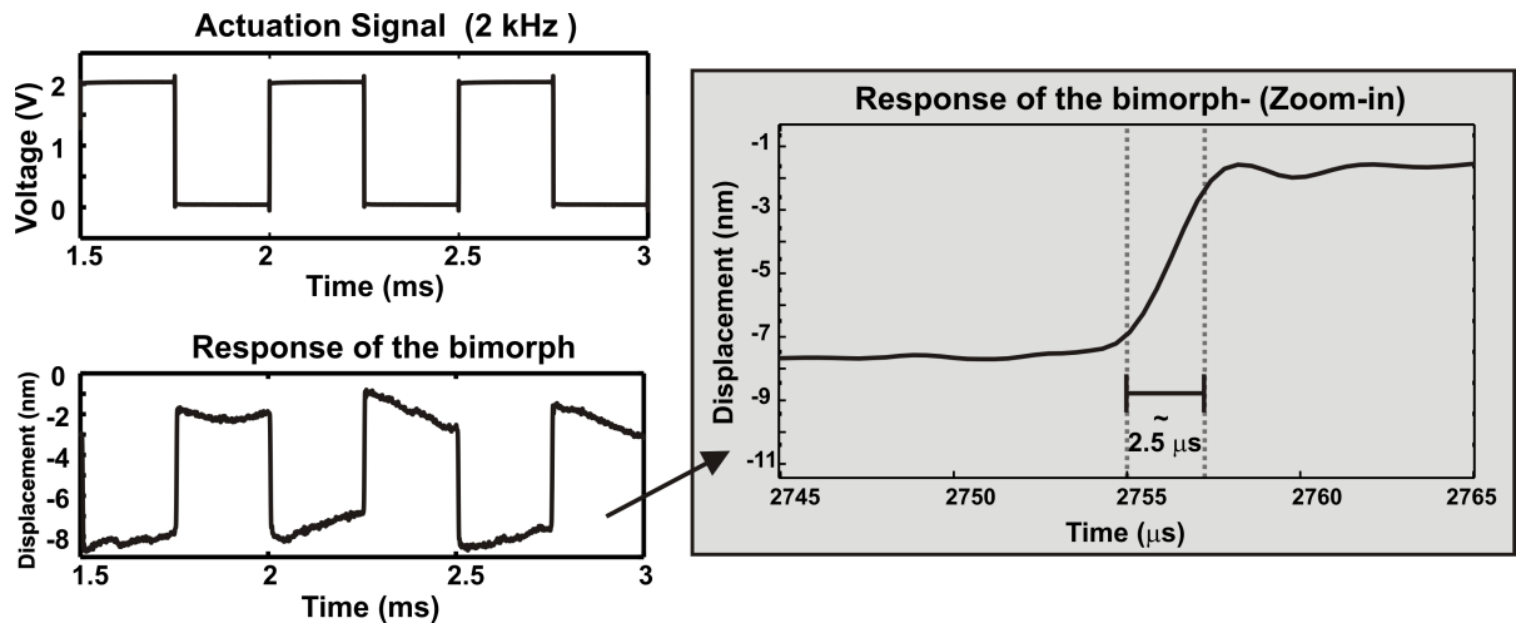

Figure 5.12. Response time of a $40 \mu \mathrm{m}$ long micro-cantilever to a $2 \mathrm{kHz}$ square wave drive signal of 0-2 $V$, measured by $L D V$, at atmospheric pressure.

\subsubsection{Pull-in voltage}

A well-known instability in operating electrostatic MEMS is the 'pull-in' phenomenon as described in detail in section 3.2.3.1 and section 3.6.2. The pull-in voltage $\left(U_{\mathrm{pi}}\right)$ is defined as the voltage beyond which the increasing restoring spring force can no longer keep up with the even faster increasing electrostatic attractive force [16] and at this 
voltage the cantilever is pulled to the counter electrode. Pull-in can be the cause of the failure, e.g. when stiction occurs after pull-in. Hence it is important to determine the pullin voltage and to estimate the stable range of operation of the cantilever. The pull-in voltage strongly depends on the effective gap between the two electrodes. The effective gap is a combination of the air-gap and the effective dielectric gaps where the latter can be found from dividing the thickness of each dielectric layer by its relative permittivity $\left(\varepsilon_{\mathrm{r}}\right)$. Since the $220 \mathrm{~nm}$ silicon device layer consist of only small area below the cantilever (see Figure 5.2B), this layer has not been considered for the calculation Figure 5.13 shows the various parameters considered for the effective gap calculation for a $60 \mu \mathrm{m}$ long cantilever, which is given by,

$$
g_{\text {eff }}=\frac{2000}{3.9}+\frac{40}{7.5}+196+102+\frac{1000}{7.5} \approx 950 \mathrm{~nm}
$$

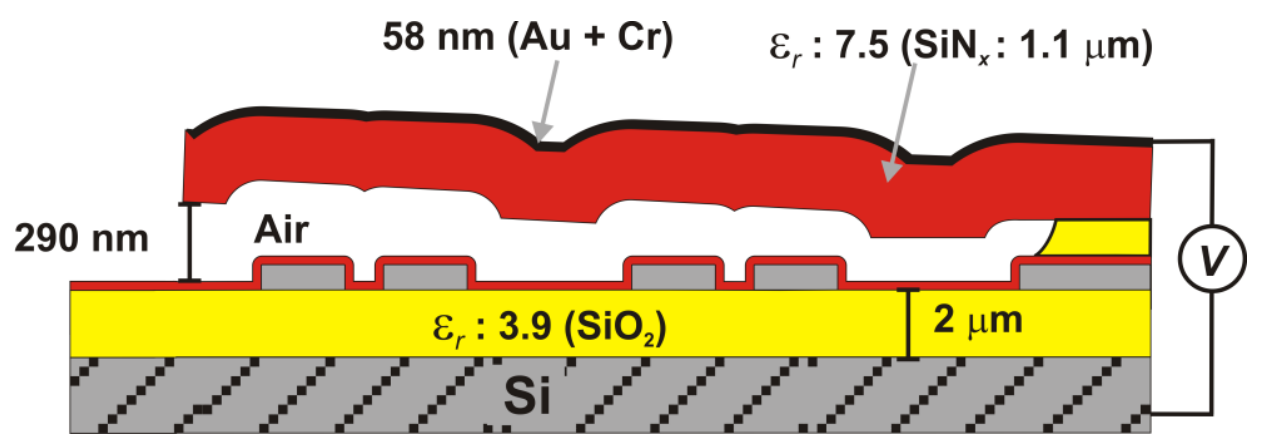

Figure 5.13. Various parameters considered in the effective gap calculation for a $60 \mu \mathrm{m}$ long cantilever.

Next the pull-in voltage is predicted for the integrated device using the Galerkin based static model described in section 3.2.3.1. Parameters used for the gold layer are listed in Table 5-I.

Table 5-I. Material parameters [7] of the gold (Au) layer.

\begin{tabular}{|l|c|}
\hline \multicolumn{1}{|c|}{ Variables } & Values \\
\hline Young's Modulus, $E_{\mathrm{Au}}(\mathrm{GPa})$ & 78 \\
\hline Relative dielectric constant, $\varepsilon_{\mathrm{Au}}$ & 3.9 \\
Density, $\rho_{\mathrm{Au}}\left(\mathrm{Kg} / \mathrm{m}^{3}\right)$ & 19320 \\
\hline Thermal expansion coefficient, $\alpha_{\mathrm{Au}}\left(\mathrm{K}^{-1}\right)$ & $14.2 \times 10^{-6}$ \\
\hline
\end{tabular}

In order to include the effect of undercut of the bond pads introduced by the sacrificial layer etching step, the deviation in effective length $(\Delta L)$ and deviation in effective flexural rigidity $(\Delta E I)$ are calculated from the frequency response measurements (see section 3.6.1). Table 5 -II shows the values considered for this calculation which gives $\Delta L=2.37 \mu \mathrm{m}$ and $\Delta E I=-0.1076$. 
Table 5-II. Parameters considered for effective $\Delta L$ and $\Delta E I$ calculation.

\begin{tabular}{|c|c|c|c|c|}
\hline $\begin{array}{c}\text { Length } \\
(\boldsymbol{\mu m})\end{array}$ & $\begin{array}{c}f_{\text {measured }} \\
\mathbf{( k H z )}\end{array}$ & $\begin{array}{c}f_{\text {predicted }} \\
\mathbf{( k H z )}\end{array}$ & $\begin{array}{c}\Delta f \\
\mathbf{( k H z )}\end{array}$ & $\frac{\Delta f}{f_{\text {predicted }}}$ \\
40 & 804.93 & 860.9 & 55.97 & 0.0650 \\
60 & 372.868 & 382.6 & 9.73 & 0.0254 \\
\hline
\end{tabular}

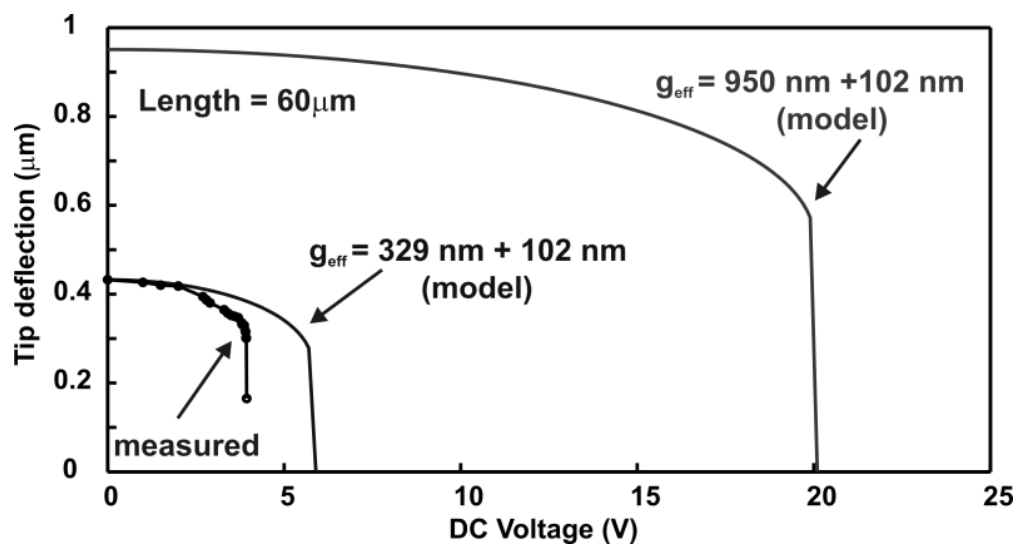

Figure 5.14. Comparison between the pull-in curves predicted by the static Galerkin model and measured values, for a $60 \mu \mathrm{m}$ long cantilever. Model predictions are carried out for case $A$ (red) and B (blue);see text.

Figure 5.14 shows a comparison between the model predictions and the actual measurements on pull-in voltage. The model predictions are considered for two cases (A) the silicon device layer is considered as the bottom electrode and (B) the silicon handle wafer is considered as the bottom electrode. Obviously the effective gap strongly differs for both the cases which will yield different pull-in voltages. For case A, $g_{\text {eff }}=329 \mathrm{~nm}+$ $196 \mathrm{~nm}$ which are due to the contributions from air gap, off-state deflection of the cantilever, effective gap from the $\mathrm{SiN}_{x}$ cantilever and protective nitride layers. On the other hand, case B has a $g_{\text {eff }}=950 \mathrm{~nm}$, as given by equation( 0.3$)$.

Table 5-III. Comparison between measured and predicted pull-in voltages

\begin{tabular}{|c|c|c|c|c|c|}
\hline $\begin{array}{c}\text { Length } \\
(\mu \mathrm{m})\end{array}$ & $\begin{array}{c}\text { Off-state tip } \\
\text { deflection } \\
(\mathbf{O S D} \text { in } \\
\mathbf{n m})\end{array}$ & $\begin{array}{c}\text { Measured } \\
U_{\mathrm{pi}}(\mathbf{V})\end{array}$ & $\begin{array}{c}U_{\mathrm{pi}}(\mathbf{V}) \\
\text { predicted } \\
\text { from ESS } \\
\text { measurements }\end{array}$ & $\begin{array}{c}U_{\mathrm{pi}}(\mathbf{V}) \text { predicted } \\
\text { by model with } \\
g_{\text {eff }}=329+\text { OSD }\end{array}$ & $\begin{array}{c}U_{\mathrm{pi}}(\mathbf{V}) \text { predicted } \\
\text { by model with } \\
g_{\text {eff }}=950+\text { OSD }\end{array}$ \\
40 & 93 & 9.2 & 8.3 & 12.2 & 42.9 \\
60 & 103 & 3.94 & 5.3 & 5.9 & 20.1 \\
100 & 437 & 2.2 & 3.5 & 3.9 & 10.1 \\
\hline
\end{tabular}


An overview of measured and the predicted pull-in voltages is given in Table 5-III. A prediction in $U_{\mathrm{pi}}$ from the extrapolation of the ESS measurements on resonance frequency, which was presented in Figure 5.6 is given as well. Detailed analysis by Tas et.al. [2] show that for a straight, initially stress-free cantilever, the pull-in instability point is not 0.33 , but rather 0.44 of the effective gap, $\left(g_{\text {eff }}\right)$. Although the cantilever in this study is not straight and has built-in stress, if we assume that instability point is close to $0.44 g_{\text {eff }}$, the pull-in instability should not have been observed in this device. However, the device clearly shows pull-in behaviour. It is not understood why this happens and a more detailed analysis is required.

Static measurements of cantilever displacements were carried out in small voltage steps $(0.01 \mathrm{~V})$ to conform whether the observed down-state positions of the cantilever on the substrate were indeed the result of pull-in instability or were the result of stable displacements, presumably followed by stiction of the cantilever to the substrate. Figure 5.15 shows static pull-in measurements by WLIM for a $60 \mu \mathrm{m}$ long cantilever, which has a pull-in instability point at a DC voltage of $3.94 \mathrm{~V}$. The device shows a sudden instability from $3.93 \mathrm{~V}$ to $3.94 \mathrm{~V}$ and gets permanently stuck to the surface, indicating clear pull-in behaviour. The measurement is performed on a test cantilever, on the same chip, but not integrated with a MRR. Further Figure 5.16 shows the shape of a $40 \mu \mathrm{m}$ long cantilever at its off-state $(0 \mathrm{~V})$ and at pull-in $(9.2 \mathrm{~V})$.

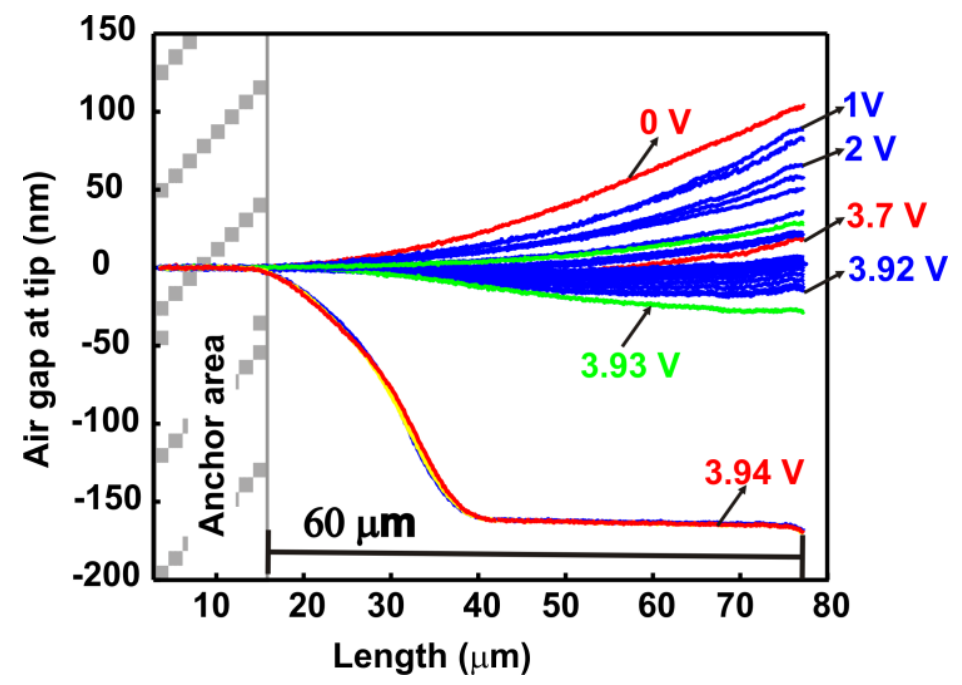

Figure 5.15. Static tip deflection as a function of applied actuation voltage for a $60 \mu \mathrm{m}$ long microcantilever (test device). 
Chapter 5. Micro-cantilever integrated micro-ring resonator: Measurements

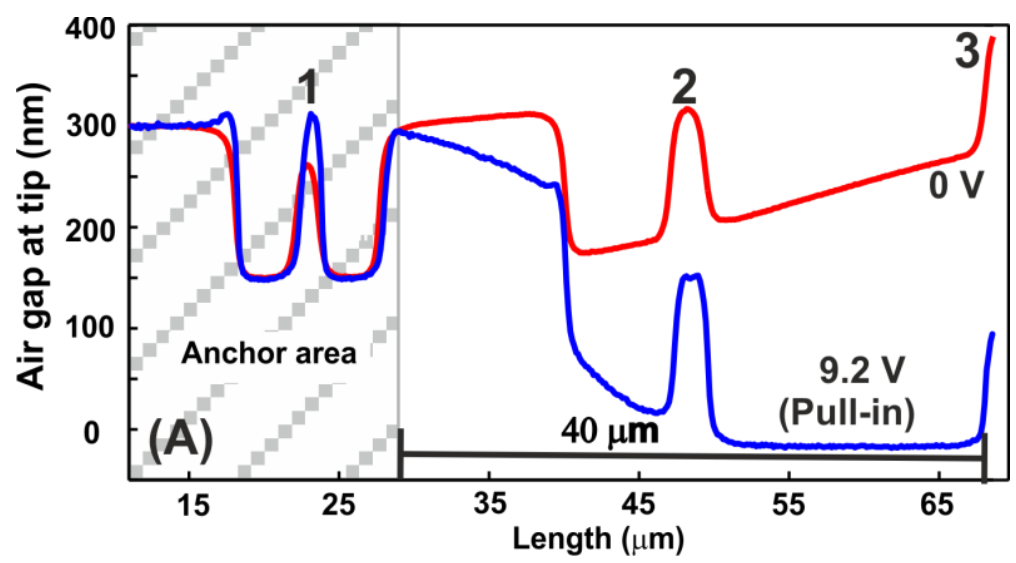

Figure 5.16. Shape of a $40 \mu \mathrm{m}$ long cantilever integrated with an $M R R$ at the off-state $(O \mathrm{~V})$ and at pull-in $(9.2 \mathrm{~V})$.

\subsubsection{Tapered gap}

After sacrificial layer etching (SLE), the thickness of the remaining protective $\mathrm{SiN}_{x}$ (PL) in the freely accessible areas is approximately $14 \mathrm{~nm}(40-52 \times 0.5 ; 40 \mathrm{~nm}$ being the thickness of the $\mathrm{SiN}_{x}(\mathrm{PL}), 52$ min being the sacrificial layer etch time and $0.5 \mathrm{~nm} / \mathrm{min}$ being the etch rate of $\mathrm{SiN}_{x}$ in $\mathrm{BHF}$ ). However, the PL underneath the cantilever will have a tapered profile as the sacrificial layer (SL) is covering the PL during part of the SLE time [8]. This effect is shown in Figure 5.17. Below the centre of the cantilever the PL thickness variation can be as high as $16 \mathrm{~nm}$.

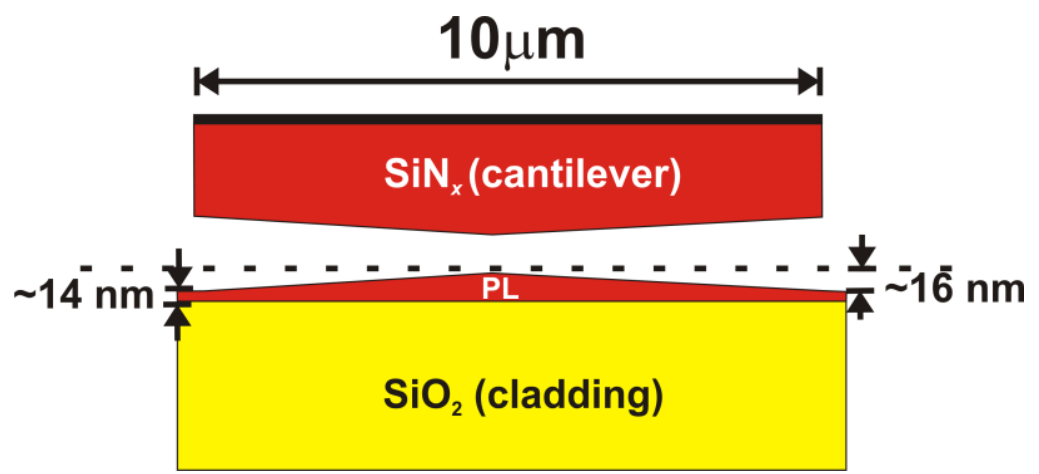

Figure 5.17. Variation in the PL and cantilever layer thickness below the cantilever, after the SLE in BHF. 

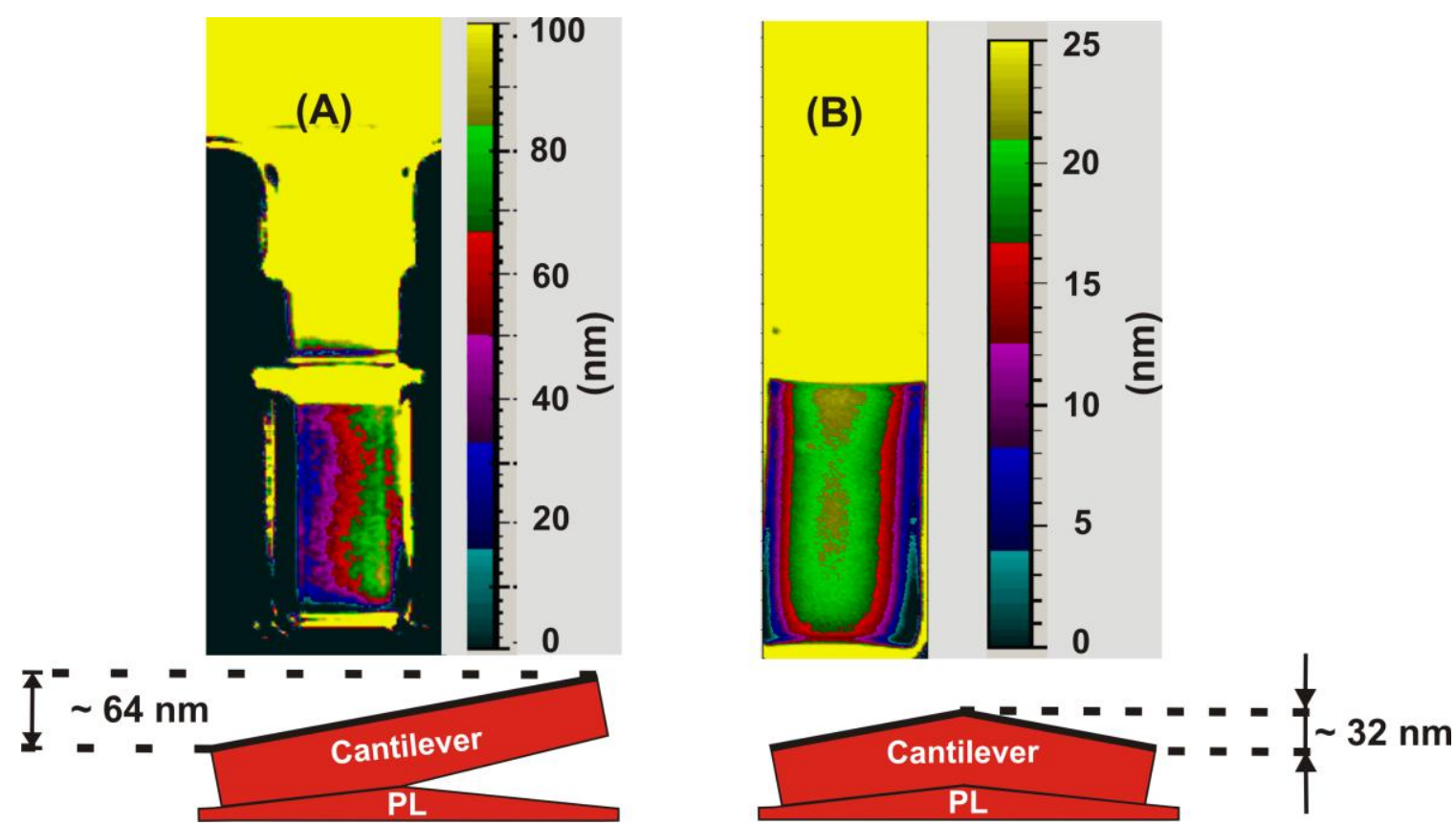

Figure 5.18. WLI images showing two different shapes of the beams after pull-in, (A) balf sticking: beam tip is tilted, (B) full sticking: beam tip is deformed.

In addition to the PL underneath the cantilever, the bottom side of the cantilever itself will have a tapered profile as well. Due to this, at pull-in, the cantilever can either stick completely or partially (half) at the substrate. Half sticking will force the cantilever to tilt either to the left or the right (Figure 5.18A), whereas full sticking will deform the beam as shown in Figure 5.18B. One way to prevent stiction is by introducing holes into the cantilever as shown in Figure 5.19. The extra holes will have at least two positive effects. First, it will reduce the time needed for SLE and so relax the choice for the thickness of the SL and PL. Second, it will introduce a saw tooth etch profile under the cantilever, which might prevent intimate contact between the approaching surfaces. 
Chapter 5. Micro-cantilever integrated micro-ring resonator: Measurements

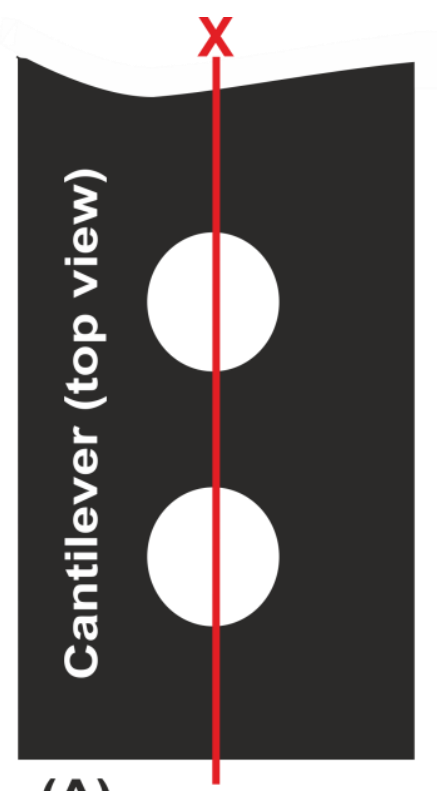

(A)

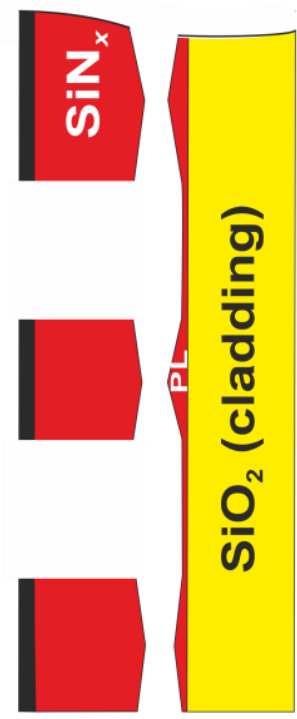

(B)

Figure 5.19. (A) Top view and (B) cross-sectional view in X-direction (defined in Figure. 4.1) of (part of) a cantilever having etch holes which will create a saw toothed etch profile underneath.

\subsection{Optical characterisation}

\subsubsection{Measurement set up}

Racetrack ring resonator with integrated micro-cantilevers, were optically characterized to assess their performance. Infrared light from a tunable laser (input power of $1 \mathrm{~mW}$ ) was coupled through integrated grating couplers [9], to the input waveguide, using a polarization maintaining fiber (PMF). A polarization controller was used to control the polarisation state of the light between the laser and the PMF. The transmitted light from the 'through' and the 'drop' port waveguides was recollected by second and third gratings, and guided to a photodetector (Agilent 8164B and 81634B) through a single mode fiber (SMF).

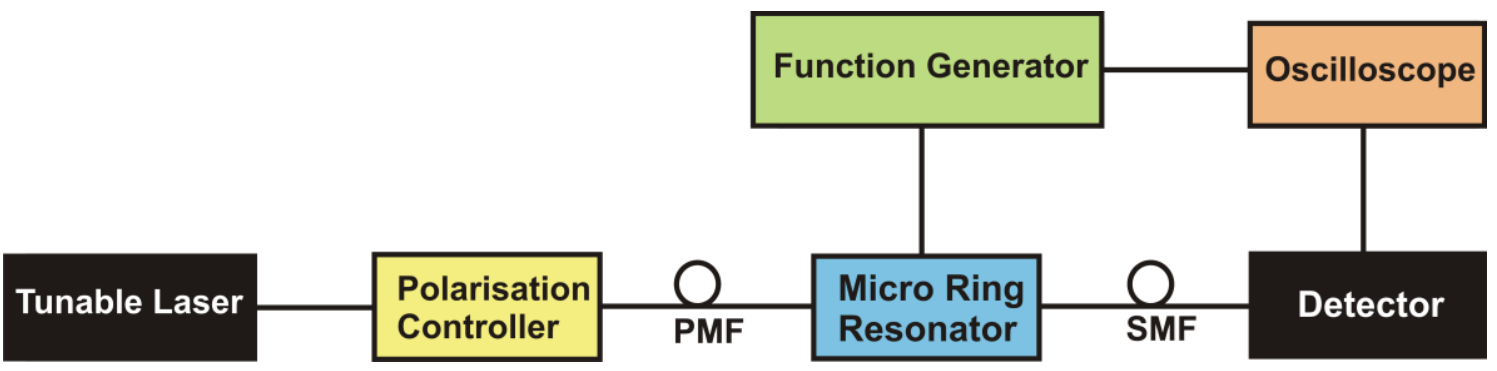

Figure 5.20. Schematic representation of the optical modulation measurement set up.

Figure 5.20 shows the schematic of the measurement set up used for optical modulation. A function generator is used to apply the modulation voltage to the integrated device using electrical probes. In order to avoid electrical insulation by native 
oxide formation on the silicon handle wafer, the wafer is silver glued to a copper plate, onto which the lower electrical contact is made. Figure 5.21 shows the SEM image of one of the grating couplers which is at the end of an access waveguide.

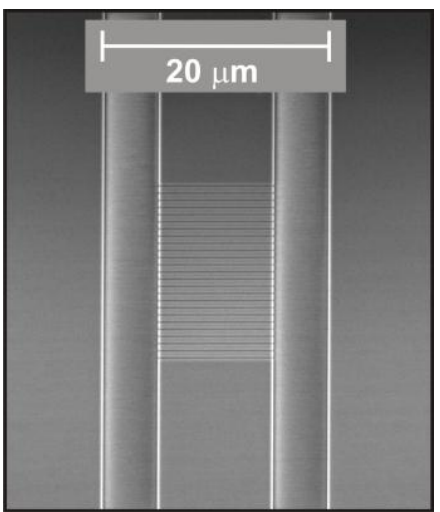

Figure 5.21. An SEM image of one of the grating couplers at the end of an access waveguide.

Figure 5.22A shows photograph of the experimental set up in which the optical chip is connected with electrical probes and fibre couplers. The optical chip is placed on a vacuum chuck in order to avoid movement. After coupling the cleaved fiber ends into and out of the grating couplers, the electrical probe (for upper electrode) is carefully connected on the cantilever bondpad. The probe for the lower electrode is connected to the copper plate. Figure 5.22B shows a more detailed image of the probe sitting on the cantilever bondpad, with the cleaved fiber ends on either side of the probe.
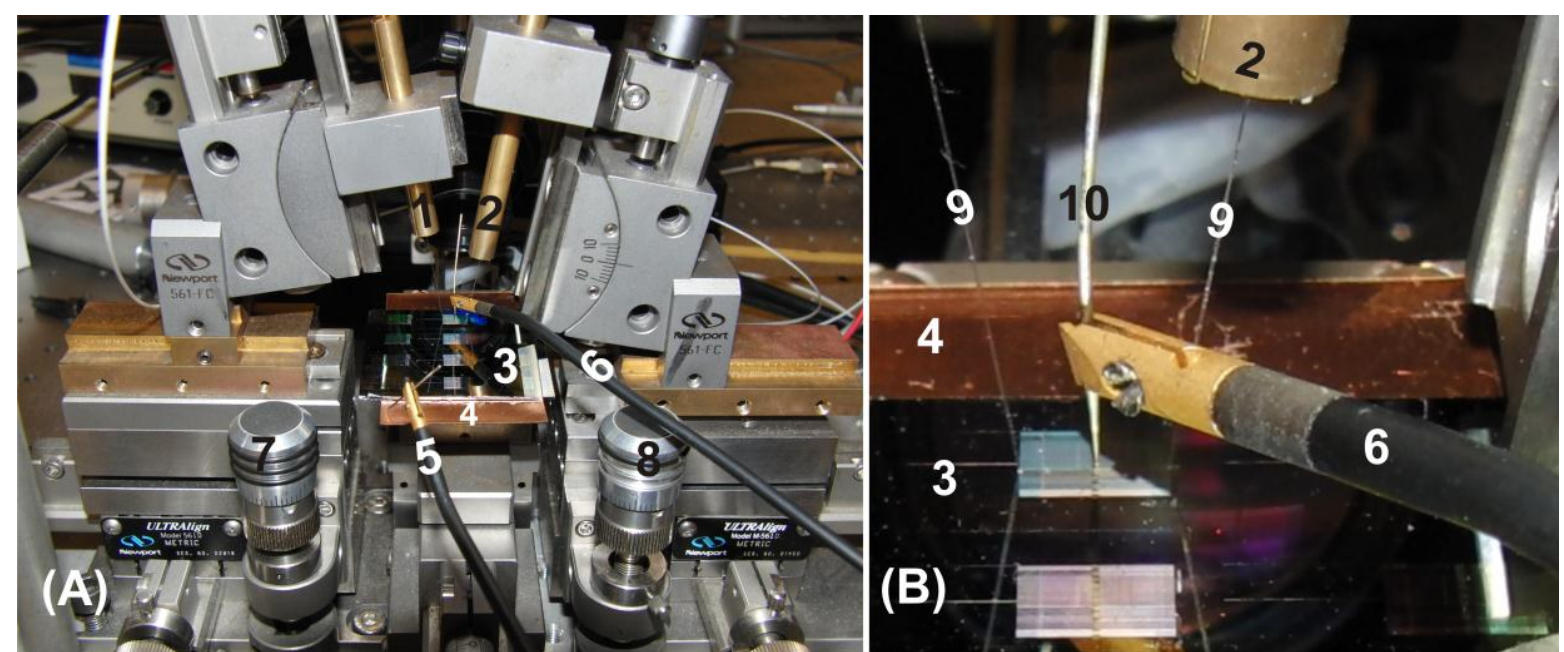

Figure 5.22. Photographs of the experimental set up. [1.Fibre In, 2. Fibre Out, 3.Integrated chip, 4. Copper plate, 5. Electrical probe to the ground, 6, 10. Electrical probe to the cantilever, 7, 8. Actuators for positioning the fibres, 9. Cleaved fibre tips]. 
Chapter 5. Micro-cantilever integrated micro-ring resonator: Measurements

\subsubsection{Optical losses}

A typical response measured in the 'through' and 'drop' ports (of the device shown in Figure 5.1), is shown in Figure 5.231. The racetrack resonator has a measured free spectral range of $6.6 \mathrm{~nm}(=1558.1-1551.5)$. All the optical measurements discussed in this chapter are performed with a resolution of $5 \mathrm{pm}$.

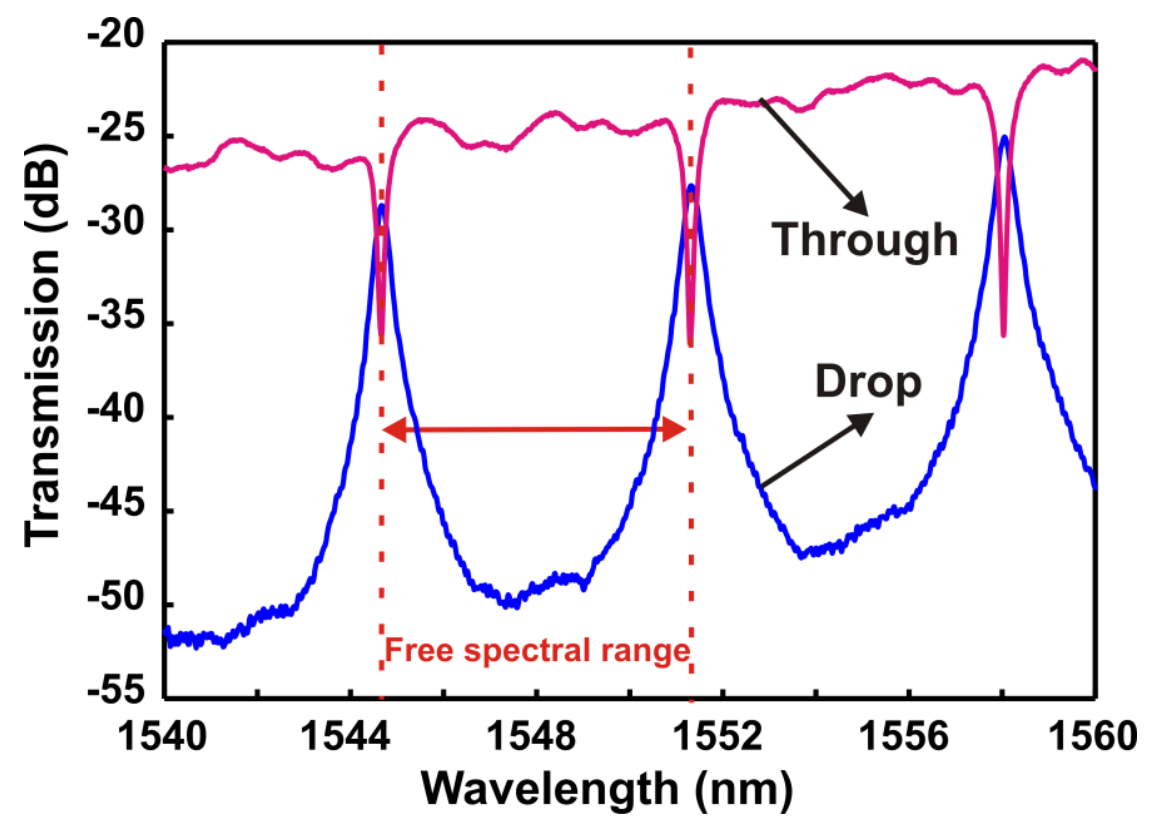

Figure 5.23. Through and drop response of the fabricated racetrack ring resonator after cantilever integration.

The optical loss in the device is another parameter which has to be analyzed. For this, the transmission in a straight waveguide (length $=6 \mathrm{~mm}$ and width $=600 \mathrm{~nm}$ ) and a ring resonator on the same chip, before and after the cantilever integration are measured (Figure 5.24). It is observed that after post processing the transmission in the targeted wavelength range of 1530-1565 nm changed. This could be a red shift of the transmission spectrum of the grating couplers [9] due to the added high-index PL (Figure 5.24) and given the fact that fibre-to-grating coupling was not adjusted for the post-processed devices. In any case the $20-25 \mathrm{~dB}$ losses observed in Figure $5.23 \mathrm{~A}$ are not inherent to the ring resonator, but are the overall loss by the system, which includes and is dominated by coupling and propagation losses. The loss introduced by the ring-resonator is negligibly small $(<3 \mathrm{~dB}[10])$.

1 This measurement has been carried out on an integrated device where the cantilever is stuck on the MRR. Hence the quality factor of the device is less than the quality factor of the measurement which is shown in Figure.5.25. A more detailed discussion regarding the reduction in quality factor at pull-in is given in section 5.3.3. 

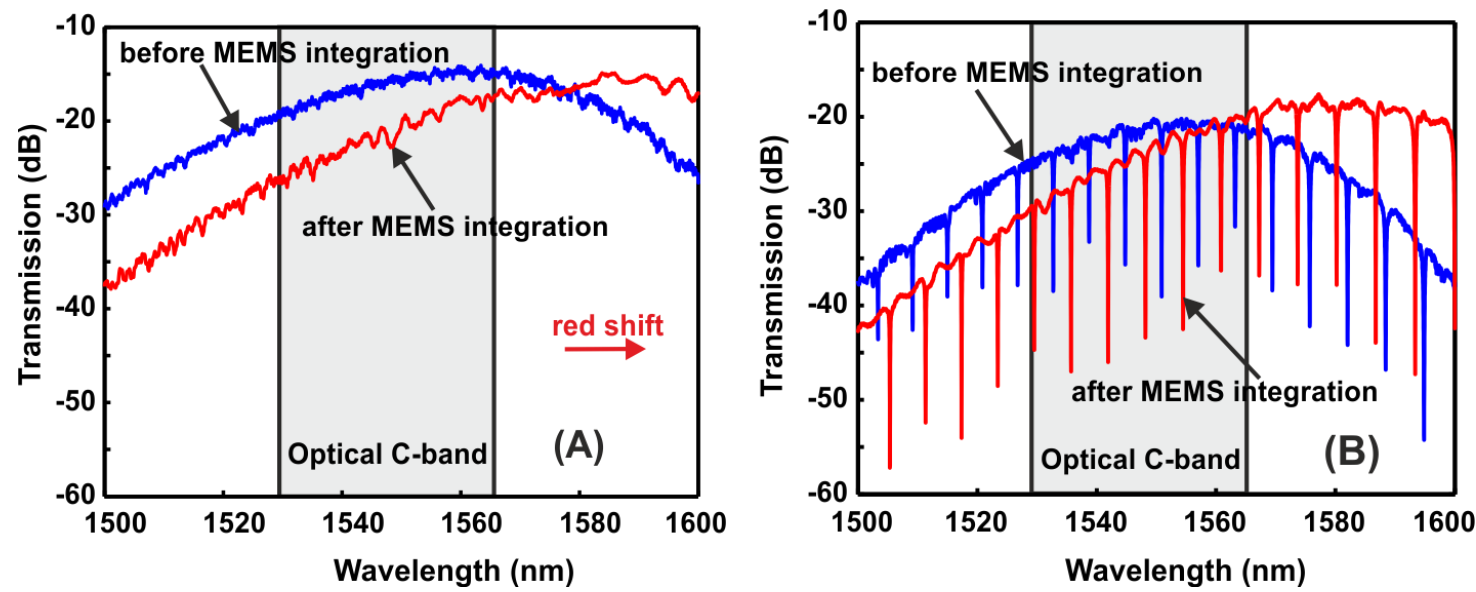

Figure 5.24. Transmission measured in (A) straight waveguide and (B) ring resonator, before and after the cantilever integration.

\subsubsection{Wavelength tuning}

Results of the tuning experiments on a micro-ring resonator integrated with cantilever of $40 \mu \mathrm{m}$ length are shown in Figure 5.25-Figure 5.27. The spectrum measured in through port shows a $20 \mathrm{~dB}$ drop of transmission at a resonance wavelength of $1559.57 \mathrm{~nm}$ (Figure 5.25). Resonance wavelength tuning of $122 \mathrm{pm}$ and a modulation depth of $18 \mathrm{~dB}$ are measured for a voltage of up to $9 \mathrm{~V}$. In the stable range of operation of the cantilever, the quality factor of the resonance remains unchanged within $2 \mathrm{~dB}$ (Figure 5.25), indicating that the cantilever does not introduce any substantial losses to the resonator. Figure 5.26 shows the measured through port wavelength shift as a function of the applied voltage. The effect is highly nonlinear showing an increased shift in wavelength when the cantilever is more close to the optical evanescent field. By having a stable range in cantilever operation in the close proximity of the ring resonator, much higher wavelength shift can be achieved. More details regarding the measurements will be discussed in section 5.4.

At pull-in (9.2 V, Figure 5.27), the cantilever touches the waveguide and a moderate reduction in the quality factor of the ring resonator (from $\sim 8000$ to $\sim 5000$ ) as well as a considerable change $(5 \mathrm{~nm})$ in the resonance wavelength is observed. However the shift may be larger considering the fact that the response is periodic in wavelength. The latter observation might give this device the ability to switch optical signals in an effective way. 
Chapter 5. Micro-cantilever integrated micro-ring resonator: Measurements

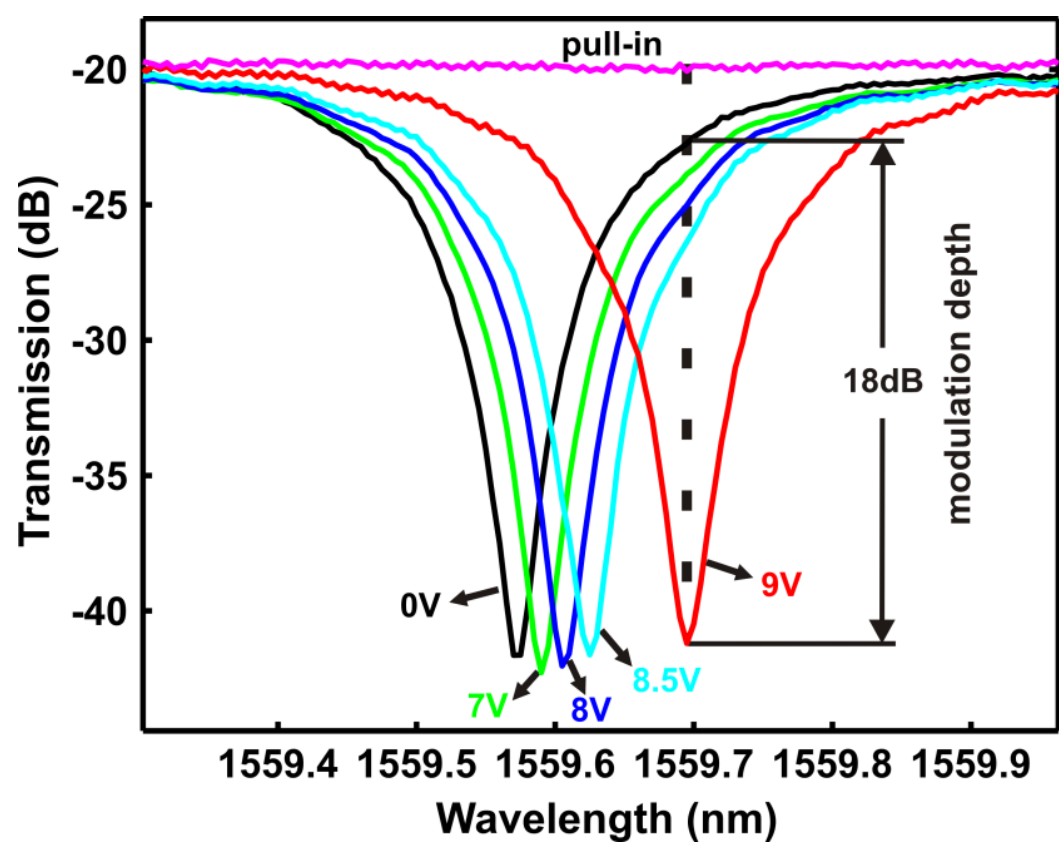

Figure 5.25. Static wavelength tuning up to 122 pm by application of a voltage of $9 \mathrm{~V}$.

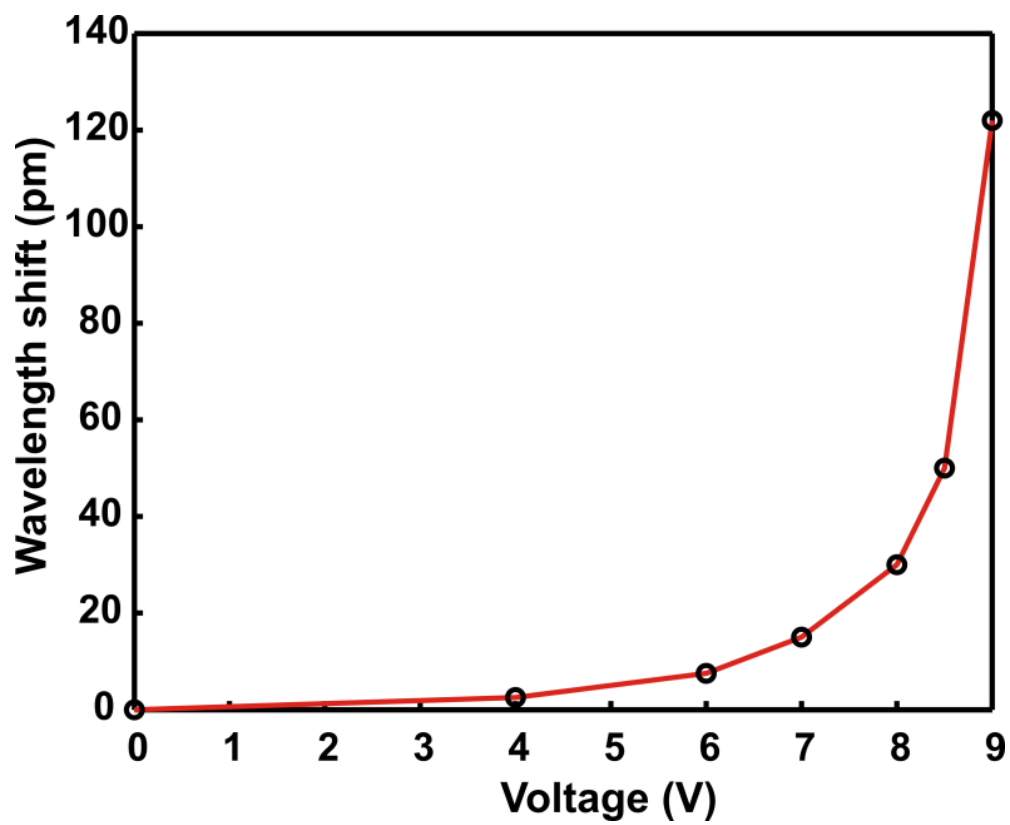

Figure 5.26. Measured wavelength shift as a function of the applied voltage. 


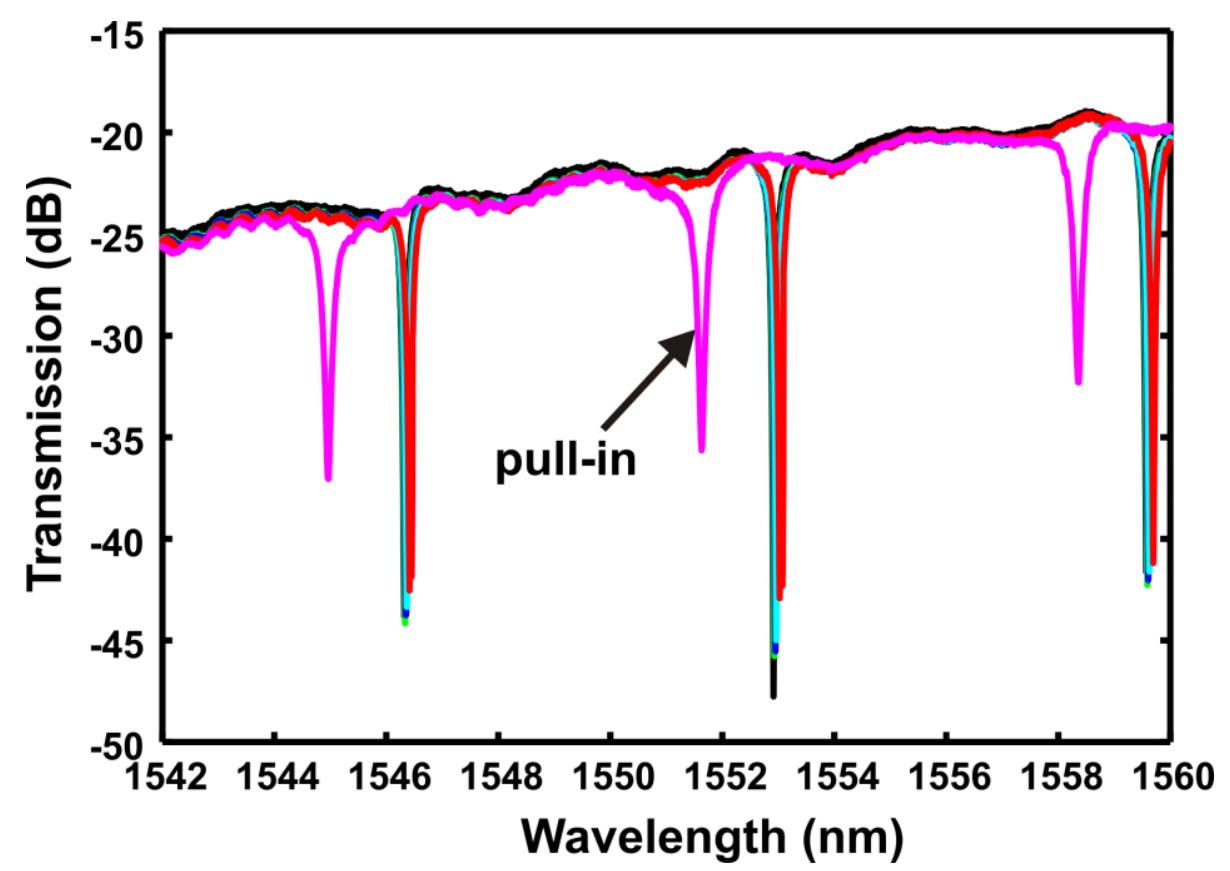

Figure 5.27. Resonance wavelength shifting at the pull-in of a $40 \mu \mathrm{m}$ long micro-cantilever.

\subsubsection{Dynamic switching}

Dynamic switching measurements are performed at a fixed wavelength, tuned to the optical resonance of the micro-ring resonator. On application of a square wave drive signal of $0-4 \mathrm{~V}^{2}$ at $1 \mathrm{kHz}$ and at $30 \mathrm{kHz}$, for a $100 \mu \mathrm{m}$ long cantilever, optical modulation is observed. A $95 \%$ signal rise time of $\sim 16 \mu$ s and fall time of $\sim 5 \mu$ s are found from the measurements displayed in Figure 5.28. The reason for the unequal rise and fall times might be due to the spring softening of the cantilever due to a voltage controlled actuation (e.g. see Figure 5.12 for the case of a $40 \mu \mathrm{m}$ long cantilever).

2 The measured pull-in voltage of a $100 \mu \mathrm{m}$ cantilever is $2.2 \mathrm{~V}$ (see Table 5-III). However the pull-in voltage varies from cantilever to cantilever of same length on the same wafer. One reason for this is due to a variation in off-state deflection of the cantilever. The $100 \mu \mathrm{m}$ long cantilever used in the dynamic switching measurement (Figure 5.28), shows a stable range of operation even at 4V. Certainly the cantilever is not pulled in this measurement, as a pulled cantilever will be permanently stuck to the optical device and will not show any optical modulation. 
Chapter 5. Micro-cantilever integrated micro-ring resonator: Measurements
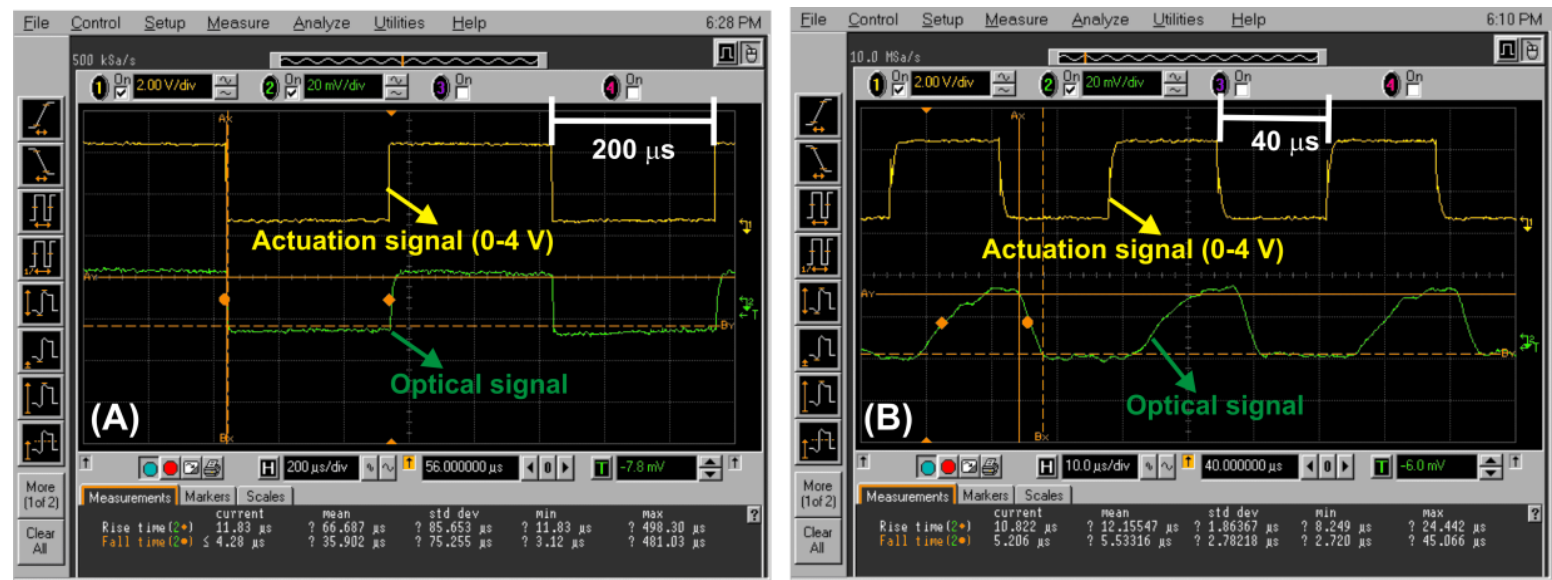

Figure 5.28. Optical modulation measured for a $0-4 \mathrm{~V}$ square wave (A) at $1 \mathrm{kHz}$ and (B) at 30 kH\%:

\subsection{Discussion}

This section gives a detailed discussion regarding the mechano-optical performance of the integrated device. From Figure 5.25 it is evident that mechano-optical modulation enables a significant shift in the resonance frequency without introducing optical losses, i.e. only a voltage change from 8.5 to $9 \mathrm{~V}$ is enough for realizing a wavelength shift of $70 \mathrm{pm}$. The tunability range strongly depends on the distance between the cantilever and the ring resonator. A higher tuning range can be achieved by increasing the stable range of operation of the cantilever. Obviously the tuning range can also be increased by increasing the interaction length between the ring and the cantilever. At present the cantilever covers only one by third of the ring which includes the coupling sections between the ring and the access waveguides. An improved design where a cantilever covers the curved portion of the race-track can potentially yield higher tunability range.

The rise time (t95) is the critical parameter which limits the dynamical performance of the device. From the measured resonance frequency and the extrapolated quality factor, approximate response time is calculated for two bimorph lengths using equation (5.2), which is shown in Table 5-IV. There is an unequal rise and fall times observed in the dynamic optical measurements which might be attributed by the softening of the cantilever during the voltage controlled actuation.

The ring resonator described here has a quality factor of $\sim 8000$ and a full width at half maximum of $\sim 195 \mathrm{pm}$. Within its free spectral range of $6.6 \mathrm{~nm}$, at least 16 dense wavelength division multiplexed (DWDM) channels of $0.4 \mathrm{~nm}(50 \mathrm{GHz})$ channel spacing can be realized. With the present results the device can be used as an on/off switch for a specific channel and by eliminating the stiction of the cantilever at pull-in, maximum tuning of few nanometers seems feasible considering the $5 \mathrm{~nm}$ shift obtained at pull-in. 
Table 5-IV. Rise time calculations for micro-cantilevers.

\begin{tabular}{|c|c|c|c|c|}
\hline $\begin{array}{c}\text { Cantilever } \\
\text { length } \\
(\mu \mathrm{m})\end{array}$ & $\begin{array}{c}\text { Resonance } \\
\text { frequency } \\
(\mathrm{kHz})\end{array}$ & $\begin{array}{c}Q \\
\text { (extrapolated from } \\
\text { Figure 5.8) }\end{array}$ & $\begin{array}{c}\text { Rise time } t_{95} \text { in }(\mu \mathrm{s}) \\
\text { (measured) }\end{array}$ & $\begin{array}{c}\text { Rise time } t_{95} \text { in }(\mu \mathrm{s}) \\
\text { (calculated using } \\
\text { equation }(0.2))\end{array}$ \\
40 & 805 & 1.7 & 2.5 (from Figure 5.12) & $2.03-3.38$ \\
100 & 140 & 1.8 & 16 (from Figure 5.28) & (With a fall time of 5) \\
\hline
\end{tabular}

\subsection{Conclusions}

Racetrack ring resonators with integrated micro-cantilevers have been fabricated successfully on SOI wafers and their mechanical and optical characterization has been carried out. Reversible resonance wavelength tuning of $122 \mathrm{pm}$ with a modulation depth of $18 \mathrm{~dB}$ is demonstrated for an integrated device. Maximum tuning can be larger $(>5$ $\mathrm{nm}$ ) when allowing pull-in and avoiding stiction by proper measures such as anti-stiction bumps or coatings. Further dynamic switching measurements are demonstrated out up to $30 \mathrm{kHz}$, with a 95\% signal rise time of $\sim 16 \mu \mathrm{s}$. Modulation at higher frequencies were limited by the signal rise time introduced by the detector used.

\subsection{References}

[1] H. V. Jansen, et al., MEMS-based nanotechnology, 2004.

[2] N. Tas, et al., "Stiction in surface micromachining," Journal of Micromechanics and Microengineering, vol. 6, pp. 385-397, 1996.

[3] K. Y. Yasumura, et al., "Quality factors in micron- and submicron-thick cantilevers," Journal of Microelectromechanical Systems, vol. 9, pp. 117 - 1252000.

[4] O. Solgaard, Photonic Microsystems: Micro and Nanotechnology Applied to Optical Devices and Systems: Springer, 2009.

[5] M. Bao and H. Yang, "Squeeze film air damping in MEMS," Sensors and Actuators A: Physical, vol. 136, pp. 3-27, 2007.

[6] K. B. Gavan, et al., "Resonance frequency behavior of silicon nitride cantilevers as a function of pressure in different gas environments," in IEEE Sensors, Christchurch, 2009, pp. $1836-1839$

[7] MEMSnet. Available: http://www.memsnet.org/material/

[8] P. Mela, et al., "Electrokinetic pumping and detection of low-volume flows in nanochannels," Electrophoresis, vol. 25, pp. 3687-3693, 2004.

[9] D. Taillaert, et al., "Grating couplers for coupling between optical fibers and nanophotonic waveguides," The Japan Society of Applied Physics, vol. 45, pp. 6071-6077, 2006.

[10] L. J. Kauppinen, "Compact integrated optical devices for optical sensor and switching applications," PhD Thesis, University of Twente, 2010. 
Chapter 5. Micro-cantilever integrated micro-ring resonator: Measurements 


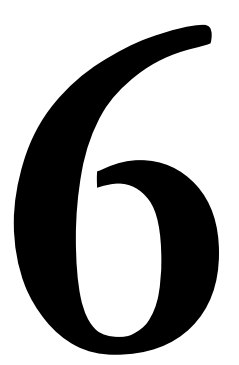

\title{
Photonic crystal slabs with integrated micro-cantilevers
}

\begin{abstract}
This chapter is divided into two sections: the first section presents a novel class of photonic devices which integrates micro-cantilevers with silicon 2-D photonic crystal slab waveguides. The second section presents a 2-D photonic crystal slab microcavity based device with integrated micro-cantilever. In both devices, microcantilevers are equipped with tips that are self-aligned relative to the holes of the photonic crystals. They modulate the waveguiding properties of the slab photonic crystal depending on the proximity of the tips to and into the holes. The integrated devices have been successfully fabricated and mechano-optically characterised.
\end{abstract}

*Part A of this chapter is submitted for publication as S.M.C. Abdulla, L.J. Kauppinen, M. Dijkstra, M.J. de Boer, E. Berenschot, R.M. de Ridder and G.J.M. Krijnen, "MEMS integrated 2-D photonic crystal slab waveguide for enhanced dispersion tuning".

*Part B of this chapter is accepted for publication in Journal of Micromechanics and Microengineering as S.M.C. Abdulla, L.J. Kauppinen, M. Dijkstra, M.J. de Boer, E. Berenschot, R.M. de Ridder and G.J.M. Krijnen, "Monolithically integrated cantilevers with selfaligned tips for wavelength tuning in a photonic crystal cavity based channel-drop filter". 
Chapter 6. Photonic crystal slabs with integrated micro-cantilevers

\section{Part A: Micro-cantilever integrated 2-D photonic crystal slab waveguide for enhanced dispersion tuning}

\subsection{Introduction}

Photonic crystals (PCs) are periodic dielectric materials having a photonic band gap; a frequency range for which light propagation is prohibited [1]. A row of missing holes in the PC constitutes a compact optical waveguide, with the possibility to create sharp bends with low optical loss. Having dynamic control over the PC waveguide characteristics allows for the realization of modulated optical devices such as switches, attenuators and tunable filters which can form the miniaturized photonic components for future integrated optical circuits.

Waveguiding properties of a photonic crystal slab waveguide (PCS-WG) can be controlled by a variety of methods such as thermo-optically [2], electro-optically [3], by means of liquid crystals [4], application of mechanical stress [5] or electromechanically [6]. Compared to these methods, modulation by an integrated electrostatic MEMS actuator has advantages such as compactness of the device, ease of integration, designable switching speed (trade-off between switching voltage and switching speed) and near-zero dissipation in the stationary state. Previous studies [7-9] have used atomic force microscopy (AFM) probes for mechanical tuning which requires tips of the AFM apparatus to be accurately aligned on a targeted PC hole. However, this method becomes impractical for cases which require more than one tip to be inserted in selected PC holes. In [6], a micro-cantilever integrated PCS-WG, fabricated on a silicon-on-insulator (SOI) wafer, demonstrating electromechano-absorptive modulation at $10 \mathrm{~Hz}$ with a $0-60 \mathrm{~V}$ square-wave excitation was reported. The measured rise and fall times of this device are $850 \mu$ s and $1.5 \mathrm{~ms}$ respectively. The operation of the device is based on dielectric loading of the PCS-WG with a poly-silicon beam having a higher refractive index than silicon, which on actuation causes the guided light to leak into the beam.

The transmittance of a 2D PCS-WG is defined by the refractive index of the slab and the air hole geometry such as lattice constants, the shapes and the sizes of the air holes. The photonic band structure of this PCS-WG can be mechanically modulated by inserting tips that have a refractive index higher than that of the air hole. The aim is to develop a process for fabricating protrusions (tips) on cantilevers, such that the tips are self-aligned to the holes of a previously fabricated photonic crystal and electrostatic actuation of the cantilever will move the tips into the holes.

High-precision fabrication technology is essential for the realization of on-chip devices in which tips are accurately aligned to the PC holes. Filling holes of an SOI-based photonic crystal defect waveguide by an electrostatically actuated poly-silicon cantilever 
that has silicon rods at the end has been demonstrated in [10]. In a different approach, a MEMS-actuated cantilever has been integrated with a PC-membrane device which inserts defect posts into PC holes [11]. Though this device has been successfully fabricated, to our knowledge no optical modulation has been reported so far. A broadband optical switch, where a flexible SOI-based PCS-WG is electrostatically deflected to accept an array of protruding nanorods in its holes is presented in [12]. Fabrication of the devices [11-12] requires direct-writing electron beam lithography which is quite laborious and prohibitively expensive for large scale fabrication.

SOI-based air bridge PCS-WGs have two distinct properties: a high index contrast which enables strong in-plane confinement, and a symmetric cladding which prevents coupling between the TE-like and TM-like modes. Also the air light line is higher than the silica light line, which increases the operating bandwidth of the device. However its membrane-like geometry makes it difficult for post processing and monolithic integration with mechanical or electrical structures. In this chapter we report two important achievements: (i) fabrication and monolithic integration of a novel class of devices with electrostatically actuated bimorph cantilevers having self-aligned tips and (ii) transforming an initially asymmetric PCS-WG into a symmetric one by locally removing the under cladding, thus creating an air bridge structure. The integration is performed on wafer scale, using surface micromachining techniques where each air hole of the PCS-WG has a silicon-rich nitride $\left(\mathrm{SiN}_{x}\right)$ tip self-aligned on top of it. The bimorph cantilevers, equipped with tips, can change the propagation properties of the photonic crystal slab depending on the proximity to the holes. Since in high optical index contrast structures the exponentially decaying evanescent fields extend typically only a few hundred nanometres into the air cladding, the required cantilever strokes for switching can be accordingly small. This implies that for fast actuation, stiff cantilevers with resonance frequencies in the $\mathrm{MHz}$ range can be used, while still requiring only moderate actuation voltages. The integrated bimorph discussed here consists of an upper layer, which acts as the electrode, and a dielectric lower layer. Stress, induced by the different thermal expansion coefficients of the layers that were deposited at elevated temperatures, makes the bimorph bend upward in the off-state (see section 3.5.2). The bimorph is actuated by application of a voltage between the top electrode and the substrate. As a proof of concept, optical modulation of the integrated device is demonstrated.

The organization of this section of the chapter is as follows. The next section briefly describes the fabrication technology for integrating the bimorph cantilever with the PCS-WG. The mechanical characterization of the integrated device is presented in section 6.4 whereas the optical characterisation is presented in section 6.5. Finally section 6.6 provides a summary and conclusions. 


\subsection{Fabrication}

\subsubsection{Optical device fabrication}

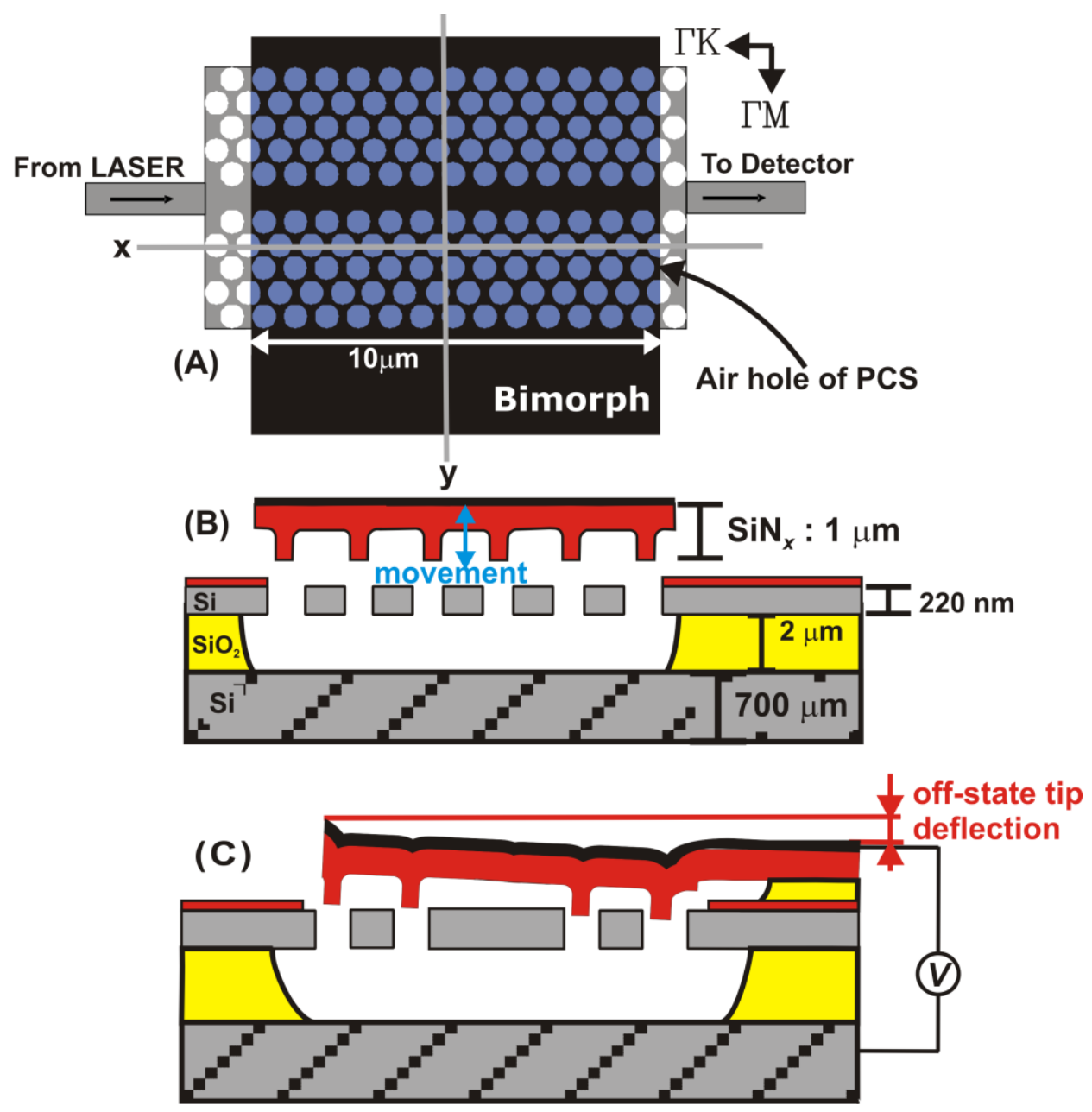

Figure 6.1. Schematic illustration of the integrated device. (A) Top view, (B) cross-sectional view through $x$-direction and $(C)$ cross-sectional view through $y$-direction.

Figure 6.1 shows a schematic illustration of the integrated device with (A) top view and (B), (C) cross-sectional views along the $x$ and $y$ directions. PCS-WGs and their access waveguides are fabricated on SOITEC SOI wafers using deep-UV lithography by the silicon photonics platform ePIXfab [13]. The thickness of the silicon device layer (i.e. the PC slab thickness) is $220 \mathrm{~nm}$ and that of the handle wafer is $700 \mu \mathrm{m}$. Before integration, the lower cladding is a $2 \mu \mathrm{m}$ thick thermal oxide (refractive index, $n=1.449$ at $1550 \mathrm{~nm}$ ) and the upper cladding is air $(n=1.0)$. The final integrated device has lower and upper claddings as air. The PCS-WG, designed to operate in the C-band $(1530-1565 \mathrm{~nm})$ of the telecommunication wavelengths, has a designed hole diameter of $270 \mathrm{~nm}$ and a lattice constant of $440 \mathrm{~nm}$. The W1 waveguide in the photonic crystal is formed by a line defect resulting from the removal of a single row in the $\Gamma \mathrm{K}$ direction. 


\subsubsection{Micro-cantilever integration}

Figure 6.2 shows the fabrication flow for the integrated device. (a) PCS-WGs, are fabricated on a $200 \mathrm{~mm}$ SOI wafer by a $193 \mathrm{~nm}$ deep-UV lithography process at ePIXfab. The wafer is then diced into four pieces to fit into the $100 \mathrm{~mm}$ wafer systems of the $\mathrm{MESA}^{+}$clean room. (b) On top of these wafers, first a $43 \mathrm{~nm}$ thick low pressure chemical vapour deposition (LPCVD) [14] low-stress $\mathrm{SiN}_{x}$ layer is deposited which acts as a protective layer (PL) for the access waveguides during a later sacrificial layer etching (SLE) step. This layer is removed from the backside of the wafer by reactive ion etching (RIE) to later have electrical access to the handle wafer to function as the lower electrode. In order to fabricate tips and to reduce optical losses, the protective nitride has to be removed from the PC area of the wafer. For this reason, (c) a $93 \mathrm{~nm}$ thick tetra-ethylortho-silicate layer (TEOS oxide, at $700^{\circ} \mathrm{C}$ and $400 \mathrm{mTorr}$ ) is deposited as an etch-mask layer. A (d) dark mask (OiR 907-17, Fujifilm, thickness of resist $=1.7 \mu \mathrm{m}$ ) is used to define the etch windows in the PC area. After the lithography, the wafer is first ozone treated to make the $\mathrm{SiO}_{2}$ (TEOS) layer hydrophilic and then chemically etched in BHF (buffered hydrofluric acid, $\mathrm{NH}_{4} \mathrm{~F}: \mathrm{HF}=7: 1$ ) to remove the $\mathrm{SiO}_{2}$ (TEOS) from the etch windows. (e) After stripping the photoresist in $99 \% \mathrm{HNO}_{3}$ for 20 minutes, the PL is removed from the etch window by $85 \% \mathrm{H}_{3} \mathrm{PO}_{4}$ at $180^{\circ} \mathrm{C}$. (f) Following this, the SOI wafers are first coated conformally by a $105 \mathrm{~nm}$ thick $\mathrm{SiO}_{2}$ (TEOS) sacrificial layer (SL)

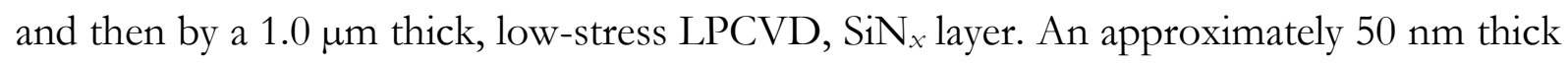
gold layer $(\mathrm{Au})$ is sputtered as the top electrode layer. A thin layer of chromium (Cr) is used as an adhesive layer for gold where the thickness of $\mathrm{Cr}$ is selected to be as low as possible, to limit the stress in the bimorphs. The measured total thickness of the electrode layer $(\mathrm{Au}+\mathrm{Cr})$ by Dektak-V.8 surface profiler is $58 \mathrm{~nm}$. 
Chapter 6. Photonic crystal slabs with integrated micro-cantilevers

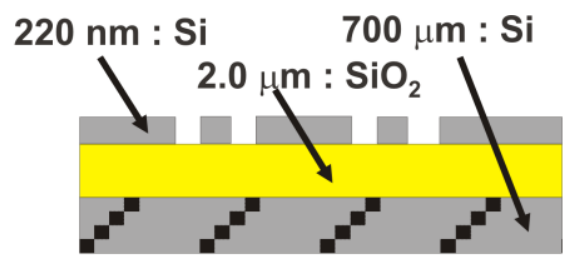

(a) SOI wafer in which PCS-WG is fabricated

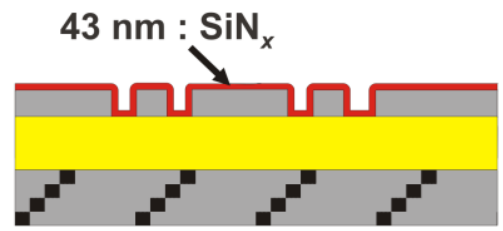

(b) Deposition of protective - $\mathrm{SiN}_{x}$ and dry etching from backside

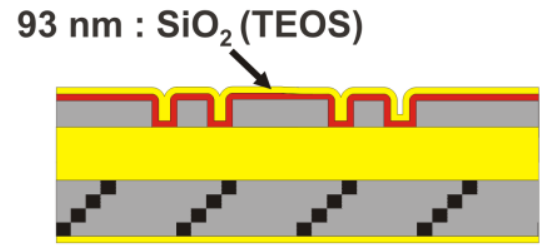

(c) Deposition of TEOS etch mask layer

(d) Patterning and wet etching of TEOS

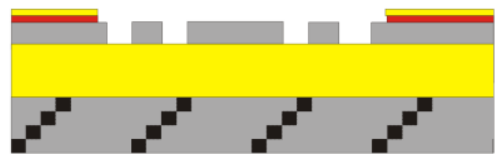

(e) Wet etching of protective $\mathrm{SiN}_{x}$ layer
$105 \mathrm{~nm}: \mathrm{SiO}_{2}$ (TEOS)

$58 \mathrm{~nm}: \mathrm{Au}+\mathrm{Cr} \backslash 1.0 \mu \mathrm{m}: \mathrm{SiN}_{x}$

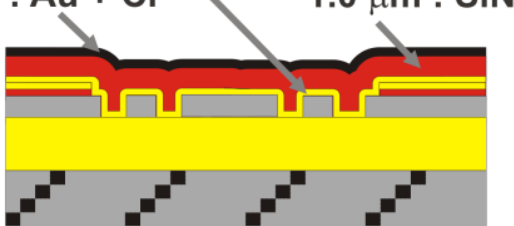

(f) Deposition of TEOS SL, $\operatorname{SiN}_{x}$ (bimorph) and sputtering of $\mathrm{Cr}-\mathrm{Au}$ layers

Mask 2

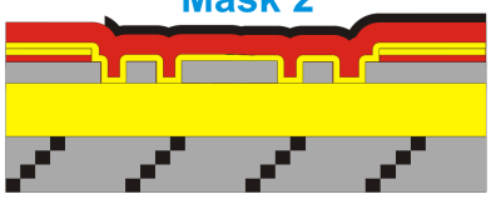

(g) Wet etching of patterned $\mathrm{Cr}-\mathrm{Au}$ layers

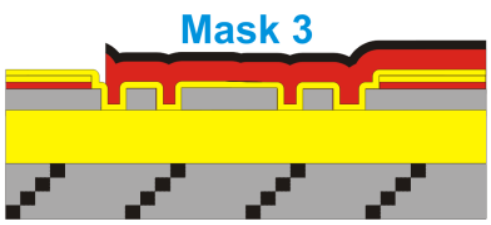

(h) Dry etching of patterned $\mathrm{SiN}_{x}$ layer

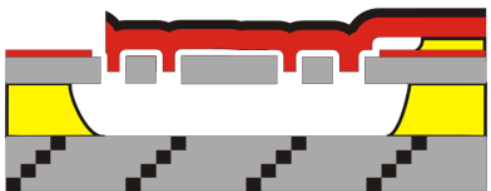

(i) Sacrificial layer etching

Figure 6.2. Fabrication flow for integrating micro-cantilevers having self-aligned tips with the PC Slab.

The second mask is used for $(\mathrm{g})$ pattering the metal layers using OiR 907-17 resist as etchmask. Subsequently $\mathrm{Au}$ is etched in gold etch solution at $30^{\circ} \mathrm{C}(\mathrm{KI}=132 \mathrm{~g}, \mathrm{I} 2=18 \mathrm{~g}$, DI $=1200 \mathrm{ml}$, in which glycerin $(600 \mathrm{ml})$ is added to reduce excessive undercut). Followed by this, $\mathrm{Cr}$ is wet etched in chromium etchant (MERCK 111547.2500). (h) The third mask is used for patterning the $\mathrm{SiN}_{x}$ device layer of the bimorph, using a resist mask (OiR 90835 , Fujifilm, thickness of resist $=3.5 \mu \mathrm{m}$ ). This layer is etched from front and back sides by RIE (Elektrotech PF340 at $10^{\circ} \mathrm{C}, 75 \mathrm{~W}, 10 \mathrm{~m}$ Torr, $25 \mathrm{sccm} \mathrm{CHF}_{3}$ and $5 \mathrm{sccm} \mathrm{O}_{2}$ ). After this, the resist is first etched for 1 minute in $\mathrm{O}_{2}$ plasma etcher (TEPLA 300E at 500 $\mathrm{W}, 1.2 \mathrm{mbar}$, and $200 \mathrm{sccm}_{2}$ ) for removing the possible fluorocarbon contamination produced by RIE etching. Subsequently, resist stripping is continued in $\mathrm{HNO}_{3}(99 \%)$ for 
20 minutes. Finally (i) SLE is done by BHF $\left(\mathrm{NH}_{4} \mathrm{~F}: \mathrm{HF}=7: 1\right)$ followed by freeze drying [15] (see also Appendix I) to release the cantilevers. As explained in section 4.4.2.4, the SLE is divided into three steps in which an ultrasonic cleaning step is included to remove the $\operatorname{SiN}_{x}$ residues which are called stringers. The fabricated cantilevers have lengths varying from $40 \mu \mathrm{m}$ to $100 \mu \mathrm{m}$ at a fixed width of $10 \mu \mathrm{m}$ for all cases. A detailed fabrication flow of this process is presented in Appendix II-Table III.

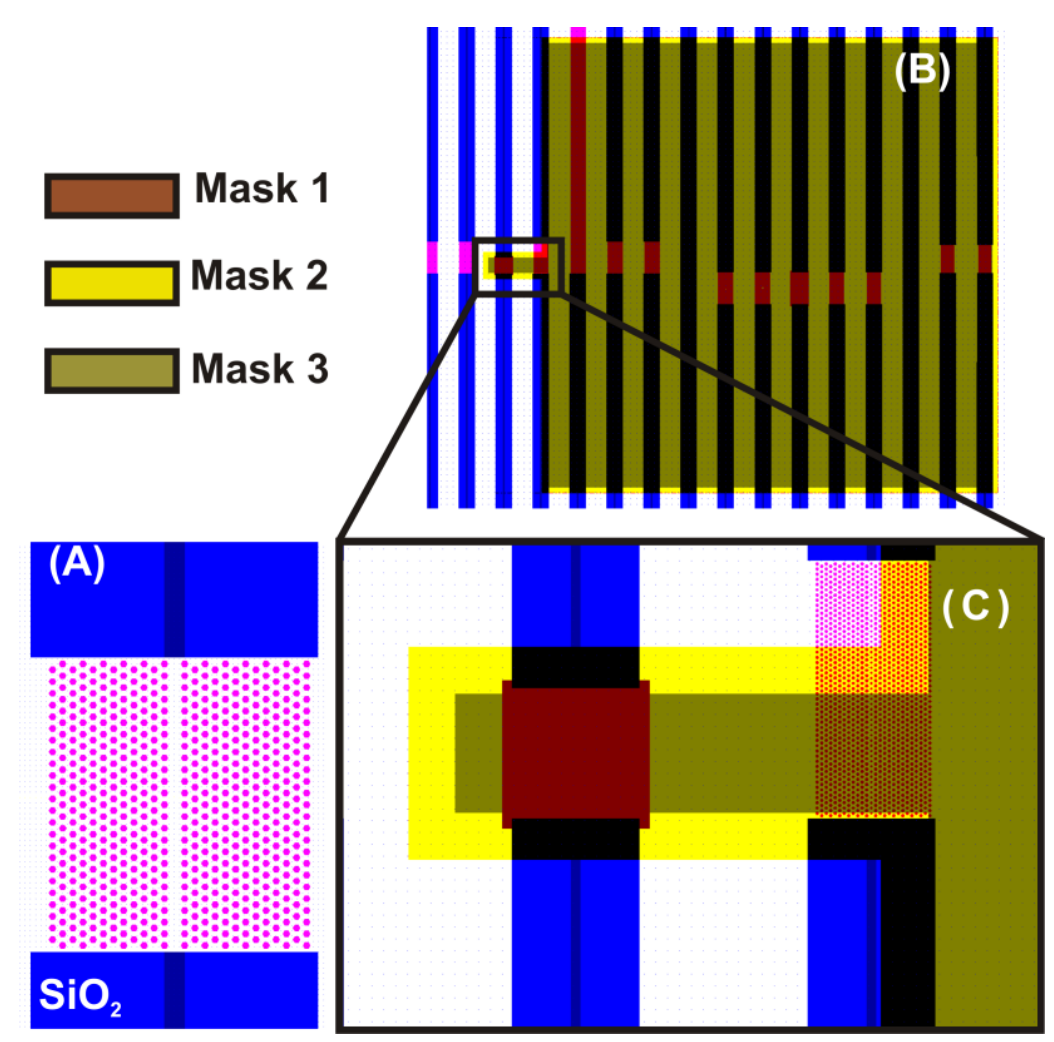

Figure 6.3. The mask layout: (A) for the PCS-WG and $(B, C)$ for the micro-cantilever integration.

The fabrication is a three-mask process where all the masks use positive photoresist for the UV lithography. The mask layout used for the PCS-WG fabrication and the microcantilever integration are shown in Figure 6.3 1. The first mask is used (Figure 6.2d) to open the $\mathrm{SiN}_{x}$ on the PCS-WG areas. The second mask is used (Figure 6.2g) for pattering the metal layers and the third mask is used (Figure 6.2h) for patterning the $\mathrm{SiN}_{x}$ bimorph device layer. Note that none of the masks are critically aligned to the holes of the PCS-

${ }^{1}$ Mask 2 is designed larger than Mask 3, since the process contains a variation in cantilever design as well which is not described in this thesis. This design includes patterning of metal layer on top of a cantilever which includes features as small as $2 \mu \mathrm{m}$. Hence to avoid the misaligned between mask 2 and mask 3, mask 2 is made larger than mask3. However the device geometries are defined by mask 3. A metal etching is carried out after doing lithography using mask 2 and mask3. However for the PCS-WGs described in this chapter, mask 2 can be avoided as well. 
WG. Figure 6.4A shows a zoomed-out SEM image of the integrated device with its access waveguides and Figure 6.4B, the self-aligned tips in the off-state of the cantilever. In Figure 6.3 and Figure 6.4A it can be seen that the cantilever is integrated above two PCSWGs. However the characterisation carried out in this chapter is performed only on the PCS-WG which is below the tip area of the cantilever. The PCS-WG near the anchor area of the cantilever is not used for any of the measurements.
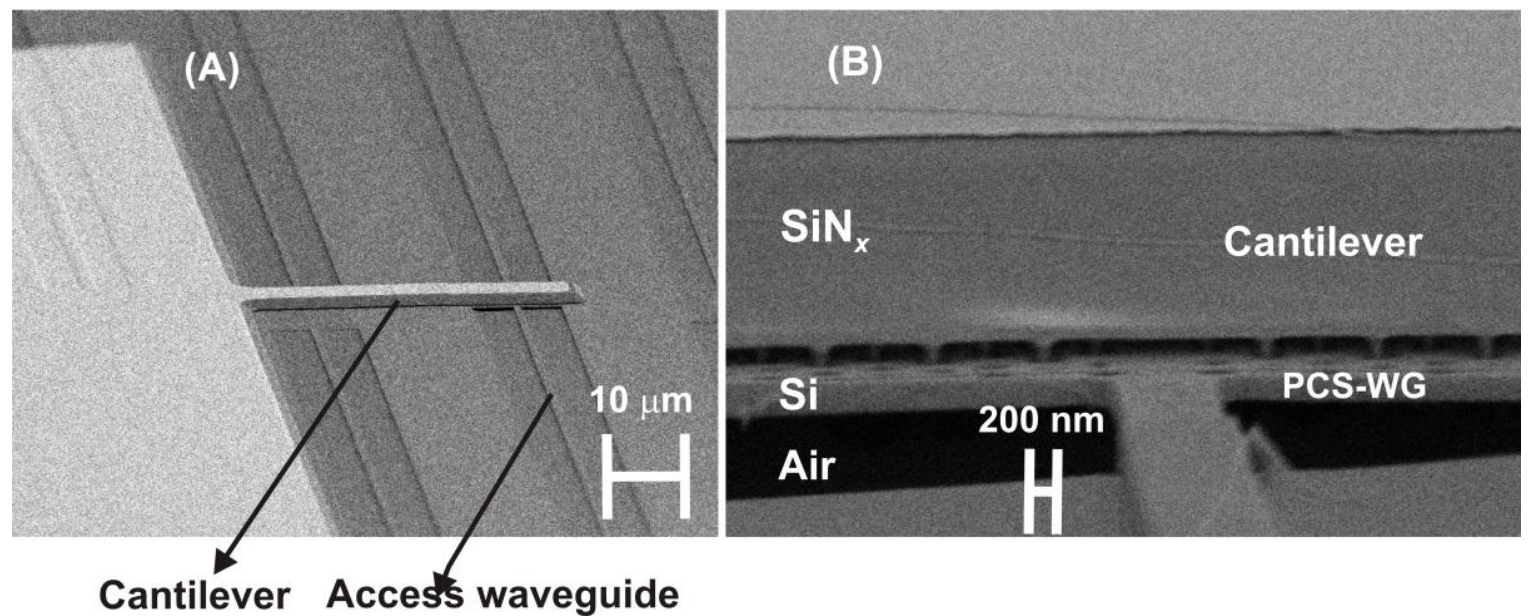

Figure 6.4. SEM images of the integrated device (A) overview and (B) details of the tips self-aligned with respect to the holes of the PCS-WG.

Timed etching of the SL also defines the anchor region of the cantilever, which provides a convenient bond pad area of $300 \times 300 \mu \mathrm{m}$. The actual length of the fabricated bimorph is increased from the design length due to the undercut at the bond pads caused by the SLE. The undercut, as measured with an optical microscope is $5.5 \mu \mathrm{m}$. This increased effective bimorph length results in a reduced resonance frequency and pull-in voltage, as explained in section 3.6.

\subsection{Discussions on fabrication}

The first part of this section provides a detailed discussion on the material selection for the micro-cantilever integration process. Section 6.3.2 discusses the effects of conformal layer deposition on self-aligned tip formation, on PC holes and on the bimorph cantilever. The photochemical etching of silicon in BHF is discussed in section 6.3.3.

\subsubsection{Material selection}

The material and thickness of each layer for the integrated device were chosen carefully, considering various design parameters. Table 6-1 shows the measured variation in thickness across the wafer and the measured refractive index for various conformal layers used for the fabrication. Owing to its high uniformity and good conformal step coverage, LPCVD $\mathrm{SiO}_{2}$ (TEOS) was chosen as the SL. It is important to protect the thermal oxide 
lower cladding layer beneath the access waveguides during SLE. If unprotected, during SLE it will be attacked by BHF. This will result in releasing the access waveguides thereby destroying the devices. The PL was selected to be $\mathrm{SiN}_{x}$ because of its high resistance (etch rate $0.5 \mathrm{~nm} / \mathrm{min}$ ) to the $\mathrm{BHF}$ etchant used for SLE of $\mathrm{SiO}_{2}$ (TEOS, $180 \mathrm{~nm} / \mathrm{min}$ ). The thickness of the PL is selected in such a way that during SLE, it is not completely etched and still acts as a thin protective layer for the access waveguides of the PCS-WG. The required SL etch time of the $10 \mu \mathrm{m}$ wide cantilever $(5000 / 150 \sim 33 \mathrm{~min})$ defines the minimum thickness of the PL to be approximately $17 \mathrm{~nm}(33 \times 0.5)$. However an overetching of 19 minutes is performed to ensure that the $\mathrm{SiO}_{2}$ beneath the cantilever is completely removed. Including this extra time, and a safety time to counteract delays caused by layer non-uniformity, a PL thickness of $43 \mathrm{~nm}$ is selected. Increasing its thickness will affect the optical properties of the access waveguides (they may become multimodal) and therefore the PL has to be as thin as possible, but still thick enough to sustain the SLE. In this process after the SLE, the thickness of the remaining protective $\mathrm{SiN}_{x}$ layer is $<20 \mathrm{~nm}$.

Table 6-1. Measured thickness variation across the wafer and refractive indices of the conformal layers.

\begin{tabular}{|c|c|c|c|}
\hline Layer (function) & $\begin{array}{c}\text { Thickness } \\
\text { (nm) }\end{array}$ & $\begin{array}{c}\text { Standard deviation }(3 \sigma) \\
(\mathrm{nm})\end{array}$ & $\begin{array}{c}\text { Ellipsometer } \\
\text { measurement showing } \\
\text { refractive index (n) at } \\
633 \mathrm{~nm}\end{array}$ \\
\hline $\mathrm{SiN}_{x}$ (Protection) & 43 & 2 & 2.20 \\
\hline $\mathrm{SiO}_{2}$ TEOS (Etch-mask) & 93 & 1 & 1.45 \\
\hline $\mathrm{SiO}_{2}$ TEOS (Sacrificial) & 105 & 1 & 1.44 \\
\hline $\operatorname{SiN}_{x}$ (Cantilever) & 1061 & 41 & 2.18 \\
\hline
\end{tabular}

In order to observe any mechano-optical modulation upon electrostatic actuation, the tips of the cantilever have to be slided into the PCS-WG holes. A large air gap between the cantilever and the PCS-WG will require a higher voltage to pull it towards the substrate. In this technology, a large gap would also eliminate the possibility to fabricate self-aligned tips. The vertical air gap between the cantilever and the PCS-WG is mainly determined by the thickness of the SL. The smallest possible thickness of the SL for this process is related to the etch-selectivity between $\mathrm{SiN}_{x}$ and $\mathrm{SiO}_{2}$ (TEOS) in RIE (Figure 6.2h) and the non-uniformity in both layers. The etch rate of $\mathrm{SiO}_{2}$ (TEOS) in RIE (at $10^{\circ} \mathrm{C}, 75 \mathrm{~W}$ power, $10 \mathrm{mTorr}$ pressure) is $35 \mathrm{~nm} / \mathrm{min}$ and that of $\mathrm{SiN}_{x}$ is $55 \mathrm{~nm} / \mathrm{min}$, hence a limited selectivity of $\mathrm{SiN}_{x}: \mathrm{SiO}_{2}$ (TEOS) = 1.6:1. For larger non-uniformity of the layers, the selectivity of the etching process will limit the maximum thickness ratio of the $\mathrm{SiN}_{x}$ 
Chapter 6. Photonic crystal slabs with integrated micro-cantilevers

cantilever layer and the $\mathrm{SiO}_{2}$ (TEOS) SL to a lower value. In this design, the $\mathrm{SiN}_{x}$ cantilever layer should be selected to be sufficiently thick in order to prevent optical loss caused by the interaction of the evanescent optical field with the conducting (i.e. optically lossy) $\mathrm{Au}$ electrode. Considering the aforementioned limited selectivity and non uniformity of the $\mathrm{SiN}_{x}$ layer, the SL thickness is selected to be approximately $100 \mathrm{~nm}$. The measured thickness of the deposited SL is $105 \mathrm{~nm}$. Moreover, the SL thickness has to be small enough to fabricate the self-aligned tips, as will be explained in the next section. By carefully checking the thickness and non-uniformity in both layers with dummy wafers and by analyzing the loading effect, we could successfully etch through a $1.0 \mu \mathrm{m} \mathrm{SiN}$ layer on top of a $105 \mathrm{~nm} \mathrm{SL}$. Gold was chosen as the top electrode due to its relatively low residual stress compared to chromium, which reduces the upward bending of the cantilever (see section 3.5.2). A combination of an approximately $50 \mathrm{~nm}$ thick Au layer and an $8 \mathrm{~nm}$ thick Cr layer yields an acceptable off-state deflection $(20 \mathrm{~nm})$ for this device.

\subsubsection{Effects on conformal deposition}

Conformal layers of high uniformity are essential for a successful fabrication of this novel integrated device. Except the metal, all other layers in this process are deposited by LPCVD. The important features of a LPCVD conformal layer are the void free filling, excellent step coverage and rounding on sharp corners. This section discusses the shape of the fabricated tips, the remaining material in the corners of the PC hole and the stepped cantilever beam which are important effects realized by the conformal layer deposition.

\section{Self-aligned tip}

The height of the fabricated tips is related to the thickness of the SL and the hole diameter. For a hole diameter of $270 \mathrm{~nm}$, an increase in the SL-thickness $\left(t_{\mathrm{SL}}\right)$ decreases the height of the tips (Figure 6.5-(2)). In this case, for $t_{\mathrm{SL}}<135 \mathrm{~nm}$ (half of the PC hole diameter), the tip height is approximately constant at $220 \mathrm{~nm}$ (Figure 6.5a,b-(2)). For $t_{\mathrm{SL}}>$ $135 \mathrm{~nm}$, the SL completely fills the $270 \mathrm{~nm}$ holes which results in tips whose height is less than $50 \mathrm{~nm}$ (Figure 6.5c-(2)). Increasing $t_{\mathrm{SL}}$ further will result in a flat cantilever having no tips (Figure 6.5d-(2)). Hence for fabricating tips into a hole of diameter $270 \mathrm{~nm}$, the SLthickness should be less than $135 \mathrm{~nm}$. The actual hole diameter depends on the lithographic exposure dose. For fabricating the PC holes an exposure dose sweep is performed and due to it the hole diameter (as measured) ranges $\pm 20 \mathrm{~nm}$ around the designed value [22]. From Figure 6.5a(1-3) it is clear that an increase in hole diameter 
increases the width of the tips for a given SL thickness. Considering these considerations, in this case, an optimum SL-thickness of $100 \mathrm{~nm}$ (Figure 6.5b-(2)) was selected.
(1)
(2)
(3)

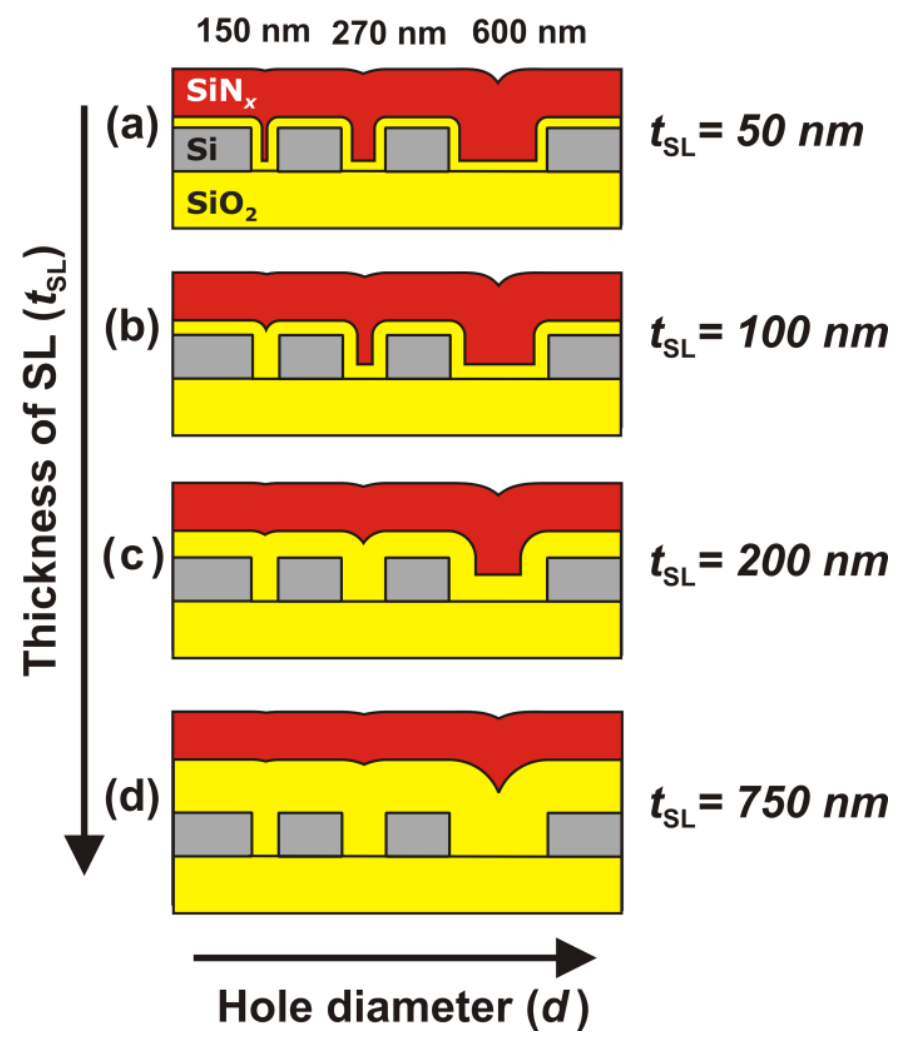

Figure 6.5. Effect of self aligned tip on sacrificial layer thickness and hole diameter.

From the high-resolution-SEM images, it is observed that the side walls of the PC holes are not exactly at right angles but at an obtuse angle $\left(<10^{\circ}\right)$. This geometry, together with the conformal deposition results in tips having a conical shape as shown in Figure 6.6b.

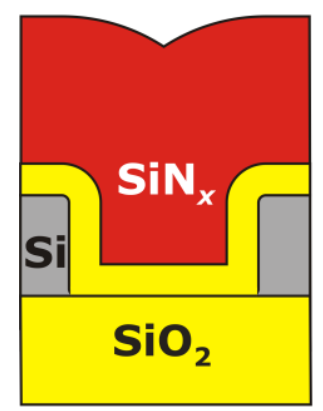

(a)

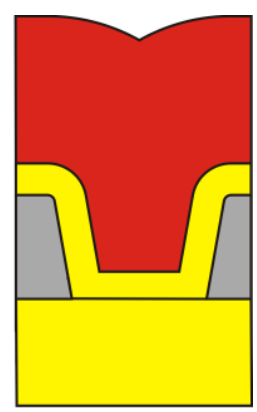

(b)

Figure 6.6. Effect of a tapered PC hole on the shape of the tip.

Figure 6.7 shows the SEM images of a tip partially inserted into a PC hole (A) and when the PCS-WG is locally removed from the wafer (B). In this kind of SEM imaging, the 
charge deposited by the electron beam on the insulated cantilevers causes the cantilevers to be attracted towards the conducting Si PCS-WG.

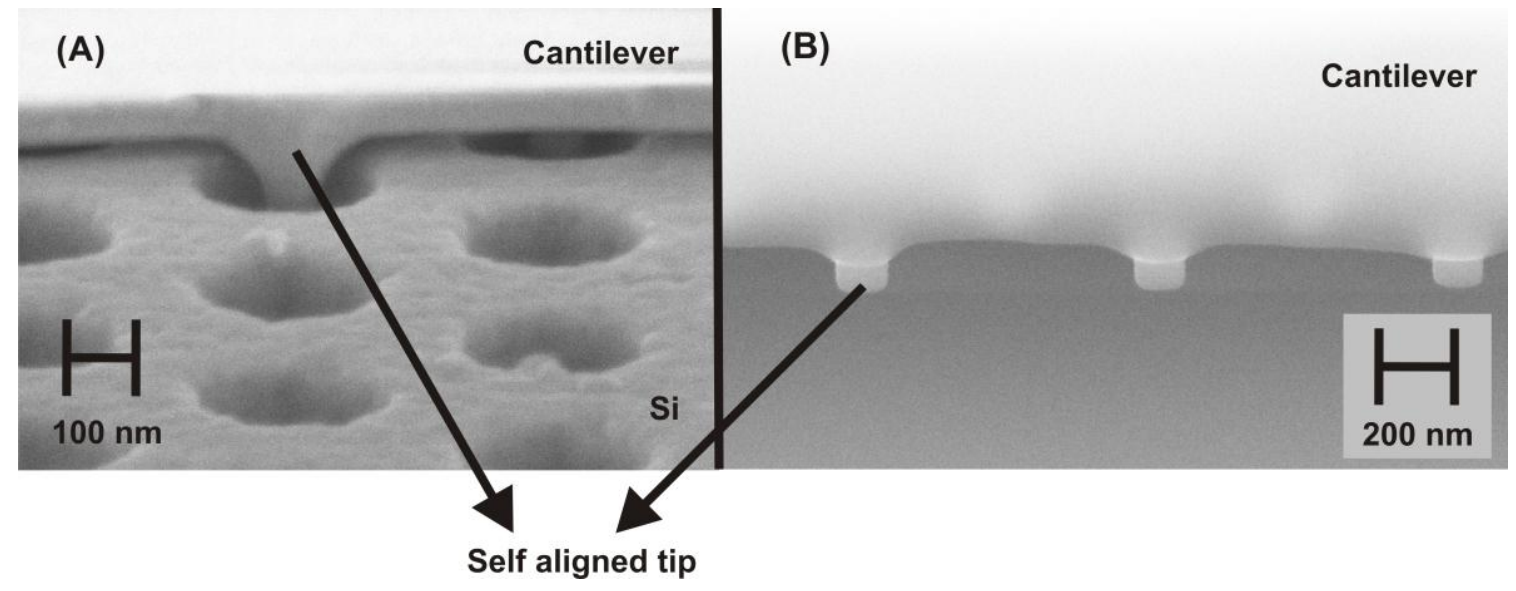

Figure 6.7. Oblique view of a self-aligned tip (A) inserted into a PC hole and (B) when the PCS-WG is locally removed from the wafer.

\section{$>$ Photonic crystal holes}

Conformal step coverage of the PC holes by the PL and the subsequent isotropic etching leaves the corners of the holes with residual materials called, stringers (Figure 6.8 and also see section 4.4). The dispersion properties of the photonic crystal are strongly related to the PC hole dimensions, lattice constant and the materials. If the thickness of the stringers exceeds a critical value, their presence can destroy the PC properties. Hence, it is important to remove the stringers from the holes, which can be realised by overetching of the layers. A hole of $270 \mathrm{~nm}$ diameter is completely closed if a PL thickness of $43 \mathrm{~nm}$ and a $\mathrm{SiO}_{2}$ (TEOS) etch-mask layer of $\geq 92 \mathrm{~nm}$ (here the measured thickness is $93 \mathrm{~nm}$ ) are deposited (Figure 6.8a). In order to open this hole again, it is needed to etch $313 \mathrm{~nm}$ $(220+93)$ of $\mathrm{SiO}_{2}$ (TEOS) layer in $\mathrm{BHF}$ (Figure 6.8b). The etch rate of $\mathrm{SiO}_{2}$ (TEOS, not annealed) in $\mathrm{BHF}$ is $180 \mathrm{~nm} / \mathrm{min}$. With a 2 min overetch it is sure that the stringers arising from the $\mathrm{SiO}_{2}$ (TEOS) etch-mask layer are completely removed from the holes (Figure 6.8c).

The next step is to etch the PL from the PC holes. The measured selectivity as found from the etch rates by $85 \% \mathrm{H}_{3} \mathrm{PO}_{4}$ at $180^{\circ} \mathrm{C}$ was $\mathrm{SiN}_{x}: \mathrm{SiO}_{2}(\mathrm{TEOS})=1.8: 1$. This poor selectivity restricts the overetching of the PL from the holes, which leaves $\mathrm{SiN}_{x}$ stringers at the base of the holes (Figure 6.8d). In this case after hot $\mathrm{H}_{3} \mathrm{PO}_{4}$ etching, the thickness of the remaining $\mathrm{SiO}_{2}$ (TEOS) etch-mask layer is $49 \mathrm{~nm}$. One way to improve the etch selectivity is by annealing the etch-mask layer. However, exposing silicon in hot $\mathrm{H}_{3} \mathrm{PO}_{4}$ for a long time could increase its surface roughness which could introduce optical loss. 
Hence overetching has to be restricted to as small as possible in order to maintain the quality of the silicon layer.
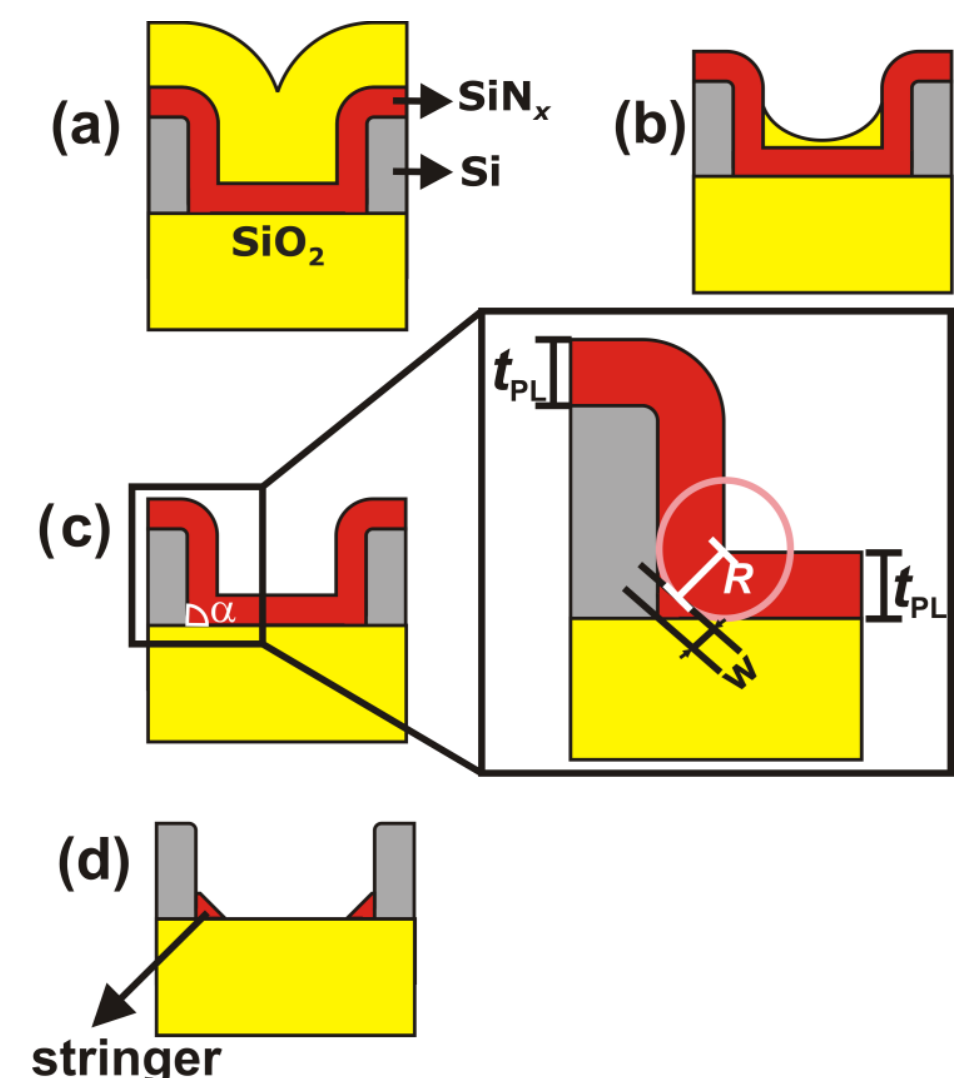

Figure 6.8. Schematic representation of a single PC hole during the isotropic wet etching steps. Step ' $d$ ' shows the stringers left on the corner of the hole.

The width 'w' of the stringer is given by the relation [16],

$$
w=\frac{t_{P L}}{\sin \left(\frac{\alpha}{2}\right)}-R
$$

Where 'tPL' is the thickness of the PL, $\alpha$ is the corner angle and R is the isotropic etch distance (Figure 6.8c). As $\alpha$ is varying slightly along the wafer from hole to hole, the width of the stringer also slightly varies. This means, as $\alpha / 2$ ranges between 45 and 50 degrees, the width of the stringer also varies between 17.8 and $13.2 \mathrm{~nm}$.

For an investigation on the shape of the $\mathrm{SiN}_{x}$ stringers inside the hole, one of the PC's was milled by focused ion beam (FIB) soon after the PL was removed (step ' $e$ ' of Figure 6.2). After milling (Figure 6.9A), it was etched for 2 min using 1\% HF to partially release the stringers. This showed the stringers to be in ring shape (Figure 6.9B). Further, a partial etch of silicon in $25 \%$ tetra-methyl ammonium hydroxide (TMAH) at $70^{\circ} \mathrm{C}$ for 2 min, clearly confirmed the circular shape of the stringers (Figure 6.9C). In order to assess the optical loss introduced by these $\mathrm{SiN}_{x}$ rings, we have compared the transmission of 
light through a straight waveguide (length $=6 \mathrm{~mm}$ and width $=600 \mathrm{~nm}$ ) without integrated cantilever and the PCS-WG with integrated cantilever (in off-state). The losses in both waveguides are less than $2 \mathrm{~dB}$ [17] indicating that the $\mathrm{SiN}_{x}$ rings inside the holes do not introduce large optical losses.
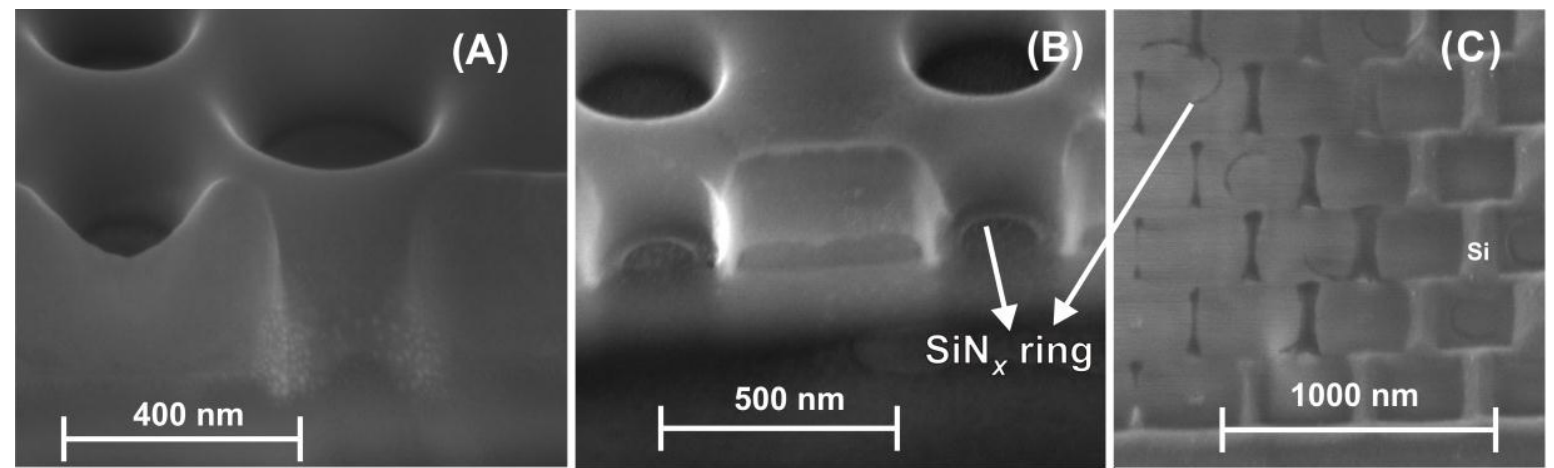

Figure 6.9. Cross-sectional view of $S i N x$ rings inside the PC boles after FIB milling (A), after etching in $1 \% \mathrm{HF}$ for $2 \mathrm{~min}(\mathrm{~B})$ and after partially etching silicon in $25 \% \mathrm{TMAH}$ at $70^{\circ} \mathrm{C}$ for $2 \mathrm{~min}(\mathrm{C})$.

In order to investigate the etching of the lower $\mathrm{SiO}_{2}$ cladding layer (during SLE), a cantilever is removed from one of the integrated devices after the freeze drying release step. Figure 6.10A shows the part of the PCS-WG membrane which is broken apart and the inset, Figure $6.10 \mathrm{~B}$ shows a magnified view of the ring-shaped $\mathrm{SiN}_{x}$ stringers at the bottom of the PC holes.

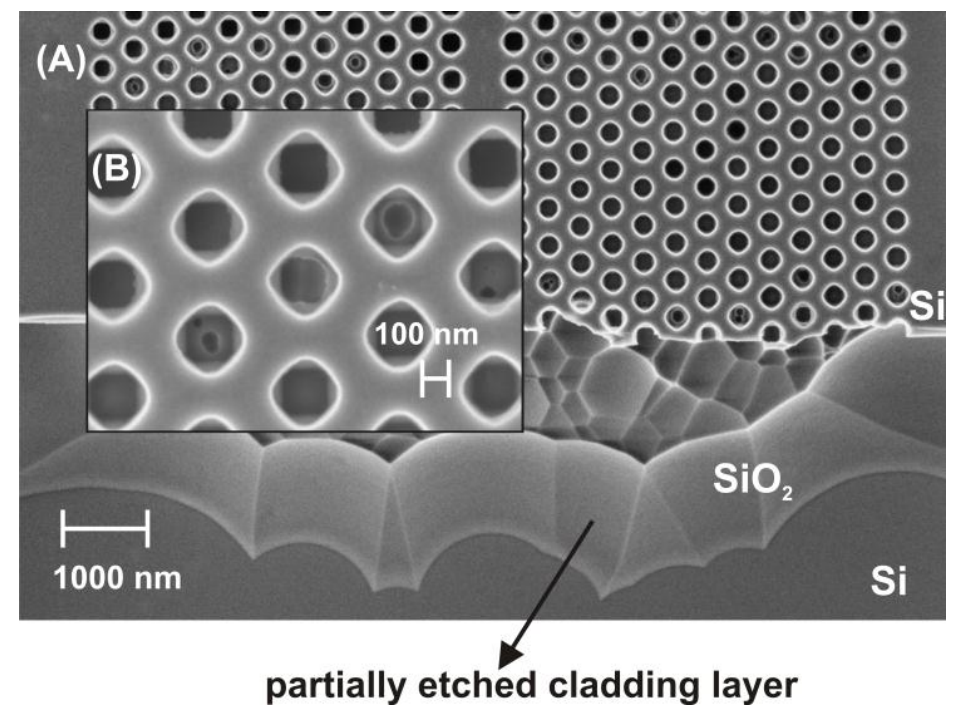

Figure 6.10. SEM pictures of $(A)$ top view of $P C$ boles after removing the integrated cantilever and inset (B) SiN $\mathbf{x}$ ring-shaped stringers inside the holes

To account for misalignment during lithography, the etch window (mask 1) was chosen larger than the PCS-WG area (Figure 6.11A). This creates larger etch holes around the etch window through which, during SLE, the lower cladding is etched faster than through 
the PC holes. Moreover, the SL (below the cantilever) is exposed to BHF in an increased time near the sides of the cantilever relative to the centre. Due to this, the diffusion of BHF (through the PC holes) below the centre of the cantilever starts only at the final stages of the SLE. These reasons make that for some devices, the lower $\mathrm{SiO}_{2}$ cladding to be only partially etched below the centre of the cantilever as shown in Figure 6.10A. The lower cladding below the PC area can be completely removed by increasing the SLE time, but this in turn can pose a risk for the thin PL. Hence in this process, the SLE overetching was limited to 19 minutes in addition to the required SLE time of 33 minutes.
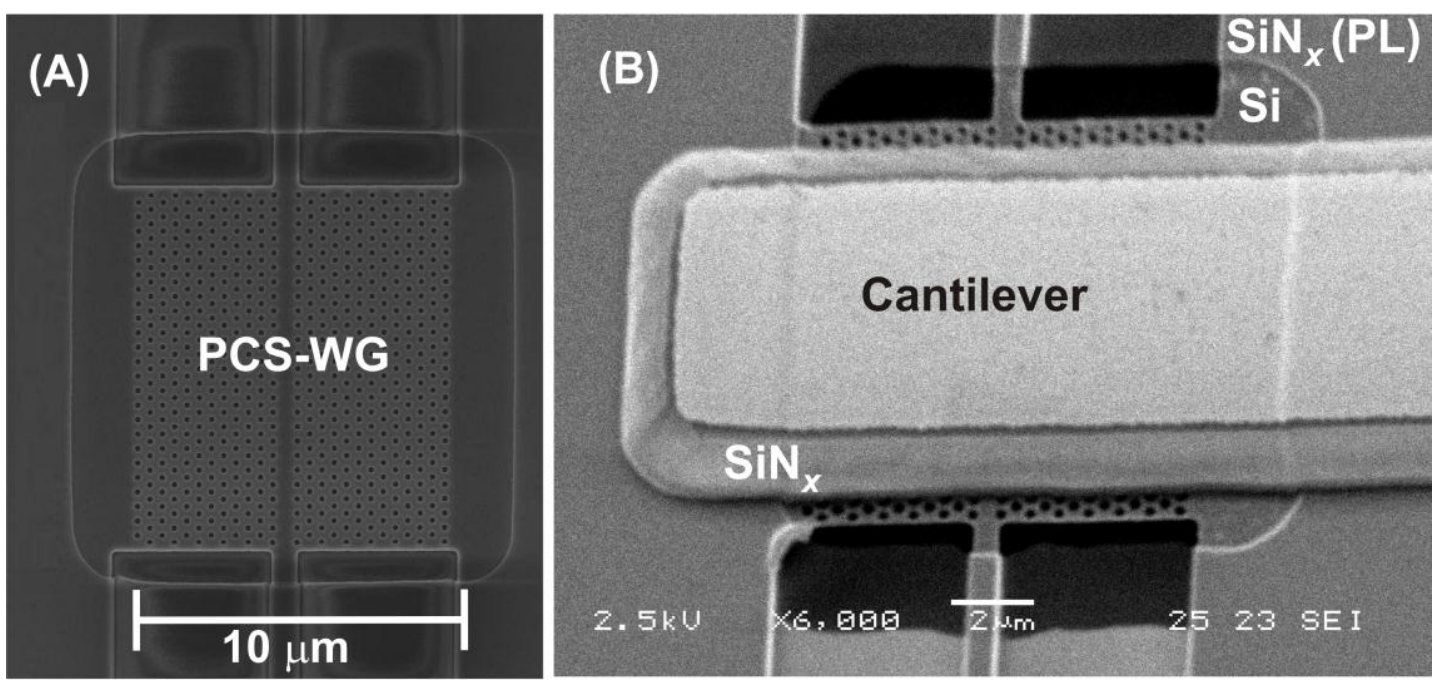

Figure 6.11. (A) Top view of a PCS-WG after the protective nitride bas been removed from the PC area and (B) oblique view of the integrated bimorph cantilever.

\section{$>$ Bimorph cantilever}

Removal of the protective $\mathrm{SiN}_{x}$ from the PC area leaves the conformal deposition of the bimorph layer with a step at the edges of the etch window (Figure 6.12). Due to this and by the upward deflection of the bimorph, the vertical gap between the PCS-WG and the bimorph varies along the length of the bimorph. 
Chapter 6. Photonic crystal slabs with integrated micro-cantilevers

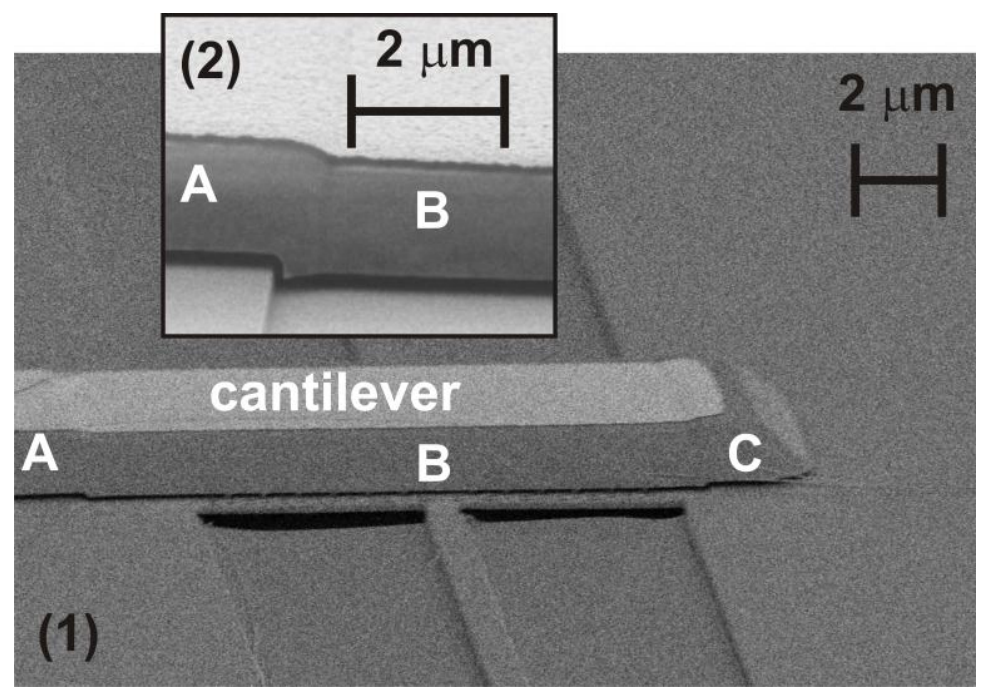

Figure 6.12. SEM images showing the different sections of the bimorph with varying vertical air-gaps. The inset shows a zoom-in of the step in the cantilever.

As shown in Figure 6.12, the gap consists of three sections namely A, B and C. The gap outside the etch window (part $\mathrm{A}$ and $\mathrm{C}$ ) is the combined thickness of the PL, the remaining $\mathrm{SiO}_{2}$ (TEOS) etch-mask layer, the SL and the off-state deflection. But the gap on top of the PC area (part B) is only due to the thickness of the SL and the off-state deflection of the bimorph.

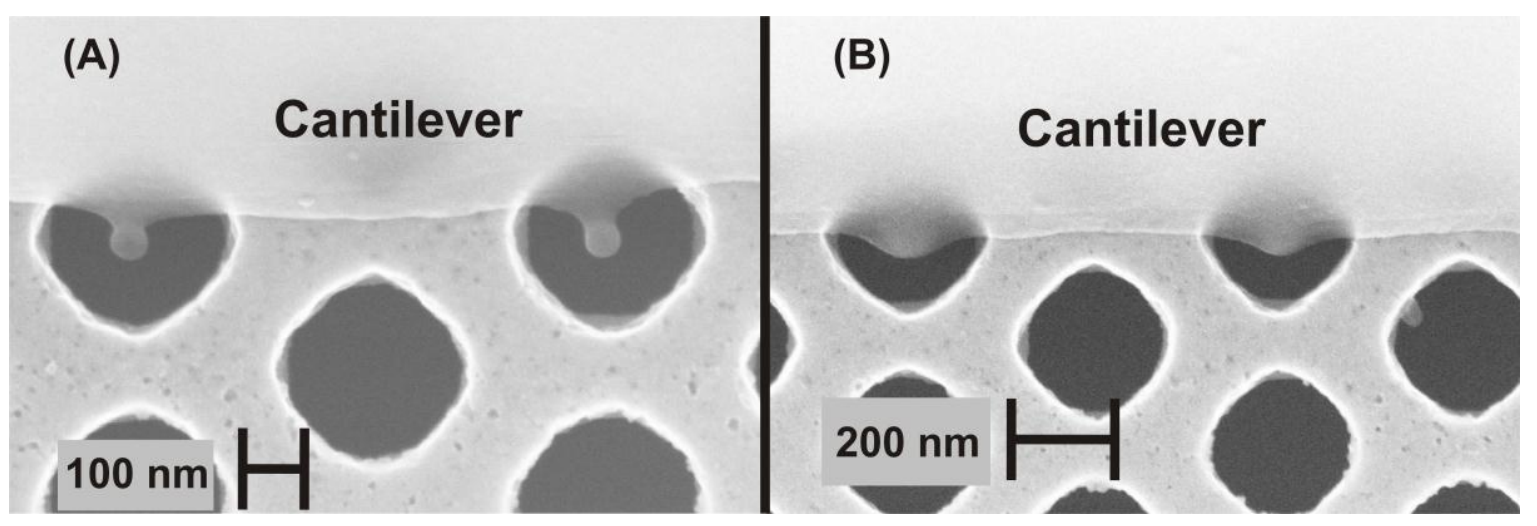

Figure 6.13. Top view showing (A) the tips on the sides of the cantilever and (B) tips removed by the photoresist retraction.

Due to the retraction of the photoresist during plasma etching (see section 4.3.4), the integrated cantilevers do not have the perfect rectangular geometry, as designed. Instead, the sides of the cantilever are tapered as shown in Figure 6.11 B. This also had an effect on the self-aligned tips on the sides of the cantilever. For some integrated devices, selfaligned tips were seen on the sides of the cantilever edges (Figure 6.13A) while for others the tips were absent due to the retraction of the photoresist (Figure 6.13B). 


\subsubsection{Photochemical etching of silicon in BHF}

Photochemical etching of silicon in aqueous HF [18] is an undesired mechanism in our process as it makes the silicon porous. In order to avoid this SLE was done in a dark environment. Figure 6.14A shows a collapsed photonic crystal (without cantilever) when the etching was not done in a dark environment. Figure 6.14B, C shows a PCS-WG, with integrated bimorph, for which the hole diameter is increased by this mechanism. As the PCS-WG properties are strongly dependent on the hole diameter even a few nanometres of undesired photochemical silicon etching can seriously damage the device.

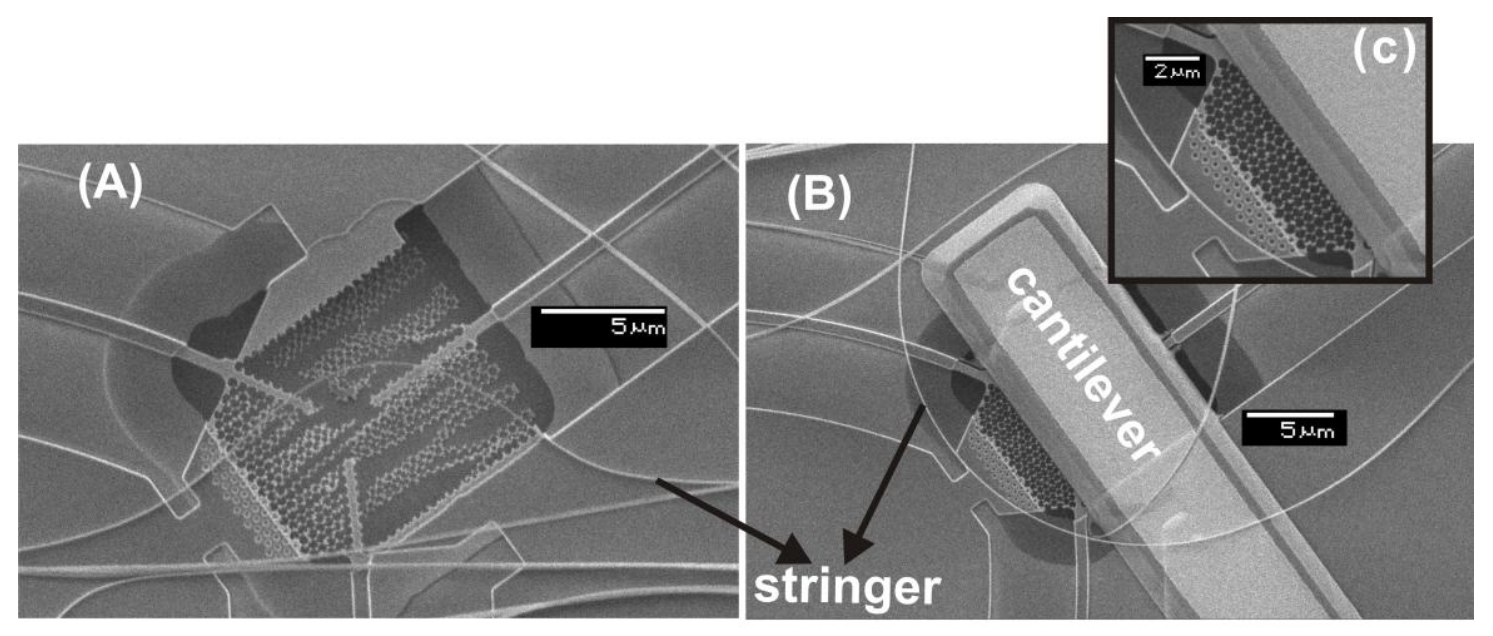

Figure 6.14. Photochemical etching of silicon in BHF showing $(A)$ a PC collapsed into the handle wafer and (B) with magnified inset (C), undesired increase of the PC bole diameter.

\subsection{Mechanical characterisation}

In order to assess its performance, the integrated device is mechanically characterized both statically and dynamically. The off-state tip deflection and the pull-in voltages of the bimorphs are measured at atmospheric pressure by the White Light Interferometer (WLIM) feature of a Polytec MSA400. Off-state deflection is the distance between the tip and the base of the bimorph (Figure 6.1C) and it is $20 \mathrm{~nm}$ for a bimorph of length $40 \mu \mathrm{m}$. 
Chapter 6. Photonic crystal slabs with integrated micro-cantilevers

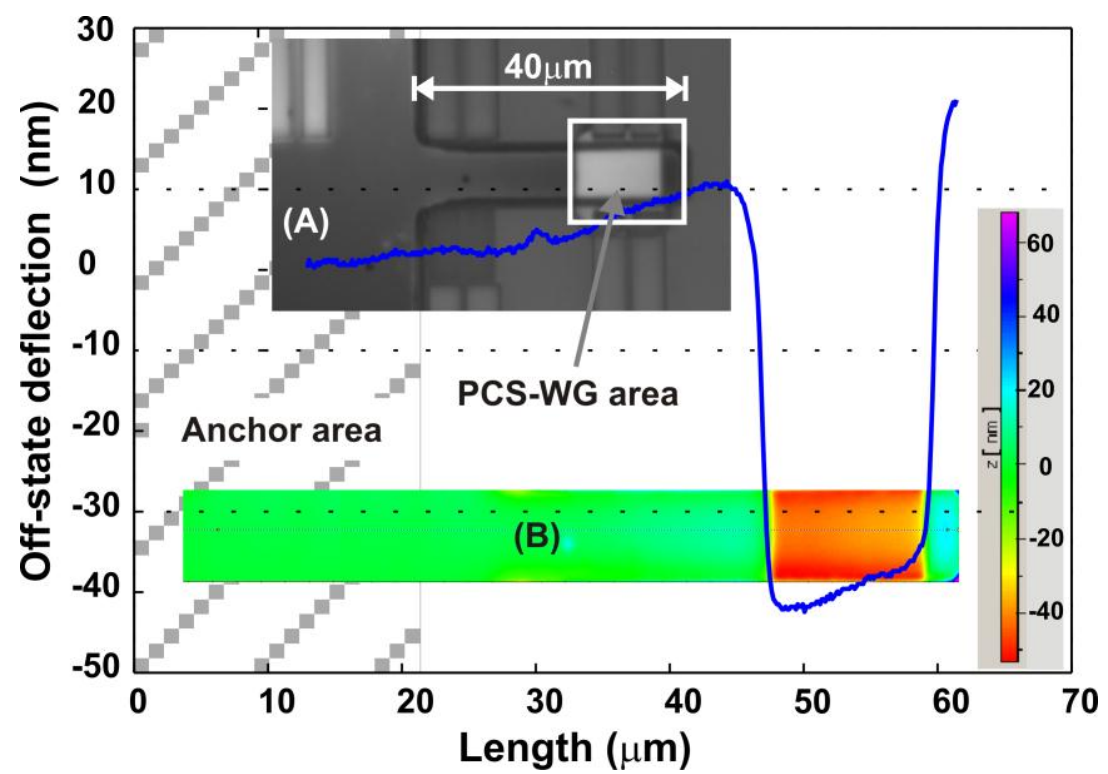

Figure 6.15. Static measurements on the off-state tip deflection of the bimorph (A) showing the optical microscopic image of the integrated device and (B) showing the 2-D surface map of the cantilever measured by WLIM.

Figure 6.15A shows an optical microscopic image of an integrated device using interference contrast. Along the length of the beam and above the PC area there is a downward step due to the etching of the $43 \mathrm{~nm}$ thick PL. This part is seen as a bright area in Figure 6.15A. Figure 6.15B shows a 2-D surface map of the cantilever by WLIM also demonstrating the downward stepping of the beam in the PC area.

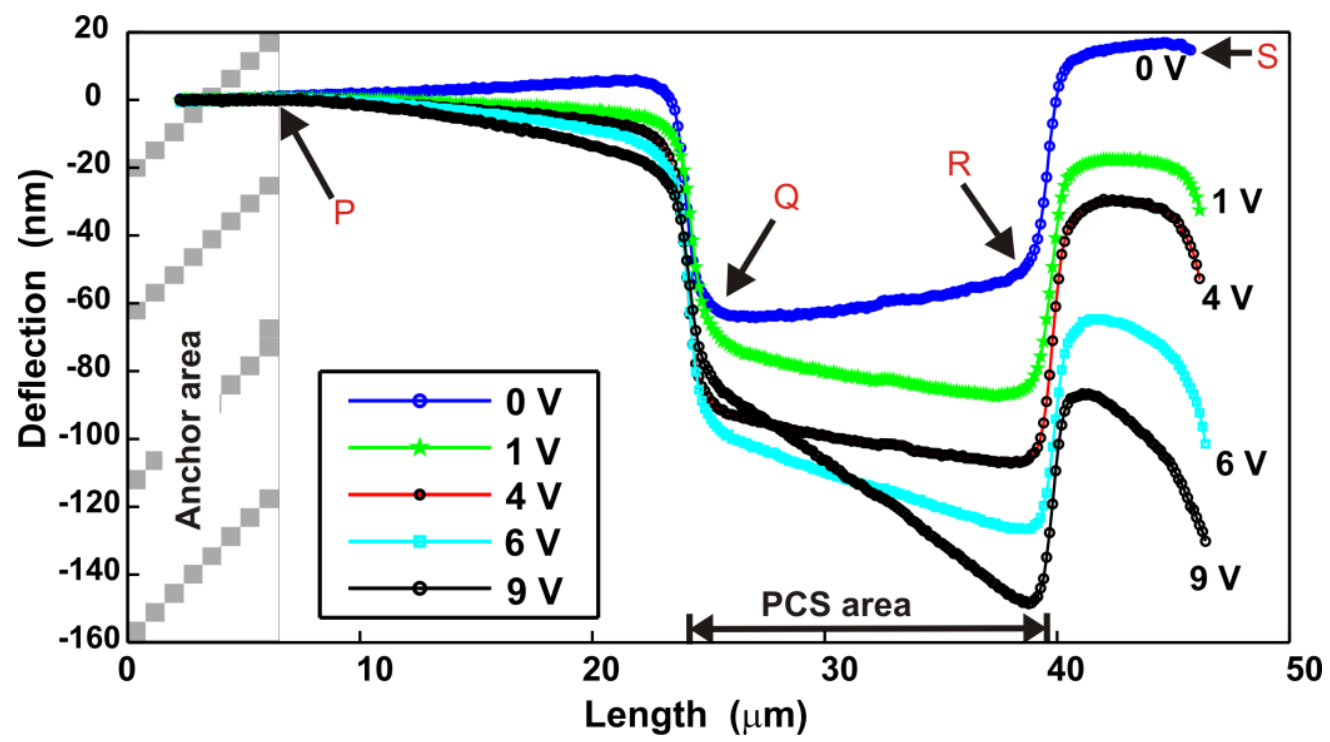

Figure 6.16. Static bimorph shapes for various voltages up to the point where the cantilever has touched the PC area at 9V.

The deformation of the shape of the bimorph on application of an increasing voltage is measured by the WLIM (Figure 6.16). In the off-state, the tip of the beam (point $S$ in 
Figure 6.16) has an upward displacement of $17 \mathrm{~nm}$ compared to the base of the beam (point P). On application of the voltage it advances towards the $\mathrm{PC}$ and at $9 \mathrm{~V}$, point $\mathrm{R}$ of the beam touches the PC slab membrane. At this voltage it also seems that the beam hinges around point $\mathrm{R}$ such that point $\mathrm{Q}$ at $9 \mathrm{~V}$ is higher than at $6 \mathrm{~V}$.

Dynamic measurements by Laser Doppler Vibrometery (LDV, Polytec MSA400) are carried out under controlled pressure, to access damping effects. The first mode resonance frequency of a $40 \mu \mathrm{m}$ long bimorph is at $821 \mathrm{kHz}$ with a quality factor of 591.5 at 1 mbar pressure (Figure 6.17). At atmospheric pressure the device is near-critically damped making it to operate faster than when it is either over- or under-damped (Figure 5.9).

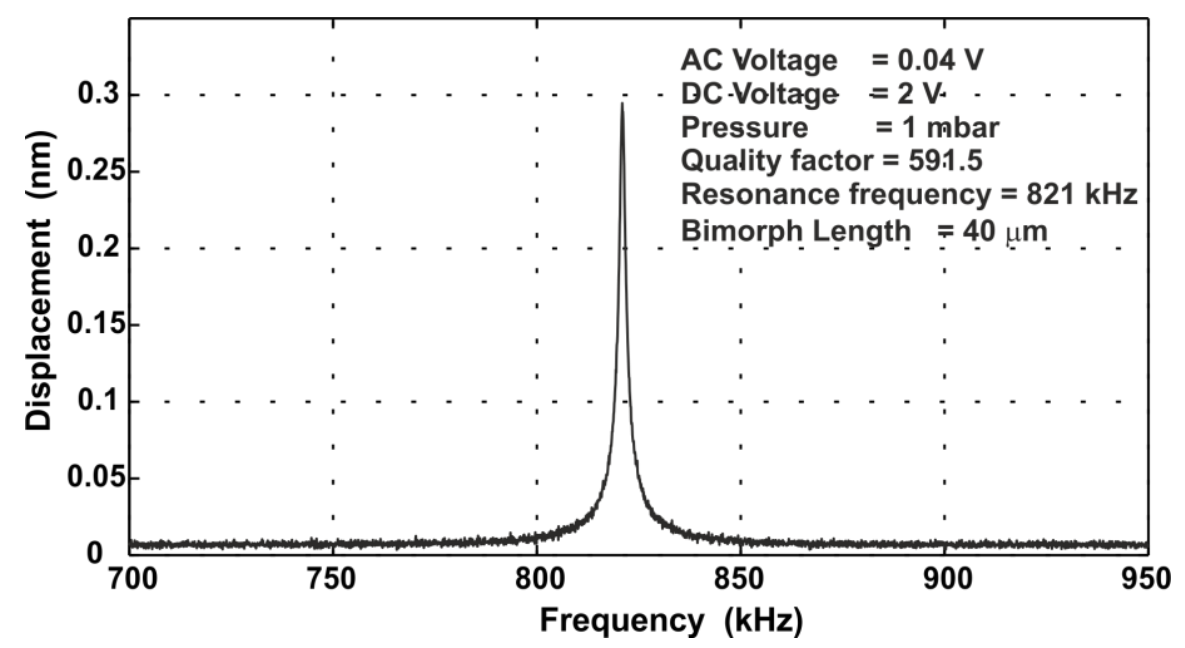

Figure 6.17. Dynamic measurement on the first mode resonance frequency of the bimorph.

\subsection{Optical characterisation}

Optical characterisation is carried out using the set-up described in section 5.3.1. Infrared light ( $1 \mathrm{~mW}$ at $1530 \mathrm{~nm}$ wavelength) from a tunable laser is coupled through a polarization-maintaining fibre (PMF) and an integrated grating coupler [19-20] into the optical device. Output light is recollected from an output grating coupler and is guided to a photodetector through a single-mode fibre (SMF). Figure 6.18 shows a 3-D schematic of the integrated device with its vertical fiber couplers and electrical contact probes. In order to avoid electrical insulation by native oxide formation on the silicon handle wafer, the wafer is silver glued to a copper plate, onto which the lower electrical contact is made. 


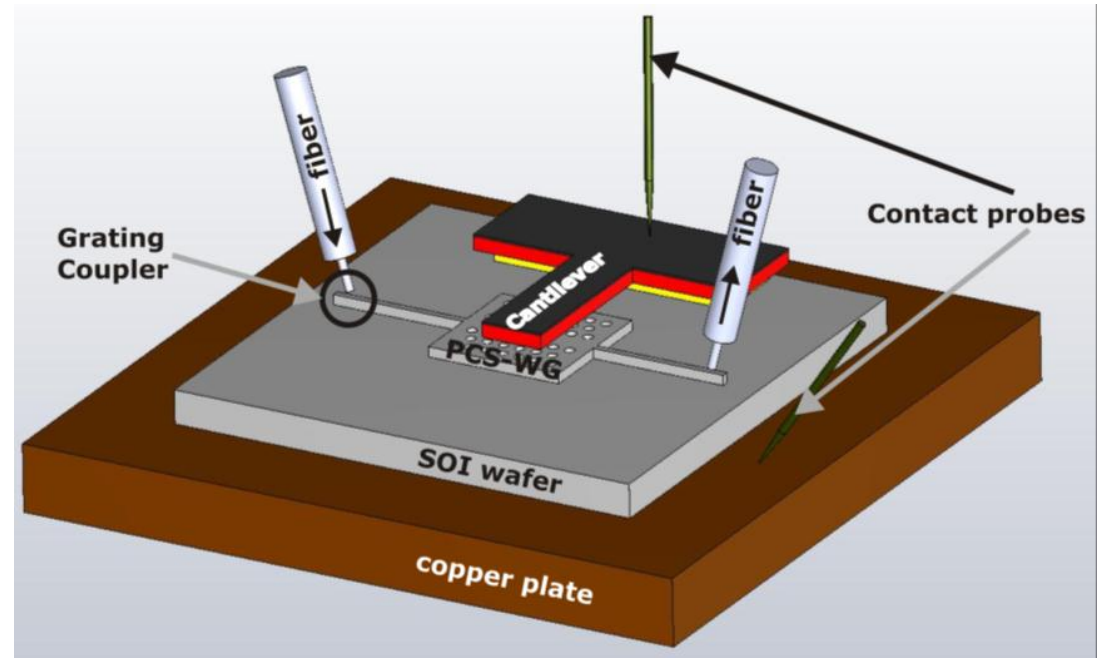

Figure 6.18. Schematic 3-D illustration of the integrated device with vertical fiber couplers and electrical contact probes (Image source: E. Berenschot, TST Group, University of Twente, The Netherlands).

We have observed about $80 \%$ throughput modulation of the integrated device, for interaction lengths as short as $10 \mu \mathrm{m}$, by applying a square wave drive signal of 0 to $8 \mathrm{~V}$ at $1 \mathrm{kHz}$ (Figure 6.19). The rise time of the switched optical signal is $85 \mu$ s and the fall time is $95 \mu \mathrm{s}$. As evidenced by the time response of the bimorph (Figure 5.10), measured by the LDV showing rise and fall times of $2 \mu$ s each, the increased time delay in the optical modulation measurement is not inherent to the device but, as determined independently, introduced by the large rise-time of the detector.
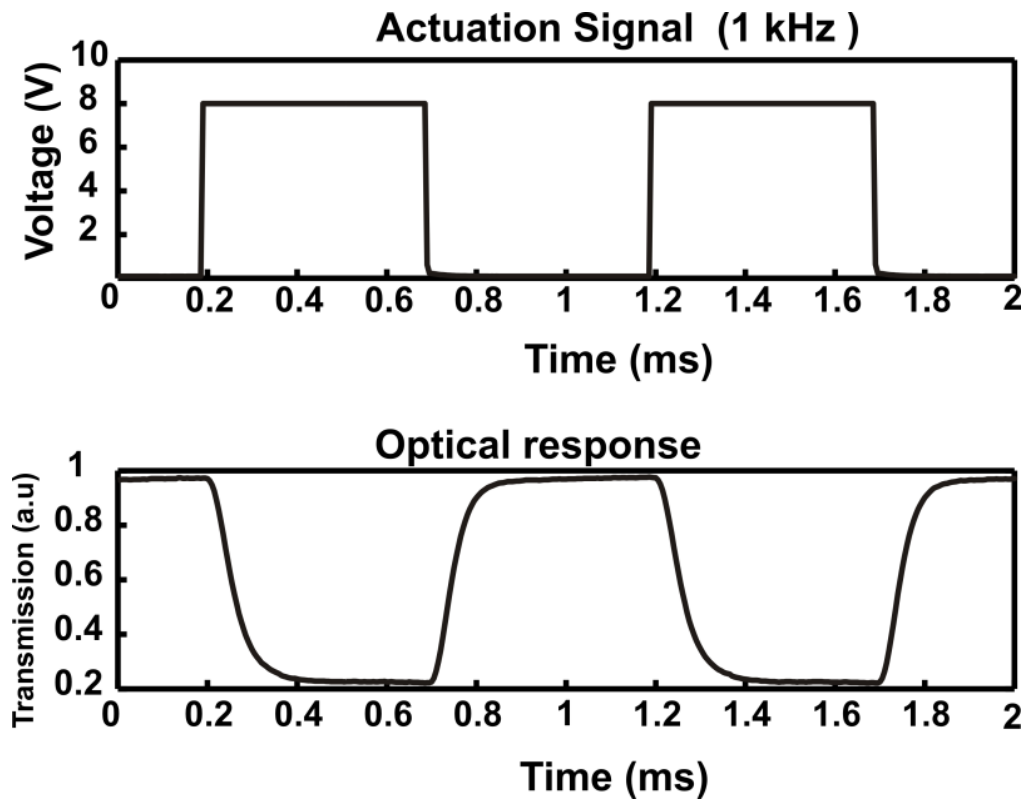

Figure 6.19. Optical modulation at $1 \mathrm{kHz}$ with a square wave drive signal of $0-8 \mathrm{~V}$.

The exact reason why the propagation loss increases when the cantilever is actuated closer to the PC is not known. Mode cut-off of the PCS-WG can be excluded as a potential mechanism, as it occurs at a wavelength longer than $1620 \mathrm{~nm}$ for all cantilever positions. 
As the cantilever moves closer to the waveguide, the cut-off wavelength is moved further away from the operating wavelength. There are two potential reasons: the dispersion properties change such that a part of the light is diffracted from the PC slab waveguide (light line crossing), or the presence of the cantilever close to the PC slab introduces such an abrupt index change that it leads to strong out-of plane scattering.

\subsection{Conclusions}

A process that enables the fabrication of electrostatically driven bimorph cantilevers with tips that are self-aligned with respect to the holes of a photonic crystal slab waveguide is demonstrated. This wafer-scale technology allows for the fabrication of a new class of devices exploiting modulation of the waveguiding properties of PCS-WGs by changing the proximity of the tips to (and into) the holes of the PC. As an example a MEMS integrated PCS-WG modulator has been fabricated. Optical modulation of the device at 1 $\mathrm{kHz}$ with a square wave drive signal of $0-8 \mathrm{~V}$ has been demonstrated with $80 \%$ modulation of the throughput in a waveguide section as short as $10 \mu \mathrm{m}$. 
Chapter 6. Photonic crystal slabs with integrated micro-cantilevers

\section{Part B: Micro-cantilever integrated 2-D photonic crystal microcavity based device for wavelength tuning}

A monolithically integrated micro-bimorph cantilever, equipped with tips that are self-aligned with respect to the holes of a 2-D photonic crystal microcavity based device (PCS-MC) is presented. On electrostatic actuation, the tips move into the holes and provide electromechano-optical modulation of light. The technology allows fabrication of tips with respect to specific photonic crystal holes by controlling the hole diameter and the sacrificial layer thickness. The integrated device is both mechanically and optically characterised. A $560 \mathrm{pm}$ wavelength shift and a maximum modulation of $21 \mathrm{~dB}$ is measured at the band edge (as measured from one of the output ports) of the PCS-MC, on application of a DC voltage as low as $0.75 \mathrm{~V}$.

\subsection{Introduction}

Channel-drop filters are essential components of the future photonic circuits. Among the various proposed designs, photonic crystal cavity based channel-drop filters [21] are attractive owing to their compact size and unique properties associated with photonic crystals. However to realise an active device, it is important to be able to tune the photonic crystals or photonic crystal slab dispersion properties. Tuning in photonic crystals can be realised by various methods such as thermo-optically [2], electro-optically [22], with liquid crystal infiltration [23] etc (for details see section 2.1). Apart from this, evanescent field perturbation using external mechanical probing has been established as an effective way to change the localised field inside a cavity. This has been demonstrated using tips of an atomic force microscope (AFM) [8-9, 24], fibre/glass [25-26] and by scanning near field optical microscope (SNOM) [27-28]. However, due to the lack of monolithic integration and the difficulty in aligning more than a single or a few tips in one device, this method becomes impractical.

Compared to the aforementioned methods, an electrostatically actuated integrated micro-cantilever has distinct advantages such as higher switching speed, compactness of the device and near-zero energy dissipation in the stationary state. Recently, an integrated electrostatically actuated comb drive was used to mechanically tune a 1-D PC nano-cavity using the coupled cavity effect [29-30] where the tuning is accomplished by varying the gap between two nano-cavities. Here, we demonstrate monolithic integration of a microcantilever, equipped with tips that are self-aligned with respect to the holes of a SOI based, with a 2-D air-bridge type photonic crystal microcavity based device. The electrostatically actuated micro-cantilever provides electromechano-optical modulation of 
light by insertion of the tips into the PC holes. The mechanical characterisation of the integrated device is reported and optical tuning near the photonic band edges is demonstrated.

\subsection{Fabrication}

PCS-MCs [31-32] and their access waveguides are fabricated on SOITEC SOI wafers by the silicon photonics platform ePIXfab [13]. The device is designed to operate in the C-band $(1530-1565 \mathrm{~nm})$ of the telecommunication wavelengths. Figure 6.20A shows a schematic of a single cavity based device. It consists of a line-type cavity (a nineholes-long section of a channel waveguide), evanescently coupled to two symmetrically arranged line-defect channel waveguides at a distance of three rows of holes. All waveguide sections are of a modified W1 type along the $\Gamma \mathrm{K}$-direction: one row of holes is omitted from the PC slab, and the diameters of the boundary holes have been modified in order to fine-tune the optical properties [17]. For an explanation of the operating principle [21] and a detailed design of the device we refer to [17]. Drop wavelength tuning can be achieved by perturbing the PCS-MC with an integrated micro-cantilever as shown in Figure 6.20B (top view) and Figure 6.21 (cross-sectional views in $x$ and $y$ directions).

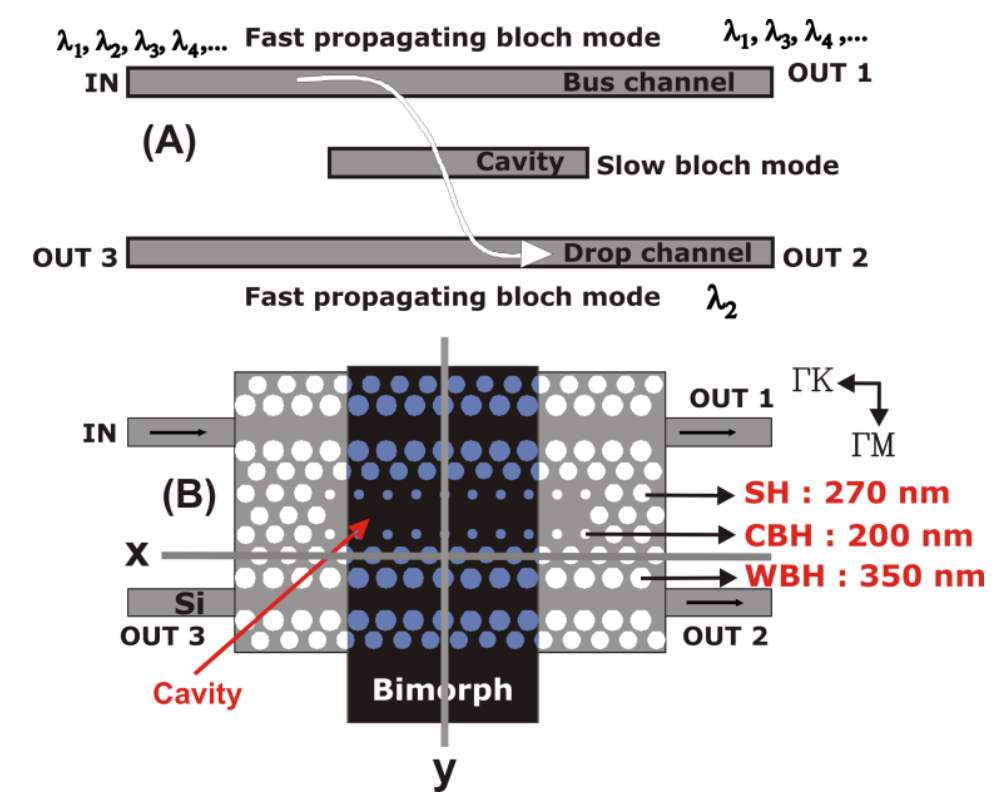

Figure 6.20. Schematic illustrating (A) cavity based wavelength selective channel-drop filter and (B) top view of the micro-cantilever integrated PCS-MC. 


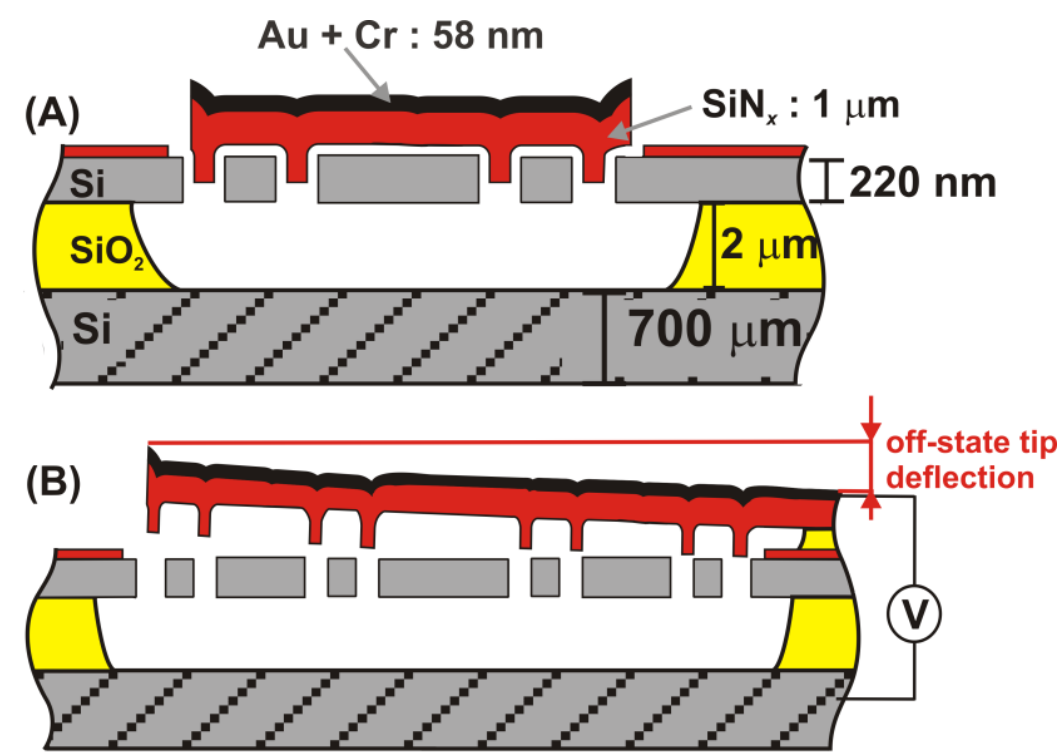

Figure 6.21. Schematic showing (A) cross-sectional view through the slab holes in the $x$-direction and (B) cross-sectional view through the $y$-direction.

The lattice constant of the structure is designed to be $440 \mathrm{~nm}$ and the nonboundary holes further referred to as slab holes $(\mathrm{SH})$, have a diameter of $270 \mathrm{~nm}$. The diameter of the $\mathrm{W} 1$ waveguide boundary holes $(\mathrm{WBH})$ is $350 \mathrm{~nm}$ and that of the cavity boundary holes $(\mathrm{CBH})$ is $200 \mathrm{~nm}$. All holes are air-filled. The thickness of the silicon device layer (i.e. the PC slab thickness) is $220 \mathrm{~nm}$ and that of the handle wafer is $700 \mu \mathrm{m}$. Before integration, the lower cladding is a $2 \mu \mathrm{m}$ thick thermal oxide (refractive index $n=$ 1.449 measured at $1550 \mathrm{~nm})$ and the upper cladding is air $(n=1.0)$. All claddings of the final integrated device are air. Figure 6.22A shows an SEM image of the PCS-MC with its access waveguides whereas Figure $6.22 \mathrm{~B}$ shows a detailed view of the cavity area indicating different hole diameters. The access waveguides of the PCS-MC are designed to be single-mode for TE-polarised light at a wavelength of $1550 \mathrm{~nm}$. 


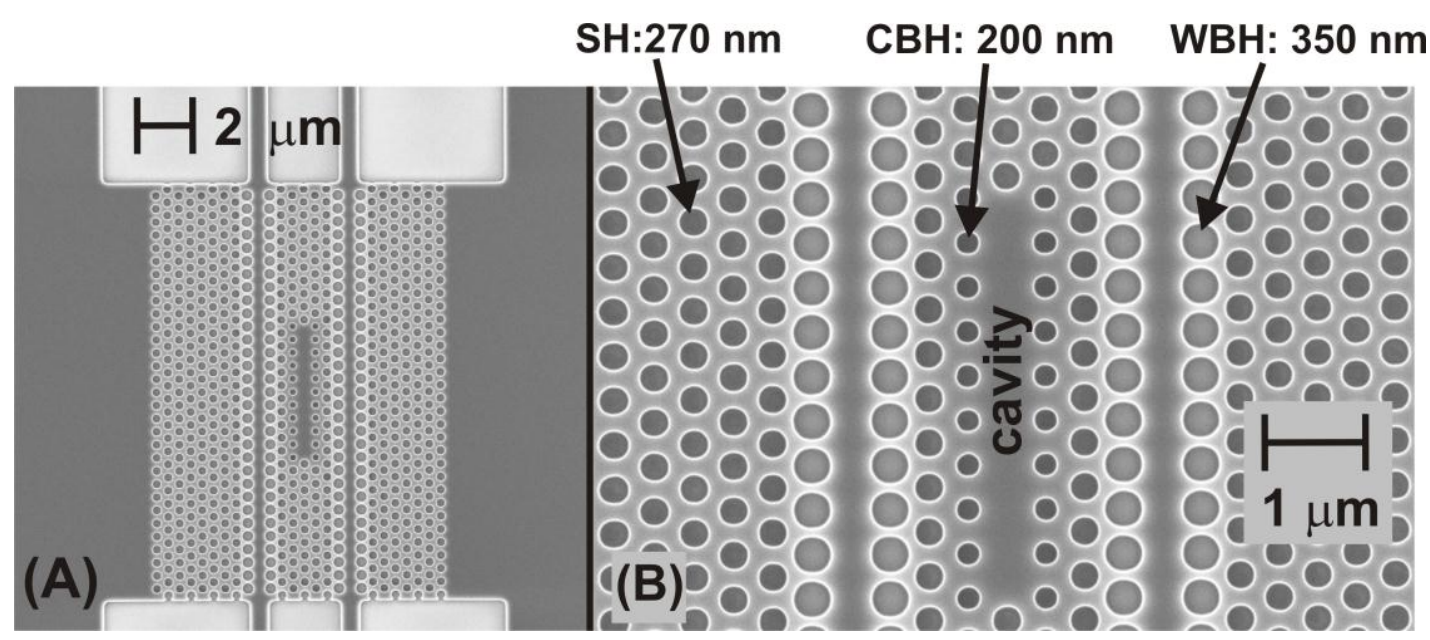

Figure 6.22. SEM images showing top views of $(A)$ a PCS-MC with its access waveguides and (B) a detailed view of its cavity area.

The technology for integration of a micro-cantilever with a PCS-MC is identical to the technology discussed in section 6.2. Figure 6.23 shows SEM images taken at different angles with respect to the integrated device whereas Figure 6.24 shows zoom-ins of the self-aligned tips.
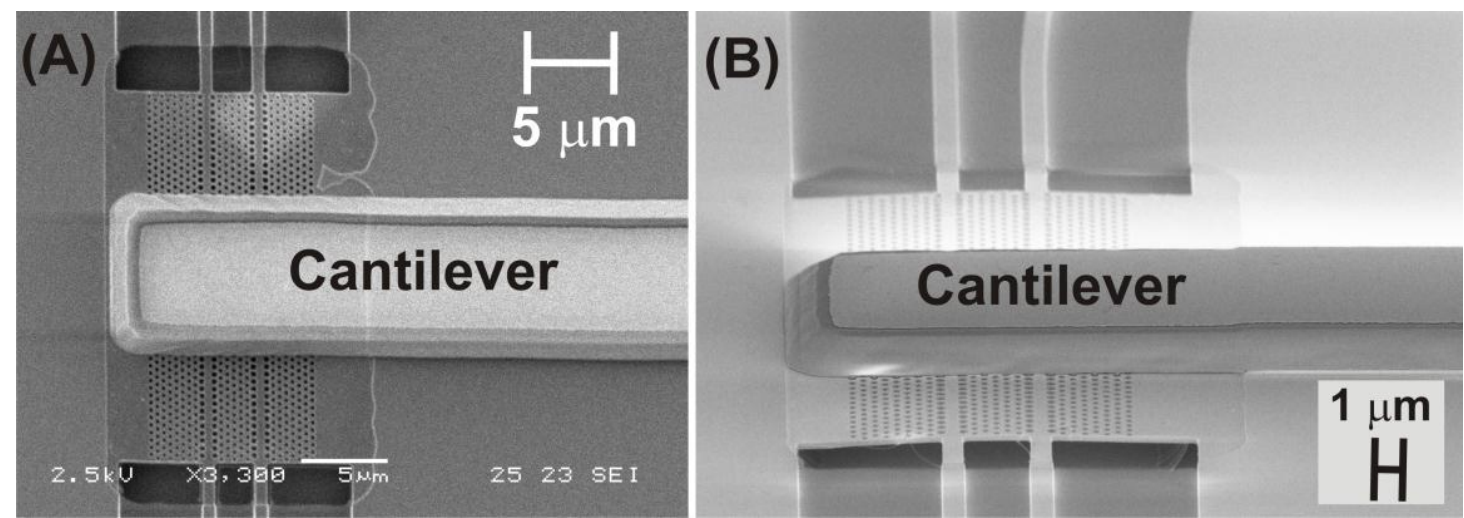

Figure 6.23. SEM images of (A) top view and (B) oblique view of the integrated device.

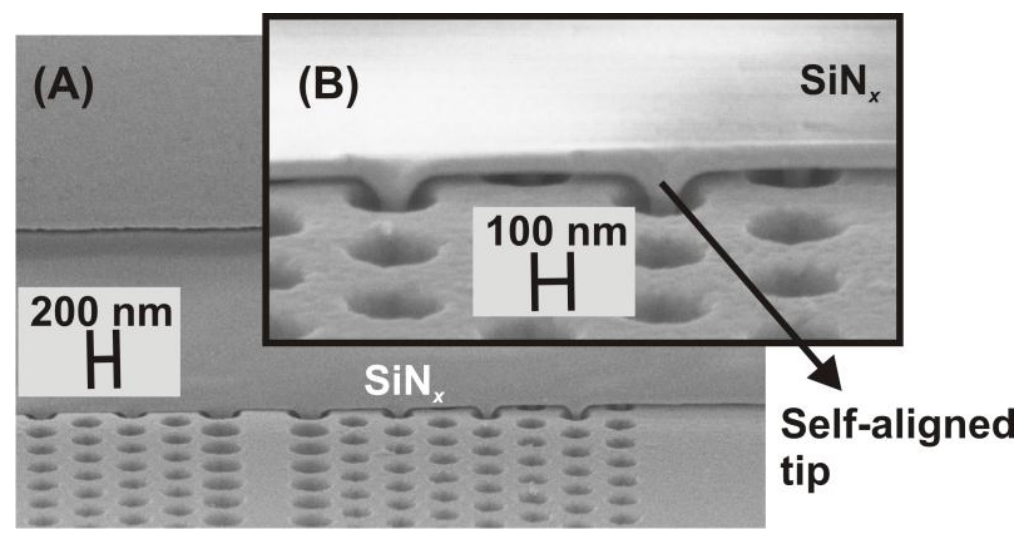

Figure 6.24. SEM images showing the self-aligned tips (A) on top of SH's and WBH's and (B) a zoom-in on top of two SH's. 
The height and width of the fabricated tips are strongly related to the thickness of the SL $\left(t_{\mathrm{SL}}\right)$ and the hole diameter as discussed in section 6.3.2. Figure 6.25 shows the schematic tip formation in three different holes for an increased sacrificial layer thickness. Until $t_{\mathrm{SL}}$ is $100 \mathrm{~nm}$ (half of the smallest PC hole diameter), all three holes will have a self-aligned tip of $220 \mathrm{~nm}$ height on top. As soon as the thickness increases above $100 \mathrm{~nm}$, the tip on top of the $\mathrm{CBH}$ will reduce its height and will convert to a sharp pin. Similarly until $t_{\mathrm{SL}}=135$ $\mathrm{nm}$, all the SHs will have $220 \mathrm{~nm}$ high self-aligned tips on top and until $t_{\mathrm{SL}}=175 \mathrm{~nm}$ all the WBHs will have $220 \mathrm{~nm}$ high tips on top. Since an acceptable yield of devices becomes increasingly challenging as $t_{\mathrm{SL}}$ decreases, we have selected SL to be $100 \mathrm{~nm}$ in this process. After deposition the measured SL thickness is $105 \mathrm{~nm}$. Moreover, the measured PC hole diameter ranges $\pm 20 \mathrm{~nm}$ around its designed value [17] as an exposure sweep is performed during the deep-UV lithography. Hence, with a $t_{\mathrm{SL}}=105 \mathrm{~nm}$, all the $\mathrm{SH}$ and WBH will have $220 \mathrm{~nm}$ high self-aligned tips on top. However the height of the tips on top of $\mathrm{CBH}$ will be considerably less than $220 \mathrm{~nm}$ given the hole diameter of 200 $\mathrm{nm}$. Figure 6.26 shows the SEM images of self-aligned tips on top of SH and WBH, where the PCS-MC has been removed locally from the wafer. The figure shows tips with a diameter of $\sim 60 \mathrm{~nm}(270-2 \times 105)$ arising from SH and $\sim 140 \mathrm{~nm}$ diameter $(350-2 \times$ 105) arising from WBH.

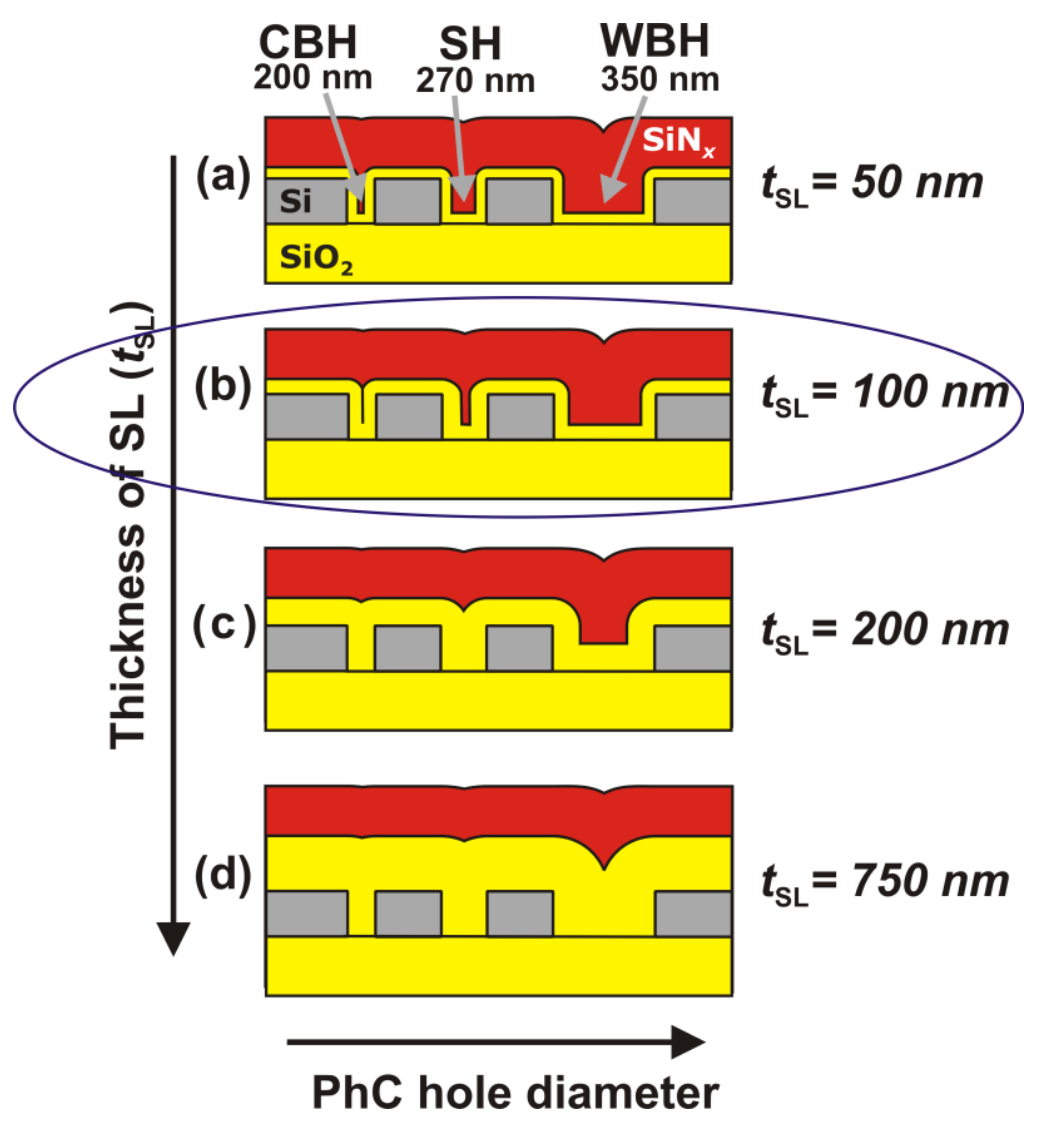

Figure 6.25. Effect of sacrificial layer thickness and hole diameter on the tip formation. 


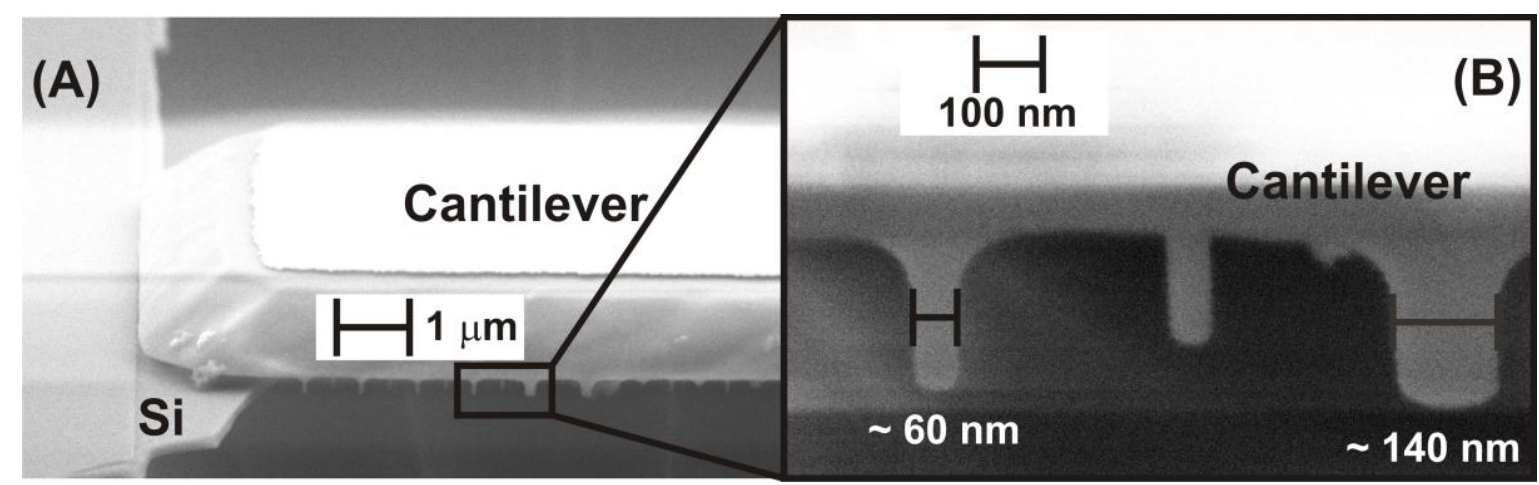

Figure 6.26. SEM images showing (A) self-aligned tips of different widths and (B) a zoom-in of two different types of tips where the PCS-MC has been locally removed.

\subsection{Mechanical characterisation}

In order to assess its performance the integrated cantilever is characterised statically. Offstate tip deflection, which arises due to the stress in the top electrode layer, is measured by white light interferometry (WLIM) showing $19 \mathrm{~nm}$ tip deflection for a $60 \mu \mathrm{m}$ long cantilever (Figure 6.27). Due to the local etching of the protective nitride layer in the PC area, the subsequently deposited conformal layers follow this step which gives the cantilever a stepped geometry on top of the PC as shown in Figure 6.23.

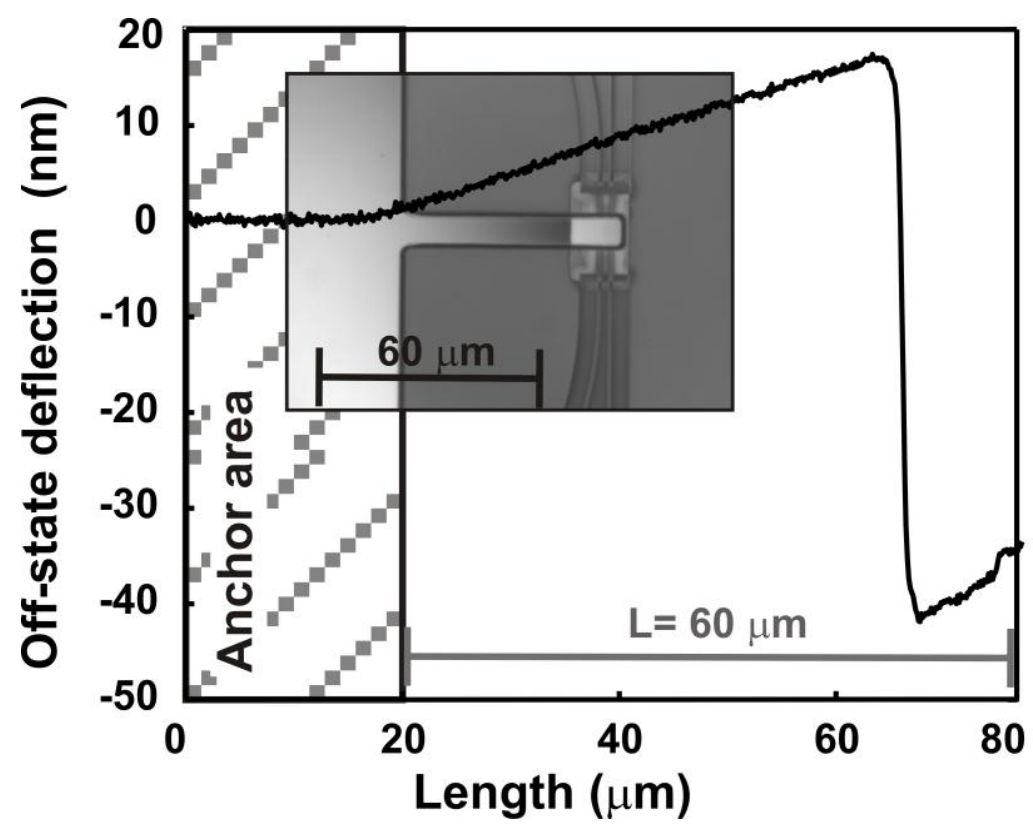

Figure 6.27. Plot of the off-state deflection of a $60 \mu \mathrm{m}$ long cantilever, measured by WLIM. The shaded region represents the anchor area of the cantilever. The inset shows an optical microscope image of the integrated device.

Static displacement of the bimorph on application of various DC voltages are measured and shown in Figure 6.28. 
Chapter 6. Photonic crystal slabs with integrated micro-cantilevers

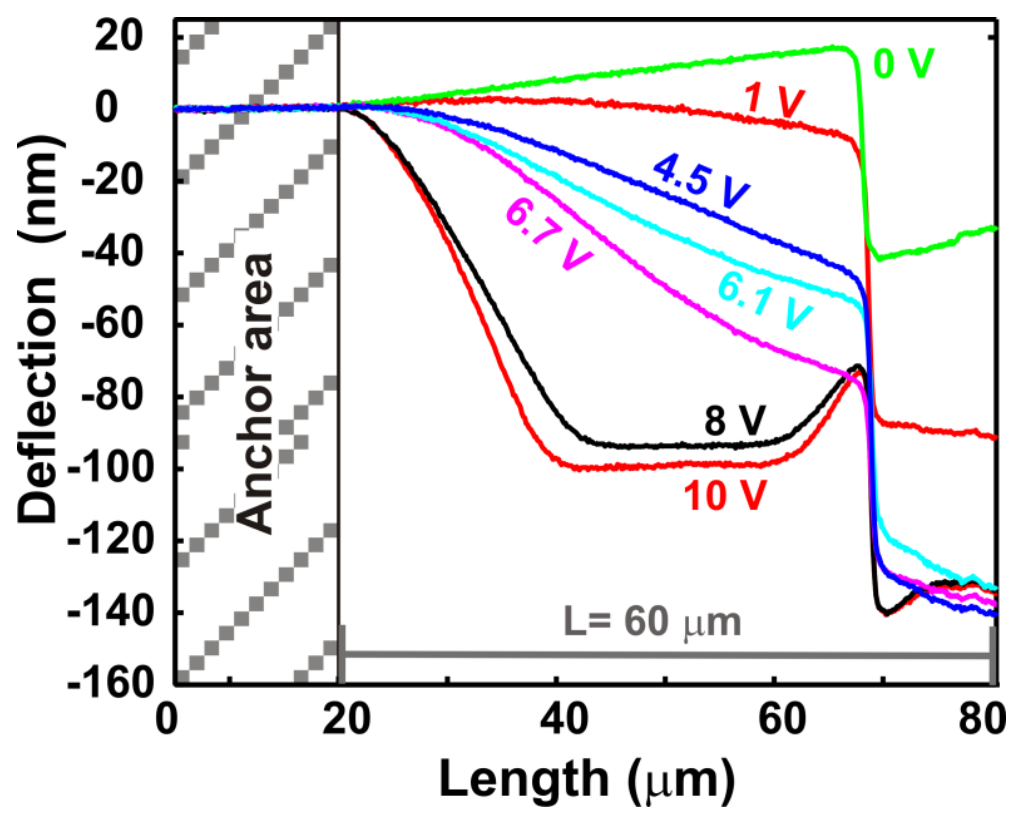

Figure 6.28. Static displacement of a $60 \mu \mathrm{m}$ long the cantilever on application of various DC voltages.

\subsection{Optical characterisation}

Optical characterisation is carried out using the set-up described in section 5.3.1. Infrared light $(500 \mu \mathrm{W})$ from a tunable laser (Agilent 8164B and 81634B) is coupled through a polarization-maintaining fibre and an integrated grating coupler $[17,20]$ into the optical device. Output light is recollected from an output grating coupler and is guided to the photodetector through a single-mode fibre. The silicon handle wafer of the device is silver-glued to a copper plate after removing the native oxide from its backside, to have a good electrical connection. Figure 6.29 shows the 3-D schematic of the integrated device with its electrical contact probes and vertical fibre couplers. 


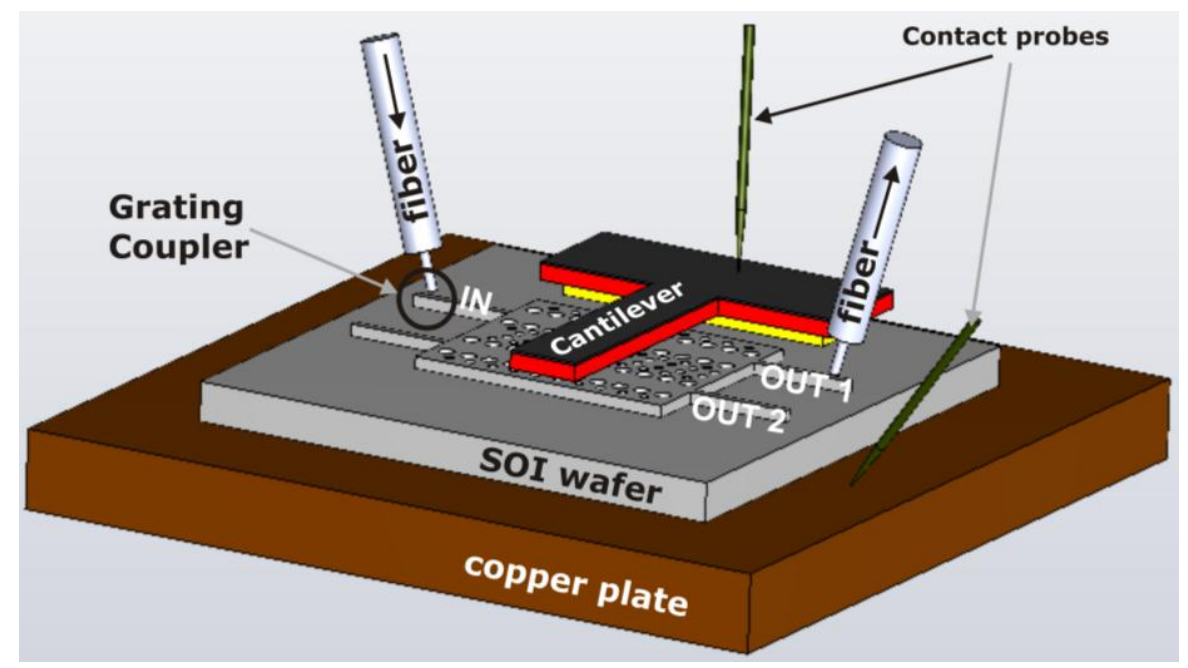

Figure 6.29. Schematic representation of the optical tuning measurement set-up with the vertical fibre couplers and electrical contact probes (Image source: E. Berenschot, TST Group, University of Twente, The Netherlands).

Figure 6.30 shows the measured transmission spectrum of the integrated device at the OUT 1 and OUT 2 ports when the cantilever is in its off-state. The measurement is carried out with a wavelength resolution of $10 \mathrm{pm}$. There is an indication of photonic band edges at $1496 \mathrm{~nm}$ and at $1604 \mathrm{~nm}$ with a $108 \mathrm{~nm}$ transmission-gap in between them. Since the line defect waveguides do not transmit light in these wavelengths-gap it may indicate that light can freely propagate through the photonic crystal structure. I.e. it would imply that the photonic crystal structures have (at least) two band-gaps.

At the third band edge, OUT 1 port shows a steep -35 dB decline in transmission whereas OUT 2 port shows a transmission reduction of $-20 \mathrm{~dB}$ (in the cantilever off-state). However according to the design, the third band gap should start at a wavelength of 1560 $\mathrm{nm}$ [17]. The discrepancy between the designed and the measured values might be attributed by the nanometre scale etching of silicon in $\mathrm{H}_{3} \mathrm{PO}_{4}$ (during protective $\mathrm{SiN}_{x}$ etching) or in BHF (during SLE). It is observed that the light is coupled to the OUT 2 port near the third band edge, possibly due to the presence of the cantilever. Moreover channel dropping at the resonant wavelength, which was intended in the design, is not observed. 


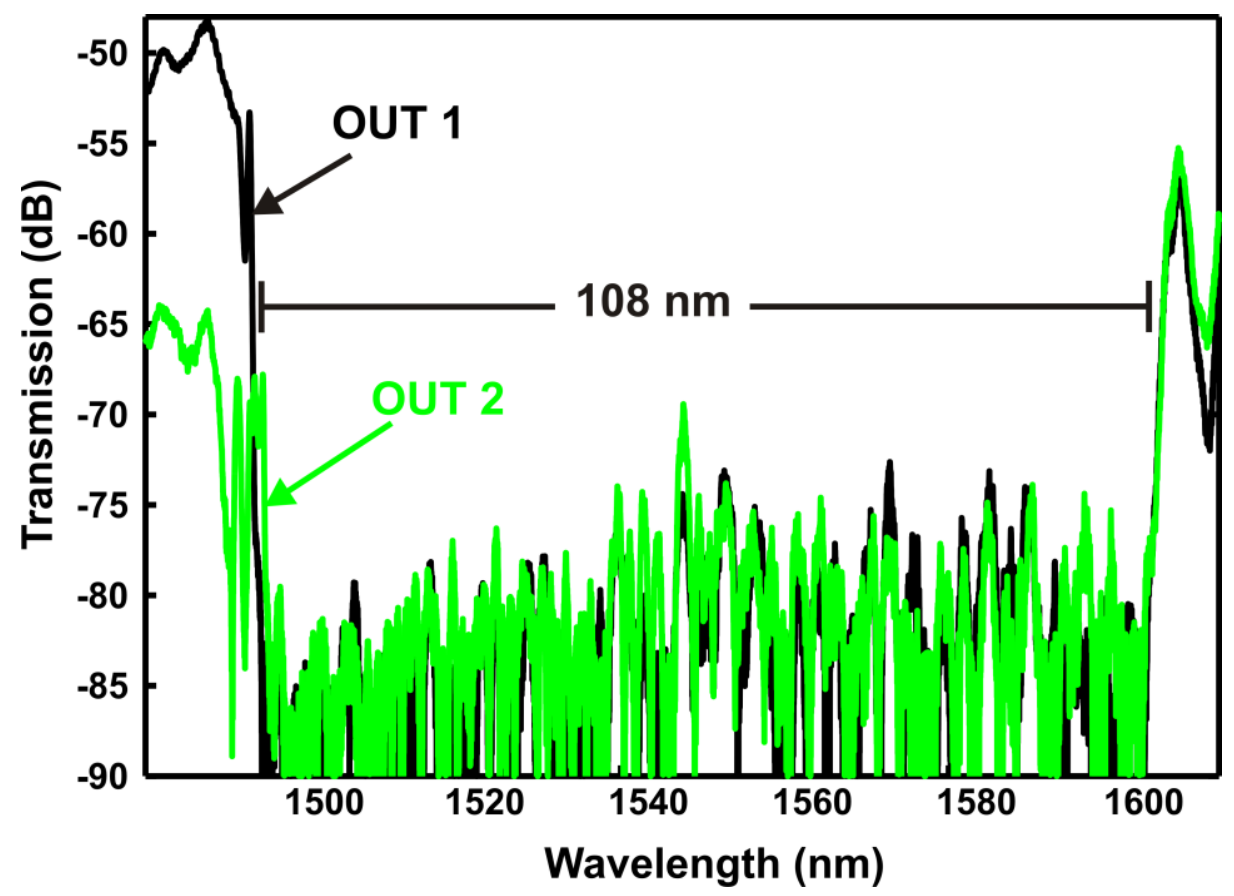

Figure 6.30. Transmission spectra of the PCS-MC in the OUT 1 and OUT 2 ports, when the cantilever is in its off-state $(0 \mathrm{~V})$.

However, wavelength tuning has been measured near the band edges by inserting the tips into the PC holes. A wavelength tuning of $560 \mathrm{pm}$ is observed at the third band edge of the OUT 1 port. A modulation as high as $21 \mathrm{~dB}$ is measured by applying a low DC voltage $(0.75 \mathrm{~V})$ to the cantilever (Figure $6.31 \mathrm{~A})$. Similarly wavelength tuning of $470 \mathrm{pm}$ is observed from the OUT 2 port at the third band edge at the same voltage. However, the measured modulation is only $12 \mathrm{~dB}$ (Figure 6.31B). Measurements were also carried out at the second band edge, demonstrating $\sim 500$ pm wavelength shift at the OUT 1 port and $\sim 460 \mathrm{pm}$ at the OUT 2 port. These tuning measurements were carried out with a resolution of $1 \mathrm{pm}$.
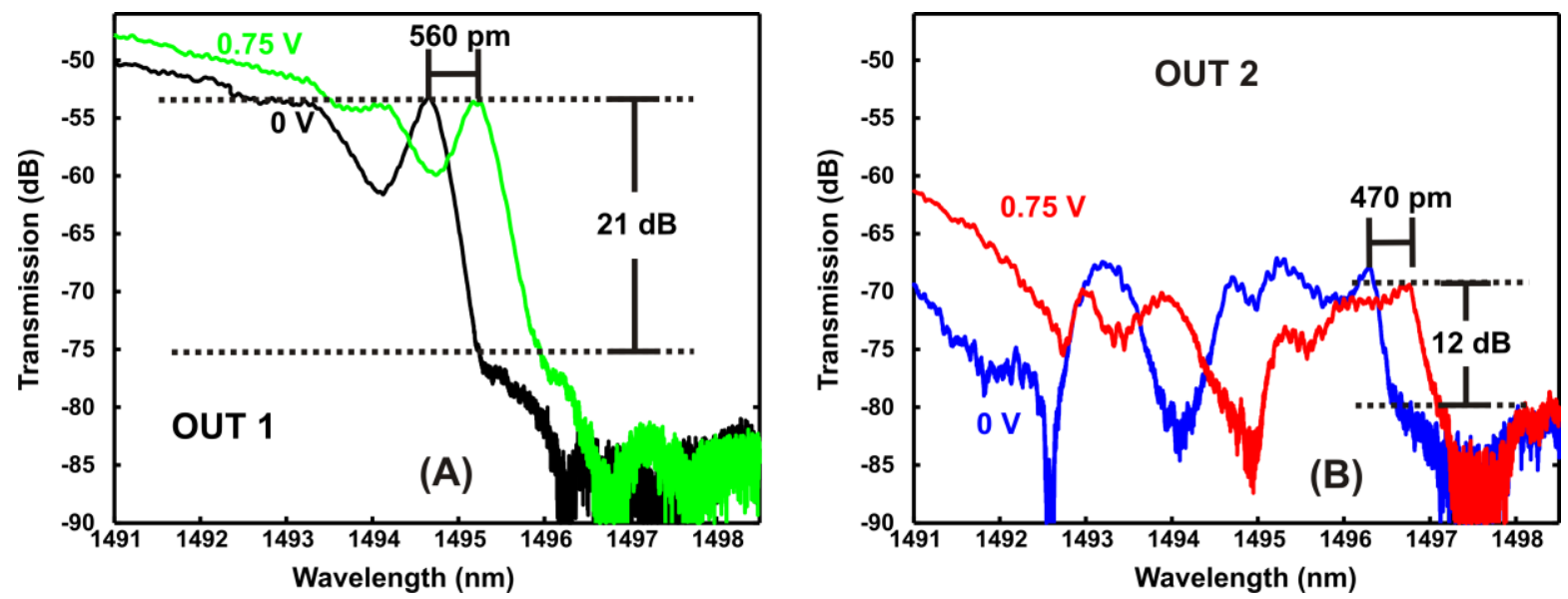

Figure 6.31. Static tuning of the third band edge of the PCS-MC in the (A) OUT 1 and (B) OUT 2 ports by applying a DC voltage of $0.75 \mathrm{~V}$. 

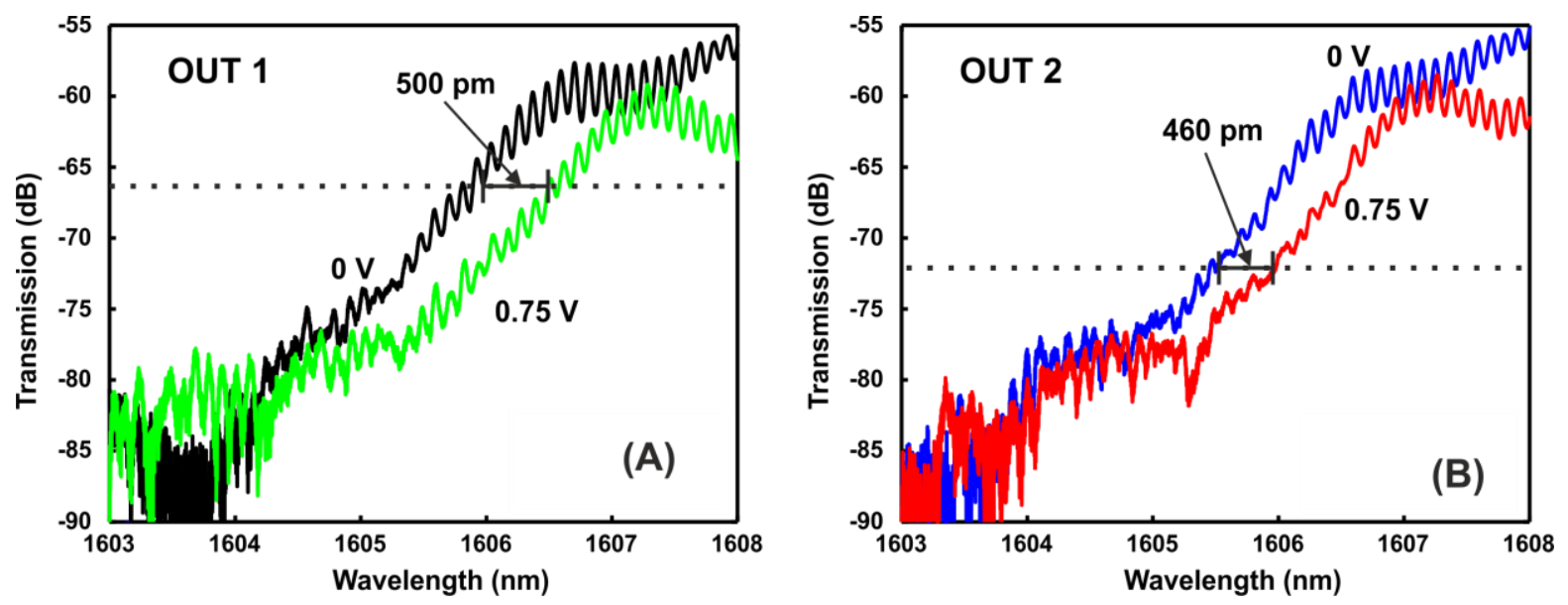

Figure 6.32. Static tuning of the second band edge of the PCS-MC in the (A) OUT 1 and (B) OUT 2 ports by applying a DC voltage of $0.75 \mathrm{~V}$.

\subsection{Conclusions}

A successful fabrication technology that enables the forming of electrostatically driven bimorph cantilevers with sharp tips that are self-aligned with respect to the holes of a photonic crystal microcavity based device is described. This CMOS-compatible technology allows for the fabrication of a new class of compact devices, exploiting modulation of the waveguiding properties of PCS-MCs by changing the proximity of the tips to (and into) the holes of the PC. As an example, a micro-cantilever integrated PCSMC modulator has been fabricated. The cantilever is mechano-optically characterised. It was shown that the third optical band edge, as measured from the OUT 1 port, could be statically tuned to a $560 \mathrm{pm}$ higher wavelength by a DC voltage as small as $0.75 \mathrm{~V}$.

\subsection{References}

[1] E. Yablonovitch, "Inhibited spontaneous emission in solid-state physics and electronics," Physical Review Letters, vol. 58, pp. 2059-2062, 1987.

[2] H. M. H. Chong and R. M. DeLaRue, "Tuning of photonic crystal waveguide microcavity by thermooptic effect," IEEE Photonic Technology Letters, vol. 16, pp. 1528-1530, 2004.

[3] M. Schmidt, et al., "Electro-optically tunable photonic crystals," Applied Physics Letters, vol. 87, pp. 121110-1-3, 2005.

[4] S. F. Mingaleev, et al., "Tunable photonic crystal circuits: concepts and designs based on single-pore infiltration," Optics Letters, vol. 29, pp. 2858-2860, 2004.

[5] W. Park and J. B. Lee, "Mechanically tunable photonic crystal structure," Applied Physics Letters, vol. 85, pp. 4845-4847, 2004.

[6] S. Iwamoto, et al., "Observation of micromechanically controlled tuning of photonic crystal line-defect waveguide," Applied Physics Letters, vol. 88, p. 011104, 2006.

[7] W. C. L. Hopman, "Light-flow characterization and manipulation in 1 and 2 dimensional photonic crystals," PhD Thesis, University of Twente, 2007.

[8] T. Takahata, et al., "Transmittance tuning of photonic crystal reflectors using an AFM cantilever," Sensors and Actuators A, vol. 128, pp. 197-201, 2006.

[9] I. Märki, et al., "Tuning the resonance of a photonic crystal microcavity with an AFM probe," Optics Express, vol. 14, pp. 2969-2978, 2006. 
Chapter 6. Photonic crystal slabs with integrated micro-cantilevers

[10] Y. Kanamori, et al., "Photonic crystal switch by inserting nano-crystal defects using MEMS actuator," in IEEE/LEOS Int.Conf.Optical MEMS, Piscataway,NJ, 2003, pp. 107108.

[11] W. Zhou, et al., "Novel reconfigurable semiconductor photonic crystal-MEMS device " Solid-State Electronics, vol. 50, pp. 908-913, 2006.

[12] T. Takahata, et al., "A wide wavelength range optical switch using a flexible photonic crystal waveguide and silicon rods," Journal of Micromechanics and Microengineering, vol. 20, pp. 075009-1-6, 2010.

[13] P. Dumon, et al., "Towards foundry approach for silicon photonics: silicon photonics platform ePIXfab," Electronics Letters, vol. 45, pp. 581-582, 2009.

[14] J. G. E. Gardeniers, et al., "LPCVD silicon-rich silicon nitride films for applications in micromechanics, studied with statistical experimental design," Journal of V acuum Science and Technology A: Vacuum, Surfaces and Films, vol. 14, pp. 2879-2892, 1996.

[15] R. Legtenberg and H. A. C. Tilmans, "Electrostatically driven vacuum-encapsulated polysilicon resonators Part I. Design and fabrication," Sensors and Actuators A, vol. 45, pp. 57-66, 1994.

[16] E. Sarajlic, et al., "Fabrication of 3d nanowire frames by conventional micromachining technology," in Proceedings of the 13th International Conference on Solid-State Sensors, Actuators and Microsystems, Seoul,Korea, 2005, pp. 27-30.

[17] L. J. Kauppinen, "Compact integrated optical devices for optical sensor and switching applications," PhD Thesis, University of Twente, 2010.

[18] K. W. Kolasinski, "The mechanism of Si etching in fluoride solutions," Physical chemistry chemical physics, vol. 5, pp. 1270-1278, 2003.

[19] L. J. Kauppinen, "Compact integrated optical devices for optical sensor and switching applications," PhD Thesis, University of Twente, 2010.

[20] D. Taillaert, et al., "Grating couplers for coupling between optical fibers and nanophotonic waveguides," The Japan Society of Applied Physics, vol. 45, pp. 6071-6077, 2006.

[21] S. Fan, et al., "Channel drop filters in photonic crystals," Optics Express, vol. 3, pp. 4-11, 1998.

[22] I. Fushman, et al., "Ultrafast nonlinear optical tuning of photonic crystal cavities," Applied Physics Letters, vol. 90, pp. 091118-1-3, 2007.

[23] B. Wang, et al., "Controlling mode degeneracy in a photonic crystal nanocavity with infiltrated liquid crystal," Optics Letters, vol. 35, pp. 2603-2605, 2010.

[24] W. C. L. Hopman, et al., "Nano-mechanical tuning and imaging of a photonic crystal micro-cavity resonance," Optics Express, vol. 14, pp. 8745-8752, 2006.

[25] A. F. Koenderink, et al., "Controlling the resonance of a photonic crystal microcavity by a near-field probe," Physical Review Letters, vol. 95, pp. 153904-1-4, 2005.

[26] F. Intonti, et al., "Spectral tuning and near-field imaging of photonic crystal microcavities," Physical Review B, vol. 78, pp. 041401-1-4, 2008.

[27] S. Mujumdar, "Tunability parameters in SNOM tip mediated tuning of an ultra-high quality heterostructure resonator " Journal of Optics, vol. 12, pp. 035001-6, 2010.

[28] L. Lalouat, et al., "Near-field interactions between a subwavelength tip and a smallvolume photonic-crystal nanocavity," Physical Review B, vol. 76, pp. 041102-1-4, 2007.

[29] X. Chew, et al., "Dynamic tuning of an optical resonator through MEMS-driven coupled photonic crystal nanocavities," Optics Letters, vol. 35, pp. 2517-2519, 2010.

[30] X. Chew, et al., "An in-plane nano-mechanics approach to achieve reversible resonance control of photonic crystal nanocavities," Optics Express, vol. 18, pp. 22232-22244, 2010.

[31] S. Fan, et al., "Theoretical analysis of channel drop tunneling processes," Physical Review B, vol. 59, pp. 15882-15892 1999.

[32] E. Drouard, et al., "Directional channel-drop filter based on a slow Bloch mode photonic crystal waveguide section," Optics Express, vol. 13, pp. 3037-3048, 2005. 


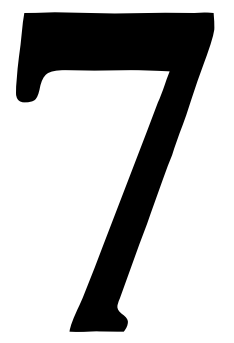

\section{Conclusions and outlook}

In this chapter a number of conclusions related to the results described in the thesis are drawn. These results relate to: modelling of the mechanical element, fabrication technology for monolithic integration and mechano-optical characterisation of the integrated optical devices. The chapter ends with an outlook on possible future research. 


\subsection{Conclusions}

Compact integrated mechano-optical wavelength selective devices based on three different types of photonic structures viz. a micro-ring resonator (MRR), a 2-D slab photonic crystal line-defect waveguide (PCS-WG) and a 2-D slab photonic crystal microcavity based device (PCS-MC) are depicted in this thesis. One of the focuses of the thesis is on the modelling and characterisation of the mechano-optical perturbing element which is a dielectric curled micro-bimorph cantilever. Further detailed research is carried out on fabrication technology for monolithic integration of micro-cantilevers with the aforementioned optical devices. Finally the thesis presents the mechano-optical characterisation of the integrated devices.

Galerkin based static and dynamic models are developed to predict various properties of curled micro-bimorph cantilevers; such as pull-in voltage, resonance frequency and shift in resonance frequency by electrostatic spring softening. The model predictions on pull-in voltage matched with the experimental data on curled cantilevers with a relative mean error of $6 \%$. However, the model predictions carried out on integrated devices (on SOI wafers) show a deviation of $\sim 30 \%$ compared to the measured data. To understand the discrepancy between the model predictions and the measurements further investigation has to be carried out. The effects of the silicon device layer on the electrostatic actuation and the effect of damping on the (dynamic) pull-in voltage need further investigation. The effects of temperature on the off-state deflection and long-term charging effects on the performance of the cantilever have not been studied and the models need to be improved to study some of the aforementioned effects.

The thesis describes a successful fabrication technology for monolithic integration of curled micro-bimorph cantilevers with micro-ring resonators and photonic crystals. The process utilises conventional lithography with simple two mask (for MRR) and three mask (for photonic crystals) fabrication schemes. It has to be noted that the devices discussed in the thesis are the outcome of the first design considered. Two batches were fabricated in which the first batch (with MRR) uses a sacrificial layer thickness of $196 \mathrm{~nm}$ (measured) whereas the second batch deals with a reduced sacrificial layer thickness of $105 \mathrm{~nm}$ (measured). The latter scheme realises tips that are self-aligned with respect to the holes of the photonic crystals while not needing precise alignment of any mask with respect to the nano-sized PC holes. By carefully selecting the sacrificial layer thickness with respect to the PC hole diameters this technology aids to create these self-aligned tips with varying diameter and length. 
Another important achievement of this research is the conversion of the final integrated PC slab into air bridge membranes, by locally removing the lower cladding layer. This results in PC slabs with a symmetric cladding, preventing coupling between TE-like and TM-like modes.

As the sacrificial layer thickness is one of the parameters that determines the airgap between the micro-cantilever and the optical device and since any mechano-optic modulation requires this gap to be small, it is desirable to limit the thickness of the sacrificial layer to less than the evanescent field depth. However the minimum sacrificial layer thickness is restricted by the etch selectivity between adjacent layers, making it a pivotal choice in the fabrication process. Further the non-uniformity of the deposited layers increases the process complexity: over-etching can seriously risk the yield of the process, as it removes the protective layers of the access waveguides.

The final integrated devices were accompanied by unwanted etch residues called stringers. Two novel methods for stringer elimination are proposed in this thesis. The first method uses the reshaping of an intermediate layer on top of the topographic pattern to relax the selectivity limitation which is a major cause for the appearance of stringers. The second method utilises acoustic waves produced in an ultrasonic cleaner to mechanically agitate and remove released stringers. The second method is successfully utilised to fabricate functional devices.

Mechano-optical characterisation of the micro-cantilever integrated micro-ring resonator is demonstrated in chapter five. By operating a $40 \mu \mathrm{m}$ long cantilever in its static bending mode, the resonance wavelength of the micro-ring resonator could be tuned by $122 \mathrm{pm}$ with a modulation depth of $18 \mathrm{~dB}$. The device performance does not suffer from any losses over the stable range of the cantilever operation. The wavelength tunability was restricted to $122 \mathrm{pm}$, larger tuning voltages resulting in pull-in of the cantilever (followed by subsequent permanent stiction to the optical device layer). Measured mechanical quality factors in dependence of pressure showed that at atmospheric pressure the cantilever is near-critically damped yielding a faster system compared to when it is either over- or under-damped. Time response of the cantilever measured by Laser Doppler Vibrometer showed a signal rise and fall time of $\sim 2.5 \mu \mathrm{s}$ when driven by a $2 \mathrm{kHz}$ square wave drive signal of 0-2 V. However the dynamic optical switching measurement (square wave drive signal of 0-4 V) with a $100 \mu \mathrm{m}$ long cantilever showed a signal rise time of $\sim 16 \mu$ s and fall time of $\sim 5 \mu$ s. The maximum actuation frequency for this device was limited to $30 \mathrm{kHz}$ due to the increased rise time possibly introduced by the softening of the cantilever during voltage controlled actuation, even 
though the cantilever showed a measured first mode resonance frequency of $\sim 140 \mathrm{kHz}$ (at rather small applied AC voltage of $0.04 \mathrm{~V}$ and DC voltage of $0.5 \mathrm{~V}$ ).

Optical modulation measurements performed on a PCS-WG with integrated micro-cantilever yielded $80 \%$ modulation at $1 \mathrm{kHz}(0-8 \mathrm{~V}$ square wave drive signal applied to a $40 \mu \mathrm{m}$ cantilever). It is noteworthy that this modulation is obtained on a mechano-optical interaction area as small as $10 \times 10 \mu \mathrm{m}^{2}$. Further band edge tuning is demonstrated in a PCS-MC with an integrated micro-cantilever. At its Out1 port, on application of a DC voltage as small as $0.75 \mathrm{~V}$ the third band edge was shifted by $560 \mathrm{pm}$ with $21 \mathrm{~dB}$ modulation. Similar measurements are performed in the second band edges and on the Out2 port.

The major conclusions from the research carried out in this thesis can be summarised as,

- A simple fabrication technology for monolithic integration of micro-cantilevers with silicon based optical devices is developed.

- The integrated mechanical element is actuated at low voltages (pull-in voltage of the cantilevers range from $3 \mathrm{~V}$ to $9 \mathrm{~V}$ ), which is one of the requirements for CMOS compatibility.

- In the fabrication process the integrated photonic crystal based devices are converted into air-bridge membranes where symmetry in the cladding is achieved.

- Substantial optical modulation (18 dB in MRR and $21 \mathrm{~dB}$ in PCS-MC) is demonstrated.

- No insertion loss is observed in the MRR during the stable range of operation of the micro-cantilever.

- $80 \%$ throughput modulation is observed in a PCS-WG at $1 \mathrm{KHz}$ with a $0-8 \mathrm{~V}$ square wave signal on a device of only $10 \mu \mathrm{m}$ long.

- The bandwidth of the integrated device is greater than $30 \mathrm{kHz}$.

\subsection{Outlook}

This thesis demonstrates successful integration and mechano-optic characterisation of wavelength selective devices with integrated, electrostatically actuated microcantilevers. However there are many ways the device performance can be improved. This section briefly underlines the possibilities for further exploration towards this goal.

One of the major problems observed during mechanical characterisation of the integrated device was the observation of a clear pull-in at a voltage lower than anticipated from our models. As the cantilever got permanently stuck to the silicon device layer after pull-in, a further improvement in cantilever design is required to reduce stiction after pull- 
in. Towards this aim, an improved design is proposed in chapter five by including holes in the micro-cantilever. In addition to preventing stiction, this design can provide an additional advantage in reduction of the sacrificial layer etch time. However it has to be noted that during metal electrode wet etching, care has to be taken not to introduce large undercut. Another option can be to use PECVD (plasma enhanced chemical vapour deposition) layers for the cantilever and the sacrificial layer exploiting increased surface roughness compared to LPCVD layers. Roughness in the layer can reduce the contact area between the surfaces. Low-temperature PECVD deposited layers have additional advantage of CMOS compatibility. However in that case, the process needs to be further optimised considering the change in material properties introduced by the process variation. Selection of gold as the upper electrode also restricts CMOS compatibility of the device. Gold is selected by virtue of its low residual stress, which results in limited offstate deflection. A promising alternative candidate can be aluminium, which on the other hand will require a careful selection of the sacrificial layer. Higher tunability in the integrated device can be achieved by increasing the interacting area between the cantilever and the micro-ring resonator. This requires a design improvement, where the cantilever covers larger circumference of the ring. However care has to be taken in the design such that the cantilever does not affect the designed coupling strength of the MRR, which otherwise can result in a reduced optical quality factor.

An experimental investigation on the efficiency of the stringer removal mechanism utilising 'reshaping of intermediate layers' is recommended. Further the stringer removal mechanism by sonification opens a scope for fundamental research on ultrasonic cavitation and subsequent erosion in micro machined devices.

One of the important results of this research is the successful realisation of a technology for fabricating self-aligned nano-tips. Apart from the optical regime, this fabrication can have ample application in microfluidics especially in the case of micro-or nano sieves in which a self aligned tip from a mechanical element can be used for its selective opening/closing. In the case of PC slabs, the research can be extended to PC slab based Y-splitters [1] for realisation of wavelength selective switching between its output ports. The technology can be utilised if the PC slab holes are designed in such a way that the hole diameter is higher for cavity holes than for normal slab holes. Thus the process can be tuned for fabrication of self-aligned tip with respect to a specific cavity hole. On actuation, this can result in variation of the cavity geometry at the PC slab Yjunction. 
Chapter 7. Conclusions and outlook

In short, successful realisation of three different types of compact integrated wavelength selective devices is demonstrated. The technology opens new avenues for further exploration towards realisation of CMOS compatible optical modulators.

\subsection{Reference}

[1] L. J. Kauppinen, et al., "Photonic crystal cavity-based Y splitter for mechano-optical switching," IEEE Photonics Technology Letters, vol. 22, pp. 966-968, 2010. 


\section{Summary}

This thesis deals with the fabrication technology and mechano-optical characterisation of compact integrated wavelength selective optical devices for use in telecommunication applications. Upon electrostatic actuation, a mechanical element perturbs the optical evanescent field of a guided wave providing electro mechano-optical modulation of light. The silicon-on-insulator (SOI) based optical devices are fabricated in a silicon photonics fabrication platform called ePIXfab using deep-UV lithography. Further, using surface micromaching techniques, the mechanical elements are monolithically integrated with the optical devices in the MESA+ Cleanroom. Mechanooptical modulation is investigated in three different types of optical devices, viz. a microring resonator, a photonic crystal slab waveguide and a photonic crystal microcavity based device. Owing to their ease of fabrication and low power consumption, electrostatically actuated curled micro-bimorph cantilevers are chosen as the mechanical perturbing element. The thesis concentrates on the characterisation of the mechanical elements, the development of integration technologies and mechano-optical characterisation of the integrated devices.

After introducing the purpose of the research, the first chapter explains the importance of matured silicon based technology for realising integrated optical devices. Further chapter two presents a review on the various optical modulation schemes, realised so far in literature. The relative merits of mechano-optical modulation schemes over existing alternative techniques such as those based on thermo-optic effects, electrooptic effects, plasma dispersion effects and liquid crystal infiltration are discussed.

A detailed analysis of the mechanical characterisation of curled micro-bimorph cantilevers is carried out in chapter three. The off-state tip deflections of the microcantilevers are optimised to an acceptable distance of $<500 \mathrm{~nm}$ above the optical device, by considering various parameters such as length of the cantilever, upper electrode thickness and material. Further Galerkin based static and dynamic models are developed to predict various mechanical parameters of the curled micro-cantilever such as pull-in voltage, resonance frequency and resonance frequency shift by electrostatic spring softening. The predictions are validated against measured data.

A detailed explanation for a two mask fabrication scheme for integration of microcantilevers with race-track type micro-ring resonators is given in chapter four. Various fabrication related issues, of which a major one is related with the formation of stringers (unwanted residues left in the corners of a stepped surface), are addressed in this chapter. After discussing various existing stringer elimination methods, two novel alternative 
methods are proposed. Further the chapter demonstrates successful device realisation using one of these methods: i.e. utilising ultrasonic cleaning. The next chapter provides the mechano-optical characterisation of the micro-ring resonators integrated with microcantilevers. The mechanical characterisation includes analyses of both static and dynamic parameters such as off-state tip deflection, pull-in voltage, first order mode resonance frequency, quality factor, frequency shift by electrostatic spring softening etc. The device is near-critically damped, thus providing the fastest possible response for a device of given resonance frequency. Static optical tuning measurements demonstrate a wavelength shift of $122 \mathrm{pm}$ and modulation depth of $18 \mathrm{~dB}$ for a $40 \mu \mathrm{m}$ cantilever whereas dynamic measurements demonstrate optical switching with a rise time of $16 \mu$ s for a $100 \mu \mathrm{m}$ cantilever.

A fabrication technology for integration of micro-cantilevers equipped with selfaligned tips with respect to the holes of a photonic crystal slab waveguide and photonic crystal micro-cavity based device is introduced in chapter six. Further both devices are mechano-optically characterised. An $80 \%$ throughput modulation in a photonic crystal slab waveguide is demonstrated. More importantly $560 \mathrm{pm}$ wavelength tuning with $21 \mathrm{~dB}$ modulation in the third band edge of a photonic crystal microcavity based device is demonstrated. Finally chapter seven concludes the research described in the thesis with a discussion on possible future research. 


\section{Samenvatting}

Dit proefschrift beschrijft de fabricage en karakterisatie van compact geïntegreerde golflengteselectieve optische componenten voor toepassing in de telecommunicatie. De werking is gebaseerd op een elektrostatisch geactueerd mechanisch element, dat het evanescente veld in een optische component verstoord. Hierdoor is het mogelijk om licht op mechanische wijze te moduleren. De op 'silicon-on-insulator' (SOI) gebaseerde optische componenten zijn gerealiseerd in een fabricageplatform genaamd ePIXfab, waarbij gebruik wordt gemaakt van diep-ultraviolet licht. De mechanische elementen voor de optische modulatie worden middels surface micromachining in de MESA+ Cleanroom aangebracht. De mechano-optische modulatie is bestudeerd voor drie optische componenten: een microring-resonator, een planaire golfgeleider met fotonisch kristal en een op een fotonisch kristal gebaseerde micro-resonator. Vanwege de relatief eenvoudige realisatie en het lage energieverbruik is er gekozen voor elektrostatisch aangedreven micro-kraagbalkjes voor optische modulatie. Dit proefschrift beschouwt de karakterisatie van het mechanische element, de mogelijke technieken voor integratie van dit element in een optische component en de mechano-optische karakterisatie van de gerealiseerde geïntegreerde structuren.

In het eerste hoofdstuk wordt het belang van standaard silicium fabricage technieken toegelicht voor de realisatie van geïntegreerde optische componenten. In het tweede hoofdstuk worden de tot dusver in de literatuur beschreven modulatiemethodes besproken. Hierbij wordt een vergelijking gemaakt tussen de mechano-optische methode en de modulatietechnieken waarbij gebruik wordt gemaakt van het thermo-optische effect, het elektro-optische effect, het plasma-dispersie effect en van vloeibare-kristal infiltratie.

Hoofdstuk drie geeft een uitvoerige analyse van de mechanische eigenschappen van de gerealiseerde bimorph micro kraagbalk. De uitwijking van de micro kraagbalk in rustpositie is geoptimaliseerd tot een afstand van minder dan $500 \mathrm{~nm}$ tot de optische component. Belangrijke parameters hierbij zijn de lengte van de cantilever, de dikte van de bovenste elektrode, het gebruikte materiaal en bijbehorende depositie. Om eigenschappen als 'pull-in' spanning, resonantiefrequentie en de verschuiving van de resonantiefrequentie door elektrostatische 'veer-veslapping' te voorspellen is er een op een Galerkin methode gebaseerd statisch en dynamisch model ontwikkeld. De resultaten van dit model zijn vergeleken met de verkregen meetdata.

Hoofdstuk vier geeft een gedetailleerde beschrijving van het fabricageproces voor de integratie van een microcantilever in een 'race-track' microring-resonator. Diverse 
fabricage-gerelateerde problemen worden beschreven, waaronder het ontstaan van zogenaamde stringers - een ongewenst residu dat achterblijft na het etsen. Naast een toelichting op de bestaande methodes om deze stringers te elimineren worden ook twee nieuwe methodes voorgesteld. Verder wordt de fabricage van micro-kraagbalken geïntegreerd met microring-resonatoren beschreven waarbij gebruik wordt gemaakt van één van de voorgestelde nieuwe methodes voor het verwijderen van de stringers, namelijk ultrasoon reinigen. Het daarop volgende hoofdstuk beschrijft de mechano-optische karakterisatie van de microring-resonatoren met geïntegreerde micro-kraagbalken. Hierbij zijn zowel de statische als de dynamische eigenschappen gekarakteriseerd, waaronder uitwijking van de tip in rustpositie, 'pull-in' spanning, eerste orde resonantiefrequentie, kwaliteitsfactor en verschuiving in resonantiefrequentie door elektrostatsiche 'veerverslapping'. Uit metingen volgt dat er een statisch optische verstemming met een verschuiving van $122 \mathrm{pm}$ in de golflengte en een modulatiediepte van $18 \mathrm{~dB}$ haalbaar is. Eerste dynamische metingen laten een stijgtijd van $16 \mu$ s voor een kraagbalk van $100 \mu \mathrm{m}$ zien.

In hoofdstuk zes wordt de fabricagetechnologie beschreven om een kraagbalk met tips te realiseren waarbij deze precies uitgelijnd zijn ten opzichte van de gaten in een fotonisch kristal. Deze optische componenten zijn gekarakteriseerd waarbij een modulatie van $80 \%$ gemeten is bij een planaire golfgeleider met een fotonisch kristal. Bij een op fotonisch kristal gebaseerde micro-resonator is door middel van mechano-optische verstemming een verschuiving in de golflengte van $560 \mathrm{pm}$ gemeten en een modulatie van $21 \mathrm{~dB}$ in de derde 'band-flank' van het fotonisch kristal. Hoofdstuk zeven sluit af met een discussie over toekomstig onderzoek. 
Appendices 


\section{T. Freeze-Drying Procedure}

Standard procedure available at $\mathrm{MESA}^{+}$Cleanroom [2] database

Parameters are valid for $100 \mathrm{~mm}$ wafers or for smaller samples

- $200 \mathrm{ml}$ buffered hydrofluric acid (BHF, $\mathrm{NHF}_{4} / \mathrm{HF} 1: 7$ )

-- choice of etchant is defined by design

- Rinse with 6 litres of DI-water, time 30 min

- Dilute DI with $800 \mathrm{ml}$ IPA, time $15 \mathrm{~min}$

- Transport sample in $200 \mathrm{ml}$ IPA, time $30 \mathrm{~min}$

- Dilute IPA with $600 \mathrm{ml}$ cyclohexane, time $15 \mathrm{~min}$

- Transport sample in $100 \%$ cyclohexane I, $200 \mathrm{ml}$, time $15 \mathrm{~min}$

- Transport sample in 100\% cyclohexane II $200 \mathrm{ml}$, time $15 \mathrm{~min}$

- Freeze drying: 0.5 Bar dry nitrogen $\left(\mathrm{N}_{2}\right)$, electrode temperatures of $-5^{\circ} \mathrm{C}$

- Total process time: $135 \mathrm{~min}$, (excluding the sacrificial layer etch time)

\section{Procedure used for the processes described in this thesis}

1. Rinse all vessels with IPA in order to remove possible contaminations and dry them with $\mathrm{N}_{2}$ gas.

2. Use $200 \mathrm{ml}$ of BHF (VLSI-Etch mixture AF, 87.5-12.5) in a plastic beaker. Place the wafer in it for etching (etching time is dependant on the process).

3. As soon as it approaches the required etch time, start rinsing with DI water to remove the BHF etchant from the beaker. Rinse for 30 minutes with DI water.

4. After 30 minutes stop the DI water flow and pour off $100 \mathrm{ml}$ water from beaker to outside, keeping the rest of the water inside the beaker.

5. Pour $100 \mathrm{ml}$ IPA (2- propanol-VLSI), into the beaker through a funnel and wait for 2 minutes.

6. Pour off $100 \mathrm{ml}$ of the liquid (IPA+DI water) from the beaker and pour-in $100 \mathrm{ml}$ IPA into the beaker. Repeat step 5 and 6 at least 6 times to make it close to $100 \%$ of IPA.

7. Pour $200 \mathrm{ml}$ IPA into another beaker and transfer the wafer into it for $>20$ minutes. (care has to be taken such that the side of the wafer containing released devices should hold enough liquid)

8. Transfer the wafer with the liquid (IPA + DI water) on top of it, to a beaker containing $200 \mathrm{ml}$ cyclohexane, for $>25$ minutes. 
9. Transfer the wafer into a second beaker containing $200 \mathrm{ml}$ of cyclohexane for $>25$ minutes.

10. Transfer the wafer with cyclohexane on top of it to a clean room tissue paper to remove the cyclohexane from the backside of the wafer.(Ice may disturb the thermal contact between wafer and the chuck).

11. Keep the chuck at $-5^{\circ} \mathrm{C}$ for $>30$ minutes (The freezing point of cyclohexane is at $6.7^{\circ} \mathrm{C}$ ). Transfer the wafer into the chuck and wait for a few seconds for the ice to develop on it, before closing the glass hood.

12. Wait for approximately 10 minutes, until it is seen that the ice is gone from the top of the wafer. Again wait for another $>30$ minutes to remove the ice beneath the released structures.

13. Heat the reactor to room temperature, to avoid moisture condensation on the wafer, and keep rinsing with $\mathrm{N}_{2}$ flow.

14. After rinsing for 20 minutes at room-temperature, stop the $\mathrm{N}_{2}$ flow and open the furnace to inspect the wafer.

\section{Apparatus control}

1. Connect-Water cooling for the Peltier chuck.

2. Connect-Electrical connectors for the chuck.

3. Connect-Thermocouple.

4. Connect $-\mathrm{N}_{2}$ flow to the reactor.

5. Connect-Power supply.

6. Start with rinsing reactor and the chuck with $\mathrm{N}_{2}$ flow (0.5 bar, at $25^{\circ} \mathrm{C}$ for 30 minutes).

7. Cool down the reactor to $-5^{\circ} \mathrm{C}$ for $>30$ minutes; keep rinsing with $\mathrm{N}_{2}$ flow.

[1] R. Legtenberg and H. A. C. Tilmans, "Electrostatically driven vacuumencapsulated polysilicon resonators Part I. Design and fabrication," Sensors and Actuators A, vol. 45, pp. 57-66, 1994.

[2] $\quad M E S A^{+}$Cleanroom, University of Twente, The Netherlands. Available: http://www.mesacrl.utwente.nl/mis/MISHomePage.htm 


\section{Process Documents}

Table I. The process document for micro-cantilever fabrication (chapter 3)

\begin{tabular}{|c|c|c|c|}
\hline Step & Process & Parameters & Remarks \\
\hline 01 & $\begin{array}{l}\text { Substrate selection - } \\
\text { Silicon }<100>\text { Double } \\
\text { Side Polished (DSP) } \\
\text { (low resistance) }\end{array}$ & $\begin{array}{l}\text { Orientation: }<100> \\
\text { Diameter: } 100 \mathrm{~mm} \\
\text { Thickness: } 525+/-25 \mu \mathrm{m} \\
\text { Polished: Double side } \\
\text { Resistivity: } 0.010-0.015 \Omega \cdot \mathrm{cm} \\
\text { Type: p }\end{array}$ & $\begin{array}{l}\text { To be used as the } \\
\text { bottom electrode } \\
\text { Use at least } 5 \text { dummy } \\
\text { wafers (Single Side } \\
\text { Polished (SSP)) }\end{array}$ \\
\hline 02 & $\begin{array}{l}\text { Wafer thickness } \\
\text { measurement }\end{array}$ & HeidenHahn & \\
\hline 03 & Cleaning Standard & $\begin{array}{l}\mathrm{HNO}_{3}(99 \%) \text { Selectipur: MERCK } \\
\mathrm{HNO}_{3}(69 \%) \text { VLSI: MERCK } \\
\text { - Beaker 1: fumic } \mathrm{HNO}_{3}(99 \%), 5 \mathrm{~min} \\
\text { - Beaker 2: fumic } \mathrm{HNO}_{3}(99 \%), 5 \mathrm{~min} \\
\text { - Quick Dump Rinse }<0.1 \mu \mathrm{S} \\
\text { - Beaker 3: boiling }\left(95^{\circ} \mathrm{C}\right) \mathrm{HNO}_{3}(69 \%), 10 \\
\text { min } \\
\text { - Quick Dump Rinse }<0.1 \mu \mathrm{S} \\
\text { - Spin drying }\end{array}$ & $\begin{array}{l}\text { Start from beaker } 2 \\
\text { (as it is fresh wafers) }\end{array}$ \\
\hline 04 & $\begin{array}{l}\text { Wet Oxidation (WOX) } \\
\text { at } 1150^{\circ} \mathrm{C} \text { of Silicon }\end{array}$ & $\begin{array}{l}\text { Standby temperature: } 800{ }^{\circ} \mathrm{C} \\
\text { Check water level of bubbler } \\
\text { - Program: WOX-1150 } \\
\text { - Temp.: } 1150{ }^{\circ} \mathrm{C} \\
\text { - Gas: } \mathrm{H}_{2} \mathrm{O}+\mathrm{N}_{2} \text { (Bubbler) }\end{array}$ & $\begin{array}{l}\text { Thickness } 1500 \mathrm{~nm} \\
\text { Time } \sim 04.57 \mathrm{hr}: \mathrm{min} . \\
\text { Use at least } 6 \text { dummy } \\
\text { wafers (SSP) } \\
\text { (See Figure } 3.6 \text { a) }\end{array}$ \\
\hline 05 & $\begin{array}{l}\text { Ellipsometer } \\
\text { Measurement }\end{array}$ & Metrology Woollam ellipsometer & $\begin{array}{l}\text { For measuring layer } \\
\text { thickness }\end{array}$ \\
\hline 06 & $\begin{array}{l}\text { Wafer curvature } \\
\text { measurement }\end{array}$ & $\begin{array}{l}\text { Veeco Dektak } 8 \\
\text { To measure the stress in the deposited layer }\end{array}$ & $\begin{array}{l}\text { Measure at } 0^{\circ}, 45^{\circ}, 90^{\circ} \\
\text { and at } 135^{\circ} \text { on silicon } \\
\text { dummy wafers and use } \\
\text { them in steps } 07,08 \text { and } \\
10 \mathrm{~A} \text {. }\end{array}$ \\
\hline 07 & Cleaning Standard & $\begin{array}{l}\mathrm{HNO}_{3}(99 \%) \text { Selectipur: MERCK } \\
\mathrm{HNO}_{3}(69 \%) \text { VLSI: MERCK } \\
\text { - Beaker 1: fumic } \mathrm{HNO}_{3}(99 \%), 5 \mathrm{~min} \\
\text { - Beaker 2: fumic } \mathrm{HNO}_{3}(99 \%), 5 \mathrm{~min} \\
\text { - Quick Dump Rinse }<0.1 \mu \mathrm{S} \\
\text { - Beaker 3: boiling }\left(95^{\circ} \mathrm{C}\right) \mathrm{HNO}_{3}(69 \%), 10 \\
\text { min } \\
\text { - Quick Dump Rinse }<0.1 \mu \mathrm{S} \\
\text { - Spin drying }\end{array}$ & \\
\hline
\end{tabular}




\begin{tabular}{|c|c|c|c|}
\hline 08 & $\begin{array}{l}\text { LPCVD } \operatorname{SiN}_{x}-\text { low } \\
\text { stress }\end{array}$ & $\begin{array}{l}\text { Tempress LPCVD/HC } \\
\text { Tube: G3 } \\
\text { - } \mathrm{SiH}_{2} \mathrm{Cl}_{2} \text { flow: } 70 \mathrm{sccm} \\
\text { - } \mathrm{NH}_{3} \text { flow: } 18 \mathrm{sccm} \\
\text { - Temperature: } 830{ }^{\circ} \mathrm{C} \\
\text { - Pressure: } 200 \mathrm{~m} \text { Torr } \\
\text { - Program: SiRN01 } \\
\text { Deposition rate: } 3.9 \sim 5.3 \mathrm{~nm} / \mathrm{min} \\
\mathrm{N}_{\mathrm{f}} \cdot 2.14\end{array}$ & $\begin{array}{l}\text { Thickness } \sim 1.0 \mu \mathrm{m} \\
\text { Time } \sim 04: 10 \text { hr:min } \\
\text { Use at least } 6 \text { dummy } \\
\text { wafers (SSP). } \\
\text { (See Figure } 3.6 \mathrm{~b} \text { ) }\end{array}$ \\
\hline 09 & $\begin{array}{l}\text { Ellipsometer } \\
\text { Measurement }\end{array}$ & Metrology Woollam ellipsometer & $\begin{array}{l}\text { For measuring layer } \\
\text { thickness }\end{array}$ \\
\hline 10 & $\begin{array}{l}\text { Wafer curvature } \\
\text { measurement }\end{array}$ & $\begin{array}{l}\text { Veeco Dektak } 8 \\
\text { (A) For SiN } \mathbf{x}_{\mathbf{x}} \text { layer: Use the silicon wafers } \\
\text { measured in step 06. Etch the } \mathrm{SiN}_{\mathrm{x}} \text { on the back } \\
\text { side using step 25. And measure the final wafer } \\
\text { curvature. } \\
\text { (B) For } \mathrm{Cr} \text { layer: Use new silicon dummy } \\
\text { wafers and measure the initial wafer curvature. }\end{array}$ & $\begin{array}{l}\text { Measure the wafer } \\
\text { curvature at } 0^{\circ}, 45^{\circ}, 90^{\circ} \\
\text { and at } 135^{\circ} \text {. }\end{array}$ \\
\hline 11 & Cleaning Standard & $\begin{array}{l}\mathrm{HNO}_{3}(99 \%) \text { Selectipur: MERCK } \\
\mathrm{HNO}_{3}(69 \%) \text { VLSI: } \mathrm{MERCK} \\
\text { - Beaker 1: fumic } \mathrm{HNO}_{3}(99 \%), 5 \mathrm{~min} \\
\text { - Beaker 2: fumic } \mathrm{HNO}_{3}(99 \%), 5 \mathrm{~min} \\
\text { - Quick Dump Rinse <0.1 } \mu \mathrm{S} \\
\text { - Beaker 3: boiling }\left(95^{\circ} \mathrm{C}\right) \mathrm{HNO}_{3}(69 \%), 10 \\
\text { min } \\
\text { - Quick Dump Rinse <0.1 } \mathrm{MS} \\
\text { - Spin drying }\end{array}$ & \\
\hline 12 & Sputtering of $\mathrm{Cr}$ & $\begin{array}{l}\text { Sputterke } \\
\text { - Electrode temperature : Water cooled } \\
\text { electrode } \\
\text { - Power: } 200 \mathrm{~W} \\
\text { - Base Pressure: }<1 \text { X } 10^{-6} \mathrm{mbar} \\
\text { - Sputter Pressure: }<6.6{\mathrm{X} 10^{-3} \mathrm{mbar}} \\
\text { Deposition rate }(100 \mathrm{~mm} \text { wafer): } 15 \mathrm{~nm} / \mathrm{min} \\
\text { Thickness to be deposited: } 50 \mathrm{~nm}, 100 \mathrm{~nm} \text {, } \\
150 \mathrm{~nm} \text { and } 200 \mathrm{~nm}\end{array}$ & $\begin{array}{l}\text { * Sputter also on } \\
\text { dummy wafers with a } \\
\text { scotch tape on it to } \\
\text { measure the layer } \\
\text { thickness by Dektak. } \\
\text { (See Figure } 3.6 \text { c) }\end{array}$ \\
\hline 13 & $\begin{array}{l}\text { Wafer curvature } \\
\text { measurement }\end{array}$ & $\begin{array}{l}\text { Veeco Dektak } 8 \\
\text { Use the silicon dummy wafers used in step } 10 \\
B .\end{array}$ & $\begin{array}{l}\text { Measure at } 0^{\circ}, 45^{\circ}, 90^{\circ} \\
\text { and at } 135^{\circ} .\end{array}$ \\
\hline 14 & $\begin{array}{l}\text { Layer Thickness } \\
\text { Measurement }\end{array}$ & Veeco Dektak 8 & $\begin{array}{l}\text { Remove the scotch tape } \\
\text { from dummy wafers } \\
\text { and measure the step } \\
\text { height. }\end{array}$ \\
\hline 15 & $\begin{array}{l}\text { Lithography - Priming } \\
\text { (liquid) }\end{array}$ & $\begin{array}{l}\text { HexaMethylDiSilazane (HMDS) } \\
\text { - Dehydration bake }\left(120^{\circ} \mathrm{C}\right): 5 \mathrm{~min} \\
\text { - Spin program: } 5(5000 \mathrm{rpm}, 20 \mathrm{sec})\end{array}$ & \\
\hline
\end{tabular}




\begin{tabular}{|c|c|c|c|}
\hline 16 & $\begin{array}{l}\text { Lithography - Coating } \\
\text { Olin907-35 }\end{array}$ & $\begin{array}{l}\text { - Spinning acceleration: } 4000 \mathrm{rpm} / \mathrm{s} \\
\text { - Spinning speed: } 4000 \mathrm{rpm} \\
\text { - Spinning time: } 20 \mathrm{~s} \\
\text { - Prebake }\left(95^{\circ} \mathrm{C}\right): 90 \mathrm{~s}\end{array}$ & $\begin{array}{l}\text { Wait } 30 \text { min before } \\
\text { exposure to avoid } \\
\text { stiction }\end{array}$ \\
\hline 17 & $\begin{array}{l}\text { Lithography - } \\
\text { Alignment \& Exposure } \\
\text { Olin } 907-35 \text { (EVG 20) }\end{array}$ & $\begin{array}{l}\text { Electronic Vision Group } 20 \text { Mask Aligner } \\
\text { - Hg-lamp: } 12 \mathrm{~mW} / \mathrm{cm}^{2} \\
\text { - Exposure Time: } 9.3 \mathrm{sec}\end{array}$ & $\begin{array}{l}\text { Mask: For patterning } \\
\text { SiN }_{\mathrm{x}} \text { cantilever layer } \\
\text { Hard contact }\end{array}$ \\
\hline 18 & $\begin{array}{l}\text { Lithography - } \\
\text { Development Olin } \\
\text { Resist }\end{array}$ & $\begin{array}{l}\text { Developer: OPD4262 } \\
\text { - After Exposure Bake }\left(120{ }^{\circ} \mathrm{C}\right): 60 \mathrm{sec} \\
\text { Development: } \\
\text { - Time: } 30 \mathrm{sec} \text { in Beaker } 1 \\
\text { - Time: } 15-30 \mathrm{sec} \text { in Beaker } 2 \\
\text { - Quick Dump Rinse }<0.1 \mu \mathrm{S} \\
\text { - Spin drying }\end{array}$ & $\begin{array}{l}\text { Wait at least } 30 \mathrm{~min} \\
\text { before post bake }\end{array}$ \\
\hline 19 & $\begin{array}{l}\text { Optical microscopic } \\
\text { inspection-Lithography }\end{array}$ & Microscope & \\
\hline 20 & $\begin{array}{l}\text { Lithography - Post } \\
\text { bake standard }\end{array}$ & Hot plate at $120^{\circ} \mathrm{C}: 30 \mathrm{~min}$ & \\
\hline 21 & $\begin{array}{l}\text { Ozone anneal of Olin } \\
907-35\end{array}$ & $\begin{array}{l}\text { UV PRS-100 } \\
\text { - To improve wetting during etching } \\
\text { - Time: } 300 \mathrm{sec}\end{array}$ & \\
\hline 22 & Wet etching of $\mathrm{Cr}$ & $\begin{array}{l}\text { Chromium etch LSI selectipur : MERCK } \\
111547.2500 \\
\text { - Quick Dump Rinse }<0.1 \mu \mathrm{S} \\
\text { - Spin drying } \\
\text { Etch rate }=0.5 \mathrm{~nm} / \mathrm{min}\end{array}$ & $\begin{array}{l}\text { The etch time depends } \\
\text { on the Cr thickness } \\
\text { (See Figure } 3.6 \mathrm{~d} \text { ) }\end{array}$ \\
\hline 23 & $\begin{array}{l}\text { Optical microscopic } \\
\text { inspection-Lithography }\end{array}$ & Microscope & \\
\hline 24 & $\begin{array}{l}\text { Plasma cleaning (Etske) } \\
\text { Cleaning }\end{array}$ & $\begin{array}{l}\text { Elektrotech PF310/340 } \\
\text { Dirty chamber } \\
\text { Styros electrode } \\
\text { - Electrode temp.: } 10{ }^{\circ} \mathrm{C} \\
\text { - } \mathrm{O}_{2} \text { flow: } 20 \mathrm{sccm} \\
\text { - Pressure: } 0 \mathrm{mTorr} \\
\text { - Power: } 150 \mathrm{~W}\end{array}$ & $\begin{array}{l}\text { Removal of Residues } \\
\text { Time: 10:00 min:sec }\end{array}$ \\
\hline 25 & $\begin{array}{l}\text { Plasma etching } \mathrm{SiN}_{\mathrm{x}} \\
\text { (Etske) }\end{array}$ & $\begin{array}{l}\text { Elektrotech PF310/340 } \\
\text { Dirty chamber } \\
\text { Styros electrode } \\
\text { - Electrode temp.: } 10^{\circ} \mathrm{C} \\
\text { - } \mathrm{CHF}_{3} \text { flow: } 25 \mathrm{sccm} \\
\text { - } \mathrm{O}_{2} \text { flow: } 5 \mathrm{sccm} \\
\text { - Pressure: } 10 \mathrm{mTorr} \\
\text { - Power: } 75 \mathrm{~W} \\
\text { Etchrate } \mathrm{SiN}_{\mathrm{x}}=55 \mathrm{~nm} / \mathrm{min}\left(\text { for } \mathrm{V}_{\mathrm{DC}}=-460 \mathrm{~V}\right) \\
\text { Etchrate Olin resist }=95 \mathrm{~nm} / \mathrm{min} \\
\text { If DC-Bias }<375 \mathrm{~V} \text { apply chamber clean }\end{array}$ & $\begin{array}{l}\text { Backside } \\
\text { For } 1.0 \mu \mathrm{m} \mathrm{SiN} \text {. } \\
\text { Etch time }=25 \text { min. } \\
\text { Due to non-uniformity } \\
\text { in the deposited layer, } \\
\text { overetching has been } \\
\text { performed to } \\
\text { completely remove the } \\
\text { SiN }_{\mathrm{x}}\end{array}$ \\
\hline
\end{tabular}




\begin{tabular}{|c|c|c|c|}
\hline 26 & $\begin{array}{l}\text { Plasma etching } \operatorname{SiN}_{x} \\
\text { (Etske) }\end{array}$ & $\begin{array}{l}\text { Elektrotech PF310/340 } \\
\text { Dirty chamber } \\
\text { Styros electrode } \\
\text { - Electrode temp.: } 10{ }^{\circ} \mathrm{C} \\
\text { - } \mathrm{CHF}_{3} \text { flow: } 25 \mathrm{sccm} \\
\text { - } \mathrm{O}_{2} \text { flow: } 5 \mathrm{sccm} \\
\text { - Pressure: } 10 \mathrm{mTorr} \\
\text { - Power: } 75 \mathrm{~W} \\
\text { Etchrate } \mathrm{SiN}_{\mathrm{x}}=55 \mathrm{~nm} / \mathrm{min}\left(\text { for } \mathrm{V}_{\mathrm{DC}}=-460 \mathrm{~V}\right) \\
\text { Etchrate Olin resist }=95 \mathrm{~nm} / \mathrm{min} \\
\text { If DC-Bias }<375 \mathrm{~V} \text { apply chamber clean }\end{array}$ & $\begin{array}{l}\text { Front side } \\
\text { For } 1.0 \mu \mathrm{m} \mathrm{SiN}, \\
\text { including the } \\
\text { overetching, etch } \\
\text { time }=25 \mathrm{~min}\end{array}$ \\
\hline 27 & $\begin{array}{l}\text { Optical microscopic } \\
\text { inspection-Lithography }\end{array}$ & Microscope & \\
\hline 28 & $\begin{array}{l}\text { Stripping of Olin PR by } \\
\text { oxygen plasma }\end{array}$ & $\begin{array}{l}\text { Tepla 300E } \\
\text { Barrel Etcher }(2.45 \mathrm{GHz}) \\
\text { - } \mathrm{O}_{2} \text { flow: } 200 \mathrm{sccm}(50 \%) \\
\text { - Power: } 500 \mathrm{~W} \\
\text { - Pressure: } 1.2 \mathrm{mbar} \\
\text { - Time: } 120 \mathrm{sec} .\end{array}$ & $\begin{array}{l}\text { To remove the } \\
\text { fluorocarbon } \\
\text { contamination } \\
\text { produced in step } 26\end{array}$ \\
\hline 29 & $\begin{array}{l}\text { Stripping of polymers in } \\
\mathrm{HNO}_{3} \text { multipurpose }\end{array}$ & $\begin{array}{l}\mathrm{HNO}_{3}(99 \%) \text { Selectipur: MERCK } \\
\text { - Beaker } 1: \mathrm{HNO}_{3}(99 \%) \\
\text { - Time : } 20 \mathrm{~min} \\
\text { - Quick Dump Rinse }<0.1 \mu \mathrm{S} \\
\text { - Spin drying }\end{array}$ & (See Figure 3.6 f) \\
\hline 30 & $\begin{array}{l}\text { Optical microscopic } \\
\text { inspection-Lithography }\end{array}$ & Microscope & \\
\hline 31 & $\begin{array}{l}\text { Sacrificial layer etching } \\
\text { and freeze drying. }\end{array}$ & $\begin{array}{l}\text { BHF(NH4F:HF = 7:1): } \\
\text { VLSI-Etch mixture AF,87.5-12.5 } \\
\text { - Time : } 52 \text { min (including overetching) }\end{array}$ & $\begin{array}{l}\text { See Appendix I for a } \\
\text { detailed procedure of } \\
\text { freeze drying }\end{array}$ \\
\hline 32 & $\begin{array}{l}\text { Optical microscopic } \\
\text { inspection }\end{array}$ & Microscope & $\begin{array}{l}\text { Final inspection of the } \\
\text { devices } \\
\text { (See Figure } 3.6 \mathrm{~g} \text { ) }\end{array}$ \\
\hline
\end{tabular}


Table II. The process document for micro-cantilever integration on micro-ring resonator (chapter 4)

\begin{tabular}{|c|c|c|c|}
\hline Step & Process & Parameters & Remarks \\
\hline 01 & $\begin{array}{l}\text { Dicing of the } 200 \mathrm{~mm} \\
\text { SOI wafer on which the } \\
\text { optical devices are } \\
\text { fabricated }\end{array}$ & & $\begin{array}{l}\text { Diced into } 4 \text { pieces } \\
\text { which fit into the } 100 \\
\text { mm wafer system of } \\
\text { MESA }^{+} \text {Cleanroom }\end{array}$ \\
\hline 02 & Cleaning Standard & $\begin{array}{l}\mathrm{HNO}_{3}(99 \%) \text { Selectipur: MERCK } \\
\mathrm{HNO}_{3}(69 \%) \text { VLSI: } \mathrm{MERCK} \\
\text { - Beaker 1: fumic } \mathrm{HNO}_{3}(99 \%), 5 \mathrm{~min} \\
\text { - Beaker 2: fumic } \mathrm{HNO}_{3}(99 \%), 5 \mathrm{~min} \\
\text { - Quick Dump Rinse }<0.1 \mu \mathrm{S} \\
\text { - Beaker 3: boiling }\left(95^{\circ} \mathrm{C}\right) \mathrm{HNO}_{3}(69 \%), 10 \\
\text { min } \\
\text { - Quick Dump Rinse }<0.1 \mu \mathrm{S} \\
\text { - Spin drying }\end{array}$ & \\
\hline 03 & $\begin{array}{l}\text { LPCVD SiN } \\
\text { stress }\end{array}$ & $\begin{array}{l}\text { Tempress LPCVD/HC } \\
\text { Tube: } \mathrm{G} 3 \\
\text { - } \mathrm{SiH}_{2} \mathrm{Cl}_{2} \text { flow: } 70 \mathrm{sccm} \\
\text { - } \mathrm{NH}_{3} \text { flow: } 18 \mathrm{sccm} \\
\text { - Temperature: } 830^{\circ} \mathrm{C} \\
\text { - Pressure: } 200 \mathrm{mTorr} \\
\text { - Program: SiRN01 } \\
\text { Deposition rate: } 3.9 \sim 5.3 \mathrm{~nm} / \mathrm{min} \\
\mathrm{N}_{\mathrm{f}} \cdot 2.14\end{array}$ & $\begin{array}{l}\text { Thickness } \sim 40 \mathrm{~nm} \\
\text { Time } \sim 00: 10 \text { hr:min } \\
\text { (keep two slot gap in } \\
\text { the boat between } \\
\text { adjacent wafers) } \\
\text { Use at least } 5 \text { dummy } \\
\text { wafers. }\end{array}$ \\
\hline 04 & $\begin{array}{l}\text { Ellipsometer } \\
\text { Measurement }\end{array}$ & Metrology Woollam ellipsometer & $\begin{array}{l}\text { For measuring layer } \\
\text { thickness }\end{array}$ \\
\hline 05 & $\begin{array}{l}\text { Lithography - Priming } \\
\text { (liquid) }\end{array}$ & $\begin{array}{l}\text { HexaMethylDiSilazane (HMDS) } \\
\text { - Dehydration bake }\left(120^{\circ} \mathrm{C}\right): 5 \mathrm{~min} \\
\text { - Spin program: } 5(5000 \mathrm{rpm}, 20 \mathrm{sec})\end{array}$ & \\
\hline 06 & $\begin{array}{l}\text { Lithography - Coating } \\
\text { Olin907-17 }\end{array}$ & $\begin{array}{l}\text { - Spinning acceleration: } 4000 \mathrm{rpm} / \mathrm{s} \\
\text { - Spinning speed: } 4000 \mathrm{rpm} \\
\text { - Spinning time: } 20 \mathrm{~s} \\
\text { - Prebake }\left(95^{\circ} \mathrm{C}\right): 90 \mathrm{~s}\end{array}$ & $\begin{array}{l}\text { To protect the front } \\
\text { side of the wafer during } \\
\text { backside etching in step } \\
08 \text {. }\end{array}$ \\
\hline 07 & Plasma cleaning (Etske) & $\begin{array}{l}\text { Elektrotech PF310/340 } \\
\text { Dirty chamber-Styros electrode } \\
\text { - Electrode temp.: } 10{ }^{\circ} \mathrm{C} \\
\text { - } \mathrm{O}_{2} \text { flow: } 20 \mathrm{sccm} \\
\text { - Pressure: } 0 \mathrm{mTorr} \\
\text { - Power: } 150 \mathrm{~W}\end{array}$ & $\begin{array}{l}\text { Removal of Residues } \\
\text { Time: 10:00 min:sec }\end{array}$ \\
\hline 08 & $\begin{array}{l}\text { Plasma etching } \operatorname{SiN}_{x} \\
\text { (Etske) }\end{array}$ & $\begin{array}{l}\text { Elektrotech PF310/340 } \\
\text { Dirty chamber } \\
\text { Styros electrode } \\
\text { - Electrode temp.: } 10{ }^{\circ} \mathrm{C} \\
\text { - } \mathrm{CHF}_{3} \text { flow: } 25 \mathrm{sccm} \\
\text { - } \mathrm{O}_{2} \text { flow: } 5 \mathrm{sccm} \\
\text { - Pressure: } 10 \mathrm{~m} \text { Torr } \\
\text { - Power: } 75 \mathrm{~W}\end{array}$ & $\begin{array}{l}\text { Backside } \\
\text { Etch rate: } 55 \mathrm{~nm} / \mathrm{min} \\
\text { Time: } 2 \mathrm{~min}\end{array}$ \\
\hline
\end{tabular}




\begin{tabular}{|c|c|c|c|}
\hline & & $\begin{array}{l}\text { Etchrate } \mathrm{SiN}_{\mathrm{x}}=55 \mathrm{~nm} / \mathrm{min}\left(\text { for } \mathrm{V}_{\mathrm{DC}}=-460 \mathrm{~V}\right) \\
\text { Etchrate Olin resist }=95 \mathrm{~nm} / \mathrm{min} \\
\text { If DC-Bias }<375 \mathrm{~V} \text { apply chamber clean }\end{array}$ & \\
\hline 09 & $\begin{array}{l}\text { Stripping of polymers in } \\
\mathrm{HNO}_{3} \text { multipurpose }\end{array}$ & $\begin{array}{l}\mathrm{HNO}_{3}(99 \%) \text { Selectipur: MERCK } \\
\text { - Beaker } 1: \mathrm{HNO}_{3}\left(99^{\circ} \%\right) \\
\text { - Time : } 20 \mathrm{~min} \\
\text { - Quick Dump Rinse <0.1 } \mathrm{\mu S} \\
\text { - Spin drying }\end{array}$ & \\
\hline 10 & Cleaning Standard & $\begin{array}{l}\mathrm{HNO}_{3}(99 \%) \text { Selectipur: MERCK } \\
\mathrm{HNO}_{3}(69 \%) \text { VLSI: MERCK } \\
\text { - Beaker 1: fumic } \mathrm{HNO}_{3}(99 \%), 5 \mathrm{~min} \\
\text { - Beaker 2: fumic } \mathrm{HNO}_{3}(99 \%), 5 \mathrm{~min} \\
\text { - Quick Dump Rinse }<0.1 \mu \mathrm{S} \\
\text { - Beaker 3: boiling }\left(95^{\circ} \mathrm{C}\right) \mathrm{HNO}_{3}(69 \%), 10 \\
\text { min } \\
\text { - Quick Dump Rinse <0.1 } \mu \mathrm{S} \\
\text { - Spin drying }\end{array}$ & \\
\hline 11 & LPCVD TEOS & $\begin{array}{l}\text { Tempress LPCVD B4 } \\
\text { Tube: B4 TEOS } \\
\text { - Bubbler: } 40^{\circ} \mathrm{C} \\
\text { - } \mathrm{N}_{2} \text { flow: } 250 \mathrm{sccm} \\
\text { - Temperature: } 700{ }^{\circ} \mathrm{C} \\
\text { - Pressure: } 150 \mathrm{~m} \text { Torr } \\
\text { - Program: TEOS } 05 \\
\text { Deposition rate: } 10.7 \mathrm{~nm} / \mathrm{min} \\
\mathrm{N}_{\mathrm{f}}: 1.44\end{array}$ & $\begin{array}{l}\text { Thickness } \sim 200 \mathrm{~nm} \\
\text { Time } \sim 00: 21 \mathrm{hr}: \mathrm{min} \\
\text { Use at least } 6 \text { dummy } \\
\text { wafers }\end{array}$ \\
\hline 12 & $\begin{array}{l}\text { Ellipsometer } \\
\text { Measurement }\end{array}$ & Metrology Woollam ellipsometer & $\begin{array}{l}\text { For measuring layer } \\
\text { thickness on dummy } \\
\text { silicon wafer }\end{array}$ \\
\hline 13 & $\begin{array}{l}\text { Wafer curvature } \\
\text { measurement }\end{array}$ & $\begin{array}{l}\text { Veeco Dektak } 8 \\
\text { To measure the stress in the deposited } \operatorname{SiN}_{\mathrm{x}} \\
\text { layer }\end{array}$ & $\begin{array}{l}\text { Measure on dummy } \\
\text { silicon wafers at } 0^{\circ}, 45^{\circ} \text {, } \\
90^{\circ} \text { and at } 135^{\circ} \text { and use } \\
\text { them in steps } 14,15 \text { and } \\
17 \mathrm{~A} \text {. }\end{array}$ \\
\hline 14 & Cleaning Standard & $\begin{array}{l}\mathrm{HNO}_{3}(99 \%) \text { Selectipur: MERCK } \\
\mathrm{HNO}_{3}(69 \%) \text { VLSI: } \mathrm{MERCK} \\
\text { - Beaker 1: fumic } \mathrm{HNO}_{3}(99 \%), 5 \mathrm{~min} \\
\text { - Beaker 2: fumic } \mathrm{HNO}_{3}(99 \%), 5 \mathrm{~min} \\
\text { - Quick Dump Rinse }<0.1 \mu \mathrm{S} \\
\text { - Beaker 3: boiling }\left(95^{\circ} \mathrm{C}\right) \mathrm{HNO}_{3}(69 \%), 10 \\
\text { min } \\
\text { - Quick Dump Rinse }<0.1 \mu \mathrm{S} \\
\text { - Spin drying }\end{array}$ & \\
\hline 15 & $\begin{array}{l}\text { LPCVD SiN } \text { - low }_{\text {stress }}\end{array}$ & $\begin{array}{l}\text { Tempress LPCVD/HC } \\
\text { Tube: G3 } \\
\text { - } \mathrm{SiH}_{2} \mathrm{Cl}_{2} \text { flow: } 70 \mathrm{sccm} \\
\text { - } \mathrm{NH}_{3} \text { flow: } 18 \mathrm{sccm} \\
\text { - Temperature: } 830{ }^{\circ} \mathrm{C} \\
\text { - Pressure: } 200 \mathrm{~m} \text { Torr } \\
\text { - Program: SiRN01 }\end{array}$ & $\begin{array}{l}\text { Thickness } \sim 1.0 \mu \mathrm{m} \\
\text { Time } \sim 03.55 \mathrm{hr}: \mathrm{min} \\
\text { Use at least } 6 \text { dummy } \\
\text { wafers }\end{array}$ \\
\hline
\end{tabular}




\begin{tabular}{|c|c|c|c|}
\hline & & $\begin{array}{l}\text { Deposition rate: } 3.9 \sim 5.3 \mathrm{~nm} / \mathrm{min} \\
\mathrm{N}_{\mathrm{f}}: 2.14\end{array}$ & \\
\hline 16 & $\begin{array}{l}\text { Ellipsometer } \\
\text { Measurement }\end{array}$ & Metrology Woollam ellipsometer & $\begin{array}{l}\text { For measuring layer } \\
\text { thickness on dummy } \\
\text { silicon wafer }\end{array}$ \\
\hline 17 & $\begin{array}{l}\text { Wafer curvature } \\
\text { measurement }\end{array}$ & $\begin{array}{l}\text { Veeco Dektak } 8 \\
\text { (A) For } \mathrm{SiN}_{\mathbf{x}} \text { layer: Use the silicon wafers } \\
\text { measured in step 13. Etch the } \mathrm{SiN}_{\mathrm{x}} \text { on the back } \\
\text { side using step 08. And measure the final wafer } \\
\text { curvature. } \\
\text { (B) For } \mathbf{A u}+\mathbf{C r} \text { layer: Use new silicon dummy } \\
\text { wafers and measure the initial wafer curvature. }\end{array}$ & $\begin{array}{l}\text { Measure the wafer } \\
\text { curvature at } 0^{\circ}, 45^{\circ}, 90^{\circ} \\
\text { and at } 135^{\circ} .\end{array}$ \\
\hline 18 & Cleaning Standard & $\begin{array}{l}\mathrm{HNO}_{3}(99 \%) \text { Selectipur: MERCK } \\
\mathrm{HNO}_{3}(69 \%) \text { VLSI: MERCK } \\
\text { - Beaker 1: fumic } \mathrm{HNO}_{3}(99 \%), 5 \mathrm{~min} \\
\text { - Beaker 2: fumic } \mathrm{HNO}_{3}(99 \%), 5 \mathrm{~min} \\
\text { - Quick Dump Rinse }<0.1 \mu \mathrm{S} \\
\text { - Beaker 3: boiling }\left(95{ }^{\circ} \mathrm{C}\right) \mathrm{HNO}_{3}(69 \%), 10 \\
\text { min } \\
\text { - Quick Dump Rinse }<0.1 \mu \mathrm{S} \\
\text { - Spin drying }\end{array}$ & \\
\hline 19 & Sputtering of $\mathrm{Cr}$ & $\begin{array}{l}\text { Sputterke } \\
\text { - Electrode temperature : Water cooled } \\
\text { electrode } \\
\text { - Power: } 100 \mathrm{~W} \text { (to reduce the stress and } \\
\text { thickness) } \\
\text { - Base Pressure: }<1 \mathrm{X} 10^{-6} \mathrm{mbar} \\
\text { - Sputter Pressure: }<6.6 \times 10^{-3} \mathrm{mbar} \\
\text { Deposition rate }(100 \mathrm{~mm} \text { wafer): } 15 \mathrm{~nm} / \mathrm{min}\end{array}$ & $\begin{array}{l}\text { Thickness: } 8 \mathrm{~nm} \\
\text { Pre-Time: 01:00 min } \\
\text { Sputter time : 01:00 min } \\
\text { * Sputter also on } \\
\text { dummy wafers with a } \\
\text { scotch tape on it to later } \\
\text { measure the layer } \\
\text { thickness by Dektak. }\end{array}$ \\
\hline 20 & Sputtering of $\mathrm{Au}$ & $\begin{array}{l}\text { Sputterke } \\
\text { - Electrode temperature : Water cooled } \\
\text { electrode } \\
\text { - Power: } 200 \mathrm{~W} \\
\text { - Base Pressure: }<1 \times 10^{-6} \mathrm{mbar} \\
\text { - Sputter Pressure: }<6.6 \text { X } 10^{-3} \mathrm{mbar} \\
\text { Deposition rate }(100 \mathrm{~mm} \text { wafer }): 45-50 \\
\mathrm{~nm} / \mathrm{min}\end{array}$ & $\begin{array}{l}\text { Thickness: } 50 \mathrm{~nm} \\
\text { Pre-Time: 01:00 min } \\
\text { Sputter time : 01:07 min } \\
\text { * Sputter also on } \\
\text { dummy wafers with } \\
\text { another scotch tape on } \\
\text { it to later measure the } \\
\text { layer thickness by } \\
\text { Dektak. }\end{array}$ \\
\hline 21 & $\begin{array}{l}\text { Wafer curvature } \\
\text { measurement }\end{array}$ & $\begin{array}{l}\text { Veeco Dektak } 8 \\
\text { Use the silicon dummy wafers used in step } 17 \\
B \text {. }\end{array}$ & $\begin{array}{l}\text { Measure at } 0^{\circ}, 45^{\circ}, 90^{\circ} \\
\text { and at } 135^{\circ} .\end{array}$ \\
\hline 22 & $\begin{array}{l}\text { Layer Thickness } \\
\text { Measurement }\end{array}$ & Veeco Dektak 8 & $\begin{array}{l}\text { Remove the scotch tape } \\
\text { from dummy wafers } \\
\text { and measure the step } \\
\text { height of } \mathrm{Au} \text { and } \mathrm{Cr} \\
\text { layers. }\end{array}$ \\
\hline
\end{tabular}




\begin{tabular}{|c|c|c|c|}
\hline 23 & $\begin{array}{l}\text { Lithography - Priming } \\
\text { (liquid) }\end{array}$ & $\begin{array}{l}\text { HexaMethylDiSilazane (HMDS) } \\
\text { - Dehydration bake }\left(120^{\circ} \mathrm{C}\right): 5 \mathrm{~min} \\
\text { - Spin program: } 5(5000 \mathrm{rpm}, 20 \mathrm{sec})\end{array}$ & \\
\hline 24 & $\begin{array}{l}\text { Lithography - Coating } \\
\text { Olin907-17 }\end{array}$ & $\begin{array}{l}\text { - Spinning acceleration: } 4000 \mathrm{rpm} / \mathrm{s} \\
\text { - Spinning speed: } 4000 \mathrm{rpm} \\
\text { - Spinning time: } 20 \mathrm{~s} \\
\text { - Prebake }\left(95^{\circ} \mathrm{C}\right): 90 \mathrm{~s}\end{array}$ & $\begin{array}{l}\text { Wait } 30 \text { min before } \\
\text { exposure to avoid } \\
\text { stiction }\end{array}$ \\
\hline 25 & $\begin{array}{l}\text { Lithography - } \\
\text { Alignment \& Exposure } \\
\text { Olin } 907-17\end{array}$ & $\begin{array}{l}\text { Electronic Vision Group } 20 \text { Mask Aligner } \\
\text { - Hg-lamp: } 12 \mathrm{~mW} / \mathrm{cm}^{2} \\
\text { - Exposure Time: } 4.1 \mathrm{sec}\end{array}$ & $\begin{array}{l}\text { Mask: For patterning } \\
\text { the metal layers } \\
\text { Hard contact }\end{array}$ \\
\hline 26 & $\begin{array}{l}\text { Lithography - } \\
\text { Development Olin } \\
\text { Resist }\end{array}$ & $\begin{array}{l}\text { Developer: OPD } 4262 \\
\text { - After Exposure Bake }\left(120^{\circ} \mathrm{C}\right): 60 \mathrm{sec} \\
\text { Development: } \\
\text { - Time: } 30 \mathrm{sec} \text { in Beaker } 1 \\
\text { - Time: } 15-30 \mathrm{sec} \text { in Beaker } 2 \\
\text { - Quick Dump Rinse <0.1 } \mu \mathrm{S} \\
\text { - Spin drying }\end{array}$ & \\
\hline 27 & $\begin{array}{l}\text { Optical microscopic } \\
\text { inspection-Lithography }\end{array}$ & Microscope & \\
\hline 28 & $\begin{array}{l}\text { Lithography - Postbake } \\
\text { standard }\end{array}$ & Hot plate at $120^{\circ} \mathrm{C}: 30 \mathrm{~min}$ & \\
\hline 29 & $\begin{array}{l}\text { Ozone anneal of Olin } \\
907-17\end{array}$ & $\begin{array}{l}\text { UV PRS-100 } \\
\text { - To improve wetting during etching } \\
\text { - Time: } 300 \mathrm{sec}\end{array}$ & \\
\hline 30 & Wet etching of $\mathrm{Au}$ & $\begin{array}{l}\text { Gold etchant: }\left(\mathrm{KI}=132 \mathrm{~g}, \mathrm{I}_{2}=18 \mathrm{~g}, \mathrm{DI}=\right. \\
1200 \mathrm{ml} \text {, in which glycerin }(600 \mathrm{ml}) \text { is added to } \\
\text { reduce the excessive undercut }) \\
\text { - Temperature }: 30^{\circ} \mathrm{C} \\
\text { - } \text { Quick Dump Rinse }<0.1 \mu \mathrm{S} \\
\text { - Spin drying } \\
\text { Etch rate }=1 \mathrm{~nm} / \mathrm{min}\end{array}$ & Time $=50 \mathrm{sec}$ \\
\hline 31 & Wet etching of $\mathrm{Cr}$ & $\begin{array}{l}\text { Chromium etch LSI selectipur : MERCK } \\
111547.2500 \\
\text { - Quick Dump Rinse }<0.1 \mu \mathrm{S} \\
\text { - Spin drying } \\
\text { Etch rate }=0.5 \mathrm{~nm} / \mathrm{min}\end{array}$ & $\begin{array}{l}\text { Thickness: } 8 \mathrm{~nm} \\
\text { Time:00:16 sec }\end{array}$ \\
\hline 32 & $\begin{array}{l}\text { Optical microscopic } \\
\text { inspection-Lithography }\end{array}$ & Microscope & \\
\hline 33 & $\begin{array}{l}\text { Stripping of polymers in } \\
\mathrm{HNO}_{3} \text { multipurpose }\end{array}$ & $\begin{array}{l}\mathrm{HNO}_{3}(99 \%) \text { Selectipur: MERCK } \\
\text { - Beaker } 1: \mathrm{HNO}_{3}(99 \%) \\
\text { - Time : } 20 \mathrm{~min} \\
\text { - Quick Dump Rinse }<0.1 \mu \mathrm{S} \\
\text { - Spin drying }\end{array}$ & \\
\hline 34 & Lithography - Priming & $\begin{array}{l}\text { HexaMethylDiSilazane (HMDS) } \\
\text { - Dehydration bake }\left(120^{\circ} \mathrm{C}\right): 5 \mathrm{~min}\end{array}$ & \\
\hline
\end{tabular}




\begin{tabular}{|c|c|c|c|}
\hline & (liquid) & - Spin program: 5 (5000 rpm, $20 \mathrm{sec})$ & \\
\hline 35 & $\begin{array}{l}\text { Lithography - Coating } \\
\text { Olin907-35 }\end{array}$ & $\begin{array}{l}\text { - Spinning acceleration: } 4000 \mathrm{rpm} / \mathrm{s} \\
\text { - Spinning speed: } 4000 \mathrm{rpm} \\
\text { - Spinning time: } 20 \mathrm{~s} \\
\text { - Prebake }\left(95^{\circ} \mathrm{C}\right): 90 \mathrm{~s}\end{array}$ & $\begin{array}{l}\text { Wait } 30 \text { min before } \\
\text { exposure to avoid } \\
\text { stiction }\end{array}$ \\
\hline 36 & $\begin{array}{l}\text { Lithography - } \\
\text { Alignment \& Exposure } \\
\text { Olin } 907-35\end{array}$ & $\begin{array}{l}\text { Electronic Vision Group } 20 \text { Mask Aligner } \\
\text { - Hg-lamp: } 12 \mathrm{~mW} / \mathrm{cm}^{2} \\
\text { - Exposure Time: } 9.3 \mathrm{sec}\end{array}$ & $\begin{array}{l}\text { Mask: For patterning } \\
\text { SiN }_{x} \text { cantilever layer } \\
\text { Hard contact }\end{array}$ \\
\hline 37 & $\begin{array}{l}\text { Lithography - } \\
\text { Development Olin } \\
\text { Resist }\end{array}$ & $\begin{array}{l}\text { Developer: OPD } 4262 \\
\text { - After Exposure Bake }\left(120^{\circ} \mathrm{C}\right): 60 \mathrm{sec} \\
\text { Development: } \\
\text { - Time: } 30 \mathrm{sec} \text { in Beaker } 1 \\
\text { - Time: } 15-30 \mathrm{sec} \text { in Beaker } 2 \\
\text { - Quick Dump Rinse }<0.1 \mu \mathrm{S} \\
\text { - Spin drying }\end{array}$ & $\begin{array}{l}\text { Wait at least } 30 \mathrm{~min} \\
\text { before post bake }\end{array}$ \\
\hline 38 & $\begin{array}{l}\text { Optical microscopic } \\
\text { inspection-Lithography }\end{array}$ & Microscope & \\
\hline 39 & $\begin{array}{l}\text { Lithography - Postbake } \\
\text { standard }\end{array}$ & Hot plate at $120^{\circ} \mathrm{C}: 30 \mathrm{~min}$ & \\
\hline 40 & $\begin{array}{l}\text { Plasma cleaning (Etske) } \\
\text { Cleaning }\end{array}$ & $\begin{array}{l}\text { Elektrotech PF310/340 } \\
\text { Dirty chamber } \\
\text { Styros electrode } \\
\text { - Electrode temp.: } 10{ }^{\circ} \mathrm{C} \\
\text { - } \mathrm{O}_{2} \text { flow: } 20 \mathrm{sccm} \\
\text { - Pressure: } 0 \mathrm{mTorr} \\
\text { - Power: } 150 \mathrm{~W}\end{array}$ & $\begin{array}{l}\text { Removal of Residues } \\
\text { Time: 10:00 min:sec }\end{array}$ \\
\hline 41 & $\begin{array}{l}\text { Plasma etching } \mathrm{SiN}_{\mathrm{x}} \\
\text { (Etske) }\end{array}$ & $\begin{array}{l}\text { Elektrotech PF310/340 } \\
\text { Dirty chamber } \\
\text { Styros electrode } \\
\text { - Electrode temp.: } 10^{\circ} \mathrm{C} \\
\text { - } \mathrm{CHF}_{3} \text { flow: } 25 \mathrm{sccm} \\
\text { - } \mathrm{O}_{2} \text { flow: } 5 \mathrm{sccm} \\
\text { - Pressure: } 10 \mathrm{mTorr} \\
\text { - Power: } 75 \mathrm{~W} \\
\text { Etchrate } \mathrm{SiN}_{\mathrm{x}}=55 \mathrm{~nm} / \mathrm{min}\left(\text { for } \mathrm{V}_{\mathrm{DC}}=-460 \mathrm{~V}\right) \\
\text { Etchrate Olin resist }=95 \mathrm{~nm} / \mathrm{min} \\
\text { If DC-Bias }<375 \mathrm{~V} \text { apply chamber clean }\end{array}$ & $\begin{array}{l}\text { Backside } \\
\text { Etch rate: } 55 \mathrm{~nm} / \mathrm{min} \\
\text { Time }: 25 \mathrm{~min}\end{array}$ \\
\hline 42 & $\begin{array}{l}\text { Plasma etching } \mathrm{SiN}_{\mathrm{x}} \\
\text { (Etske) }\end{array}$ & $\begin{array}{l}\text { Elektrotech PF310/340 } \\
\text { Dirty chamber } \\
\text { Styros electrode } \\
\text { - Electrode temp.: } 10^{\circ} \mathrm{C} \\
\text { - } \mathrm{CHF}_{3} \text { flow: } 25 \mathrm{sccm} \\
\text { - } \mathrm{O}_{2} \text { flow: } 5 \mathrm{sccm} \\
\text { - Pressure: } 10 \mathrm{mTorr} \\
\text { - Power: } 75 \mathrm{~W} \\
\text { Etchrate } \mathrm{SiN}_{\mathrm{x}}=50 \mathrm{~nm} / \mathrm{min}\left(\text { for } \mathrm{V}_{\mathrm{DC}}=-460 \mathrm{~V}\right) \\
\text { Etchrate Olin resist }=95 \mathrm{~nm} / \mathrm{min}\end{array}$ & $\begin{array}{l}\text { Frontside } \\
\text { Etch rate: } 55 \mathrm{~nm} / \mathrm{min} \\
\text { Check the exact } \\
\text { thickness of deposited } \\
\text { SiN }_{\mathrm{x}} \text { layer with dummy } \\
\text { wafers and overetch } \\
\text { considering the } \\
\text { sacrificial layer } \\
\text { thickness. }\end{array}$ \\
\hline
\end{tabular}




\begin{tabular}{|c|c|c|c|}
\hline & & If DC-Bias $<375 \mathrm{~V}$ apply chamber clean & Etch time $\sim 20 \mathrm{~min}$ \\
\hline 43 & $\begin{array}{l}\text { Optical microscopic } \\
\text { inspection-Lithography }\end{array}$ & Microscope & \\
\hline 44 & $\begin{array}{l}\text { Stripping of Olin PR by } \\
\text { oxygen plasma }\end{array}$ & $\begin{array}{l}\text { Tepla 300E } \\
\text { Barrel Etcher }(2.45 \mathrm{GHz}) \\
\text { - } \mathrm{O}_{2} \text { flow: } 200 \mathrm{sccm}(50 \%) \\
\text { - Power: } 500 \mathrm{~W} \\
\text { - Pressure: } 1.2 \mathrm{mbar} \\
\text { - Time: } 120 \mathrm{sec} .\end{array}$ & $\begin{array}{l}\text { To remove the } \\
\text { fluorocarbon } \\
\text { contamination } \\
\text { produced in step } 41\end{array}$ \\
\hline 45 & $\begin{array}{l}\text { Optical microscopic } \\
\text { inspection-Lithography }\end{array}$ & Microscope & \\
\hline 46 & $\begin{array}{l}\text { Stripping of polymers in } \\
\mathrm{HNO}_{3} \text { multipurpose }\end{array}$ & $\begin{array}{l}\mathrm{HNO}_{3}(99 \%) \text { Selectipur: MERCK } \\
\text { - Beaker } 1: \mathrm{HNO}_{3}(99 \%) \\
\text { - Time : } 20 \mathrm{~min} \\
\text { - Quick Dump Rinse <0.1 } \mathrm{SS} \\
\text { - Spin drying }\end{array}$ & \\
\hline 47 & $\begin{array}{l}\text { Optical microscopic } \\
\text { inspection-Lithography }\end{array}$ & Microscope & \\
\hline 48 & $\begin{array}{l}\text { Sacrificial layer etching } \\
\text { and freeze drying. }\end{array}$ & $\begin{array}{l}\mathrm{BHF}\left(\mathrm{NH}_{4} \mathrm{~F}: \mathrm{HF}=7: 1\right): \\
\text { VLSI-Etch mixture } \mathrm{AF}, 87.5-12.5 \\
\text { - Time }: 52 \text { min (including overetching) }\end{array}$ & $\begin{array}{l}\text { See Appendix I for a } \\
\text { detailed procedure of } \\
\text { freeze drying }\end{array}$ \\
\hline 49 & $\begin{array}{l}\text { Optical microscopic } \\
\text { inspection }\end{array}$ & Microscope & $\begin{array}{l}\text { Final inspection of the } \\
\text { devices }\end{array}$ \\
\hline
\end{tabular}


Table III. The process document for integrating micro-cantilevers with photonic crystals (chapter 6)

\begin{tabular}{|c|c|c|c|}
\hline Step & Process & Parameters & Remarks \\
\hline 01 & $\begin{array}{l}\text { Dicing of the } 200 \mathrm{~mm} \\
\text { SOI wafer on which the } \\
\text { optical devices are } \\
\text { fabricated }\end{array}$ & & $\begin{array}{l}\text { Diced into } 4 \text { pieces } \\
\text { which fit into the } 100 \\
\text { mm wafer system of } \\
\text { MESA }^{+} \text {Cleanroom }\end{array}$ \\
\hline 02 & Cleaning Standard & $\begin{array}{l}\mathrm{HNO}_{3}(99 \%) \text { Selectipur: MERCK } \\
\mathrm{HNO}_{3}(69 \%) \text { VLSI: } \mathrm{MERCK} \\
\text { - Beaker 1: fumic } \mathrm{HNO}_{3}(99 \%), 5 \mathrm{~min} \\
\text { - Beaker 2: fumic } \mathrm{HNO}_{3}(99 \%), 5 \mathrm{~min} \\
\text { - Quick Dump Rinse <0.1 } \mu \mathrm{S} \\
\text { - Beaker 3: boiling }\left(95^{\circ} \mathrm{C}\right) \mathrm{HNO}_{3}(69 \%), 10 \\
\text { min } \\
\text { - Quick Dump Rinse <0.1 } \mathrm{SS} \\
\text { - Spin drying }\end{array}$ & \\
\hline 03 & $\begin{array}{l}\text { LPCVD SiN } \\
\text { stress }\end{array}$ & $\begin{array}{l}\text { Tempress LPCVD/HC } \\
\text { Tube: G3 } \\
\text { - } \mathrm{SiH}_{2} \mathrm{Cl}_{2} \text { flow: } 70 \mathrm{sccm} \\
\text { - } \mathrm{NH}_{3} \text { flow: } 18 \mathrm{sccm} \\
\text { - Temperature: } 830{ }^{\circ} \mathrm{C} \\
\text { - Pressure: } 200 \mathrm{~m} \text { Torr } \\
\text { - Program: SiRN01 } \\
\text { Deposition rate: } 3.9 \sim 5.3 \mathrm{~nm} / \mathrm{min} \\
\mathrm{N}_{\mathrm{f}} \cdot 2.14\end{array}$ & $\begin{array}{l}\text { Thickness } \sim 40 \mathrm{~nm} \\
\text { Time } \sim 00: 10 \mathrm{hr}: \mathrm{min} \\
\text { (keep two slot gap in } \\
\text { the boat between } \\
\text { adjacent wafers) } \\
\text { Use at least } 4 \text { silicon } \\
\text { dummy wafers. }\end{array}$ \\
\hline 04 & $\begin{array}{l}\text { Ellipsometer } \\
\text { Measurement }\end{array}$ & Metrology Woollam ellipsometer & $\begin{array}{l}\text { For measuring layer } \\
\text { thickness }\end{array}$ \\
\hline 05 & $\begin{array}{l}\text { Lithography - Priming } \\
\text { (liquid) }\end{array}$ & $\begin{array}{l}\text { HexaMethylDiSilazane (HMDS) } \\
\text { - Dehydration bake }\left(120{ }^{\circ} \mathrm{C}\right): 5 \mathrm{~min} \\
\text { - Spin program: } 5(5000 \mathrm{rpm}, 20 \mathrm{sec})\end{array}$ & \\
\hline 06 & $\begin{array}{l}\text { Lithography - Coating } \\
\text { Olin907-17 }\end{array}$ & $\begin{array}{l}\text { - Spinning acceleration: } 4000 \mathrm{rpm} / \mathrm{s} \\
\text { - Spinning speed: } 4000 \mathrm{rpm} \\
\text { - Spinning time: } 20 \mathrm{~s} \\
\text { - Prebake }\left(95^{\circ} \mathrm{C}\right): 90 \mathrm{~s}\end{array}$ & $\begin{array}{l}\text { To protect the front } \\
\text { side of the wafer during } \\
\text { backside etching in step } \\
08 .\end{array}$ \\
\hline 07 & Plasma cleaning (Etske) & $\begin{array}{l}\text { Elektrotech PF310/340 } \\
\text { Dirty chamber } \\
\text { Styros electrode } \\
\text { - Electrode temp.: } 10{ }^{\circ} \mathrm{C} \\
\text { - } \mathrm{O}_{2} \text { flow: } 20 \mathrm{sccm} \\
\text { - Pressure: } 0 \mathrm{mTorr} \\
\text { - Power: } 150 \mathrm{~W}\end{array}$ & $\begin{array}{l}\text { Removal of Residues } \\
\text { Time: 10:00 min:sec }\end{array}$ \\
\hline 08 & $\begin{array}{l}\text { Plasma etching } \mathrm{SiN}_{\mathrm{x}} \\
\text { (Etske) }\end{array}$ & $\begin{array}{l}\text { Elektrotech PF310/340 } \\
\text { Dirty chamber } \\
\text { Styros electrode } \\
\text { - Electrode temp.: } 10{ }^{\circ} \mathrm{C} \\
\text { - } \mathrm{CHF}_{3} \text { flow: } 25 \mathrm{sccm}\end{array}$ & $\begin{array}{l}\text { Backside } \\
\text { Time: } 2 \text { min }\end{array}$ \\
\hline
\end{tabular}




\begin{tabular}{|c|c|c|c|}
\hline & & $\begin{array}{l}\text { - } \mathrm{O}_{2} \text { flow: } 5 \mathrm{sccm} \\
\text { - Pressure: } 10 \mathrm{~m} \text { Torr } \\
\text { - Power: } 75 \mathrm{~W} \\
\text { Etchrate } \mathrm{SiN}_{\mathrm{x}}=55 \mathrm{~nm} / \mathrm{min}\left(\text { for } \mathrm{V}_{\mathrm{DC}}=-460 \mathrm{~V}\right) \\
\text { Etchrate Olin resist }=95 \mathrm{~nm} / \mathrm{min} \\
\text { If DC-Bias }<375 \mathrm{~V} \text { apply chamber clean }\end{array}$ & \\
\hline 09 & $\begin{array}{l}\text { Stripping of polymers in } \\
\mathrm{HNO}_{3} \text { multipurpose }\end{array}$ & $\begin{array}{l}\mathrm{HNO}_{3}(99 \%) \text { Selectipur: MERCK } \\
\text { - Beaker } 1: \mathrm{HNO}_{3}(99 \%) \\
\text { - Time : } 20 \mathrm{~min} \\
\text { - Quick Dump Rinse <0.1 } \mathrm{SS} \\
\text { - Spin drying }\end{array}$ & \\
\hline 10 & Cleaning Standard & $\begin{array}{l}\mathrm{HNO}_{3}(99 \%) \text { Selectipur: MERCK } \\
\mathrm{HNO}_{3}(69 \%) \text { VLSI: MERCK } \\
\text { - Beaker 1: fumic } \mathrm{HNO}_{3}(99 \%), 5 \mathrm{~min} \\
\text { - Beaker 2: fumic } \mathrm{HNO}_{3}(99 \%), 5 \mathrm{~min} \\
\text { - Quick Dump Rinse <0.1 } \mu \mathrm{S} \\
\text { - Beaker 3: boiling }\left(95^{\circ} \mathrm{C}\right) \mathrm{HNO}_{3}(69 \%), 10 \\
\text { min } \\
\text { - Quick Dump Rinse <0.1 } \mu \mathrm{S} \\
\text { - Spin drying }\end{array}$ & \\
\hline 11 & LPCVD TEOS & $\begin{array}{l}\text { Tempress LPCVD B4 } \\
\text { Tube: B4 TEOS } \\
\text { - Bubbler: } 40^{\circ} \mathrm{C} \\
\text { - } \mathrm{N}_{2} \text { flow: } 250 \mathrm{sccm} \\
\text { - Temperature: } 700{ }^{\circ} \mathrm{C} \\
\text { - Pressure: } 150 \mathrm{~m} \text { Torr } \\
\text { - Program: TEOS } 05 \\
\text { Deposition rate: } 10.7 \mathrm{~nm} / \mathrm{min} \\
\mathrm{N}_{\mathrm{f}}: 1.44\end{array}$ & $\begin{array}{l}\text { Thickness } \sim 90 \mathrm{~nm} \\
\text { Time } \sim 00: 10 \mathrm{hr}: \mathrm{min} \\
\text { Use at least } 6 \text { dummy } \\
\text { wafers }\end{array}$ \\
\hline 12 & $\begin{array}{l}\text { Ellipsometer } \\
\text { Measurement }\end{array}$ & Metrology Woollam ellipsometer & $\begin{array}{l}\text { For measuring layer } \\
\text { thickness on silicon } \\
\text { dummy wafer }\end{array}$ \\
\hline 13 & $\begin{array}{l}\text { Lithography - Priming } \\
\text { (liquid) }\end{array}$ & $\begin{array}{l}\text { HexaMethylDiSilazane (HMDS) } \\
\text { - Dehydration bake }\left(120^{\circ} \mathrm{C}\right): 5 \mathrm{~min} \\
\text { - Spin program: } 5(5000 \mathrm{rpm}, 20 \mathrm{sec})\end{array}$ & \\
\hline 14 & $\begin{array}{l}\text { Lithography - Coating } \\
\text { Olin907-17 }\end{array}$ & $\begin{array}{l}\text { - Spinning acceleration: } 4000 \mathrm{rpm} / \mathrm{s} \\
\text { - Spinning speed: } 4000 \mathrm{rpm} \\
\text { - Spinning time: } 20 \mathrm{~s} \\
\text { - Prebake }\left(95^{\circ} \mathrm{C}\right): 90 \mathrm{~s}\end{array}$ & $\begin{array}{l}\text { Wait } 30 \text { min before } \\
\text { exposure to avoid } \\
\text { stiction }\end{array}$ \\
\hline 15 & $\begin{array}{l}\text { Lithography - } \\
\text { Alignment \& Exposure } \\
\text { Olin } 907-17\end{array}$ & $\begin{array}{l}\text { Electronic Vision Group } 20 \text { Mask Aligner } \\
\text { - Hg-lamp: } 12 \mathrm{~mW} / \mathrm{cm}^{2} \\
\text { - Exposure Time: } 4.1 \mathrm{sec}\end{array}$ & $\begin{array}{l}\text { Mask: Etch masks } \\
\text { Hard contact }\end{array}$ \\
\hline 16 & $\begin{array}{l}\text { Lithography - } \\
\text { Development Olin } \\
\text { Resist }\end{array}$ & $\begin{array}{l}\text { Developer: OPD4262 } \\
\text { - After Exposure Bake }\left(120{ }^{\circ} \mathrm{C}\right): 60 \mathrm{sec} \\
\text { Development: } \\
\text { - Time: } 30 \mathrm{sec} \text { in Beaker } 1 \\
\text { - Time: } 15-30 \mathrm{sec} \text { in Beaker } 2 \\
\text { - Quick Dump Rinse }<0.1 \mu \mathrm{S}\end{array}$ & \\
\hline
\end{tabular}




\begin{tabular}{|c|c|c|c|}
\hline & & - Spin drying & \\
\hline 17 & $\begin{array}{l}\text { Optical microscopic } \\
\text { inspection-Lithography }\end{array}$ & Microscope & \\
\hline 18 & $\begin{array}{l}\text { Lithography - Postbake } \\
\text { standard }\end{array}$ & Hot plate at $120^{\circ} \mathrm{C}: 30 \mathrm{~min}$ & \\
\hline 19 & $\begin{array}{l}\text { Ozone anneal of Olin } \\
907-17\end{array}$ & $\begin{array}{l}\text { UV PRS-100 } \\
\text { - To improve wetting during etching } \\
\text { - Time: } 300 \mathrm{sec}\end{array}$ & \\
\hline 20 & Wet etching of TEOS & $\begin{array}{l}\mathrm{BHF}\left(\mathrm{NH}_{4} \mathrm{~F}: \mathrm{HF}=7: 1\right) \text { : } \\
\text { VLSI-Etch mixture } \mathrm{AF}, 87.5-12.5 \\
\text { - Quick Dump Rinse }<0.1 \mu \mathrm{S} \\
\text { - Spin drying } \\
\text { Etch rate }=180 \mathrm{~nm} / \mathrm{min} \text { (for not-annealed } \\
\text { TEOS) }\end{array}$ & Time $: 2 \min$ \\
\hline 21 & $\begin{array}{l}\text { Optical microscopic } \\
\text { inspection-Lithography }\end{array}$ & Microscope & \\
\hline 22 & $\begin{array}{l}\text { Stripping of polymers in } \\
\mathrm{HNO}_{3} \text { multipurpose }\end{array}$ & $\begin{array}{l}\mathrm{HNO}_{3}(99 \%) \text { Selectipur: MERCK } \\
\text { - Beaker } 1: \mathrm{HNO}_{3}(99 \%) \\
\text { - Time : } 20 \mathrm{~min} \\
\text { - Quick Dump Rinse }<0.1 \mu \mathrm{S} \\
\text { - Spin drying }\end{array}$ & \\
\hline 23 & $\begin{array}{l}\text { Wet etching of } \mathrm{SiN}_{\mathrm{x}} \\
\left(\mathrm{Hot} \mathrm{H}_{3} \mathrm{PO}_{4}\right)\end{array}$ & $\begin{array}{l}\mathrm{H}_{3} \mathrm{PO}_{4}(100 \%): \text { MERCK VLSI } 1.00568 .2500 \\
\text { - Temperature : } 180^{\circ} \mathrm{C} \\
\text {-Quick Dump Rinse }<0.1 \mu \mathrm{S} \\
\text { - Spin drying } \\
\text { Etch rate for } \mathrm{SiN}_{\mathrm{x}} \sim 5 \mathrm{~nm} / \mathrm{min} \\
\text { Etch rate for TEOS } \sim 3.5 \mathrm{~nm} / \mathrm{min}\end{array}$ & $\begin{array}{l}\text { Check the etch rate and } \\
\text { selectivity between } \\
\text { TEOS and } \mathrm{SiN}_{\mathrm{x}} \text { each } \\
\text { time with dummy } \\
\text { wafers. }\end{array}$ \\
\hline 24 & $\begin{array}{l}\text { Optical microscopic } \\
\text { inspection }\end{array}$ & Microscope & \\
\hline 25 & Cleaning Standard & $\begin{array}{l}\mathrm{HNO}_{3}(99 \%) \text { Selectipur: MERCK } \\
\mathrm{HNO}_{3}(69 \%) \text { VLSI: MERCK } \\
\text { - Beaker 1: fumic } \mathrm{HNO}_{3}(99 \%), 5 \mathrm{~min} \\
\text { - Beaker 2: fumic } \mathrm{HNO}_{3}(99 \%), 5 \mathrm{~min} \\
\text { - Quick Dump Rinse }<0.1 \mu \mathrm{S} \\
\text { - Beaker 3: boiling }\left(95^{\circ} \mathrm{C}\right) \mathrm{HNO}_{3}(69 \%), 10 \\
\text { min } \\
\text { - Quick Dump Rinse }<0.1 \mu \mathrm{S} \\
\text { - Spin drying }\end{array}$ & \\
\hline
\end{tabular}




\begin{tabular}{|c|c|c|c|}
\hline 26 & LPCVD TEOS & $\begin{array}{l}\text { Tempress LPCVD B4 } \\
\text { Tube: B4 TEOS } \\
\text { - Bubbler: } 40^{\circ} \mathrm{C} \\
\text { - } \mathrm{N}_{2} \text { flow: } 250 \mathrm{sccm} \\
\text { - Temperature: } 700{ }^{\circ} \mathrm{C} \\
\text { - Pressure: } 150 \mathrm{~m} \text { Torr } \\
\text { - Program: TEOS } 05 \\
\text { Deposition rate: } 10.7 \mathrm{~nm} / \mathrm{min} \\
\mathrm{N}_{\mathrm{f}} \cdot 1.44\end{array}$ & $\begin{array}{l}\text { Thickness } \sim 100 \mathrm{~nm} \\
\text { Time } \sim 00: 11 \mathrm{hr}: \mathrm{min} \\
\text { Use at least } 6 \text { dummy } \\
\text { wafers }\end{array}$ \\
\hline 27 & $\begin{array}{l}\text { Ellipsometer } \\
\text { Measurement }\end{array}$ & Metrology Woollam ellipsometer & $\begin{array}{l}\text { Measure the layer } \\
\text { thickness on dummy } \\
\text { silicon wafers }\end{array}$ \\
\hline 28 & $\begin{array}{l}\text { Wafer curvature } \\
\text { measurement }\end{array}$ & $\begin{array}{l}\text { Veeco Dektak } 8 \\
\text { To measure the stress in the deposited } \operatorname{SiN}_{x} \\
\text { layer }\end{array}$ & $\begin{array}{l}\text { Measure on dummy } \\
\text { silicon wafers at } 0^{\circ}, 45^{\circ} \text {, } \\
90^{\circ} \text { and at } 135^{\circ} \text { and use } \\
\text { them in steps } 29,30 \text { and } \\
32 \mathrm{~A} \text {. }\end{array}$ \\
\hline 29 & Cleaning Standard & $\begin{array}{l}\mathrm{HNO}_{3}(99 \%) \text { Selectipur: MERCK } \\
\mathrm{HNO}_{3}(69 \%) \text { VLSI: MERCK } \\
\text { - Beaker 1: fumic } \mathrm{HNO}_{3}(99 \%), 5 \mathrm{~min} \\
\text { - Beaker 2: fumic } \mathrm{HNO}_{3}(99 \%), 5 \mathrm{~min} \\
\text { - Quick Dump Rinse <0.1 } \mu \mathrm{S} \\
\text { - Beaker 3: boiling }\left(95^{\circ} \mathrm{C}\right) \mathrm{HNO}_{3}(69 \%), 10 \\
\text { min } \\
\text { - Quick Dump Rinse <0.1 } \mu \mathrm{S} \\
\text { - Spin drying }\end{array}$ & \\
\hline 30 & $\begin{array}{l}\text { LPCVD SiN }- \text { low } \\
\text { stress }\end{array}$ & $\begin{array}{l}\text { Tempress LPCVD/HC } \\
\text { Tube: } \mathrm{G} 3 \\
\text { - } \mathrm{SiH}_{2} \mathrm{Cl}_{2} \text { flow: } 70 \mathrm{sccm} \\
\text { - } \mathrm{NH}_{3} \text { flow: } 18 \mathrm{sccm} \\
\text { - Temperature: } 830{ }^{\circ} \mathrm{C} \\
\text { - Pressure: } 200 \mathrm{~m} \text { Torr } \\
\text { - Program: SiRN01 } \\
\text { Deposition rate: } 3.9 \sim 5.3 \mathrm{~nm} / \mathrm{min} \\
\mathrm{N}_{f} \cdot 2.14\end{array}$ & $\begin{array}{l}\text { Thickness } \sim 1.0 \mu \mathrm{m} \\
\text { Time } \sim 03: 55 \mathrm{hr}: \mathrm{min} \\
\text { Use at least } 6 \text { dummy } \\
\text { wafers. (keep two slot } \\
\text { gap in the boat between } \\
\text { adjacent wafers) }\end{array}$ \\
\hline 31 & $\begin{array}{l}\text { Ellipsometer } \\
\text { Measurement }\end{array}$ & Metrology Woollam ellipsometer & $\begin{array}{l}\text { For measuring layer } \\
\text { thickness on dummy } \\
\text { silicon wafer }\end{array}$ \\
\hline 32 & $\begin{array}{l}\text { Wafer curvature } \\
\text { measurement }\end{array}$ & $\begin{array}{l}\text { Veeco Dektak } 8 \\
\text { (A) For SiN } \mathbf{N}_{\mathbf{x}} \text { layer: Use the silicon wafers } \\
\text { measured in step 28. Etch the SiN } \mathrm{N}_{\mathrm{x}} \text { on the back } \\
\text { side using step 08. And measure the final wafer } \\
\text { curvature. } \\
\text { (B) For Au+Cr layer: Use new silicon dummy } \\
\text { wafers and measure the initial wafer curvature. }\end{array}$ & $\begin{array}{l}\text { Measure the wafer } \\
\text { curvature at } 0^{\circ}, 45^{\circ}, 90^{\circ} \\
\text { and at } 135^{\circ} \text {. }\end{array}$ \\
\hline 33 & Cleaning Standard & $\begin{array}{l}\mathrm{HNO}_{3}(99 \%) \text { Selectipur: MERCK } \\
\mathrm{HNO}_{3}(69 \%) \text { VLSI: MERCK } \\
\text { - Beaker 1: fumic } \mathrm{HNO}_{3}(99 \%), 5 \mathrm{~min} \\
\text { - Beaker 2: fumic } \mathrm{HNO}_{3}(99 \%), 5 \mathrm{~min}\end{array}$ & \\
\hline
\end{tabular}




\begin{tabular}{|c|c|c|c|}
\hline & & $\begin{array}{l}\text { - Quick Dump Rinse }<0.1 \mu \mathrm{S} \\
\text { - Beaker 3: boiling }\left(95^{\circ} \mathrm{C}\right) \mathrm{HNO}_{3}(69 \%), 10 \\
\text { min } \\
\text { - Quick Dump Rinse }<0.1 \mu \mathrm{S} \\
\text { - Spin drying }\end{array}$ & \\
\hline 34 & Sputtering of $\mathrm{Cr}$ & $\begin{array}{l}\text { Sputterke } \\
\text { - Electrode temperature : Water cooled } \\
\text { electrode } \\
\text { - Power: } 100 \mathrm{~W} \\
\text { - Base Pressure: }<1 \times 10^{-6} \mathrm{mbar} \\
\text { - Sputter Pressure: }<6.6{\mathrm{X} 10^{-3} \mathrm{mbar}} \\
\text { Deposition rate }(100 \mathrm{~mm} \text { wafer }): 15 \mathrm{~nm} / \mathrm{min}\end{array}$ & Thickness: $8 \mathrm{~nm}$ \\
\hline 35 & Sputtering of $\mathrm{Au}$ & $\begin{array}{l}\text { Sputterke } \\
\text { - Electrode temperature : Water cooled } \\
\text { electrode } \\
\text { - Power: } 200 \mathrm{~W} \\
\text { - Base Pressure: }<1 \times 10^{-6} \mathrm{mbar} \\
\text { - Sputter Pressure: }<6.6{\mathrm{X} 10^{-3} \mathrm{mbar}} \\
\text { Deposition rate }(100 \mathrm{~mm} \text { wafer }): 45-50 \\
\mathrm{~nm} / \mathrm{min}\end{array}$ & Thickness: $50 \mathrm{~nm}$ \\
\hline 36 & $\begin{array}{l}\text { Wafer curvature } \\
\text { measurement }\end{array}$ & $\begin{array}{l}\text { Veeco Dektak } 8 \\
\text { Use silicon dummy wafers used in step } 32 \mathrm{~B} \text {. }\end{array}$ & $\begin{array}{l}\text { Measure at } 0^{\circ}, 45^{\circ}, 90^{\circ} \\
\text { and at } 135^{\circ} \text {. }\end{array}$ \\
\hline 37 & $\begin{array}{l}\text { Layer Thickness } \\
\text { Measurement }\end{array}$ & Veeco Dektak 8 & $\begin{array}{l}\text { Remove the scotch tape } \\
\text { from dummy wafers } \\
\text { and measure the step } \\
\text { height of } \mathrm{Au} \text { and } \mathrm{Cr} \\
\text { layers. }\end{array}$ \\
\hline 38 & $\begin{array}{l}\text { Lithography - Priming } \\
\text { (liquid) }\end{array}$ & $\begin{array}{l}\text { HexaMethylDiSilazane (HMDS) } \\
\text { - Dehydration bake }\left(120^{\circ} \mathrm{C}\right): 5 \mathrm{~min} \\
\text { - Spin program: } 5(5000 \mathrm{rpm}, 20 \mathrm{sec})\end{array}$ & \\
\hline 39 & $\begin{array}{l}\text { Lithography - Coating } \\
\text { Olin907-17 }\end{array}$ & $\begin{array}{l}\text { - Spinning acceleration: } 4000 \mathrm{rpm} / \mathrm{s} \\
\text { - Spinning speed: } 4000 \mathrm{rpm} \\
\text { - Spinning time: } 20 \mathrm{~s} \\
\text { - Prebake }\left(95^{\circ} \mathrm{C}\right): 90 \mathrm{~s}\end{array}$ & $\begin{array}{l}\text { Wait } 30 \text { min before } \\
\text { exposure to avoid } \\
\text { stiction }\end{array}$ \\
\hline 40 & $\begin{array}{l}\text { Lithography - } \\
\text { Alignment \& Exposure } \\
\text { Olin } 907-17\end{array}$ & $\begin{array}{l}\text { Electronic Vision Group } 20 \text { Mask Aligner } \\
\text { - Hg-lamp: } 12 \mathrm{~mW} / \mathrm{cm}^{2} \\
\text { - Exposure Time: } 4.1 \mathrm{sec}\end{array}$ & $\begin{array}{l}\text { Mask: for patterning } \\
\text { metal layers } \\
\text { Hard contact }\end{array}$ \\
\hline 41 & $\begin{array}{l}\text { Lithography - } \\
\text { Development Olin } \\
\text { Resist }\end{array}$ & $\begin{array}{l}\text { Developer: OPD4262 } \\
\text { - After Exposure Bake }\left(120^{\circ} \mathrm{C}\right): 60 \mathrm{sec} \\
\text { Development: } \\
\text { - Time: } 30 \mathrm{sec} \text { in Beaker } 1 \\
\text { - Time: } 15-30 \mathrm{sec} \text { in Beaker } 2 \\
\text { - Quick Dump Rinse }<0.1 \mu \mathrm{S} \\
\text { - Spin drying }\end{array}$ & \\
\hline 42 & $\begin{array}{l}\text { Optical microscopic } \\
\text { inspection-Lithography }\end{array}$ & Microscope & \\
\hline
\end{tabular}




\begin{tabular}{|c|c|c|c|}
\hline 43 & $\begin{array}{l}\text { Lithography - Postbake } \\
\text { standard }\end{array}$ & Hot plate at $120^{\circ} \mathrm{C}: 30 \mathrm{~min}$ & \\
\hline 44 & $\begin{array}{l}\text { Ozone anneal of Olin } \\
907\end{array}$ & $\begin{array}{l}\text { UV PRS-100 } \\
\text { - To improve wetting during etching } \\
\text { - Time: } 300 \mathrm{sec}\end{array}$ & \\
\hline 45 & Wet etching of $\mathrm{Au}$ & $\begin{array}{l}\text { Gold etchant: }\left(\mathrm{KI}=132 \mathrm{~g}, \mathrm{I}_{2}=18 \mathrm{~g}, \mathrm{DI}=\right. \\
1200 \mathrm{ml} \text {, in which glycerin }(600 \mathrm{ml}) \text { is added to } \\
\text { reduce the excessive undercut }) \\
\text { - Temperature }: 30^{\circ} \mathrm{C} \\
\text { - Quick Dump Rinse }<0.1 \mu \mathrm{S} \\
\text { - Spin drying } \\
\text { Etch rate }=1 \mathrm{~nm} / \mathrm{min}\end{array}$ & Time $=50 \mathrm{sec}$ \\
\hline 46 & Wet etching of $\mathrm{Cr}$ & $\begin{array}{l}\text { Chromium etch LSI selectipur : MERCK } \\
111547.2500 \\
\text { - Quick Dump Rinse }<0.1 \mu \mathrm{S} \\
\text { - Spin drying } \\
\text { Etch rate }=0.5 \mathrm{~nm} / \mathrm{min}\end{array}$ & \\
\hline 47 & $\begin{array}{l}\text { Optical microscopic } \\
\text { inspection-Lithography }\end{array}$ & Microscope & \\
\hline 48 & $\begin{array}{l}\text { Stripping of polymers in } \\
\mathrm{HNO}_{3} \text { multipurpose }\end{array}$ & $\begin{array}{l}\mathrm{HNO}_{3}(99 \%) \text { Selectipur: MERCK } \\
\text { - Beaker } 1: \mathrm{HNO}_{3}(99 \%) \\
\text { - Time : } 20 \mathrm{~min} \\
\text { - Quick Dump Rinse <0.1 } \mathrm{\mu S} \\
\text { - Spin drying }\end{array}$ & \\
\hline 49 & $\begin{array}{l}\text { Lithography - Priming } \\
\text { (liquid) }\end{array}$ & $\begin{array}{l}\text { HexaMethylDiSilazane (HMDS) } \\
\text { - Dehydration bake }\left(120^{\circ} \mathrm{C}\right): 5 \mathrm{~min} \\
\text { - Spin program: } 5(5000 \mathrm{rpm}, 20 \mathrm{sec})\end{array}$ & \\
\hline 50 & $\begin{array}{l}\text { Lithography - Coating } \\
\text { Olin907-35 }\end{array}$ & $\begin{array}{l}\text { - Spinning acceleration: } 4000 \mathrm{rpm} / \mathrm{s} \\
\text { - Spinning speed: } 4000 \mathrm{rpm} \\
\text { - Spinning time: } 20 \mathrm{~s} \\
\text { - Prebake }\left(95^{\circ} \mathrm{C}\right): 90 \mathrm{~s}\end{array}$ & $\begin{array}{l}\text { Wait } 30 \text { min before } \\
\text { exposure to avoid } \\
\text { stiction }\end{array}$ \\
\hline 51 & $\begin{array}{l}\text { Lithography - } \\
\text { Alignment \& Exposure } \\
\text { Olin } 907-35\end{array}$ & $\begin{array}{l}\text { Electronic Vision Group } 20 \text { Mask Aligner } \\
\text { - Hg-lamp: } 12 \mathrm{~mW} / \mathrm{cm}^{2} \\
\text { - Exposure Time: } 9.3 \mathrm{sec}\end{array}$ & $\begin{array}{l}\text { Mask: For patterning } \\
\text { SiN }_{x} \text { cantilever layer } \\
\text { Hard contact }\end{array}$ \\
\hline 52 & $\begin{array}{l}\text { Lithography - } \\
\text { Development Olin } \\
\text { Resist }\end{array}$ & $\begin{array}{l}\text { Developer: OPD } 4262 \\
\text { - After Exposure Bake }\left(120{ }^{\circ} \mathrm{C}\right): 60 \mathrm{sec} \\
\text { Development: } \\
\text { - Time: } 30 \mathrm{sec} \text { in Beaker } 1 \\
\text { - Time: } 15-30 \mathrm{sec} \text { in Beaker } 2 \\
\text { - Quick Dump Rinse }<0.1 \mu \mathrm{S} \\
\text { - Spin drying }\end{array}$ & $\begin{array}{l}\text { Wait at least } 30 \mathrm{~min} \\
\text { before post bake }\end{array}$ \\
\hline 53 & $\begin{array}{l}\text { Optical microscopic } \\
\text { inspection-Lithography }\end{array}$ & Microscope & \\
\hline 54 & Lithography - Postbake & Hot plate at $120^{\circ} \mathrm{C}: 30 \mathrm{~min}$ & \\
\hline
\end{tabular}




\begin{tabular}{|c|c|c|c|}
\hline & standard & & \\
\hline 55 & $\begin{array}{l}\text { Plasma etching } \mathrm{SiN}_{\mathrm{x}} \\
\text { (Etske) }\end{array}$ & $\begin{array}{l}\text { Elektrotech PF310/340 } \\
\text { Dirty chamber } \\
\text { Styros electrode } \\
\text { - Electrode temp.: } 10{ }^{\circ} \mathrm{C} \\
\text { - } \mathrm{CHF}_{3} \text { flow: } 25 \mathrm{sccm} \\
\text { - } \mathrm{O}_{2} \text { flow: } 5 \mathrm{sccm} \\
\text { - Pressure: } 10 \mathrm{mTorr} \\
\text { - Power: } 75 \mathrm{~W} \\
\text { Etchrate } \mathrm{SiN}_{\mathrm{x}}=55 \mathrm{~nm} / \mathrm{min}\left(\text { for } \mathrm{V}_{\mathrm{DC}}=-460 \mathrm{~V}\right) \\
\text { Etchrate Olin resist }=95 \mathrm{~nm} / \text { min } \\
\text { If DC-Bias }<375 \mathrm{~V} \text { apply chamber clean }\end{array}$ & $\begin{array}{l}\text { Backside } \\
\text { Time: } 25 \mathrm{~min}\end{array}$ \\
\hline 56 & $\begin{array}{l}\text { Plasma etching } \mathrm{SiN}_{\mathrm{x}} \\
\text { (Etske) }\end{array}$ & $\begin{array}{l}\text { Elektrotech PF310/340 } \\
\text { Dirty chamber } \\
\text { Styros electrode } \\
\text { - Electrode temp.: } 10{ }^{\circ} \mathrm{C} \\
\text { - } \mathrm{CHF}_{3} \text { flow: } 25 \mathrm{sccm} \\
\text { - } \mathrm{O}_{2} \text { flow: } 5 \mathrm{sccm} \\
\text { - Pressure: } 10 \mathrm{mTorr} \\
\text { - Power: } 75 \mathrm{~W} \\
\text { Etchrate } \mathrm{SiN}_{\mathrm{x}}=55 \mathrm{~nm} / \mathrm{min}\left(\text { for } \mathrm{V}_{\mathrm{DC}}=-460 \mathrm{~V}\right) \\
\text { Etchrate } \text { Olin resist }=95 \mathrm{~nm} / \mathrm{min} \\
\text { If DC-Bias }<375 \mathrm{~V} \text { apply chamber clean }\end{array}$ & $\begin{array}{l}\text { Frontside } \\
\text { Etch rate: } 55 \mathrm{~nm} / \mathrm{min} \\
\text { Check with dummy } \\
\text { wafers exact thickness } \\
\text { of deposited } \mathrm{SiN}_{\mathrm{x}} \text { layer } \\
\text { and overetch } \\
\text { considering the } \\
\text { sacrificial layer } \\
\text { thickness } \\
\text { Etch time } \sim 20 \mathrm{~min}\end{array}$ \\
\hline 57 & $\begin{array}{l}\text { Optical microscopic } \\
\text { inspection-Lithography }\end{array}$ & Microscope & \\
\hline 58 & $\begin{array}{l}\text { Stripping of Olin PR by } \\
\text { oxygen plasma }\end{array}$ & $\begin{array}{l}\text { Tepla 300E } \\
\text { Barrel Etcher }(2.45 \mathrm{GHz}) \\
\text { - } \mathrm{O}_{2} \text { flow: } 200 \mathrm{sccm}(50 \%) \\
\text { - Power: } 500 \mathrm{~W} \\
\text { - Pressure: } 1.2 \mathrm{mbar} \\
\text { - Time: } 120 \mathrm{sec} .\end{array}$ & $\begin{array}{l}\text { To remove the } \\
\text { fluorocarbon } \\
\text { contamination } \\
\text { produced in step } 56\end{array}$ \\
\hline 59 & $\begin{array}{l}\text { Stripping of polymers in } \\
\mathrm{HNO}_{3} \text { multipurpose }\end{array}$ & $\begin{array}{l}\mathrm{HNO}_{3}(99 \%) \text { Selectipur: MERCK } \\
\text { - Beaker } 1: \mathrm{HNO}_{3}(99 \%) \\
\text { - Time : } 20 \mathrm{~min} \\
\text { - Quick Dump Rinse }<0.1 \mu \mathrm{S} \\
\text { - Spin drying }\end{array}$ & \\
\hline 60 & $\begin{array}{l}\text { Optical microscopic } \\
\text { inspection-Lithography }\end{array}$ & Microscope & \\
\hline 61 & $\begin{array}{l}\text { Sacrificial layer etching } \\
\text { and freeze drying. }\end{array}$ & $\begin{array}{l}\mathrm{BHF}\left(\mathrm{NH}_{4} \mathrm{~F}: \mathrm{HF}=7: 1\right): \\
\text { VLSI-Etch mixture AF,87.5-12.5 } \\
\text { - Time }: 52 \text { min (including overetching) }\end{array}$ & $\begin{array}{l}\text { See Appendix I for a } \\
\text { detailed procedure of } \\
\text { freeze drying }\end{array}$ \\
\hline 62 & $\begin{array}{l}\text { Optical microscopic } \\
\text { inspection }\end{array}$ & Microscope & $\begin{array}{l}\text { Final inspection of the } \\
\text { devices }\end{array}$ \\
\hline
\end{tabular}




\section{Publications}

\section{Journal Papers}

- S.M.C. Abdulla, L.J. Kauppinen, M. Dijkstra, M.J. de Boer, E. Berenschot, R.M. de Ridder and G.J.M. Krijnen, "Monolithically integrated cantilevers with self-aligned tips for wavelength tuning in a photonic crystal cavity based channel-drop filter", accepted for publication in Journal of Micromechanics and Microengineering.

- L.J. Kauppinen, S.M.C. Abdulla, M. Dijkstra, M.J. de Boer, E. Berenschot, G.J.M. Krijnen, M. Pollnau and R.M. de Ridder, "Micromechanically tuned ring resonator in silicon on insulator", Optics Letters, Vol. 36, pp. 1047-1049, (2011).

- S.M.C. Abdulla, H.V. Jansen, E. Berenschot, N.R. Tas, M. Dijkstra, M.J. de Boer, Y. Zhao and G.J.M. Krijnen, "Stringer elimination methods in surface nano and micromachining", submitted.

- S.M.C. Abdulla, L.J. Kauppinen, M. Dijkstra, M.J. de Boer, E. Berenschot, R.M. de Ridder and G.J.M. Krijnen, "MEMS integrated 2-D photonic crystal slab waveguide for enhanced dispersion tuning", submitted.

- S.M.C. Abdulla, L.J. Kauppinen, M. Dijkstra, M.J. de Boer, E. Berenschot, H.V. Jansen, R.M. de Ridder and G.J.M. Krijnen, "Tuning a racetrack ring resonator by an integrated dielectric MEMS cantilever", submitted.

- S.M.C. Abdulla, H. Yagubizade and G.J.M. Krijnen, "Analysis of resonance frequency and pull-in voltages of curled micro-bimorph cantilevers", submitted.

\section{Conference Papers}

- S.M.C. Abdulla, L.J. Kauppinen, M. de Ridder and G.J.M. Krijnen, "Integrated selfaligned tips for dispersion tuning in a photonic crystal micro-cavity", 16 ${ }^{\text {th }}$ International Conference on Solid-State Sensors, Actuators and Microsystems, Beijing, China, 2011, (Poster presentation).

- L.J. Kauppinen, S.M.C. Abdulla, G.J.M. Krijnen, M. Pollnau and R.M. de Ridder, "Electromechano-optically tuned ring resonator in silicon on insulator, monolithically integrated with a microcantilever", CLEO/Europe EQEC, Munich, Germany, 2011, (Oral presentation).

- S.M.C. Abdulla, L.J. Kauppinen, M. Dijkstra, E. Berenschot, M.J. de Boer, R.M. de Ridder, and G.J.M. Krijnen, "Mechano-optical switching in a MEMS integrated photonic 
crystal slab waveguide", International Conference on IEEE-Micro Electro Mechanical Systems, Cancun, Mexico, 2011, (Oral presentation).

- S.M. Chakkalakkal Abdulla, L.J. Kauppinen, M. Dijkstra, M.J.de Boer, E. Berenschot, R.M. de Ridder and G.J.M. Krijnen, "Mechanical tuning of optical racetrack ring resonators", in Proc. Eurosensors XXIV, Linz, Austria, 2010 (Oral presentation).

- S.M. Chakkalakkal Abdulla, L.J. Kauppinen, M. Dijkstra, M.J.de Boer, E. Berenschot, R.M. de Ridder and G.J.M. Krijnen, "Fabrication of integrated bimorphs with self aligned tips for optical switching in 2-d photonic crystal waveguides", Proc. $21^{\text {st }}$ Workshop on MicroMechanics Europe, Enschede, The Netherlands, 2010, (Poster presentation).

- S.M. Chakkalakkal Abdulla, L.J. Kauppinen, M. Dijkstra, E. Berenschot, M.J. de Boer, R.M. de Ridder and G J.M. Krijnen, "Integrated MEMS cantilevers for enhanced dispersion tuning of a photonic crystal waveguide", MicroNano Conference, Enschede, The Netherlands, 2010, (Poster presentation).

- S.M. Chakkalakkal Abdulla, L.J. Kauppinen, M. Dijkstra, M.J. de Boer, R.M. de Ridder and G.J.M. Krijnen, "Micro bimorph cantilever switches for tuning integrated optical systems", Sense of Contact 12, The Netherlands, 2010 (Poster presentation).

- S.M. Chakkalakkal Abdulla, E. Berenschot, M.J.de Boer, L.J. Kauppinen, R.M. de Ridder and G.J.M. Krijnen "Optimisation study of micro cantilevers for switching of photonic band gap crystals", in International Conference on Photonics in Switching 2009, Pisa, Italy (Poster presentation).

- S.M. Chakkalakkal Abdulla, L.J. Kauppinen, E. Berenschot, M.J. de Boer, R.M. de Ridder and G.J.M. Krijnen , "Experimental investigation on the integration studies of micro bimorph cantilevers for switching of photonic band gap crystals", MicroNano Conference, Delft, The Netherlands, 2009 (Poster Presentation).

- S.M. Chakkalakkal Abdulla and G.J.M. Krijnen, "Bimorph based active joints for nanometre scale actuation", Proc. Annual Workshop on Semiconductor Advances for Future Electronics and Sensors, Veldhoven, The Netherlands, 2007 (Poster presentation).

- S.M. Chakkalakkal Abdulla and G.J.M. Krijnen, "Optimised frequency range of active joints on nanometre scale stroke", Proc. 18 ${ }^{\text {th }}$ Workshop on MicroMechanics Europe, Guimarães, Portugal, pp. 211-214, 2007 (Poster presentation).

- S.M. Chakkalakkal Abdulla, W. C. L. Hopman, and G. Krijnen, "Tuning of photonic Bandgap crystals by NEMS actuators”, ePIXnet Winterschool, Pontresina, Switzerland, 2007 (Poster presentation). 


\section{Acknowledgements}

Last four years of doctoral study in University of Twente has been a special episode in my life and I take this opportunity to express my deep gratitude to all who have supported and encouraged me during this period.

First of all, I would like to thank my supervisor Gijs for introducing me into the wonderful world of MEMS. Gijs, I am amazed on your command on interdisciplinary fields and the way you handle professional and personal life in your busy schedule. Thank you very much for being very critical in my work and for fine tuning the researcher in me. I am grateful for the nanotechnology investment program NanoNed, by the Dutch Ministry of Economic Affairs, for financing the research work reported in this thesis. I thank Miko for providing a nice working environment in the group. Henri, thank you very much for the interesting discussions we had and for teaching me the art of paper writing. I would also like to extend my thanks to Leon, Remco, Niels and Theo for the conversations we had. I owe special thanks to Erwin and Meint, "the micromachining gurus of UT', for being the technological-backbone of my research. Pino and Henk, you have always been there for me whenever I knocked your door for a help.

This acknowledgement cannot be complete without expressing my special thanks to all the TST/Mic Mecers. Ram, you have been a wonderful friend all the years.., I still remember you on my first day over here, waiting for me in Enschede railway station to pick me and my heavy luggages. Nima, thanks for being so patient in answering my endless questions, in the past four years. I thank Susan and Satie for all the uncountable helps you have done and still doing for me. I thank Robert and Harman for writing the Samenvatting of this thesis. Hadi, thanks a lot for the nice discussions we had regarding the Galerkin method. Thanks Sandeep for answering all my questions right from legalising the birth certificate. Joost, you are one of the wonderful persons I have met in this life and you are still living in our hearts forever..... Thanks to Laura, Marcel, Natalia, Narges, Doekle, Mubassira, Ganesh, Vitaly, Kees, Berker, Harman, Ahmad, Hammad, Wabe, Johan, Jeroen, Joel, Florian and Antoine for the nice conversations we had. Thanks to Dennis, Markose and Yiping for providing me a very nice company in Hogekamp.

I wholeheartedly thank all IOMS group members for their co-operation in my project. Rene, thank you very much for patiently reading my manuscripts and for providing your critical comments. I thank Hugo, Manfred, Remco and Alyona for the fruitful discussions we had. Lasse, you have been a wonderful project mate. I appreciate your sense of humour and your critical optical designs which formed the backbone of our 
devices. I gratefully acknowledge Meindert Dijkstra for assisting me in cleanroom at a critical stage of my research. Special thanks to all the MESA+ cleanroom staff members for maintaining a smooth running of the cleanroom.

I thank Kamala aunty and Bernie uncle for giving me the warmth of their affection and making me feel at home. I really enjoyed the company of malayalee friends in The Netherlands especially Selina aunty-Thuladhar uncle , Shaji-Mercy, Babu-Indu, SandeepJalaja, Rajesh-sheeba \& ammalutty, Sekhar-bindhu, Jincy, Hrudya, Rajeevan, Vidhu, Rajesh Pullat, Sumy, Koshy, Babitha and Jagadheesh. My special gratitude to Dr. Anil Peters for providing me the adequate medication for a fracture on my ankle. I also warmly remember the fun I had with my international friends Katya, Marjolijn, Amir, Hanna and Dannis.

I am indebted to my beloved parents for what I am today. They have supported me throughout my life with their prayers and unconditional love. Shemmy, your care and love has been an inevitable part of my life. I always miss and cherish the wonderful moments I spent with my nieces Navami and Nihara. Thanks to Sirajkka for the nice conversations we had. I am grateful to my beloved parents in law and bothers \& sisters-in law, Rafeeque-Seji, Resheeda, Reseena, Reji and Shafi for their love and care.

I thank my teachers for carving my personality. Especially I thank Dr. V.P Joseph Sir for the constant encouragement he has provided. I am grateful to Dr. B.R Prasad for the wonderful work I have done in his group at Indian Institute of Astrophysics. I thank my friends in IIA; Blesson, Vigeesh, Ramya, Barath, Rumpa and Maltesh for their cheerful company and Sasi for being such a wonderful brother. Thanks a lot to Manojettan and family for their constant love and care. I thank Shahin and family for coming all the way from Dubai to be part of my defence ceremony. Special thanks to Shafi Quraishy for painting my thesis cover in such an elegant way and to Rafeeque for designing its layout.

Aditya, you are the wonderful thing ever happened in my life. I forget the whole world when I look at your cheerful face. This thesis is the result of all the wonderful moments you missed mamma and mamma missed you. Musthafa, thank you for being there for me in every aspect of my life. Your support, care and love is the essence of my life....

\section{Shahina}


Image Source : www.phdcomics.com

\section{HOW DO I LOVE YOU, THESIS? \\ LET ME COUNT THE WAYS...}
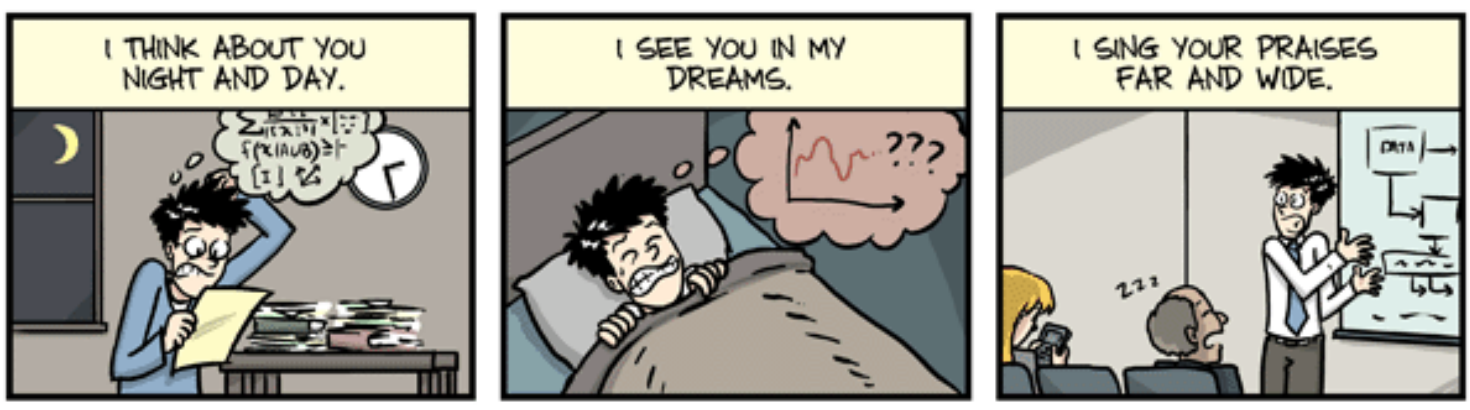

I WRITE ODES TO YOU NO ONE WILL EVER READ.
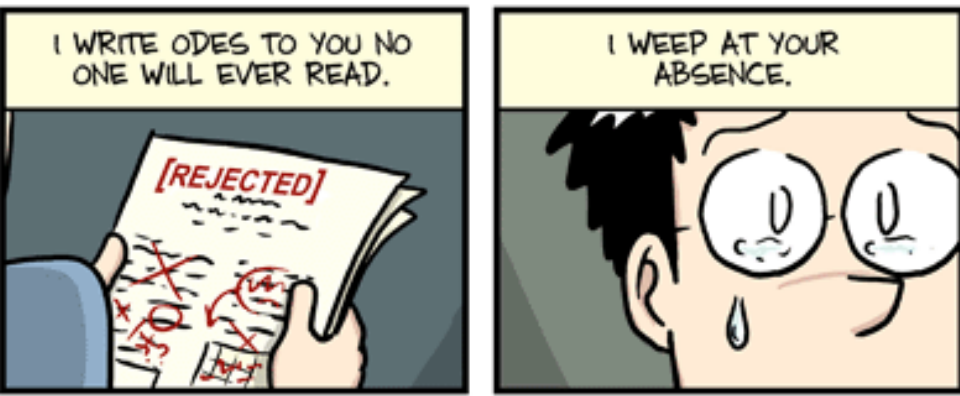

1 COUNT THE DAYSYEARS UNTL YOURE MINE.

ly published 2/11/2011

whootnote:Thanks to Alberto from Delft $U$. (again) for this comic idea! 


\section{Biography}

Shahina Mumthaz was born to C.K. Abdulla and A.K. Naphiza in Karupadanna, Kerala, India on 09th May 1981. She completed her schooling from G.H.S.S. Karupadanna, Kerala. After completing her pre-degree course from St: Joseph's College, Irinjalakkuda (Calicut University), Shahina graduated in Physics from Sacred Heart College, Chalakkudy (Calicut University) in the year 2001. In 2003, she obtained her post graduation in Physics from Christ College, Irinjalakkuda (Calicut University). Soon after this, she was offered a Junior Research Fellowship from Indian Space Research Organisation to pursue research on the contamination studies of UV optics in the UVIT/ASTROSAT project. During this period she was fascinated to the MEMS world which eventually resulted in pursuing her doctoral research at the Transducers Science and Technology group of University of Twente in The Netherlands. From April 2011, she works as a postdoctoral researcher in the Molecular Nano Fabrication group of the University of 'Twente, in the 'Nanostructured Arrays' project. 